Submitted to The Astrophysical Journal

\title{
Further Defining Spectral Type "Y" and Exploring the Low-mass End of the Field Brown Dwarf Mass Function
}

\author{
J. Davy Kirkpatrick ${ }^{a}$, Christopher R. Gelino ${ }^{a}$, Michael C. Cushing ${ }^{b}$, Gregory N. Mace ${ }^{c}$ \\ Roger L. Griffith ${ }^{a}$, Michael F. Skrutskie ${ }^{d}$, Kenneth A. Marsh ${ }^{a}$, Edward L. Wright ${ }^{c}$, Peter \\ R. Eisenhardt ${ }^{e}$, Ian S. McLean ${ }^{c}$, Amanda K. Mainzer ${ }^{e}$, Adam J. Burgasser ${ }^{f}$, C. G. \\ Tinney $^{g}$, Stephen Parker ${ }^{g}$, Graeme Salter $^{g}$
}

\begin{abstract}
We present the discovery of another seven Y dwarfs from the Wide-field Infrared Survey Explorer (WISE). Using these objects, as well as the first six WISE Y dwarf discoveries from Cushing et al., we further explore the transition between spectral types $\mathrm{T}$ and $\mathrm{Y}$. We find that the $\mathrm{T} / \mathrm{Y}$ boundary roughly coincides with the spot where the $J-H$ colors of brown dwarfs, as predicted by models, turn back to the red. Moreover, we use preliminary trigonometric parallax measurements to show that the $\mathrm{T} / \mathrm{Y}$ boundary may also correspond to the point at which the absolute $H(1.6 \mu \mathrm{m})$ and $\mathrm{W} 2(4.6 \mu \mathrm{m})$ magnitudes plummet. We use these discoveries and their preliminary distances to place them in the larger context of the Solar Neighborhood. We present a table that updates the entire stellar and substellar constinuency within 8 parsecs of the Sun, and we show that the current census has hydrogen-burning stars outnumbering brown dwarfs by roughly a factor of six. This factor will decrease with time as more brown dwarfs are identified within this volume, but unless there is a vast reservoir of cold brown dwarfs
\end{abstract}

${ }^{a}$ Infrared Processing and Analysis Center, MS 100-22, California Institute of Technology, Pasadena, CA 91125; davy@ipac.caltech.edu

${ }^{\mathrm{b}}$ Department of Physics and Astronomy, MS 111, University of Toledo, 2801 W. Bancroft St., Toledo, OH $43606-3328$

${ }^{\mathrm{c}}$ Department of Physics and Astronomy, UCLA, Los Angeles, CA 90095-1547

${ }^{\mathrm{d}}$ Department of Astronomy, University of Virginia, Charlottesville, VA, 22904

eNASA Jet Propulsion Laboratory, 4800 Oak Grove Drive, Pasadena, CA 91109

${ }^{\mathrm{f}}$ Department of Physics, University of California, San Diego, CA 92093

${ }^{g}$ Department of Astrophysics, School of Physics, University of New South Wales, NSW 2052, Australia 
invisible to WISE, the final space density of brown dwarfs is still expected to fall well below that of stars. We also use these new Y dwarf discoveries, along with newly discovered $\mathrm{T}$ dwarfs from WISE, to investigate the field substellar mass function. We find that the overall space density of late- $T$ and early-Y dwarfs matches that from simulations describing the mass function as a power law with slope $-0.5<\alpha<0.0$; however, a power-law may provide a poor fit to the observed object counts as a function of spectral type because there are tantalizing hints that the number of brown dwarfs continues to rise from late- $\mathrm{T}$ to earlyY. More detailed monitoring and characterization of these Y dwarfs, along with dedicated searches aimed at identifying more examples, are certainly required.

\section{Introduction}

The coldest field brown dwarfs hold important clues that span a variety of astronomical fields. In the field of star formation, these coldest brown dwarfs contain a historical record of the formation process at very low masses and at epochs many Gyr before the active formation regions we observe today. In the field of planetary atmospheric theory, they represent lowtemperature atmospheres that can be used as simple test cases for predictions because they lack the complications of photochemical processes produced through irradiation by a host sun. In the field of exoplanet searches, they provide interesting, nearby targets that may harbor planetary systems in their own right.

The Wide-field Infrared Survey Explorer (WISE; Wright et al. 2010) was built in part to identify these coldest brown dwarfs by using their signature methane absorption bands as a photometric diagnostic to distinguish them from myriad background sources. Specifically, the shortest wavelength WISE band, hereafter denoted W1, has a central wavelength of 3.4 $\mu \mathrm{m}$, which falls in the middle of the strong fundamental methane absorption band near 3.3

$\mu \mathrm{m}$. The second shortest WISE band, hereafter denoted W2, has a central wavelength of 4.6 $\mu \mathrm{m}$, which detects light arising from deeper, hotter layers in the brown dwarf atmosphere; at this wavelength, the atmosphere is fairly transparent to radiation, a direct analog being the 5 - $\mu \mathrm{m}$ "holes" in the atmosphere of Jupiter (Westphal 1969). As a result, the W1-W2 color can be used to identify cold brown dwarfs because that color should be very red. WISE also observes in two other bandpasses, hereafter denoted as W3 and W4, centered at 12 and 22 $\mu \mathrm{m}$, that can be used to eliminate sources with longer-wavelength flux inconsistent with that of brown dwarfs. In addition to color discrimination, WISE also benefits from observations at wavelengths where cold brown dwarfs are emitting most of their light. The all-sky nature of the survey means that the closest and brightest examples in the Solar Neigborhood will 
be imaged.

In this paper we present the discovery of another seven $\mathrm{Y}$ dwarfs - the coldest class of brown dwarfs recognized (Cushing et al. 2011) - which brings the total found with WISE to thirteen. In section 2, we discuss their selection as cold brown dwarf candidates along with their confirmation as Y dwarfs. In section 3, we discuss further the definition of spectral type $\mathrm{Y}$. In section 4, we place the $\mathrm{Y}$ dwarfs in context with other spectral classes by comparing their relative fluxes and space densities, the latter of which is also used to divine clues regarding the shape of the low-mass end of the (sub)stellar mass function. In that section, we also compare those results to several other studies that have, either directly or indirectly, probed this same mass regime.

\section{New Y Dwarf Discoveries}

\subsection{Candidate Selection}

To support the All-Sky data release in March, 2012, the WISE four-band cryogenic data were reprocessed using an improved version of the reduction pipeline, as described in the WISE All-Sky Data Release Explanatory Supplement 1 The individual W1, W2, W3, and W4 frames were rerun and atlas images re-built by stacking the individual, reprocessed frames. Source detections were made on the atlas images themselves, and source extractions were made on both the individual frames and the atlas images. Our query for cold brown dwarfs used the database of extractions from the atlas images, this database being a union of the WISE All-Sky Source Catalog and the WISE All-Sky Reject Table. This query attempts to improve upon the search we used earlier (Kirkpatrick et al. 2011) and is aimed toward identifying mid-T or later brown dwarfs for further follow-up. The search constraints are given below:

1) The W1-W2 color from profile-fit photometry is greater than 2.0 mag, where the W2 measurement is an actual detection. (This criterion guarantees that the W2 signal-to-noise ratio is greater than three.) As Figure 1 of Kirkpatrick et al. (2011) shows, this color is typical of objects of type mid-T and later.

2) The source is detected with a signal-to-noise ratio greater than three in at least eight individual W2 frames going into the coadd stack or, if detected only five, six, or seven times, is still detected at a signal-to-noise ratio greater than three in at least $40 \%$ of all frames.

\footnotetext{
${ }^{1}$ See http://wise2.ipac.caltech.edu/docs/release/allsky/.
} 
This criterion is meant to eliminate spurious, co-aligned artifacts in the coadds.

3) The source is either undetected in W3 or, if detected, has a W2-W3 color less than 3.5 mag. This criterion is meant to eliminate very red extragalactic contaminants or sources embedded in star formation regions.

4) The source is not flagged as a diffraction spike, star halo, optical ghost, or latent artifact in bands W1 and W2. This criterion is meant to remove known spurious sources. Real sources that are flagged in W1 and W2 as impacted by spikes, haloes, ghosts, or latents are, however, retained.

5) The source is not blended with another source. This criterion is meant to reduce the number of objects with poorly determined photometry.

6) The source has a reduced $\chi^{2}$ value from profile-fit photometry that lies between 0.5 and 3.0. This criterion is meant to eliminate sources that are not point-like.

7) The source has an absolute Galactic latitude greater than three degrees if its Galactic longitude falls within twenty degrees of zero. This criterion is meant to eliminate confused areas toward the Galactic Center.

With this list of sources in hand, images of the field were constructed using DSS2 BRI (epoch $\sim 1980$ s), SDSS ugriz (where available; epoch 2000), 2MASS JH $K_{s}$ (epoch 2000), and WISE four-band data (epoch $\sim 2010$ ). These image sets, an example of which is shown in Figure 1, show the source across time and across wavelength. By using these images, sources that can not possibly be cold brown dwarfs, because they are extended, spurious, or detected at wavelengths shortward of $1.0 \mu \mathrm{m}$ (unless very bright in the WISE W1 and W2 bandpasses), were eliminated from further consideration.

To select objects within $20 \mathrm{pc}$ of the Sun, we further restricted the W2 magnitude depending on the color of the source 2 : W $2 \leq 14.5$ mag for $2.0 \leq \mathrm{W} 1-\mathrm{W} 2<2.4$, W2 $\leq$ $14.8 \mathrm{mag}$ for $2.4 \leq \mathrm{W} 1-\mathrm{W} 2<2.8$, and $\mathrm{W} 2 \leq 15.2 \mathrm{mag}$ for $2.8 \leq \mathrm{W} 1-\mathrm{W} 2<2.9$. For colors redder than this $-\mathrm{W} 1-\mathrm{W} 2=2.9$ being the color of the standard T9 dwarf UGPS J072227.51-054031.2 - no W2 magnitude constraint was applied because at the time of candidate selection, the absolute magnitudes of objects with types $\geq \mathrm{T} 9$ was very poorly known. This search results in 534 candidates along with 30 re-discovered $\mathrm{T}$ dwarfs identified earlier by other surveys. Candidates have been placed on our photometry and spectroscopy campaigns, as described in the next section. To date, 189 of these 534 candidates have been

\footnotetext{
${ }^{2}$ Our team also maintains ancillary lists of candidates with bluer colors or fainter magnitudes, but those are beyond the scope of this paper.
} 
followed up via ground-based or Hubble Space Telescope imaging, all 534 are on our Spitzer Space Telescope follow-up imaging campaign, and 130 have been observed spectroscopically. This paper focuses on the new Y dwarf discoveries; for newly found T dwarfs, the reader is referred to Mace et al. (in prep.), Tinney et al. (in prep.), and Wright et al. (2012).

\subsection{Follow-up}

Cushing et al. (2011) identified the first six Y dwarfs using WISE data, and we confirm seven more here. Coordinates and photometry from the WISE All-Sky Release are given in Table 1 for all thirteen of these $\mathrm{Y}$ dwarfs. In order to facilitate future investigations, we also provide finder charts for all thirteen in Figure 2, These charts show the W1, W2, and W3 discovery images from WISE as well as a deep near-infrared $(1.2-1.6 \mu \mathrm{m})$ view at higher resolution.

Photometry of the new Y dwarfs is listed in Table 2 along with (in some cases, revised) photometry for the six Y dwarfs from Cushing et al. (2011). Six ground-based instruments and two space-based facilities - in addition to WISE, whose imaging identified these objects originally - were used for this imaging follow-up. Spectra of the new Y dwarfs were obtained in the $\sim 1.0-1.8 \mu \mathrm{m}$ region with three different instruments. Details for the spectroscopic observations are given in Table 3 ,

\subsubsection{Mt. Bigelow/2MASS}

The 2MASS camera on the 1.5m Kuiper Telescope on Mt. Bigelow, Arizona, observes simultaneously in 2MASS $J, H$, and $K_{s}$ filters (Milligan et al. 1996) using three $256 \times 256$ NICMOS3 arrays. The plate scale for all three arrays is $1^{\prime \prime} .65$ pixel $^{-1}$, resulting in a $7^{\prime} \times 7^{\prime}$ field of view. The only new Y dwarf in Table 2 whose photometry we report from the Bigelow/2MASS camera is WISE $0146+4234^{3}$. Data acquisition and reduction for this instrument have been described earlier in Kirkpatrick et al. (2011).

\footnotetext{
${ }^{3}$ Hereafter, we abbreviate the full WISE Jhhmmss.ss \pm ddmmss.s designations in the text as WISE hhmm \pm ddmm.
} 


\subsubsection{AAT/IRIS2}

The IRIS2 instrument (Tinney et al. 2004) at the 3.9m Anglo-Australian Telescope (AAT) at Siding Spring Observatory, Australia, provides wide-field imaging $(7 ! 7 \times 7 ! 7)$ using a $1024 \times 1024\left(0^{\prime \prime}\right.$.4486 pixel $\left.^{-1}\right)$ Rockwell HAWAII-1 HgCdTe infrared detector. Our observation of WISE 2220-3628 used only the $J$ filter, which is on the MKO-NIR system (Tokunaga et al. 2002). Data collection and reduction for this instrument are described in Tinney et al. (in prep.).

\subsubsection{CTIO/NEWFIRM}

The NOAO Extremely Wide Field Infrared Imager (NEWFIRM; Swaters et al. 2009) at the $4 \mathrm{~m}$ Victor M. Blanco Telescope on Cerro Tololo, Chile, uses four $2048 \times 2048 \mathrm{InSb}$ arrays arranged in a $2 \times 2$ grid. With a pixel scale of $0^{\prime \prime} 40$ pixel $^{-1}$, this grid covers a total field of view of $27 ! 6 \times 27 ! 6$. Only one of our new Y dwarfs, WISE 0734-7157, was acquired with this instrument and it was observed only at $J$ band, which is on the MKO-NIR system. Observing and reduction strategies are described in Kirkpatrick et al. (2011).

\subsubsection{SOAR/SpartanIRC}

The Spartan Infrared Camera (SpartanIRC; Loh et al. 2004) at the 4.1m Southern Astrophysics Research (SOAR) Telescope on Cerro Pachón, Chile, uses four 2048×2048-pixel HAWAII-2 arrays arranged in a $2 \times 2$ grid. The field of view can be set to cover either a $3^{\prime} \times 3^{\prime}\left(0^{\prime \prime} 043 \mathrm{pixel}^{-1}\right)$ or $5^{\prime} \times 5^{\prime}\left(0^{\prime} .073 \mathrm{pixel}^{-1}\right)$ area per array. Our only observation, of WISE 0713-2917, was done with the larger field of view in the $J$ and $H$ filters, which are on the MKO-NIR system. Observing strategy and data reductions followed the same prescription discussed in Burgasser et al. (2011).

\subsubsection{SOAR/OSIRIS}

The Ohio State Infrared Imager/Spectrometer (OSIRIS), also at SOAR, uses a $1024 \times 1024$

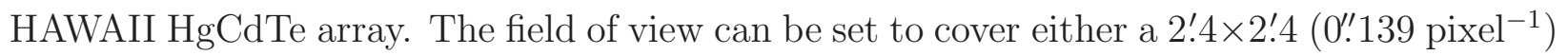
or 5!6 $\times 5 ! 6\left(0\right.$ '.331 pixel $\left.^{-1}\right)$ area. The Y dwarf WISE 0713-2917 was observed at both $J$ and $H$, and the Y dwarf WISE 2220-3628 was observed only at $H$ band. These are Barr filters; 
for $H$ band, the filter curve 4 has half-power points near 1.48 and $1.78 \mu \mathrm{m}$. Comparison of this filter curve to the filter curves illustrated in figure 4 of Bessell (2005) shows that it is very similar to the MKO-NIR $H$-band filter. Observing strategy and data reductions followed the same prescription as those of SpartanIRC (discussed in Burgasser et al. 2011).

\subsubsection{Magellan/PANIC}

Persson's Auxiliary Nasmyth Infrared Camera (PANIC; Martini et al. 2004) at the 6.5m Magellan Baade Telescope on Cerro Manqui at the Las Campanas Observatory, Chile, has a $1024 \times 1024$ HAWAII array with a plate scale of $0^{\prime \prime} .125 \mathrm{pixel}^{-1}$, resulting in a $2^{\prime} \times 2^{\prime}$ field of view. Observations of three of our new Y dwarfs - WISE 0350-5658, WISE 0359-5401, and WISE 0535-7500 - were obtained at $J$ and $H$ bands on the Carnegie (essentially MKONIR) filter system. Details regarding standard data acquisition and reduction methods can be found in Kirkpatrick et al. (2011).

\subsubsection{Spitzer/IRAC}

The Infrared Array Camera (IRAC; Fazio et al. 2004) onboard the Spitzer Space Telescope employs $256 \times 256$-pixel detector arrays to image a field of view of 5 ' $2 \times 5$ '.2 (1'.2 pixel $\left.^{-1}\right)$. IRAC was used during the warm Spitzer mission to obtain deeper photometry in its 3.6 and $4.5 \mu \mathrm{m}$ channels (hereafter, ch1 and ch2, respectively) than WISE was able to take in its W1 and W2 bands. Such observations give us more definitive colors over this wavelength regime because the WISE W1-W2 colors (Table 1) are limits only. These observations were made as part of Cycle 7 and Cycle 8 programs 70062 and 80109 (Kirkpatrick, PI) and include all thirteen of our Y dwarf discoveries (Table 2). Our standard data acquisition and reduction methodology for IRAC observations is outlined in Kirkpatrick et al. (2011).

\subsubsection{HST/WFC3}

The Wide Field Camera 3 (WFC35) onboard the Hubble Space Telescope employs a $1024 \times 1024 \mathrm{HgCdTe}$ detector with a plate scale of $0^{\prime \prime} 13$ pixel $^{-1}$ to image a field of view of

\footnotetext{
${ }^{4}$ See http://www.ctio.noao.edu/instruments/ir_instruments/osiris2soar/config/index.html.

${ }^{5}$ See http://www.stsci.edu/hst/wfc3
} 
$123^{\prime \prime} \times 126^{\prime \prime}$. It was used to obtain deep near-infrared photometry in the F140W filter (a broad bandpass encompassing most of the $J$ and $H$ bands) for seven of our Y dwarfs, as listed in Table 2. These observations were made as part of our Cycle 18 program 12330 (Kirkpatrick, PI). Photometry was measured on the drizzled images, and magnitudes were measured on the Vega system. For the brighter sources, a comparison of the photometry measured from the drizzled images to that measured from the individual, direct images showed a systematic difference that was sometimes as large as 0.20 mag. To be conservative, we have adopted $0.20 \mathrm{mag}$ as the uncertainty for all sources since this systematic difference is believed to be the dominant error term.

This same program also used the G141 grism to acquire slitless spectroscopy over the 1.1-1.7 $\mu \mathrm{m}$ range. This was done to confirm three new brown dwarf discoveries as well as to obtain better signal for a fourth, WISE $2056+1459$, in addition to those WFC3 spectra already discussed in Cushing et al. (2011) and Kirkpatrick et al. (2011). Each target was observed over a single orbit, and the integration times were selected to best fill the time available between Earth occultations. Integration times were 2212s for all objects except WISE 2056+1459, for which only 2012s could be obtained. Details of data acquisition and reduction are given in Kirkpatrick et al. (2011). Spectra of the program objects were free of contamination by other field spectra and had uncomplicated reductions except for WISE 0535-7500, whose first-order spectrum falls coincident with the second-order spectrum of a field star. The blended spectrum of this source was extracted in the same way as the others, and a correction (discussed in Section 3) was applied to mitigate the effects of the contaminating object.

\subsubsection{Keck/NIRSPEC}

The Near-Infrared Spectrometer (NIRSPEC, McLean et al. 1998, 2000) at the 10m W. M. Keck Observatory on Mauna Kea, Hawai'i, was used to confirm three of our new Y dwarfs and to obtain additional signal for a fourth, WISE 2056+1459 (Cushing et al. 2011). For spectroscopy, NIRSPEC uses a $1024 \times 1024 \mathrm{InSb}$ array. In low-resolution mode, use of the $42^{\prime \prime} \times 0^{\prime \prime} .38$ slit results in a resolving power of $\mathrm{R} \equiv \lambda / \Delta \lambda \approx 2500$. Our brown dwarf candidates were observed in either or both of the N3 and N5 configurations (see McLean et al. 2003) that cover part of the $J$-band window from 1.15 to $1.35 \mu \mathrm{m}$ and part of the $H$-band window from 1.5 to $1.8 \mu \mathrm{m}$. Standard data acquisition and reduction techniques, as described in Mace et al. (in prep.), were used. 


\subsubsection{Magellan/FIRE}

The Folded-port Infrared Echellette (FIRE; Simcoe et al. 2008, Simcoe et al. 2010) at the 6.5m Walter Baade Telescope on Cerro Manqui at the Las Campanas Observatory, Chile, uses a $2048 \times 2048$ HAWAII-2RG array. In prism mode, it covers a wavelength range from 0.8 to $2.5 \mu \mathrm{m}$ at a resolution ranging from $\mathrm{R}=500$ at $J$-band to $\mathrm{R}=300$ at $K$-band for a slit width of 0.6 . FIRE was used to confirm WISE $0734-7157$ as a Y dwar 6 . For standard data acquisition and reduction techniques for FIRE, refer to the discussion in Kirkpatrick et al. (2011).

\section{The Classification of Y Dwarfs}

\subsection{Classifying the New Discoveries}

Our new spectra, along with those from Cushing et al. (2011), are plotted in Figures 3 , 4 and 5. In Figures 3 and 4 we show all of the Y dwarfs except WISE 0535-7500 (which is plotted separately in Figure 5) and compare them to the T9.5 dwarf WISE 0148-7202 and other Y dwarfs. In Figure 5 we plot two versions of the spectrum of WISE 0535-7500. In the first, we show the contaminated spectrum as extracted by the aXe software from the Hubble Space Telescope. In the second, we plot our attempt at correcting the spectrum for the light of the contaminating object. This simplistic correction involves subtracting off a linear slope so that the mean level in the water absorption bands is zero. The resulting, quasi-corrected spectrum is sufficient to provide a crude classification despite the problems with data acquisition.

After examining these plots, several facts become apparent: (1) All seven of the new discoveries have $J$-band $(\sim 1.27 \mu \mathrm{m})$ flux peaks as narrow or narrower than the Y0 standard, WISE $1738+2732$, proposed by Cushing et al. (2011). The T9.5 dwarf, in contrast, has a wider $J$-band peak than any of these. This confirms that all are Y dwarfs, as defined by Cushing et al. (2011). (2) The spectra of WISE 1541-2250 and WISE 0350-5658 have distinctly narrower $J$-band peaks than the Y0 standard itself, meaning that both of these objects should be typed later than Y0. The plot shown in Figure 6 suggests that the $J$ band peak of WISE 0350-5658 is sufficiently narrower than WISE 1738+2732 that it should be typed a full subclass later. We therefore propose WISE 0350-5658 to be the tentative Y1 spectral standard, despite its southerly declination, until other Y1 dwarfs are identified.

\footnotetext{
${ }^{6}$ Because of the faintness of this source at $K$ band, the FIRE spectrum beyond $1.65 \mu \mathrm{m}$ is not plotted in the figures as it is very noisy.
} 
This also means that WISE 1541-2250, which has a slightly broader $J$-band peak, should be re-typed from Y0 to Y0.5. (3) The spectrum of WISE 1828+2650, though having a low signal-to-noise ratio, is still in a class by itself due to the near-equal heights of the $J$ and $H$-band $(\sim 1.58 \mu \mathrm{m})$ peaks. Because it is so different from all of the other spectra, we re-classify it to be $\geq Y 2$.

\subsection{Revisiting the Establishment of the Y Dwarf Class}

Although Cushing et al. (2011) discussed in detail the establishment of the new Y dwarf spectral class, it is worth revisiting this based on the latest discoveries. We compare our set of observational Y dwarf spectra to theoretical expectations prior to the launch of WISE. Specifically, Burrows et al. (2003) used atmospheric models covering this temperature regime to cite five possible triggers that could lead to the introduction of a new class beyond the $\mathrm{T}$ dwarfs. We investigate each of these possibilities below:

(1) The disappearance of alkali resonance lines near 450K. The resonance lines of $\mathrm{Na}$ I and K I fall at optical wavelengths, where cold brown dwarfs have little flux, but the broad wings of these lines extend very far from the line cores and are believed to have an influence on the emergent flux as longward as $1 \mu \mathrm{m}$ (Burrows \& Volobuyev 2003). As discussed in Cushing et al. (2011), the $Y$-band $(\sim 1.07 \mu \mathrm{m})$ peaks for the $Y$ dwarfs whose spectra cover that region generally appear to be as high or higher than the $J$-band peaks in units of $F_{\lambda}$. This effect is not seen in the spectra of late-T dwarfs (see, e.g., Kirkpatrick et al. 2011). A harbinger of this effect - seen as a blueward trend of $Y-J$ color in the mid- to late-T dwarf regime - was noted by Leggett et al. (2010) and Burningham et al. (2010) and ascribed to a brightening of the $Y$-band peak due to reduced absorption by the wings of the $\mathrm{K} \mathrm{I}$ as atomic potassium begins to form into $\mathrm{KCl}$ at cooler temperatures (Lodders 1999). Although indirect, this is evidence that we have pushed into the regime where these alkali lines have lost prominence. Further spectroscopic investigation at higher signal-to-noise levels is possible at $Y$-band using the G102 grism onboard HST/WFC3.

(2) Water cloud formation below 400-500K. Although long-predicted to be a possible trigger of a new spectral class at low temperatures, Burrows et al. (2003) found that the formation of these clouds has very little effect on the emergent spectra in their models. Marley (priv. comm.) also finds little effect shortward of $2 \mu \mathrm{m}$ but finds that at longer wavelengths, for objects with $\lesssim 300 \mathrm{~K}$, the effects are quite pronounced. The importance of these clouds should be re-investigated once newer atmospheric models are published for the coldest brown dwarfs. 
(3) The emergence of ammonia absorption below $2.5 \mu \mathrm{m}$. As shown in figure 5 of Kirkpatrick (2008), the theoretical spectra of Burrows et al. (2003) indicate that absorption bands of $\mathrm{NH}_{3}$, which first appear in the mid-infrared $(10.5 \mu \mathrm{m})$ near the $\mathrm{L} / \mathrm{T}$ transition (Cushing et al. 2006), finally appear at the $H$ and $K$ bands starting near $800 \mathrm{~K}$ and become prominent by 450K. As shown in figure 5 of Cushing et al. (2011), the $H$-band ammonia feature - the easier of these two to detect because the $K$-band feature falls within the telluric water band - is itself confused with overlying absorption by $\mathrm{H}_{2} \mathrm{O}$ and $\mathrm{CH}_{4}$ in the brown dwarf atmosphere. Nonetheless, signs of a possible $\mathrm{NH}_{3}$ signature were noted by Cushing et al. (2011) in the $1.53-1.58 \mu \mathrm{m}$ region of WISE $1738+2732$. This region falls in an area of extremely low flux, so exquisite signal-to-noise is needed to detect it. Unfortunately, none of the spectra of our new objects has sufficient signal to investigate this feature further. Analyzing the near-infrared spectra of these objects for the presence of $\mathrm{NH}_{3}$ bands will likely require higher resolution spectra than can be presently obtained.

(4) Collapse below $350 \mathrm{~K}$ of the optical and near-infrared fluxes, relative to those $\gtrsim 5 \mu \mathrm{m}$. Burrows et al. (2003) state that this collapse in flux would manifest itself as a reversal of the blueward trend of $J-K$ (or $J-H$ ) colors. Figure 11 in Cushing et al. (2011) shows the $J-H$ color (on the MKO-NIR system) as a function of spectral type. That figure shows that the $J-H$ color stagnates near -0.4 mag for late- $T$ dwarfs then appears to turn to the red starting at Y0, although there are some blue outliers at Y0. With our new Y dwarf discoveries, new T dwarf discoveries from Mace (et al.), additional near-infrared photometry, and revised photometric reductions of previous Y dwarf discoveries (see footnotes to Table 2), we can revisit this plot. This new photometry, shown in Figure 7 more clearly shows the reversal of the $J-H$ color. After trending to the blue from early- to mid-T and stagnating for late-T types, the $J-H$ color turns to the red starting near Y0. Later Y dwarfs, such as the Y0.5 dwarf WISE 1541-2250 ( $J-H>0.54 \mathrm{mag})$ and the $\geq$ Y2 dwarf WISE 1828+2650 $(J-H=0.72 \pm 0.42 \mathrm{mag})$ are roughly one magnitude redder than late-T dwarfs.

(5) The shift in position of the $\sim 5 \mu \mathrm{m}$ peak. As shown in figures 7 and 8 of Kirkpatrick et al. (2011), the $J-\mathrm{W} 2$ and $H-\mathrm{W} 2$ colors continue to increase from mid-T to early-Y. Figure 11 from that paper shows an indication that the Spitzer ch1-ch2 color may reverse in the Y sequence, but this was based solely on the ch1-ch2 color of WISE $1828+2650$. We can also revisit this trend using newly discovered Y dwarfs. Figure 8 shows the $J-\mathrm{W} 2, \mathrm{~F} 140 \mathrm{~W}-\mathrm{W} 2$ and $H-\mathrm{W} 2$ colors as a function of the ch1-ch2 color 7 . The ch1-ch2 color shows a broad

\footnotetext{
${ }^{7}$ Plots showing the $J-\operatorname{ch} 2, \mathrm{~F} 140 \mathrm{~W}-\mathrm{ch} 2$, and $H-\mathrm{ch} 2$ colors would look nearly identical. The W2 magnitudes listed in Table 1 are nearly identical to the ch2 magnitudes in Table 2 though the latter have errors generally two to three times smaller. However, the error in the color measurements is dominated by the $J$, F140W, or $H$ terms anyway.
} 
range (of up to a magnitude) for $\mathrm{Y} 0$ dwarfs; similar scatter is seen in the ch1-ch2 colors of late T dwarfs, which Leggett et al. (2010) attribute to gravity and/or metallicity effects. Dwarfs classified as Y1 or later are generally redder than these. It should be noted that the $\geq$ Y2 dwarf, WISE 1828+2650, indicates a turn to the blue in ch1-ch2 color at later types. (The $J-\mathrm{W} 2, \mathrm{~F} 140 \mathrm{~W}-\mathrm{W} 2$, and $H-\mathrm{W} 2$ colors, nevertheless, still tend to run redder with advancing spectral type and may serve as a proxy for temperature for these early-Y dwarfs.)

WISE 1828+2650 satisfies the last two of the possible trigger conditions discussed above. The stark contrast between its spectral morphology and that of $\mathrm{T}$ dwarfs - namely, the nearequal heights of the $J$ and $H$ peaks that is manifested as a turn to the red in the $J-H$ color - satisfies the criteria needed to define a new spectral class. Moreover, Beichman et al. have recently measured the trigonometric parallax for this object, establishing it as an instrinsically dim source (see section 4.3), and model fits by Cushing et al. (2011) suggest that its effective temperature is below 300K. With more evidence now in hand, we still reach the same conclusion as Cushing et al. (2011) that WISE 1828+2650 should be considered as the archetypal Y dwarf.

Given that the ammonia absorption bands in the near-infrared are obscured by other strong absorption bands, we lack a clear signature that defines the exact onset of the Y class. A gradual change in spectral morphology is the norm, however, at the boundary between spectral classes. For example, there is very little change in the optical morphology of a late-K dwarf and an early-M dwarf, even though mid-K and mid-M spectra are markedly different, and the same is true at the boundary between $\mathrm{M}$ and $\mathrm{L}$ classes. The $\mathrm{L} / \mathrm{T}$ transition is unusual in its sudden appearance of a major absorption species that radically alters the appearance of the spectra (over what we now know is a very small temperature range; see figure 8 of Kirkpatrick 2005). For Y dwarfs, we see a gradual change in the spectral morphology from late- $\mathrm{T}$ to early-Y, even though the spectra of a mid-T dwarf and that of WISE $1828+2650$ are very different. We have further evidence that this gradual change continues down the Y sequence, as well: the spectrum of our new Y1 dwarf WISE 0350-5658 has as an $H$-band peak relative to that of the $J$-band peak that is higher than in the Y0 standard and this presages the effect of equal-peak heights seen in WISE $1828+2650$. We therefore see no reason to deviate from the Y dwarf classification scheme presented in Cushing et al. (2011), which has so far been robust to new discoveries.

\subsection{A New Spectral Index for Early-Y Dwarfs?}

For early-Y dwarfs, we have based our classifications on the narrowness of the $J$-band peak using by-eye comparisons to our Y0 and Y1 spectral standards. Can a spectral index be 
created that distills this same information? A pre-existing index, dubbed $W_{J}$, was developed by Warren et al. (2007) to measure this narrowness of $J$-band for late-T dwarfs, but we find

that it has problems in the $\mathrm{Y}$ dwarf regime. (See figure 7 of Cushing et al. 2011 for a graphical representation of this index along with other indices measured for $\mathrm{T}$ dwarfs.) All of the $\mathrm{Y}$ dwarfs have a $\mathrm{W}_{J}$ index of $\sim 0.1$, except for WISE 1828+2650, which is very noisy. Because the $J$-band peak is so narrow for $\mathrm{Y}$ dwarfs, the numerator of the $\mathrm{W}_{J}$ index (which is the flux integrated from 1.18 to $1.23 \mu \mathrm{m}$ ) is nearing zero. A better measure of the $J$-band peak can be obtained by moving this region of integration longward so that it still falls in a region with measurable flux nearer the peak. Also, the region used in the denominator of the index (1.26 to $1.285 \mu \mathrm{m})$, which measures the flux in the peak itself, as well as the region used in the numerator (to measure the blueward wing) can be narrowed to reflect the fact that the opacity hole in the spectrum has narrowed for the Y dwarfs.

Mace et al. define a new index, called $J$-narrow, which is the ratio of the median flux over the 1.245-1.260 $\mu \mathrm{m}$ region to the median flux over $1.260-1.275 \mu \mathrm{m}$. We show the results of measuring this index on our collection of WISE-discovered $\mathrm{T}$ and $\mathrm{Y}$ dwarfs in Figure 9 , Note that for most of the $\mathrm{T}$ dwarfs, $J$-narrow has a value greater than 0.85 . $\mathrm{Y}$ dwarfs have values less than this, the Y0 standard WISE $1728+2732$ being the least narrow Y dwarf as measured by this index. (This is because our by-eye classification requires an object to have a $J$-band peak as least as narrow as that of the Y0 standard for it to be classified as a $\mathrm{Y}$ dwarf.) Whittling the wavelength region over which the index operates, though necessary, nonetheless comes at a price: high signal-to-noise spectra are required to make the index measurements robust. This is a difficult requirement to meet because Y dwarfs are notoriously hard to observe given their intrinsic faintness and the ones plotted in Figure 9 are among the closest, brightest Y dwarfs over the entire sky. Even though we present this new index for completeness, we strongly recommend that researchers classify their spectra by overplotting the late- $T$ and early- $Y$ standards rather than resorting to the use of spectral indices, since the latter technique is far more prone to error when spectra have low signalto-noise.

\section{Y Dwarfs in the Larger Context}

As theorized, old brown dwarfs in the field population were expected to be very faint given the fact that they have no sustained source of energy in their interiors. The faintness of these objects as a function of time could be modeled based on interior physics (e.g., figure 11 of Burrows et al. 1997), but the more difficult issue was predicting how frequently they might occur in nature, if at all. Using the $\mathrm{Y}$ dwarfs discovered by WISE and monitoring 
their astrometry to measure trigonometric parallaxes, we are able to demonstrate just how faint these objects are and can begin to put more solid limits on the Y dwarf space density and the shape of the mass function in the low-temperature, low-mass realm.

\subsection{Absolute Magnitudes and the H-R Diagram}

To understand the intrinsic faintness of $\mathrm{Y}$ dwarfs, we have constructed a HertzsprungRussell (H-R) diagram at $H$-band that contains a selection of main sequence stars and brown dwarfs with spectral types running the gamut from $\mathrm{O}$ through $\mathrm{Y}$. Objects were required to have high quality parallaxes and be free of complications such as being heavily reddened (which is mitigated by chosing only the most nearby examples) or belonging to a close multiple system. Our aim was to choose, if possible, at least twenty-five objects in each spectral class, spread evenly so that all integral subclasses were covered. All $H$-band magnitudes and errors were taken from 2MASS. Additional details are given below:

$O, B$, and $A$ dwarfs: Using Jim Kaler's "Stars" websit\& 8 , we selected objects identified as $\mathrm{O}$ dwarfs and retained those having trigonometric parallaxes from Hipparcos van Leeuwen 2007) that place them within 600 pc of the Sun and parallax errors less than one milliarcsecond (mas). Only ten $\mathrm{O}$ dwarfs survived the cut, the earliest being the O7 V star 15 Monocerotis. Using the same website and Hipparcos parallaxes, we selected B dwarfs lying within 500 pc and A dwarfs lying within 100 pc. Retaining only those with parallax errors under $\sim 1$ mas resulted in a sample of twenty-six B stars and forty-two A stars.

$F, G$, and $K$ dwarfs: Using SIMBAD, we selected F, G, and $\mathrm{K}$ stars whose Hipparcos parallaxes (van Leeuwen 2007) place them within 20.0, 18.2, and 13.4 pc of the Sun (i.e., parallax values exceeding 50, 55, and 75 mas), respectively. We retained those that Jim Kaler's "Stars" website confirms as having dwarf lumninosity classes. Further requiring that the Hipparcos parallaxes errors are less than $\sim 0.5$ mas resulted in a list of thirty-five $\mathrm{F}$ dwarfs, fifty-three $\mathrm{G}$ dwarfs, and twenty-eight $\mathrm{K}$ dwarfs.

$M$ dwarfs: We selected M dwarfs from the Research Constortium On Nearby Stars' (RECONS) web page that lists the hundred nearest stellar systems to the Sun 9 . Dropping objects where the 2MASS $H$-band photometry or measured spectral type is a composite of multiple components resulted in a list of seventy-two $\mathrm{M}$ dwarfs.

\footnotetext{
${ }^{8}$ See http://stars.astro.illinois.edu/sow/sowlist.html.

${ }^{9}$ The list was consulted in late December, 2011. See http://www.recons.org/.
} 
$L$ and $T$ dwarfs: We selected from the literature those $\mathrm{L}$ and $\mathrm{T}$ dwarfs having measured trigonometric parallaxes. The list is available as Table 5 of Kirkpatrick et al. (2011). Retaining only those not known to be multiple systems and having well measured 2MASS $H$-band magnitudes results in twenty $\mathrm{L}$ dwarfs and twenty-one $\mathrm{T}$ dwarfs.

$Y$ dwarfs: Due to the paucity of parallax data currently available for Y dwarfs, we retained all Y dwarfs from Marsh et al. (in prep.) and Beichman et al. (in prep.) having trigonometric parallax measurements at least five times the error. This selection yields four Y dwarfs.

The compiled list is illustrated in Figure 10, The trend of ever-dimming $H$-band magnitude as a function of later spectral type shows two well known inflection points. The first of these occurs at early-M and is a result of hydrogen associating into $\mathrm{H}_{2}$ at temperatures below 4000K (Mould 1976; Mould \& Hyland 1976). The other inflection point, at late-L to mid- $\mathrm{T}$, is a flattening or brightening of the $H$-band flux over a range of spectral types near the L/T transition (see, e.g., Looper et al. 2008, Dahn et al. 2002, Vrba et al. 2004, Knapp et al. 2004; the brightening is even more dramatic in the $J$-band). The physical cause for this brightening may be due to patchy clouds in the atmospheres of these objects (Marley et al. 2010). Below this second inflection point, the $H$-band magnitude plummets. By early- $Y$ the absolute $H$-band magnitudes are $\sim 30$ mags (or 12 orders of magnitude in flux) fainter than those of late-O dwarfs. As discussed later in this paper, the trend of absolute $H$-band flux may show another inflection point at early-Y, although more data are required to check this further.

\subsection{Y Dwarf Number Density and the 8-pc Census}

To understand the importance of Y dwarfs in the Milky Way, we consider an all-sky, volume-limited sample of the Solar Neighborhood with which to compare the frequency of Y dwarfs relative to other spectral types. Previous authors have considered different volumes as defining the immediate Solar Neighborhood. Building on earlier work 10 by Hertzsprung (1907, 1922), van de Kamp (1930, 1940, 1945, 1953, 1969, 1971) considered the sample out to $\sim 5$ pc; Kuiper (1942) considered a volume out $\sim 10$ pc; and Gliese (1956, 1969) and Gliese \& Jahreiß (1979) considered a distance limit of $\sim 20 \mathrm{pc}$, although this was extended to 25 pc in Gliese \& Jahreiß (1991). For our purposes, we will consider a distance limit of $8 \mathrm{pc}$ because this bounds a volume with a sufficient number of objects $(\sim 250)$ to provide adequate statistics across spectral classes.

\footnotetext{
10 Hertzsprung (1907) was the genesis of the H-R diagram; see Batten (1998) for more on the historical importance of this paper.
} 
Table 4 gives our update of all stars and brown dwarfs known or suspected to lie within 8 pc. This list relies heavily on previous work, most notably the list compiled by Reid \& Gizis (1997) with updates in Reid et al. (2004) and Reid (priv. comm.) and the list of the hundred nearest stars compiled by RECONS at their website. The papers by Reid et al. consider only objects with Dec $>-30^{\circ}$ and do not include several recently discovered, low-luminosity objects uncovered by surveys such as 2MASS, SDSS, DENIS, UKIDSS, and WISE; the RECONS list includes only objects with precisely determined trigonometric parallaxes and only objects within roughly 6.7 pc of the Sun. Therefore, we have combed the literature to uncover more newly discovered objects, suspects with unknown or poorly measured parallaxes, or objects near the outer limits of the 8-pc volume. Other updates in Table 4 include better characterization of previously known or newly identified multiple systems and consistent, MK-based spectral types for stars earlier than late-K (see Grav et al. 2003 and Grav et al. 2006). Further details on each column are given below.

Column 1 of Table 4 is intended to provide homage to the original discoverer or survey/mission responsible for first identifying the star as a nearby object. Exceptions are made in the case of stars with common names (e.g., Altair, Fomalhaut, and Vega), Bayer designations (e.g., $\alpha$ Cen A and B, p Eri AB), Flamsteed designations (e.g., 36 Oph ABC), or designations in old stellar catalogs (e.g., Lalande 21185, Lacaille 9352, AC+79 3888). In some cases, it is difficult to determine whether Willem Luyten or the Lowell Observatory group led by Henry Giclas was the first to discover an object because both groups were undergoing photographic proper motion surveys simultaneously. For such objects, the Luyten designation is used if the Lowell Observatory group lists one in its cross-references (Giclas et al. 1971, 1978), and the Giclas number is used if no Luyten designation is given 11 Column 2 lists alternate names.

Column 3 of Table 4 gives the running number in Gliese (1956, 1969) or Gliese \& Jahreiß (1979). SIMBAD has made it common practice to identify objects from any of these papers with a prefix of "GJ", but this was originally meant for objects only from Gliese \& Jahreiß (1979). Moreover, for new objects (identified only by "NN") in Gliese \& Jahreiß (1991), SIMBAD has created its own numbering scheme; identifiers with GJ numbers higher than GJ 2159 are solely a SIMBAD creation 12. Gliese \& Jahreiß (1991) do not provide catalog

\footnotetext{
${ }^{11}$ Luyten did not usually provide cross-references to the Giclas numbers. The Lowell group aimed to acknowledge Luyten, however, when they rediscovered one of his proper motion objects. For more details, see the interview of Dr. Henry Giclas by Robert Smith on August 12, 1987, Niels Bohr Library \& Archives, American Institute of Physics, College Park, MD, USA, http://www.aip.org/history/ohilist/5022.html.

${ }^{12}$ Likewise, we do not use LHS numbers (Luyten 1979) higher than LHS 5413 because these are also a SIMBAD creation.
} 
numbers for new objects nor are ones needed because all have published, well recognized names. Such was not necessarily the case for earlier versions of the catalog - researchers in the early 1900's, notably Robert Innes, did not always affix numbers or names to their discoveries, and readers may not have had, as we do today, easy access to discovery papers. In deference to the intent of the original publications, we give a prefix of "Gl" to objects from Gliese (1956, 1969) and "GJ" only to those objects in Gliese \& Jahreiß (1979). For other objects, the field in column 3 is left blank.

Columns 4-6 of Table 4 give the measured trigonometric parallax, its error, and the reference for the measurement. If more than one group has measured the parallax, we list the published measurement with the smallest quoted error. For cases where the error in column 5 is blank, the value in column 4 is actually a spectrophotometric estimate based on the magnitude of the object and its spectral type. Columns 7-8 give the spectral type and its reference; cases where the spectral type is estimated are annotated as such. Column 9 provides an abbreviated sexagesimal J2000 position for the object in the form hhmm \pm ddss. Column 10 gives the rank of the system in distance from the Sun, and column 11 gives the individual rank of each object; for example, Sirius A is in the fifth closest stellar system to the Sun but is tied with its companion, Sirius B, as the seventh closest star.

For reference, objects included in previous compilations of the 8-pc sample but now believed to lie beyond $8 \mathrm{pc}$ are listed in Table 5. Among these is the intriguing multiple system $\xi$ UMa (Alula Australis), in which Wright et al. (2012) have announced the discovery by WISE of a widely separated T8.5 companion.

Figure 11 graphically illustrates the 8-pc sample of Table 4 and shows that the Solar Neighborhood is dominated by $M$ dwarfs. There are twice as many $M$ dwarfs known as there are all other spectral types combined, although they comprise slightly less than half of the total stellar mass. Dwarfs of types L, T, and Y are much less common. L dwarfs are especially rare - only three examples are known within 8 pc - and represent a mix of old stars at the low-mass end of the stellar mass function and old brown dwarfs at the high-mass end of the substellar mass function 13 . The numbers of brown dwarfs then rises at later types - 22 known T dwarfs and 8 known Y dwarfs are thought to lie within this 8-pc volume.

The statistics for $\mathrm{T}$ and $\mathrm{Y}$ dwarfs are still incomplete, however. For $\mathrm{T}$ dwarfs, a few

\footnotetext{
${ }^{13}$ Using the Burgasser et al. (2004, 2007) luminosity function simulations, we find that an $\alpha=0$ power law would suggest approximately $12 \mathrm{~L}$ dwarfs within this 8-pc volume. It is difficult to imagine that previous surveys have missed three quarters of all the nearby L dwarfs, so we conclude either that the appropriate value of $\alpha$ is less than zero and/or that a power-law is a poor representation for the mass function of brown dwarfs. We return to this point in the next section
} 
late-type isolated objects may yet be found, and it is expected that $\mathrm{T}$ dwarf companions to some of the higher mass stars will continue to be uncovered. The number of $\mathrm{Y}$ dwarfs is incomplete because follow-up of Y dwarf candidates from WISE is still in its infancy and because the coldest Y dwarfs are likely beyond the detection theshold of all existing surveys. As discussed in the next section, distances to the $\mathrm{Y}$ dwarfs are only now being measured for the first time, so many of the $\mathrm{Y}$ dwarf distances in Table 4 are estimates only.

\subsection{The Low-mass End of the Field Brown Dwarf Mass Function}

We now concentrate on the low-mass tail of the field brown dwarf distribution in an updated attempt to determine the shape of the substellar mass function and its low-mass cutoff. As was done in Kirkpatrick et al. (2011), we focus on objects with spectral types of T6 or later. To sample a sufficiently large number of objects at these types, we consider distances larger than the 8-pc limit of the previous section, but we must keep in mind that samples should be reasonably complete (or completable) out to the distance limit we choose. Toward this end, we will use the depth of the WISE all-sky survey as the arbiter of the distance to which a complete census can be obtained, since it is the survey most capable of finding these coldest brown dwarfs. In Kirkpatrick et al. (2011) we found that a distance limit of 20 pc worked well for integral T6-T6.5, T7-T7.5, and T8-T8.5 bins. At T9-T9.5, it was shown that WISE may only fully sample the entire sky out to $\sim 15$ pc, so this limit was chosen for that spectral bin. The limit for $\mathrm{Y}$ dwarfs was chosen to be $10 \mathrm{pc}$, which we retain here and discuss in more detail in the discussion that follows.

Many of the objects will not have trigonometric parallax measurements, so distances must be estimated spectrophotometrically - i.e., each object's apparent magnitude will be compared to the absolute magnitudes computed for similarly typed objects whose distances have been measured. Apparent magnitudes for these cold brown dwarfs are most easily measured from the ground in the $J$ and $H$ bands and from WISE at W2. Unfortunately, due to big differences in the $J$-band filters chosen for the two most popular filter systems (2MASS and the MKO-NIR system), fluxes can vary by several tenths of a magnitude for the same object (Stephens \& Leggett 2004) because the spectral energy distributions of cold brown dwarfs are so complex at these same wavelengths. Fortunately, the $H$-band filters are very similar and as a result, $H$-band measurements show little variation between

systems (Stephens \& Leggett 2004). We will therefore use $H$ and W2 photometry to estimate distances to objects lacking parallax information.

Table 6 presents a compilation of dwarfs that have measured trigonometric parallaxes, photometry at $H$ and/or W2, and types later than T4. Trigonometric parallaxes for the Y 
dwarfs are taken from Marsh et al. (in prep.) and Beichman et al. (in prep.), although three objects from Marsh et al. - WISE 0359-5401, WISE 1541-2250, and WISE 2056+1459are omitted here because their parallax values are roughly the size of the errors themselves. Unlike the other objects in these papers, these three objects lack measurements near the maximum parallax factor (or in the case of the WISE data points, have large astrometric uncertainties) and do not yet properly constrain the size of the parallactic motion.

Data in Table 6] are used to plot the absolute magnitude versus spectral type diagrams shown in Figures 12 and 13. Figure 12 shows a sharp drop in the absolute $H$ magnitude near the $\mathrm{T} / \mathrm{Y}$ transition. The absolute $H$-band magnitude for our latest object - the $\geq \mathrm{Y} 2$ dwarf WISE $1828+2650$ - appears to indicate a stagnation or perhaps even a brightening in the $H$ flux for later $\mathrm{Y}$ dwarfs relative to ones at $\mathrm{Y} 0$ and Y1. Figure 13 shows an even more surprising result: the absolute W2 magnitude plummets at Y0 and Y1 only to rebound appreciably for WISE $1828+2650$ at $\geq$ Y 2 .

We suggest three possible scenarios to explain this observed behavior. (1) The parallax value for WISE $1828+2650$ is the only one of the Y dwarf parallax measures robust enough to trust. In this case, the plummeting absolute magnitudes and Y0 and Y1 can be discounted until longer timeline astrometric monitoring of these objects has produced results with smaller measurement errors. (2) Despite their large errors, the Y0 and Y1 points indicate a pronounced dimming of the $H$ and, more notably, W2 fluxes; the discrepant point for WISE 1828+2650 merely demonstrates that it (and not the other Y dwarfs) is the oddball, its weirdness being attributable to an unknown physical cause or misclassification due to a peculiar spectral morphology 14 (3) All trigonometric parallaxes are credible, and the observed behavior represents a real brightening of the fluxes for later $\mathrm{Y}$ dwarfs relative to ones at Y0-Y1.

It is clear that continued astrometric monitoring programs targeting $\mathrm{Y}$ dwarfs at improved precisions are critical in determining which of these scenarios is the correct one. For now, we perform weighted least-squares fits to the absolute magnitude versus spectral type diagrams with the caveat that they represent our best determinations at this time and will certainly have to be revised as future data become available. Third-order polynomials were fit to data in these diagrams both with and without WISE 1828+2650; for fits that included WISE 1828+2650, a spectral type of exactly Y2 was assumed for the object. For Figure 12, fits included the two upper limit $\mathrm{M}_{H}$ values by assuming that the limits measured in $H$-band were actual detections with errors equal to the error in the $H$-band measurement of WISE

\footnotetext{
${ }^{14}$ Note that invoking unresolved binarity for this object would account for only 0.75 mag of brightening, far below what is required to account for the W2 discrepancy.
} 
1828 (0.24 mag). Although this is an ad hoc assumption, we feel that it is better to fit (conservatively) to available data in that region rather than to drop the information entirely.

The resulting least-square fits to the data are given by the following equations:

$$
M_{H}=20.272231+1.9695993(\text { type })+0.23810003(\text { type })^{2}+0.015161356(\text { type })^{3},
$$

which includes WISE 1828+2650 (red dashed curve in Figure 12), or

$$
M_{H}=21.179419+2.9205146(\text { type })+0.53564210(\text { type })^{2}+0.043616246(\text { type })^{3},
$$

which excludes it (blue solid curve in Figure 12). Also,

$$
M_{W 2}=14.249389+0.60675032(\text { type })+0.12183371(\text { type })^{2}+0.014785180(\text { type })^{3} \text {, }
$$

which includes WISE 1828+2650 (red dashed curve in Figure 13), or

$$
M_{W 2}=15.213242+1.5891486(\text { type })+0.42455282(\text { type })^{2}+0.043434368(\text { type })^{3},
$$

which excludes it (blue solid curve in Figure 13). In each equation, the spectral type is given by type $=0$ for $\mathrm{Y} 0,1$ for $\mathrm{Y} 1,-1$ for $\mathrm{T} 9,-2$ for $\mathrm{T} 8$, etc. These relations are considered valid only over the range from $\mathrm{T} 7$ to $\mathrm{Y} 1$.

For subsequent analyses we have used only the fits that exclude WISE $1828+2650$ because these relations are needed solely to estimate distances to objects of earlier type than WISE $1828+2650$ itself. In this case, we are explicitly assuming that scenario (1) above is incorrect and that either (2) or (3) is a more credible hypothesis. If we instead discover later that this assumption is incorrect, it will have the effect of increasing the distances to the Y0 and Y1 dwarfs, which will result in a reduction of the computed space density for objects at those types.

Using these fits, we have estimated distances for each of the objects identified in our all-sky census of brown dwarfs with types $\geq$ T6 (Table 7) using their measured (or assumed) spectral types and measured $H$ and/or W2 magnitudes. These distance estimates are shown in Table 8 ,

We have checked the distance distribution of objects in each spectral type bin by performing the $V / V_{\max }$ test (Schmidt 1968), which checks the uniformity of a distribution of objects in space. The quantity $V$ is the volume of space interior to object $i$ at distance $d_{i}$, and $V_{\max }$ is the full volume of space contained within the distance limit, $d_{\max }$, of the sample. For a uniform sample, the average value, $\left\langle V / V_{\max }\right\rangle$, should be 0.5 because half of the sample should lie in the nearer half of the volume and the rest should lie in the farther half. If this number is not near 0.5 , then the sample is either non-homogeneous or incomplete. 
$V / V_{\max }$ values are given in column 8 of Table 8 for each object falling within $d_{\max }$. The average value of $V / V_{\max }$ in each integral spectral type bin is listed in Table 9 .

For our sample we find that the $\mathrm{T}$ dwarf bins have $\left\langle V / V_{\max }>\right.$ values less than 0.5. This indicates that objects near the $d_{\max }$ limit have yet to be identified, meaning that the sample is still slightly incomplete at larger distances. For Y dwarfs (except for the Y2 bin with only a single object), the $\left\langle V / V_{\max }>\right.$ values are even further below 0.5 . This indicates larger incompleteness at the furthest reaches of the volume, which is not surprising given that follow-up has so far concentrated on only the closest, brightest examples. Concentrated work on the discovery of additional Y dwarfs is still badly needed.

Table 9 lists the final number of objects in each bin along with the measured space density for each. For the mid- to late-T dwarfs, whose mapping from spectral type to absolute magnitude is reasonably well established, our two biggest sources of error are sample incompleteness and the effects of binarity (Kirkpatrick et al. 2011). For the former, we can use the $<V / V_{\max }>$ values above to estimate the extent of the incompleteness per bin. If we assume that the incompleteness lies solely in the third of the volume furthest from the Sun, then we can estimate the number of objects needed to give a value of $\left\langle V / V_{\max }\right\rangle=0.5$. If we arbitrarily place all such missing objects at the midpoint of that incomplete shell, each object would have $\left\langle V / V_{\max }\right\rangle=0.84$, which corresponds to a distance of $18.9,14.2$, and $9.4 \mathrm{pc}$, respectively, for $d_{\max }$ values of 20,15 , and $10 \mathrm{pc}$. Under these assumptions, we find that a total of 10, 12, 20, and 4 additional objects are needed to fill out the T6-6.5, T7-7.5, T8-8.5, and T9-9.5 bins, respectively.

This complete sample, however, will still suffer from the effects of unresolved binarity, the brighter magnitude of the composite system leading to a nearer distance estimate than is actually the case. This will cause a few of the nearest objects to be pushed to larger distances that still fall within $d_{\max }$, but the bigger effect will be in eliminating objects entirely from the sample that were just within the $d_{\max }$ limit before correction. Burgasser et al. (2007) summarize the results of high-resolution imaging and radial velocity surveys for very low mass objects in the field along with high-resolution imaging surveys of very low mass objects in young clusters and conclude that as many as 30\% (or more) of these objects could be binary. As a worst-case scenario, we assume that $30 \%$ of our systems are eliminated as having distances beyong $d_{\max }$ once binarity is taken into account. We then find that the sample numbers previously corrected for incompleteness should now be reduced by 14, 13, 17, and 8 objects in the T6-6.5, T7-7.5, T8-8.5, and T9-9.5 bins, respectively.

For the Y dwarf bins, the uncertain mapping from spectral type to absolute magnitude along with known incompleteness of the sample are likely the biggest contributors to the uncertainty in the space density estimates. We consider the sample incompleteness to be the 
dominant effect, and hence we believe that our measured space density estimates for the Y dwarfs should be considered lower limits.

Results are plotted in Figure 14. Also plotted are the results of luminosity function simulations by Burgasser (2007) (based on earlier work by Burgasser et al. 2004) that show the expected distribution of objects for power-law mass functions with various slopes of $\alpha$, the functional form of which is given by $d N / d M \propto M^{-\alpha}$, where $N$ is number of objects and $M$ is the mass. A nominal low-mass cutoff of $1 M_{\text {Jupiter }}$, a constant birthrate over 0.1-10 Gyr, and evolutionary models of Baraffe et al. (2003) are assumed for the simulations, as well as an overall normalization of 0.0037 objects $\mathrm{pc}^{-3}$ for stars with $0.09-0.1 \mathrm{M}_{\odot}$, based on Reid et al. (1999). We show results for $\alpha=-1,0,+1$ as well as the effect of increasing the low-mass cutoff to $5 M_{\text {Jupiter }}$ or to $10 M_{\text {Jupiter }}$ for the $\alpha=0$ model. Our measurements, shown by the purple line, are overplotted on the simulations for comparison.

It is obvious from this diagram that the density of late- $T$ dwarfs falls most closely along the $\alpha=-1.0$ model. For these results to match more closely to the $\alpha=0.0$ model would require that our current census of these objects be deficient by a factor of 2 to 3 , which does not seem plausible given that the follow-up presented in Kirkpatrick et al. (2011) and Mace et al. (in prep.) has already completed a large portion of the follow-up needed for the brightest WISE T dwarf candidates over the entire sky. Although densities as large as those predicted by an intermediate $\alpha=-0.5$ model are still possible, these brown dwarfs appear to be rarer than the $\alpha=0.0$ simulation predicts.

Turning to the $\mathrm{Y}$ dwarfs, we find that the number density climbs relative to the $\mathrm{T}$ dwarfs. This would ordinarily suggest a steeper slope $(\alpha>0.0)$, if these fits were done independently of results at higher masses. The simulations of Burgasser et al. (2004, 2007) are normalized so that the space density for low-mass stars matches observational measures, so the space densities in Figure 14 are what would be expected if the same power law applied from lowmass stars to low-mass brown dwarfs. It may simply be that the power-law approximation is not ideal at lower masses, or it may be that our space densities for $\mathrm{Y}$ dwarfs are overestimates if our trigonomeric parallaxes for the $\mathrm{Y} 0$ and $\mathrm{Y} 1$ dwarfs are systematically too large. If we use the red curves from Figures 12 and 13 (i.e., the relations including WISE 1828+2650 in the fits), we find that the number densities in the $\mathrm{Y}$ dwarf bins drop by a factor of three. At face value this would result in space densities that more closely favor a slope of $\alpha=0.0$ (but not its normalization). However, it must be kept in mind that the coldest bins in Figure 14 are based on only a handful of discoveries identified so far. Other Y dwarfs are certain to be added as other candidates objects from WISE are more fully characterized and as Y dwarf companions to higher mass objects are uncovered. Thus, we expect that these pessimistic values of the $\mathrm{Y}$ dwarf space density themselves represent only lower limits. 
Models by Burrows et al. (2003) show that a brown dwarf of mass $5 M_{\text {Jupiter }}$ takes $\sim 5$ Gyr to cool to 200K; a brown dwarf of mass $10 M_{\text {Jupiter }}$, even if formed at the same time as the Milky Way itself, has not yet had enough time to cool to 200K. Our preliminary results suggest that the low-mass cutoff for star formation is below 10 and possibly even below $5 M_{\text {Jupiter }}$ (see Figure 14). Old brown dwarfs with masses below $5 M_{\text {Jupiter }}$ fall into a temperature regime $\left(T_{\text {eff }}<200 \mathrm{~K}\right)$ that is too faint for WISE to sample a sufficient volume. As such, we may not be able to place stronger constraints on the low-mass cutoff using WISE discoveries alone.

It is possible to produce a larger number of cold $\mathrm{Y}$ dwarfs relative to $\mathrm{L}$ and $\mathrm{T}$ dwarfs - and therefore provide a better match to our results - if we drop the assumption that the star formation rate has been constant over the last 10 Gyr and instead use an exponentially declining star formation rate over time or a scenario whereby all star formation took place in the first 1 Gyr of the Milky Way's lifetime. Burgasser et al. (2004) confirm that the numbers of cold brown dwarfs is markedly higher than that of warmer brown dwarfs under both of these assumptions, but he cautions that there is little other physical evidence supporting star formation rates like these. There are other possibilities, too. The Burgasser et al. (2004) simulations do not account for relative differences in the dynamical "heating" of low-mass versus high-mass brown dwarfs over time. That is, the scale height of the lower mass objects is expected to be larger than that of the higher mass group, due to the greater influence on boosting the velocities of the lower mass brown dwarfs during encounters with other objects as they orbit the Galaxy. However, this would tend to make the low-mass brown dwarfs somewhat rarer in the Solar Neighborhood, making the mismatch between our results and the simulations even more striking.

With these caveats in mind, we compare these preliminary results to other work in the literature that has attempted to measure the substellar mass function. We select four such avenues of exploration to use as comparison: earlier wide-field surveys for field brown dwarfs, a deep search using the Spitzer Space Telescope, studies in young clusters and star formation regions, and the results of field objects indirectly detected by microlensing surveys.

\subsubsection{Comparison to Results from other Field Brown Dwarf Studies}

A few other wide-field searches have earlier attempted to determine the slope of the field mass function for objects later than spectral type T6. Metchev et al. (2008) used a cross-correlation of 2MASS with the SDSS Data Release 1 to determine a space density of $4.7_{-2.8}^{+3.1} \times 10^{-3}$ objects $\mathrm{pc}^{-3}$ over the range T6-T8. Their results favor $\alpha \approx 0$. Revlé et al. (2010) used data from the Canada-France Brown Dwarf Survey to determine space den- 
sities of $5.3_{-2.2}^{+3.1} \times 10^{-3}$ objects $\mathrm{pc}^{-3}$ for T6-T8 and $8.3_{-5.1}^{+9.0} \times 10^{-3}$ objects $\mathrm{pc}^{-3}$ for T8.5-T9. Their results favor $\alpha \lesssim 0$. Burningham et al. (2010) used data from UKIDSS to determine space densities in the range $0.30 \pm 0.20 \times 10^{-3}$ to $0.59 \pm 0.39 \times 10^{-3}$ objects pc $^{-3}$ for T6T6.5, $0.40 \pm 0.28 \times 10^{-3}$ to $0.79 \pm 0.55 \times 10^{-3}$ objects $\mathrm{pc}^{-3}$ for T7-T7.5, $0.58 \pm 0.51 \times 10^{-3}$ to $1.1 \pm 1.0 \times 10^{-3}$ objects $\mathrm{pc}^{-3}$ for T8-T8.5, and $3.1 \pm 2.9 \times 10^{-3}$ to $7.6 \pm 6.9 \times 10^{-3}$ objects pc $^{-3}$ for T9. These results favor $\alpha<0$, confirming the earlier UKIDSS analysis by Pinfield et al. (2008). Figure 15 summarizes these results for direct comparison to our results in Figure 14.

It should be noted that results from each of these studies were derived using a relatively small number of sources -4 objects with types of T6 or later for Metchev et al. (2008), 13 objects with types $\geq$ T6 for Reylé et al. (2010), and 25 objects with types $\geq$ T6 for Burningham et al. (2010). Our new WISE study uses a sample of 148 objects (Table 9) with types $\geq \mathrm{T} 6$, almost six times the number in the most data-rich of these previous surveys. It should be noted that the average distance to the Reylé et al. (2010) and Burningham et al. (2010) samples are much larger than for the sample we present in Table 7 and Table 8 , Our WISE-enabled sample is the nearest sample possible, and thus the one for which parallaxes and info on unresolved binarity will be most readily obtained, allowing for refinements of our space density numbers to unequalled precisions in the future. Nevertheless, despite the sparseness of the earlier data, those results are in general agreement with our space density results for objects earlier than T9.

\subsubsection{Comparison to Results from the Spitzer Deep, Wide-field Survey}

Eisenhardt et al. (2010) identified a sample of 14 cool brown dwarf candidates (spectral types > T7) using data from the 10-deg-square Spitzer Deep, Wide-field Survey (SDWFS). The coldest of these candidates, SDWFS J143356.62+351949.2, has colors of ch1 $-\operatorname{ch} 2=2.24 \pm 0.46$ mag and $H-\operatorname{ch} 2>5.73 \mathrm{mag}$. Using figures 11 and 14 of Kirkpatrick et al. (2011) we find that the ch1-ch2 color is typical of a T9 dwarf and the $H$-ch2 color suggests that its type is $\geq$ T8. This survey, therefore, was probing down as far as late-T but probably not to early-Y.

Eisenhardt et al. (2010) found that the assumed space density and measured color distribution of the 14 brown dwarf candidates most closely matched a Chabrier (2003) log-normal mass function, or a power-law mass function with $\alpha=1.3$, as long as the $4.5-\mu \mathrm{m}$ flux of the Burrows et al. (2003) models was adjusted downward 15 . This value of $\alpha$, however, is very high compared to the one we deduce from our measurements of field, late- $\mathrm{T}$ dwarfs in the

\footnotetext{
${ }^{15}$ Such flux suppression is expected if non-equilibrium chemistry is altering the predicted depths of the CO fundamental band at $4.7 \mu \mathrm{m}$ (Golimowski et al. 2004, Saumon et al. 2007).
} 
all-sky sample $(-0.5<\alpha<0.0)$.

New data has become available that sheds additional light on these results. SDWFS J143356.62+351949.2 was imaged with HST/WFC3 in eight exposures of 1400s duration on 2009 Dec 03, and in twelve exposures of 700s duration on 2009 Dec 16, as part of program 12044. Assuming a magnitude of $\mathrm{W} 2 \approx \mathrm{ch} 2=18.47 \mathrm{mag}$, the marginal F140W detection at $26.2 \mathrm{mag}$ is fainter than the $\sim 25 \mathrm{mag}$ expected for a T9 dwarf (see Figure 8). The F127M filter covers 1.239 to $1.308 \mu \mathrm{m}$, and from Figure 4 and Figure 6 should include at least half the light seen in the 1.193 to $1.592 \mu \mathrm{m} \mathrm{F140W}$ filter. With $<20 \%$ of the F140W bandpass, the F127M filter should thus produce a noticeably brighter magnitude if the object were a cold brown dwarf. However, the source is completely undetected in F127M, with $2 \sigma$ limit of 26.2 mag. We consider it more likely that this object is an extremely red extragalactic source. The SDWFS results, because of their depth, may have been more heavily contaminated by extragalactic objects than previously believed, leading to the higher value of $\alpha$.

\subsubsection{Comparison to the Initial Mass Function in Open Clusters and Star-Forming Regions}

Because of it proximity, relatively young age, and compactness on the sky, the Pleiades has been one of the most popular open clusters in which to hunt for brown dwarfs. Many studies have attempted to measure the slope of the substellar initial mass function in this cluster, with a sampling of results suggesting $\alpha=0-1$ (Festin 1998), $\alpha=0.6$ (Bouvier et al. 1998), and $\alpha=0.62 \pm 0.14$ (Casewell et al. 2007). Other open clusters are found to have initial mass functions that are similar to that of the Pleiades (Bastian et al. 2010). These values of $\alpha$ are slightly larger than the one we derive.

Studies of the brown dwarf population in star-formation regions is hampered by the larger distances to these areas and to complications with survey completeness, which is impacted by variable extinction across the field. However, low-mass brown dwarfs are still in a young, warm state and are easier to detect than in older clusters. Luhman (2007) compare results in Taurus, Chamaeleon I, IC 348, and the Trapezium Cluster to conclude that an initial mass function with slope $\alpha \lesssim 0$ is consistent with the ratio of the number of stars to brown dwarfs seen in those regions. Although doubts about the universality of the initial mass function persist, Andersen et al. (2008) found that this assumption is credible and, having combined observational results in seven star formation regions and open clusters in the mass range 0.03-1.0 $\mathrm{M}_{\odot}$ - including the Pleiades, the Orion Nebula Cluster, and Taurus - conclude that $\alpha<0$. These studies are in good agreement with our results using field brown dwarfs. 


\subsubsection{Comparison to the Number Density of Solivagant Objects found by Microlensing} Surveys

Sumi et al. (2011) have used the collection of observed microlensing events detected in the Microlensing Observations in Astrophysics (MOA) and Optical Gravitational Lensing Experiment (OGLE) surveys to study the random stellar and substellar populations of the Milky Way. These populations are indirectly observed as lensing sources that temporarily brighten background sources in the Galactic Bulge as they pass through the line of sight between the earth and the Bulge source. The duration of the magnification event depends upon the mass, distance, and transverse speed of the lens, and can be related back to mass using assumptions about the kinematic and space distribution of the lenses. Sumi et al. (2011) identify 474 well characterized events with which they study the field mass function of the lensing sources. They fit these results with a tripartite power-law function that, in the brown dwarf regime $\left(0.01 \leq M / M_{\odot} \leq 0.08\right)$, has an exponent of $\alpha=0.49_{-0.27}^{+0.24}$. This value of $\alpha$ is similar to that found for brown dwarfs in the Pleiades but is somewhat larger than what we find in the field.

Sumi et al. (2011) also find that another population - rogue planets - may be needed to explain results at very low masses. However, this population is detectable over the low-mass tail of the brown dwarf population only at masses below $\sim 4 M_{\text {Jupiter }}$ (Einstein radius crossing times of less than 2 days). Models from Burrows et al. (2003) predict that an old (5 Gyr) solar metallicity brown dwarf with mass of $5 M_{\text {Jupiter }}$ would have an effective temperature of only $200 \mathrm{~K}$ and would be too dim to be imaged by WISE beyond $\sim 1 \mathrm{pc}$. Given that these objects would be much rarer than low-mass brown dwarfs (see figure 2 of Sumi et al. 2011), they will not have any impact on the WISE results presented here.

\section{Conclusions}

We have presented seven new Y dwarf discoveries from WISE, bringing the total number of Y dwarf discovered by WISE to thirteen. Using these Y dwarf discoveries along with WISE $\mathrm{T}$ dwarf discoveries from Kirkpatrick et al. (2011) and Mace et al. (in prep.) and discoveries from previous searches for field brown dwarfs, we compute space densities for late- $\mathrm{T}$ and early-Y dwarfs and find that stars outnumber brown dwarfs in the Solar Neighborhood by a factor of roughly six. However, this factor is certain to shrink in the future for two reasons: (1) the census of stars within a few parsecs of the Sun is well known, whereas that for late-T and $\mathrm{Y}$ dwarfs is still incomplete, so the ratio of stars to brown dwarfs is expected to decrease in the future; (2) the field sample does not yet show any clear signature that we have probed beyond star formation's low-mass cutoff, meaning that colder, very low-mass objects likely 
exist and are too faint for even WISE to detect. This having been said, however, we expect the total number of Y dwarfs identified by WISE to be only a few dozen when follow-up is largely complete. Unless there is a vast reservoir of cold brown dwarfs invisible to WISE, the space density of stars is still expected to greatly outnumber that of brown dwarfs.

Because of the extreme faintness of these $\mathrm{Y}$ dwarfs, we have so far only been able to tease out a few hints regarding their their distances, their atmospheric details, and their variety. Future work is needed to identify more examples and to characterize more fully the physical properties of ones already recognized. The study of Y-type brown dwarfs is still in its infancy but the glimpses caught so far promise tantalizing results ahead.

\section{Acknowledgments}

We thank the referee, Sandy Leggett, for constructive comments that helped to improve the paper. This publication makes use of data products from the Wide-field Infrared Survey Explorer, which is a joint project of the University of California, Los Angeles, and the Jet Propulsion Laboratory/California Institute of Technology, funded by the National Aeronautics and Space Administration. This publication also makes use of data products from 2MASS, SDSS, and DSS. 2MASS is a joint project of the University of Massachusetts and the Infrared Processing and Analysis Center/California Institute of Technology, funded by the National Aeronautics and Space Administration and the National Science Foundation. SDSS is funded by the Alfred P. Sloan Foundation, the Participating Institutions, the National Science Foundation, the U.S. Department of Energy, the National Aeronautics and Space Administration, the Japanese Monbukagakusho, the Max Planck Society, and the Higher Education Funding Council for England. The DSS were produced at the Space Telescope Science Institute under U.S. Government grant NAG W-2166. The images of these surveys are based on photographic data obtained using the Oschin Schmidt Telescope on Palomar Mountain and the UK Schmidt Telescope.

This work is based in part on observations made with the Spitzer Space Telescope, which is operated by the Jet Propulsion Laboratory, California Institute of Technology, under a contract with NASA. Support for this work was provided by NASA through an award issued to program 70062 and 80109 by JPL/Caltech. This work is also based in part on observations made with the NASA/ESA Hubble Space Telescope, obtained at the Space Telescope Science Institute, which is operated by the Association of Universities for Research in Astronomy, Inc., under NASA contract NAS 5-26555. These observations are associated with programs \#12044 and \#12330. Support for these programs was provided by NASA through a grant from the Space Telescope Science Institute. Some of the spectroscopic data presented herein 
were obtained at the W.M. Keck Observatory, which is operated as a scientific partnership among the California Institute of Technology, the University of California and the National Aeronautics and Space Administration. The Observatory was made possible by the generous financial support of the W.M. Keck Foundation. In acknowledgement of our observing time at Keck and the IRTF, we further wish to recognize the very significant cultural role and reverence that the summit of Mauna Kea has always had within the indigenous Hawai'ian community. We are most fortunate to have the opportunity to conduct observations from this mountain.

Our research has benefitted from the M, L, and T dwarf compendium housed at DwarfArchives.org, whose server was funded by a NASA Small Research Grant, administered by the American Astronomical Society. This research has made use of the NASA/IPAC Infrared Science Archive (IRSA), which is operated by the Jet Propulsion Laboratory, California Institute of Technology, under contract with the National Aeronautics and Space Administration. We are also indebted to the SIMBAD database, operated at CDS, Strasbourg, France.

\section{REFERENCES}

Albert, L., Artigau, É., Delorme, P., et al. 2011, AJ, 141, 203

Andersen, M., Meyer, M. R., Greissl, J., \& Aversa, A. 2008, ApJ, 683, L183

Artigau, É., Doyon, R., Lafrenière, D., et al. 2006, ApJ, 651, L57

Artigau, É., Radigan, J., Folkes, S., et al. 2010, ApJ, 718, L38

Baraffe, I., Chabrier, G., Barman, T. S., Allard, F., \& Hauschildt, P. H. 2003, A\&A, 402, 701

Bastian, N., Covey, K. R., \& Meyer, M. R. 2010, ARA\&A, 48, 339

Batten, A. H. 1998, JRASC, 92, 231

Benedict, G. F., McArthur, B., Chappell, D. W., et al. 1999, AJ, 118, 1086

Bergfors, C., Brandner, W., Janson, M., et al. 2010, A\&A, 520, A54

Bessell, M. S. 2005, ARA\&A, 43, 293

Beuzit, J.-L., Ségransan, D., Forveille, T., et al. 2004, A\&A, 425, 997

Bidelman, W. P. 1985, ApJS, 59, 197 
Biller, B. A., Kasper, M., Close, L. M., Brandner, W., \& Kellner, S. 2006, ApJ, 641, L141

Boeshaar, P. C., \& Tyson, J. A. 1985, AJ, 90, 817

Bouvier, J., Stauffer, J. R., Martin, E. L., et al. 1998, A\&A, 336, 490

Boyd, M. R., Henry, T. J., Jao, W.-C., Subasavage, J. P., \& Hambly, N. C. 2011, AJ, 142, 92

Burgasser, A. J., Kirkpatrick, J. D., Brown, M. E., et al. 1999, ApJ, 522, L65

Burgasser, A. J., Wilson, J. C., Kirkpatrick, J. D., et al. 2000, AJ, 120, 1100

Burgasser, A. J., Kirkpatrick, J. D., Cutri, R. M., et al. 2000, ApJ, 531, L57

Burgasser, A. J., Kirkpatrick, J. D., Brown, M. E., et al. 2002, ApJ, 564, 421

Burgasser, A. J., McElwain, M. W., \& Kirkpatrick, J. D. 2003, AJ, 126, 2487

Burgasser, A. J., Kirkpatrick, J. D., Reid, I. N., et al. 2003, ApJ, 586, 512

Burgasser, A. J., Kirkpatrick, J. D., McElwain, M. W., et al. 2003, AJ, 125, 850

Burgasser, A. J., McElwain, M. W., Kirkpatrick, J. D., et al. 2004, AJ, 127, 2856

Burgasser, A. J., Geballe, T. R., Leggett, S. K., Kirkpatrick, J. D., \& Golimowski, D. A. 2006, ApJ, 637, 1067

Burgasser, A. J., Kirkpatrick, J. D., Cruz, K. L., et al. 2006, ApJS, 166, 585

Burgasser, A. J., Reid, I. N., Siegler, N., et al. 2007, Protostars and Planets V, 427

Burgasser, A. J. 2007, ApJ, 659, 655

Burgasser, A. J., Tinney, C. G., Cushing, M. C., et al. 2008, ApJ, 689, L53

Burgasser, A. J., Looper, D., \& Rayner, J. T. 2010, AJ, 139, 2448

Burgasser, A. J., Cushing, M. C., Kirkpatrick, J. D., et al. 2011, ApJ, 735, 116

Burgasser, A. J., Gelino, C. R., Cushing, M. C., \& Kirkpatrick, J. D. 2012, ApJ, 745, 26

Burgess, A. S. M., Moraux, E., Bouvier, J., et al. 2009, A\&A, 508, 823

Burningham, B., Pinfield, D. J., Leggett, S. K., et al. 2008, MNRAS, 391, 320

Burningham, B., Pinfield, D. J., Leggett, S. K., et al. 2009, MNRAS, 395, 1237 
Burningham, B., Pinfield, D. J., Lucas, P. W., et al. 2010, MNRAS, 406, 1885

Burrows, A., Sudarsky, D., \& Lunine, J. I. 2003, ApJ, 596, 587

Burrows, A., \& Volobuyev, M. 2003, ApJ, 583, 985

Burrows, A., Marley, M., Hubbard, W. B., et al. 1997, ApJ, 491, 856

Casewell, S. L., Dobbie, P. D., Hodgkin, S. T., et al. 2007, MNRAS, 378, 1131

Chabrier, G. 2001, ApJ, 554, 1274

Chabrier, G. 2003, PASP, 115, 763

Chiu, K., Fan, X., Leggett, S. K., et al. 2006, AJ, 131, 2722

Chiu, K., Liu, M. C., Jiang, L., et al. 2008, MNRAS, 385, L53

Costa, E., Méndez, R. A., Jao, W.-C., et al. 2005, AJ, 130, 337

Costa, E., Méndez, R. A., Jao, W.-C., et al. 2006, AJ, 132, 1234

Cuby, J. G., Saracco, P., Moorwood, A. F. M., et al. 1999, A\&A, 349, L41

Cushing, M. C., Kirkpatrick, J. D., Gelino, C. R., et al. 2011, ApJ, 743, 50

Cushing, M. C., Roellig, T. L., Marley, M. S., et al. 2006, ApJ, 648, 614

Daemgen, S., Siegler, N., Reid, I. N., \& Close, L. M. 2007, ApJ, 654, 558

Dahn, C. C., Harris, H. C., Vrba, F. J., et al. 2002, AJ, 124, 1170

Deacon, N. R., Liu, M. C., Magnier, E. A., et al. 2011, arXiv:1109.6319

Delfosse, X., Forveille, T., Udry, S., et al. 1999, A\&A, 350, L39

Delfosse, X., Forveille, T., Beuzit, J.-L., et al. 1999, A\&A, 344, 897

Delorme, P., Delfosse, X., Albert, L., et al. 2008, A\&A, 482, 961

Delorme, P., Albert, L., Forveille, T., et al. 2010, A\&A, 518, A39

Drummond, J. D., Christou, J. C., \& Fugate, R. Q. 1995, ApJ, 450, 380

Ducourant, C., Dauphole, B., Rapaport, M., Colin, J., \& Geffert, M. 1998, A\&A, 333, 882

Dupuy, T. J., \& Liu, M. C. 2012, arXiv:1201.2465 
Eisenhardt, P. R. M., Griffith, R. L., Stern, D., et al. 2010, AJ, 139, 2455

Faherty, J. K., Burgasser, A. J., West, A. A., et al. 2010, AJ, 139, 176

Fazio, G. G., et al. 2004, ApJS, 154, 10

Festin, L. 1998, A\&A, 333, 497

Forveille, T., Beuzit, J.-L., Delfosse, X., et al. 1999, A\&A, 351, 619

Garrison, R. F. 1994, The MK Process at 50 Years: A Powerful Tool for Astrophysical Insight, 60,3

Gatewood, G., \& Coban, L. 2009, AJ, 137, 402

Geballe, T. R., Knapp, G. R., Leggett, S. K., et al. 2002, ApJ, 564, 466

Geißler, K., Metchev, S., Kirkpatrick, J. D., Berriman, G. B., \& Looper, D. 2011, ApJ, 732, 56

Gelino, C. R., Kirkpatrick, J. D., Cushing, M. C., et al. 2011, AJ, 142, 57

Giclas, H. L., Burnham, R., \& Thomas, N. G. 1971, Flagstaff, Arizona: Lowell Observatory, 1971 ,

Giclas, H. L., Burnham, R., Jr., \& Thomas, N. G. 1978, Lowell Observatory Bulletin, 8, 89

Golimowski, D. A., Leggett, S. K., Marley, M. S., et al. 2004, AJ, 127, 3516

Gliese, W. 1956, ZAp, 39, 1

Gliese, W. 1969, Veroeffentlichungen des Astronomischen Rechen-Instituts Heidelberg, 22, 1

Gliese, W., \& Jahreiß, H. 1979, A\&AS, 38, 423

Gliese, W., \& Jahreiß, H. 1991, On: The Astronomical Data Center CD-ROM: Selected Astronomical Catalogs, Vol. I; L.E. Brotzmann, S.E. Gesser (eds.), NASA/Astronomical Data Center, Goddard Space Flight Center, Greenbelt, MD,

Goldman, B., Marsat, S., Henning, T., Clemens, C., \& Greiner, J. 2010, MNRAS, 405, 1140

Golimowski, D. A., Henry, T. J., Krist, J. E., et al. 2000, AJ, 120, 2082

Gray, R. O., Napier, M. G., \& Winkler, L. I. 2001, AJ, 121, 214 
Gray, R. O., Corbally, C. J., Garrison, R. F., McFadden, M. T., \& Robinson, P. E. 2003, AJ, 126, 2048

Gray, R. O., Corbally, C. J., Garrison, R. F., et al. 2006, AJ, 132, 161

Griffin, R. F. 1998, The Observatory, 118, 273

Hawley, S. L., Gizis, J. E., \& Reid, I. N. 1996, AJ, 112, 2799

Hawley, S. L., Gizis, J. E., \& Reid, N. I. 1997, AJ, 113, 1458

Heintz, W. D. 1981, ApJS, 46, 247

Henry, T. J., Johnson, D. S., McCarthy, D. W., Jr., \& Kirkpatrick, J. D. 1992, A\&A, 254, 116

Henry, T. J., \& McCarthy, D. W., Jr. 1993, AJ, 106, 773

Henry, T. J., Kirkpatrick, J. D., \& Simons, D. A. 1994, AJ, 108, 1437

Henry, T. J., Walkowicz, L. M., Barto, T. C., \& Golimowski, D. A. 2002, AJ, 123, 2002

Henry, T. J., Subasavage, J. P., Brown, M. A., et al. 2004, AJ, 128, 2460

Henry, T. J., Jao, W.-C., Subasavage, J. P., et al. 2006, AJ, 132, 2360

Herbst, W., \& Layden, A. C. 1987, AJ, 94, 150

Hershey, J. L., \& Taff, L. G. 1998, AJ, 116, 1440

Hertzsprung, E. 1907, Zeitschrift für Wissenschaftliche Photographie, Photophysik und Photochemie, 5, 86.

Hertzsprung, E. 1922, Bull. Astron. Inst. Netherlands, 1, 21

Irwin, A. W., Yang, S. L. S., \& Walker, G. A. H. 1996, PASP, 108, 580

Jao, W.-C., Henry, T. J., Subasavage, J. P., et al. 2005, AJ, 129, 1954

Jenkins, L. F. 1952, [New Haven, Yale University Observatory] 1952.,

Jenkins, J. S., Ramsey, L. W., Jones, H. R. A., et al. 2009, ApJ, 704, 975

Karataş, Y., Bilir, S., Eker, Z., \& Demircan, O. 2004, MNRAS, 349, 1069

Kasper, M., Biller, B. A., Burrows, A., et al. 2007, A\&A, 471, 655 
Keenan, P. C., \& McNeil, R. C. 1989, ApJS, 71, 245

Khrutskaya, E. V., Izmailov, I. S., \& Khovrichev, M. Y. 2010, Astronomy Letters, 36, 576

Kirkpatrick, J. D., Henry, T. J., \& McCarthy, D. W., Jr. 1991, ApJS, 77, 417

Kirkpatrick, J. D. 1992, Ph.D. Thesis,

Kirkpatrick, J. D., \& McCarthy, D. W., Jr. 1994, AJ, 107, 333

Kirkpatrick, J. D., Henry, T. J., \& Simons, D. A. 1995, AJ, 109, 797

Kirkpatrick, J. D., Reid, I. N., Liebert, J., et al. 1999, ApJ, 519, 802

Kirkpatrick, J. D., Reid, I. N., Liebert, J., et al. 2000, AJ, 120, 447

Kirkpatrick, J. D. 2005, ARA\&A, 43, 195

Kirkpatrick, J. D., Cruz, K. L., Barman, T. S., et al. 2008, ApJ, 689, 1295

Kirkpatrick, J. D. 2008, 14th Cambridge Workshop on Cool Stars, Stellar Systems, and the Sun, 384, 85

Kirkpatrick, J. D., Looper, D. L., Burgasser, A. J., et al. 2010, ApJS, 190, 100

Kirkpatrick, J. D., Cushing, M. C., Gelino, C. R., et al. 2011, ApJS, 197, 19

Knapp, G. R., Leggett, S. K., Fan, X., et al. 2004, AJ, 127, 3553

Koester, D., Voss, B., Napiwotzki, R., et al. 2009, A\&A, 505, 441

Kuiper, G. P. 1942, ApJ, 95, 201

Kuiper, V. G. P. 1943, ApJ, 97, 275

Leggett, S. K., Burningham, B., Saumon, D., et al. 2010, ApJ, 710, 1627

Leggett, S. K., Toomey, D. W., Geballe, T. R., \& Brown, R. H. 1999, ApJ, 517, L139

Liu, M. C., Delorme, P., Dupuy, T. J., et al. 2011, ApJ, 740, 108

Liu, M. C., Deacon, N. R., Magnier, E. A., et al. 2011, ApJ, 740, L32

Liu, M. C., Dupuy, T. J., \& Leggett, S. K. 2010, ApJ, 722, 311

Liu, M. C., Leggett, S. K., Golimowski, D. A., et al. 2006, ApJ, 647, 1393 
Lodders, K. 1999, ApJ, 519, 793

Lodieu, N., Pinfield, D. J., Leggett, S. K., et al. 2007, MNRAS, 379, 1423

Lodieu, N., Burningham, B., Hambly, N. C., \& Pinfield, D. J. 2009, MNRAS, 397, 258

Lodieu, N., Dobbie, P. D., Deacon, N. R., Venemans, B. P., \& Durant, M. 2009, MNRAS, 395,1631

Loh, E. D., Biel, J. D., Chen, J.-J., Davis, M., Laporte, R., \& Loh, O. Y. 2004, Proc. SPIE, 5492,1644

Looper, D. L., Kirkpatrick, J. D., \& Burgasser, A. J. 2007, AJ, 134, 1162

Looper, D. L., Gelino, C. R., Burgasser, A. J., \& Kirkpatrick, J. D. 2008, ApJ, 685, 1183

Lucas, P. W., Tinney, C. G., Burningham, B., et al. 2010, MNRAS, 408, L56

Luhman, K. L., Patten, B. M., Marengo, M., et al. 2007, ApJ, 654, 570

Luhman, K. L. 2007, ApJS, 173, 104

Luhman, K. L., Burgasser, A. J., \& Bochanski, J. J. 2011, ApJ, 730, L9

Luhman, K. L., Burgasser, A. J., Labbé, I., et al. 2012, ApJ, 744, 135

Luyten, W. J. 1979, Minneapolis: University of Minnesota, 1979, 2nd ed.,

Mainzer, A., Cushing, M. C., Skrutskie, M., et al. 2011, ApJ, 726, 30

Marley, M. S., Saumon, D., \& Goldblatt, C. 2010, ApJ, 723, L117

Marocco, F., Smart, R. L., Jones, H. R. A., et al. 2010, A\&A, 524, A38

Martini, P., Persson, S. E., Murphy, D. C., Birk, C., Shectman, S. A., Gunnels, S. M., \& Koch, E. 2004, Proc. SPIE, 5492, 1653

Mason, B. D., McAlister, H. A., Hartkopf, W. I., \& Shara, M. M. 1995, AJ, 109, 332

Masters, D., McCarthy, P., Burgasser, A. J., et al. 2012, arXiv:1204.6320

Matthews, K., Nakajima, T., Kulkarni, S. R., \& Oppenheimer, B. R. 1996, AJ, 112, 1678

McCarthy, D. W., Jr., Henry, T. J., Fleming, T. A., et al. 1988, ApJ, 333, 943

McCaughrean, M. J., Close, L. M., Scholz, R.-D., et al. 2004, A\&A, 413, 1029 
McCook, G. P., \& Sion, E. M. 2006, VizieR Online Data Catalog, 3235, 0

McLean, I. S., et al. 1998, Proc. SPIE, 3354, 566

McLean, I. S., Graham, J. R., Becklin, E. E., Figer, D. F., Larkin, J. E., Levenson, N. A., \& Teplitz, H. I. 2000, Proc. SPIE, 4008, 1048

McLean, I. S., McGovern, M. R., Burgasser, A. J., Kirkpatrick, J. D., Prato, L., \& Kim, S. S. 2003, ApJ, 596, 561

McLeod, B. A., Fabricant, D., Geary, J., et al. 2004, Proc. SPIE, 5492, 1306

Metchev, S. A., Kirkpatrick, J. D., Berriman, G. B., \& Looper, D. 2008, ApJ, 676, 1281

Milligan, S., Cranton, B. W., \& Skrutskie, M. F. 1996, Proc. SPIE, 2863, 2

Montagnier, G., Ségransan, D., Beuzit, J.-L., et al. 2006, A\&A, 460, L19

Mould, J. R. 1976, A\&A, 48, 443

Mould, J. R., \& Hyland, A. R. 1976, ApJ, 208, 399

Mugrauer, M., Seifahrt, A., Neuhäuser, R., \& Mazeh, T. 2006, MNRAS, 373, L31

Murray, D. N., Burningham, B., Jones, H. R. A., et al. 2011, MNRAS, 414, 575

Nakajima, T., Oppenheimer, B. R., Kulkarni, S. R., et al. 1995, Nature, 378, 463

Perryman, M. A. C., Lindegren, L., Kovalevsky, J., et al. 1997, A\&A, 323, L49

Pinfield, D. J., Burningham, B., Tamura, M., et al. 2008, MNRAS, 390, 304

Reid, I. N., Hawley, S. L., \& Gizis, J. E. 1995, AJ, 110, 1838

Reid, I. N., \& Gizis, J. E. 1997, AJ, 113, 2246

Reid, I. N., Kirkpatrick, J. D., Liebert, J., et al. 1999, ApJ, 521, 613

Reid, I. N., \& Cruz, K. L. 2002, AJ, 123, 2806

Reid, I. N., Kilkenny, D., \& Cruz, K. L. 2002, AJ, 123, 2822

Reid, I. N., Cruz, K. L., Laurie, S. P., et al. 2003, AJ, 125, 354

Reid, I. N., Cruz, K. L., Allen, P., et al. 2004, AJ, 128, 463

Reid, I. N., Cruz, K. L., \& Allen, P. R. 2007, AJ, 133, 2825 
Reiners, A., Seifahrt, A., Käufl, H. U., Siebenmorgen, R., \& Smette, A. 2007, A\&A, 471, L5

Reylé, C., Delorme, P., Willott, C. J., et al. 2010, A\&A, 522, A112

Riaz, B., Gizis, J. E., \& Harvin, J. 2006, AJ, 132, 866

Riedel, A. R., Murphy, S. J., Henry, T. J., et al. 2011, AJ, 142, 104

Rodgers, A. W., \& Eggen, O. J. 1974, PASP, 86, 742

Saumon, D., Marley, M. S., Leggett, S. K., et al. 2007, ApJ, 656, 1136

Schilbach, E., Röser, S., \& Scholz, R.-D. 2009, A\&A, 493, L27

Schmidt, M. 1968, ApJ, 151, 393

Scholz, R.-D., McCaughrean, M. J., Lodieu, N., \& Kuhlbrodt, B. 2003, A\&A, 398, L29

Scholz, R.-D., Lo Curto, G., Méndez, R. A., et al. 2005, A\&A, 439, 1127

Scholz, R.-D., Meusinger, H., \& Jahreiß, H. 2005, A\&A, 442, 211

Scholz, R.-D. 2010, A\&A, 510, L8

Scholz, R.-D. 2010, A\&A, 515, A92

Scholz, R.-D., Bihain, G., Schnurr, O., \& Storm, J. 2011, A\&A, 532, L5

Ségransan, D., Delfosse, X., Forveille, T., et al. 2000, A\&A, 364, 665

Seifahrt, A., Röll, T., Neuhäuser, R., et al. 2008, A\&A, 484, 429

Shkolnik, E., Liu, M. C., \& Reid, I. N. 2009, ApJ, 699, 649

Shkolnik, E. L., Hebb, L., Liu, M. C., Reid, I. N., \& Collier Cameron, A. 2010, ApJ, 716, 1522

Simcoe, R. A., et al. 2010, Proc. SPIE, 7735,

Simcoe, R. A., et al. 2008, Proc. SPIE, 7014,

Skrutskie, M. F., Cutri, R. M., Stiening, R., et al. 2006, AJ, 131, 1163

Stephens, D. C., \& Leggett, S. K. 2004, PASP, 116, 9

Strauss, M. A., Fan, X., Gunn, J. E., et al. 1999, ApJ, 522, L61 
Stumpf, M. B., Brandner, W., Joergens, V., et al. 2010, ApJ, 724, 1

Subasavage, J. P., Jao, W.-C., Henry, T. J., et al. 2009, AJ, 137, 4547

Sumi, T., Kamiya, K., Bennett, D. P., et al. 2011, Nature, 473, 349

Swaters, R. A., Valdes, F., \& Dickinson, M. E. 2009, Astronomical Data Analysis Software and Systems XVIII, 411, 506

Tinney, C. G. 1996, MNRAS, 281, 644

Tinney, C. G., Burgasser, A. J., \& Kirkpatrick, J. D. 2003, AJ, 126, 975

Tinney, C. G., Ryder, S. D., Ellis, S. C., et al. 2004, Proc. SPIE, 5492, 998

Tinney, C. G., Burgasser, A. J., Kirkpatrick, J. D., \& McElwain, M. W. 2005, AJ, 130, 2326

Tokunaga, A. T., Simons, D. A., \& Vacca, W. D. 2002, PASP, 114, 180

Tomkin, J., \& Pettersen, B. R. 1986, AJ, 92, 1424

Torres, G., Henry, T. J., Franz, O. G., \& Wasserman, L. H. 1999, AJ, 117, 562

Tsvetanov, Z. I., Golimowski, D. A., Zheng, W., et al. 2000, ApJ, 531, L61

van Altena, W. F., Lee, J. T., \& Hoffleit, E. D. 1995, New Haven, CT: Yale University Observatory, —c1995, 4th ed., completely revised and enlarged,

van de Kamp, P. 1930, Popular Astronomy, 38, 17

van de Kamp, P. 1940, Popular Astronomy, 48, 297

van de Kamp, P. 1945, PASP, 57, 34

van de Kamp, P. 1953, PASP, 65, 73

van de Kamp, P. 1969, PASP, 81, 5

van de Kamp, P. 1971, ARA\&A, 9, 103

van Leeuwen, F. 2007, A\&A, 474, 653

van Maanen, A. 1917, PASP, 29, 258

van Maanen, A. 1943, Leaflet of the Astronomical Society of the Pacific, 4, 199

Volk, K., Blum, R., Walker, G., \& Puxley, P. 2003, IAU Circ., 8188, 2 
Vrba, F. J., Henden, A. A., Luginbuhl, C. B., et al. 2004, AJ, 127, 2948

Warren, S. J., Mortlock, D. J., Leggett, S. K., et al. 2007, MNRAS, 381, 1400

Westphal, J. A. 1969, ApJ, 157, L63

Wilson, O. C., \& Vainu Bappu, M. K. 1957, ApJ, 125, 661

Winters, J. G., Henry, T. J., Jao, W.-C., et al. 2011, AJ, 141, 21

Wright, E. L., Skrutskie, M. F., Kirkpatrick, J. D., et al. 2012, arXiv:1203.5764

Wright, E. L., et al. 2010, AJ, 140, 1868

York, D. G., Adelman, J., Anderson, J. E., Jr., et al. 2000, AJ, 120, 1579

Zapatero Osorio, M. R., Béjar, V. J. S., Martín, E. L., et al. 2002, ApJ, 578, 536

Zuckerman, B., \& Song, I. 2004, ARA\&A, 42, 685 
Table 1. WISE All-Sky Release Photometry of WISE Y Dwarfs

\begin{tabular}{|c|c|c|c|c|c|c|c|}
\hline $\begin{array}{c}\text { WISE Designation } \\
\text { (1) }\end{array}$ & $\begin{array}{c}\mathrm{W} 1 \\
(\mathrm{mag}) \\
(2)\end{array}$ & $\begin{array}{c}\text { W2 } \\
(\mathrm{mag}) \\
(3)\end{array}$ & $\begin{array}{c}\text { W3 } \\
(\mathrm{mag}) \\
(4)\end{array}$ & $\begin{array}{c}\mathrm{W} 4 \\
(\mathrm{mag}) \\
(5)\end{array}$ & $\begin{array}{c}\text { W1-W2 } \\
(\mathrm{mag}) \\
(6)\end{array}$ & $\begin{array}{l}\text { W2-W3 } \\
(\mathrm{mag}) \\
(7)\end{array}$ & $\begin{array}{l}\text { Spec. } \\
\text { Type } \\
(8)\end{array}$ \\
\hline WISE J014656.66+423410.0 & $>18.99$ & $15.08 \pm 0.07$ & $13.20 \pm 0.51$ & $>9.41$ & $>3.91$ & $1.88 \pm 0.51$ & Y0 \\
\hline WISE J035000.32-565830.2 & $>18.90$ & $14.73 \pm 0.06$ & $12.53 \pm 0.29$ & $>9.00$ & $>4.17$ & $2.20 \pm 0.30$ & Y1 \\
\hline WISE J035934.06-540154.6 & $>19.29$ & $15.42 \pm 0.07$ & $>13.02$ & $>9.76$ & $>3.87$ & $<2.40$ & Y0 \\
\hline WISE J041022.71+150248.4 $4^{\mathrm{a}}$ & $>18.33$ & $14.18 \pm 0.06$ & $>11.86$ & $>8.90$ & $>4.15$ & $<2.32$ & YO \\
\hline WISE J053516.80-750024.9 & $>18.92$ & $15.06 \pm 0.07$ & $13.12 \pm 0.45$ & $>9.00$ & $>3.86$ & $1.94 \pm 0.46$ & $\geq \mathrm{Y} 1:$ \\
\hline WISE J071322.55-291751.9 & $>18.35$ & $14.48 \pm 0.06$ & $12.67 \pm 0.43$ & $>9.22$ & $>3.87$ & $1.81 \pm 0.43$ & Y0 \\
\hline WISE J073444.02-715744.0 & $>19.42$ & $15.36 \pm 0.06$ & $13.65 \pm 0.42$ & $9.65 \pm 0.33$ & $>4.06$ & $1.71 \pm 0.42$ & Yo \\
\hline WISE J140518.39+553421.3 ${ }^{\mathrm{a}}$ & $>18.82$ & $14.10 \pm 0.04$ & $12.43 \pm 0.27$ & $>9.40$ & $>4.72$ & $1.67 \pm 0.27$ & Yo pec? \\
\hline WISE J154151.65-225024.9 a & $16.74 \pm 0.17^{\mathrm{b}}$ & $14.25 \pm 0.06$ & $>12.31$ & $>8.89$ & $2.49 \pm 0.18^{\mathrm{b}}$ & $<1.94$ & Y0.5 \\
\hline WISE J173835.53+273259.0 a & $>18.41$ & $14.55 \pm 0.06$ & $11.93 \pm 0.19$ & $>8.98$ & $>3.86$ & $2.62 \pm 0.20$ & Y0 \\
\hline WISE J182831.08+265037. $7^{\mathrm{a}}$ & $>18.47$ & $14.39 \pm 0.06$ & $>12.53$ & $>8.75$ & $>4.08$ & $<1.86$ & $\geq \mathrm{Y}_{2}$ \\
\hline WISE J205628.91+145953.2 $2^{\mathrm{a}}$ & $>18.25$ & $13.93 \pm 0.05$ & $12.00 \pm 0.27$ & $>8.78$ & $>4.32$ & $1.93 \pm 0.27$ & Yo \\
\hline WISE J222055.31-362817.4 & $>18.65$ & $14.66 \pm 0.06$ & $>12.81$ & $>9.30$ & $>3.99$ & $>1.85$ & Yo \\
\hline
\end{tabular}

${ }^{a}$ For details about these sources, please see Cushing et al. (2011).

${ }^{\mathrm{b}}$ This source has a bluer W1-W2 color than it did in earlier WISE processing - W1-W2=2.49 \pm 0.18 now versus W1-W2>3.04 before (Kirkpatrick et al. 2011). In both cases, the strong W2 detection would have driven the photometry, but in the newer processing, the code appears to have erroneously assigned to this object some of the W1 flux from the bright star to the north, therefore producing a brighter W1 detection for WISE 1541-2250 than actually exists. 
Table 2. Photometric Follow-up of Y Dwarfs

\begin{tabular}{|c|c|c|c|c|c|c|c|c|c|c|c|}
\hline $\begin{array}{l}\text { WISE Designation } \\
\text { (1) }\end{array}$ & $\begin{array}{l}\text { F140W } \\
(\mathrm{mag}) \\
(2)\end{array}$ & $\begin{array}{c}J \\
(\mathrm{mag}) \\
(3)\end{array}$ & $\begin{array}{c}H \\
(\mathrm{mag}) \\
(4)\end{array}$ & $\begin{array}{l}\text { Ref } \\
(5)\end{array}$ & $\begin{array}{c}\operatorname{ch} 1 \\
(\operatorname{mag}) \\
(6)\end{array}$ & $\begin{array}{c}\mathrm{ch} 2 \\
(\mathrm{mag}) \\
(7)\end{array}$ & $\begin{array}{c}\text { ch1-ch2 } \\
(\mathrm{mag}) \\
(8)\end{array}$ & $\begin{array}{c}\text { F140W-W2 } \\
(\mathrm{mag}) \\
(9)\end{array}$ & $\begin{array}{c}J-\mathrm{W} 2 \\
(\mathrm{mag}) \\
(10)\end{array}$ & $\begin{array}{c}H-\mathrm{W} 2 \\
(\mathrm{mag}) \\
(11)\end{array}$ & $\begin{array}{l}\text { Spec. } \\
\text { Type } \\
(12)\end{array}$ \\
\hline WISE J014656.66+423410.0 & - & $19.40 \pm 0.25$ & $18.71 \pm 0.24$ & 1 & $17.50 \pm 0.07$ & $15.08 \pm 0.02$ & $2.42 \pm 0.07$ & - & $4.32 \pm 0.26$ & $3.63 \pm 0.25$ & Yo \\
\hline WISE J035000.32-565830.2 & $22.30 \pm 0.20$ & $>22.8$ & $>21.5$ & 2 & $17.94 \pm 0.10$ & $14.69 \pm 0.02$ & $3.25 \pm 0.10$ & $7.57 \pm 0.21$ & $>8.1$ & $>6.8$ & Y1 \\
\hline WISE J035934.06-540154.6 & $21.84 \pm 0.20$ & $21.56 \pm 0.24$ & $22.20 \pm 0.43$ & 2 & $17.55 \pm 0.07$ & $15.33 \pm 0.02$ & $2.22 \pm 0.08$ & $6.42 \pm 0.21$ & $6.14 \pm 0.25$ & $6.78 \pm 0.44$ & YO \\
\hline WISE J041022.71+150248.4 & & $19.24 \pm 0.05^{\mathrm{a}}$ & $19.05 \pm 0.09$ & 3 & $16.64 \pm 0.04$ & $14.18 \pm 0.02$ & $2.46 \pm 0.05$ & & $5.06 \pm 0.08$ & $4.87 \pm 0.11$ & Y0 \\
\hline WISE J053516.80-750024.9 & $22.42 \pm 0.20$ & $>21.1$ & $>21.6$ & 2 & $17.77 \pm 0.09$ & $15.01 \pm 0.02$ & $2.76 \pm 0.09$ & $7.36 \pm 0.21$ & $>6.0$ & $>6.5$ & $\geq \mathrm{Y} 1:$ \\
\hline \multirow[t]{2}{*}{ WISE J071322.55-291751.9 } & - & $19.64 \pm 0.15$ & $>19.3$ & 4 & $16.67 \pm 0.04$ & $14.22 \pm 0.02$ & $2.45 \pm 0.05$ & - & $5.16 \pm 0.16$ & $>4.8$ & Yo \\
\hline & - & $20.06 \pm 0.21$ & $21.16 \pm 0.66$ & 10 & & & & - & $5.58 \pm 0.22$ & $6.68 \pm 0.66$ & \\
\hline WISE J073444.02-715744.0 & - & $20.41 \pm 0.27$ & - & 5 & $17.69 \pm 0.08$ & $15.21 \pm 0.02$ & $2.47 \pm 0.08$ & - & $5.05 \pm 0.28$ & - & Y0 \\
\hline WISE J140518.39+553421.3 & $21.22 \pm 0.20$ & $20.09 \pm 0.12$ & $>20.5^{\mathrm{a}}$ & 6 & $16.88 \pm 0.05$ & $14.06 \pm 0.02$ & $2.82 \pm 0.05$ & $7.12 \pm 0.20$ & $5.99 \pm 0.13$ & $>6.4$ & Y0 pec? \\
\hline WISE J154151.65-225024.9 & & $20.74 \pm 0.31^{\mathrm{a}}$ & $>20.2^{\mathrm{a}}$ & 5 & $16.73 \pm 0.04$ & $14.23 \pm 0.02$ & $2.50 \pm 0.05$ & & $6.49 \pm 0.32$ & $>6.0$ & Y 0.5 \\
\hline WISE J173835.53+273259.0 & $19.87 \pm 0.20$ & $19.51 \pm 0.08^{\mathrm{a}}$ & $20.39 \pm 0.33^{\mathrm{a}}$ & 3 & $17.10 \pm 0.05$ & $14.48 \pm 0.02$ & $2.62 \pm 0.06$ & $5.32 \pm 0.21$ & $4.96 \pm 0.10$ & $5.84 \pm 0.34$ & Yo \\
\hline WISE J182831.08+265037.7 & $23.12 \pm 0.20$ & $23.57 \pm 0.35$ & $22.85 \pm 0.24$ & 7 & $16.92 \pm 0.02$ & $14.32 \pm 0.02$ & $2.60 \pm 0.03$ & $8.73 \pm 0.21$ & $9.18 \pm 0.36$ & $8.46 \pm 0.25$ & $\geq \mathrm{Y} 2$ \\
\hline WISE J205628.91+145953.2 & $19.56 \pm 0.20$ & $19.23 \pm 0.13^{\mathrm{a}}$ & $19.62 \pm 0.31^{\mathrm{a}}$ & 8 & $16.04 \pm 0.03$ & $13.92 \pm 0.02$ & $2.11 \pm 0.04$ & $5.63 \pm 0.21$ & $5.30 \pm 0.14$ & $5.69 \pm 0.31$ & Yo \\
\hline WISE J222055.31-362817.4 & - & $20.38 \pm 0.17$ & $20.81 \pm 0.30$ & 9 & $17.20 \pm 0.06$ & $14.73 \pm 0.02$ & $2.46 \pm 0.06$ & - & $5.72 \pm 0.18$ & $6.15 \pm 0.31$ & Y0 \\
\hline
\end{tabular}

Note. - All $J$ and $H$ data are on the MKO-NIR filter system except for those in italics. See text for details.

Note. - References for photometry: (1) Bigelow/2MASS on 2010 Nov 20 UT; (2) Magellan/PANIC on 2010 Nov 25 UT except for the J-band measurement of WISE 0359-5401, which was taken on 2010 Aug 01 UT; (3) Palomar/WIRC on 2010 Jul 26 except for the $J$ measurement of WISE 0410+1502, which was taken on 2010 Aug 29 UT; (4) SOAR/Spartan-IRC on 2012 Jan 13 UT; (5) CTIO4m/NEWFIRM on 2011 Apr 17 UT; (6) Palomar/WIRC combined $-J$ is from 2010 Jul 26 UT and $H$ is from a mosaic made from data taken on 2010 Jul 26 , Aug 28 , SOAR/OSIRIS on 2011 Sep 18 UT for $H$; (10) SOAR/OSIRIS on 2012 Mar 13 UT for $J$ and 2012 Mar 12 UT for $H$.

${ }^{a}$ These values have been updated using of an aperture with radius set to 1.5 times the full width at half maximum of the observed point spread function, as opposed to the use of a standard, fixed aperture size for all observations, as had been done in Cushing et al. 2011 . 
Table 3. New Follow-up Spectroscopy of Y Dwarfs

\begin{tabular}{|c|c|c|c|c|}
\hline $\begin{array}{c}\text { WISE Designation } \\
\text { (1) }\end{array}$ & $\begin{array}{c}\text { Instrument } \\
(2)\end{array}$ & $\begin{array}{l}\text { Obs. date } \\
\text { (UT) } \\
\text { (3) }\end{array}$ & $\begin{array}{l}\text { Int. Time } \\
\quad(\mathrm{sec}) \\
\quad(4)\end{array}$ & $\begin{array}{l}\text { Spec. } \\
\text { Type } \\
(5)\end{array}$ \\
\hline \multirow[t]{2}{*}{ WISE J014656.66+423410.0 } & Keck/NIRSPEC-N3 & 2011 Aug 08 & 6000 & Yo \\
\hline & Keck/NIRSPEC-N5 & 2011 Sep 06 & 3600 & Yo \\
\hline WISE J035000.32-565830.2 & HST/WFC3 & 2011 Aug 13 & 2212 & Y1 \\
\hline WISE J035934.06-540154.6 & $\mathrm{HST} / \mathrm{WFC} 3$ & 2011 Aug 09-10 & 2212 & Y0 \\
\hline WISE J053516.80-750024.9 & $\mathrm{HST} / \mathrm{WFC} 3^{\mathrm{a}}$ & 2011 Sep 27 & 2212 & $\geq \mathrm{Y} 1:$ \\
\hline WISE J071322.55-291751.9 & Keck/NIRSPEC-N3 & 2011 Oct 07-09 & 12000 & Y0 \\
\hline WISE J073444.02-715744.0 & Magellan/FIRE & 2012 Jan 05 & 1775 & Yo \\
\hline \multirow[t]{2}{*}{ WISE J205628.91+145953.2 } & Keck/NIRSPEC-N3 & 2011 Aug 09 & $1200^{\mathrm{b}}$ & Y0 \\
\hline & $\mathrm{HST} / \mathrm{WFC} 3$ & 2011 Sep 04 & 2012 & Yo \\
\hline \multirow[t]{2}{*}{ WISE J222055.31-362817.4 } & Keck/NIRSPEC-N3 & 2011 Sep 05/Oct 09 & 5400 & Yo \\
\hline & Keck/NIRSPEC-N5 & 2011 Sep 06 & 3600 & Y0 \\
\hline
\end{tabular}

a This spectrum suffers heavy contamination from a field object.

${ }^{b}$ This spectrum was added to the 2400s NIRSPEC-N3 spectrum previously reported in Kirkpatrick et al. 2011). 
Table 4. The Census of Stars and Brown Dwarfs within 8 Parsecs of the Sun

\begin{tabular}{|c|c|c|c|c|c|c|c|c|c|c|}
\hline $\begin{array}{c}\text { Discovery Name } \\
\text { (1) }\end{array}$ & $\begin{array}{l}\text { Other Name } \\
\text { (2) }\end{array}$ & $\begin{array}{c}\text { Gliese Name } \\
\text { (3) }\end{array}$ & $\begin{array}{l}\pi_{t r i g}{ }^{a} \\
(\text { mas) } \\
(4)\end{array}$ & $\begin{array}{l}\sigma_{\pi} \\
(\mathrm{mas}) \\
(5)\end{array}$ & $\begin{array}{c}\pi \text { Ref. } \\
(6)\end{array}$ & $\begin{array}{l}\text { Spec. } \\
\text { Type } \\
(7)\end{array}$ & $\begin{array}{l}\text { Spec. } \\
\text { Ref. } \\
(8)\end{array}$ & $\begin{array}{c}\text { J2000 Coords. } \\
\text { (9) }\end{array}$ & $\begin{array}{l}\text { Sys. } \\
\text { Rank } \\
(10)\end{array}$ & $\begin{array}{c}\text { Indiv. } \\
\text { Rank } \\
(11)\end{array}$ \\
\hline Sun & Sol & - & - & - & - & $\mathrm{G} 2 \mathrm{~V}$ & 51 & - & 0 & 0 \\
\hline Proxima Cen ${ }^{\mathrm{b}}$ & $\alpha$ Cen $\mathrm{C}$ & Gl 551 & 769.91 & 0.54 & 16 & $\mathrm{M} 5.5 \mathrm{~V}$ & 18 & $1429-6240$ & 1 & 1 \\
\hline Rigel Kentaurus A & $\alpha$ Cen A & G1 $559 \mathrm{~A}$ & 754.81 & 4.11 & 5 & $\mathrm{G} 2 \mathrm{~V}$ & 50 & $1439-6050$ & 1 & 2 \\
\hline Rigel Kentaurus B & $\alpha$ Cen B & G1 559 B & - & - & - & $\mathrm{K} 2 \mathrm{IV} \mathrm{C} 2+1$ & 50 & $1439-6050$ & 1 & 2 \\
\hline Barnard's Star & - & Gl 699 & 545.4 & 0.3 & 16 & M4 V & 38 & $1757+0441$ & 2 & 4 \\
\hline Wolf 359 & - & Gl 406 & 419.1 & 2.1 & 6 & M6 V & 38 & $1056+0700$ & 3 & 5 \\
\hline Lalande 21185 & - & Gl 411 & 392.64 & 0.67 & 5 & $\mathrm{M} 2 \mathrm{~V}$ & 38 & $1103+3558$ & 4 & 6 \\
\hline Sirius A & $\alpha \mathrm{CMa} \mathrm{A}$ & Gl $244 \mathrm{~A}$ & 379.21 & 1.58 & 5 & $\mathrm{~A} 0 \mathrm{~mA} 1 \mathrm{Va}$ & 49 & $0645-1642$ & 5 & 7 \\
\hline Sirius B & $\alpha \mathrm{CMa} \mathrm{B}$ & G1 244 B & - & - & - & $\mathrm{DA} 2$ & 52 & $0645-1643$ & 5 & 7 \\
\hline L 726-8 A & BL Cet & Gl $65 \mathrm{~A}$ & 373.7 & 2.7 & 6 & $\mathrm{M} 5.5 \mathrm{~V}$ & 38 & $0139-1757$ & 6 & 9 \\
\hline L $726-8$ B & UV Cet & G1 $65 \mathrm{~B}$ & - & - & - & M6 V & 38 & $0139-1757$ & 6 & 9 \\
\hline Ross 154 & - & G1 729 & 336.72 & 2.03 & 5 & $\mathrm{M} 3.5 \mathrm{~V}$ & 21 & $1849-2350$ & 7 & 11 \\
\hline Ross 248 & - & Gl 905 & 316.0 & 1.1 & 6 & M5.5 V & 21 & $2341+4410$ & 8 & 12 \\
\hline$\epsilon$ Eri & - & Gl 144 & 310.94 & 0.16 & 5 & K2 V (k) & 50 & $0332-0927$ & 9 & 13 \\
\hline Lacaille 9352 & - & Gl 887 & 305.26 & 0.70 & 5 & $\mathrm{M} 0.5 \mathrm{~V}$ & 44 & $2305-3551$ & 10 & 14 \\
\hline Ross 128 & - & Gl 447 & 298.2 & 1.7 & 6 & M4 V & 21 & $1147+0048$ & 11 & 15 \\
\hline L 789-6 A & - & Gl $866 \mathrm{~A}$ & 289.5 & 4.4 & 6 & $M 5^{\mathrm{c}} \mathrm{V}$ & 21 & $2238-1517$ & 12 & 16 \\
\hline L 789-6 B & - & Gl 866 B & - & - & - & $\mathrm{M} 5.5 \mathrm{~d}^{\mathrm{d}} \mathrm{V}$ & 45 & $2238-1515$ & 12 & 16 \\
\hline L 789-6 C & - & Gl $866 \mathrm{C}$ & - & - & - & $\mathrm{M} 6.5 \mathrm{:}^{\mathrm{d}} \mathrm{V}$ & 45 & $2238-1517$ & 12 & 16 \\
\hline 61 Cyg A & - & Gl $820 \mathrm{~A}$ & 286.9 & 1.1 & 6 & K5 V & 38 & $2106+3844$ & 13 & 19 \\
\hline 61 Cyg B & - & Gl $820 \mathrm{~B}$ & - & - & - & K7 V & 38 & $2106+3844$ & 13 & 19 \\
\hline Procyon A & $\alpha \mathrm{CMi} \mathrm{A}$ & G1 $280 \mathrm{~A}$ & 284.56 & 1.26 & 5 & F5 IV-V & 49 & $0739+0513$ & 14 & 21 \\
\hline Procyon B & $\alpha$ CMi B & G1 $280 \mathrm{~B}$ & & - & - & DA & 52 & $0739+0515$ & 14 & 21 \\
\hline$\Sigma 2398 \mathrm{~A}$ & - & G1 $725 \mathrm{~A}$ & 280.18 & 2.18 & 5 & M3 V & 41 & $1842+5937$ & 15 & 23 \\
\hline$\Sigma 2398 \mathrm{~B}$ & - & Gl 725 B & - & - & - & M3.5 V & 41 & $1842+5937$ & 15 & 23 \\
\hline Groombridge $34 \mathrm{~A}$ & - & G1 $15 \mathrm{~A}$ & 278.76 & 0.77 & 5 & $\mathrm{M} 1.5 \mathrm{~V}$ & 21 & $0018+4401$ & 16 & 25 \\
\hline Groombridge $34 \mathrm{~B}$ & - & Gl 15 B & & - & - & M3.5 V & 21 & $0018+4401$ & 16 & 25 \\
\hline$\epsilon$ Ind $\mathrm{A}$ & - & Gl $845 \mathrm{~A}$ & 276.06 & 0.28 & 5 & K4 V (k) & 50 & $2203-5647$ & 17 & 27 \\
\hline$\epsilon$ Ind $\mathrm{Ba}$ & - & Gl $845 \mathrm{Ba}$ & - & - & - & $\mathrm{T} 1$ & 53 & $2204-5646$ & 17 & 27 \\
\hline$\epsilon$ Ind $\mathrm{Bb}$ & - & Gl $845 \mathrm{Bb}$ & - & - & - & $\mathrm{T} 6$ & 53 & $2204-5646$ & 17 & 27 \\
\hline G $51-15$ & - & GJ 1111 & 275.8 & 3.0 & 6 & $\mathrm{M} 6.5 \mathrm{~V}$ & 38 & $0829+2646$ & 18 & 30 \\
\hline$\tau$ Cet & - & G1 71 & 273.96 & 0.17 & 5 & G8.5 V & 50 & $0144-1556$ & 19 & 31 \\
\hline L $372-58$ & - & GJ 1061 & 271.92 & 1.34 & 12 & M5 V & 12 & $0335-4430$ & 20 & 32 \\
\hline L $725-32$ & - & G1 54.1 & 268.8 & 3.2 & 6 & $\mathrm{M} 4.5 \mathrm{~V}$ & 21 & $0112-1659$ & 21 & 33 \\
\hline Luyten's Star & $\mathrm{BD}+051668$ & G1 273 & 262.98 & 1.39 & 5 & $\mathrm{M} 3.5 \mathrm{~V}$ & 38 & $0727+0513$ & 22 & 34 \\
\hline SCR J1845-6357 A & - & - & 259.45 & 1.11 & 12 & $\mathrm{M} 8.5 \mathrm{~V}$ & 12 & $1845-6357$ & 23 & 35 \\
\hline SCR J1845-6357 B & - & - & - & - & - & $\mathrm{T} 6$ & 54 & $1845-6357$ & 23 & 35 \\
\hline Teegarden's Star & SO J025300.5+165258 & - & 259.25 & 0.94 & 74 & $\mathrm{M} 6 \mathrm{~V}$ & 12 & $0253+1652$ & 24 & 37 \\
\hline Kapteyn's Star & $\mathrm{ZC} 5 \mathrm{~h} 243^{\mathrm{bb}}$ & Gl 191 & 255.66 & 0.91 & 5 & sdM1 & 44 & $0511-4501$ & 25 & 38 \\
\hline Lacaille 8760 & - & Gl 825 & 253.41 & 0.80 & 5 & K7 V & 44 & $2117-3852$ & 26 & 39 \\
\hline Krüger $60 \mathrm{~A}$ & - & Gl $860 \mathrm{~A}$ & 251.5 & 3.7 & 6 & M3 V & 39 & $2228+5741$ & 27 & 40 \\
\hline Krüger $60 \mathrm{~B}$ & - & Gl $860 \mathrm{~B}$ & - & - & - & M4 V & 39 & $2227+5741$ & 27 & 40 \\
\hline DENIS-P J104814.7-395606.1 & - & - & 247.71 & 1.55 & 17 & $\mathrm{M} 8.5 \mathrm{~V}$ & 20 & $1048-3956$ & 28 & 42 \\
\hline UGPS J072227.51-054031.2 & - & - & 246 & 33 & 22 & T9 & 4 & $0722-0540$ & 29 & 43 \\
\hline Ross $614 \mathrm{~A}$ & - & G1 $234 \mathrm{~A}$ & 242.32 & 3.12 & 5 & M4-4.5 V & 42 & $0629-0248$ & 30 & 44 \\
\hline Ross 614 B & - & G1 234 B & - & - & - & $\mathrm{M} 4.5-6.5 \mathrm{~V}$ & 42 & $0629-0248$ & 30 & 44 \\
\hline WISE J035000.32-565830.2 & - & - & 238 & 38 & 2 & $\mathrm{Y} 1$ & 1 & $0350-5658$ & 31 & 46 \\
\hline WISEPA J154151.66-225025.2 & - & - & $238^{\mathrm{u} u}$ & - & 1 & Y0.5 & 1 & $1541-2250$ & 32 & 47 \\
\hline
\end{tabular}


Table 4-Continued

\begin{tabular}{|c|c|c|c|c|c|c|c|c|c|c|}
\hline $\begin{array}{c}\text { Discovery Name } \\
\text { (1) }\end{array}$ & $\begin{array}{l}\text { Other Name } \\
\text { (2) }\end{array}$ & $\begin{array}{c}\text { Gliese Name } \\
\text { (3) }\end{array}$ & $\begin{array}{l}\pi_{t r i g}{ }^{a} \\
(\mathrm{mas}) \\
(4)\end{array}$ & $\begin{array}{l}\sigma_{\pi} \\
(\mathrm{mas}) \\
(5)\end{array}$ & $\begin{array}{c}\pi \text { Ref. } \\
(6)\end{array}$ & $\begin{array}{l}\text { Spec. } \\
\text { Type } \\
(7)\end{array}$ & $\begin{array}{l}\text { Spec. } \\
\text { Ref. } \\
(8)\end{array}$ & $\begin{array}{c}\text { J2000 Coords. } \\
\text { (9) }\end{array}$ & $\begin{array}{l}\text { Sys. } \\
\text { Rank } \\
(10)\end{array}$ & $\begin{array}{l}\text { Indiv. } \\
\text { Rank } \\
(11)\end{array}$ \\
\hline BD-12 4523 & Wolf 1061 & G1 628 & 232.98 & 1.60 & 5 & M3 V & 21 & $1630-1239$ & 33 & 48 \\
\hline van Maanen's Star ${ }^{\mathrm{e}}$ & - & G1 35 & 232.5 & 1.9 & 6 & DZ8 & 52 & $0049+0523$ & 34 & 49 \\
\hline Gou $32416^{\mathrm{bb}}$ & $\mathrm{CD}-3715492$ & G1 1 & 230.42 & 0.90 & 5 & $\mathrm{M} 1.5 \mathrm{~V}$ & 44 & $0005-3721$ & 35 & 50 \\
\hline Wolf $424 \mathrm{~A}$ & - & Gl $473 \mathrm{~A}$ & 227.9 & 4.6 & 6 & M5.5* V & 40 & $1233+0901$ & 36 & 51 \\
\hline Wolf $424 \mathrm{~B}$ & - & G1 473 B & - & - & - & M5.5: V & 46 & $1233+0901$ & 36 & 51 \\
\hline L $1159-16$ & - & G1 83.1 & 224.8 & 2.9 & 6 & M $4.5 \mathrm{~V}$ & 38 & $0200+1303$ & 37 & 53 \\
\hline Oe Arg $17415-6^{\mathrm{b} b}$ & $\mathrm{BD}+68946$ & G1 687 & 220.84 & 0.94 & 5 & M3 V & 21 & $1736+6820$ & 38 & 54 \\
\hline LP 731-58 & LHS 292 & - & 220.3 & 3.6 & 6 & $\mathrm{M} 6.5 \mathrm{~V}$ & 40 & $1048-1120$ & 39 & 55 \\
\hline Innes $\left(\right.$ unnumbered) ${ }^{\mathrm{g}}$ & $\mathrm{CD}-4611540$ & Gl 674 & 220.24 & 1.42 & 5 & $\mathrm{M} 2.5 \mathrm{~V}$ & 44 & $1728-4653$ & 40 & 56 \\
\hline G $208-44$ A & - & GJ $1245 \mathrm{~A}$ & 220.2 & 1.0 & 6 & M $5.5^{\mathrm{h}} \mathrm{V}$ & 41 & $1953+4424$ & 41 & 57 \\
\hline G $208-44$ B & - & GJ $1245 \mathrm{C}$ & - & - & - & $\mathrm{M} 6.5 \mathrm{i}^{\mathrm{i}} \mathrm{V}$ & 1 & $1953+4424$ & 41 & 57 \\
\hline G $208-45$ & - & GJ $1245 \mathrm{~B}$ & - & - & - & M $5.5 \mathrm{~V}$ & 41 & $1953+4424$ & 41 & 57 \\
\hline Innes (unnumbered) ${ }^{g}$ & Ci 20658 & Gl 440 & 215.80 & 1.25 & 8 & DQ6 & 52 & $1145-6450$ & 42 & 60 \\
\hline Ross 780 & - & G1 876 & 213.28 & 2.12 & 5 & $\mathrm{M} 3.5 \mathrm{~V}$ & 21 & $2253-1415$ & 43 & 61 \\
\hline G $158-27$ & - & GJ 1002 & 213.0 & 3.6 & 6 & $\mathrm{M} 5.5 \mathrm{~V}$ & 21 & $0006-0732$ & 44 & 62 \\
\hline L $143-23^{j}$ & LHS 288 & - & 208.95 & 2.73 & 12 & M5 V & 12 & $1044-6112$ & 45 & 62 \\
\hline WISEPC J140518.40+553421.4 & - & - & 207 & 39 & 2 & YOp & 4 & $1405+5534$ & 46 & 62 \\
\hline Lalande $21258 \mathrm{~A}$ & - & G1 $412 \mathrm{~A}$ & 206.27 & 1.00 & 5 & M1 V & 21 & $1105+4331$ & 47 & 65 \\
\hline Lalande 21258 B & - & G1 412 B & - & - & - & M $5.5 \mathrm{~V}$ & 21 & $1105+4331$ & 47 & 65 \\
\hline Groombridge 1618 & - & G1 380 & 205.21 & 0.54 & 5 & $\mathrm{~K} 7 \mathrm{~V}$ & 38 & $1011+4927$ & 48 & 67 \\
\hline $\mathrm{BD}+202465$ & G $54-23$ & G1 388 & 204.6 & 2.8 & 6 & M3 V & 21 & $1019+1952$ & 49 & 68 \\
\hline DENIS J081730.0-615520 & & - & 203 & 13 & 23 & $\mathrm{~T} 6$ & 23 & $0817-6155$ & 50 & 69 \\
\hline $\mathrm{CD}-4913515$ & L $354-89$ & Gl 832 & 201.87 & 1.01 & 5 & $\mathrm{M} 1.5 \mathrm{~V}$ & 44 & $2133-4900$ & 51 & 70 \\
\hline LP 944-20 & - & - & 201.4 & 4.2 & 27 & M9 V & 48 & $0339-3525$ & 52 & 71 \\
\hline DENIS-P J025503.3-470049 & - & - & 201.37 & 3.89 & 13 & L8 & 55 & $0255-4700$ & 53 & 72 \\
\hline$o^{2}$ Eri A & - & Gl $166 \mathrm{~A}$ & 200.62 & 0.23 & 5 & $\mathrm{~K} 0.5 \mathrm{~V}$ & 50 & $0415-0739$ & 54 & 73 \\
\hline$o^{2}$ Eri B & - & G1 166 B & - & - & - & DA4 4 & 52 & $0415-0739$ & 54 & 73 \\
\hline$o^{2}$ Eri C & - & Gl $166 \mathrm{C}$ & - & - & - & $\mathrm{M} 4.5 \mathrm{~V}$ & 38 & $0415-0739$ & 54 & 73 \\
\hline Innes (unnumbered) ${ }^{g}$ & $\mathrm{CD}-4411909$ & G1 682 & 196.90 & 2.15 & 5 & $\mathrm{M} 3.5 \mathrm{~V}$ & 44 & $1737-4419$ & 55 & 76 \\
\hline 70 Oph A & - & G1 $702 \mathrm{~A}$ & 196.72 & 0.83 & 5 & KO- V & 49 & $1805+0229$ & 56 & 77 \\
\hline $70 \mathrm{Oph} \mathrm{B}$ & - & G1 $702 \mathrm{~B}$ & - & 0.83 & 5 & K5 V & 56 & $1805+0229$ & 56 & 77 \\
\hline $\mathrm{BD}+434305$ & LFT 1737 & G1 873 & 195.22 & 1.87 & 5 & $\mathrm{M} 3.5 \mathrm{~V}$ & 42 & $2246+4420$ & 57 & 79 \\
\hline Altair & $\alpha \mathrm{Aql}$ & G1 768 & 194.95 & 0.57 & 5 & $\mathrm{~A} 7 \mathrm{Vn}$ & 49 & $1950+0852$ & 58 & 80 \\
\hline WISEPC J150649.97+702736.0 & - & - & 193 & 26 & 2 & T6 & 3 & $1506+7027$ & 59 & 81 \\
\hline WISEPC J205628.90+145953.3 & - & - & 192 & - & 1 & Yo & 4 & $2056+1459$ & 60 & 82 \\
\hline G 9-38 A & - & GJ $1116 \mathrm{~A}$ & 191.2 & 2.5 & 6 & M $5.5 \mathrm{~V}$ & 57 & $0858+1945$ & 61 & 83 \\
\hline G 9-38 B & - & GJ $1116 \mathrm{~B}$ & - & - & - & M $5.5 \mathrm{~V}$ & 57 & $0858+1945$ & 61 & 83 \\
\hline G $99-49$ & - & - & 190.93 & 1.89 & 12 & $\mathrm{M} 3.5 \mathrm{~V}$ & 21 & $0600+0242$ & 62 & 85 \\
\hline LP $656-38$ & LHS 1723 & - & 187.92 & 1.26 & 12 & M4 V & 12 & $0501-0656$ & 63 & 86 \\
\hline 2MASS J09393548-2448279 & & - & 187.3 & 4.6 & 28 & T8 & 53 & $0939-2448$ & 64 & 87 \\
\hline $\mathrm{AC}+793888$ & LFT 849 & Gl 445 & 186.86 & 1.70 & 5 & M3.5 V & 21 & $1147+7841$ & 65 & 88 \\
\hline Lalande 25372 & - & G1 526 & 185.49 & 1.10 & 5 & M1.5 V & 21 & $1345+1453$ & 66 & 89 \\
\hline Stein $2051 \mathrm{~A}$ & - & Gl $169.1 \mathrm{~A}$ & 180.6 & 0.8 & 6 & M4 V & 42 & $0431+5858$ & 67 & 90 \\
\hline Stein 2051 B & - & Gl 169.1B & - & - & - & DC5 & 52 & $0431+5858$ & 67 & 90 \\
\hline Wolf 294 & - & Gl 251 & 179.01 & 1.60 & 5 & M3 V & 38 & $0654+3316$ & 68 & 92 \\
\hline 2MASSI J0415195-093506 & - & & 177.3 & 2.2 & 76 & T8 & 53 & $0415-0935$ & 69 & 93 \\
\hline Strb. $1611^{\mathrm{s}}$ & Wolf 1453 & Gl 205 & 176.77 & 1.18 & 5 & $\mathrm{M} 1.5 \mathrm{~V}$ & 38 & $0531-0340$ & 70 & 94 \\
\hline 2MASSI J1835379+325954 & LSR J1835+3259 & - & 176.5 & 0.5 & 31 & $\mathrm{M} 8.5 \mathrm{~V}$ & 31 & $1835+3259$ & 71 & 95 \\
\hline
\end{tabular}


Table 4-Continued

\begin{tabular}{|c|c|c|c|c|c|c|c|c|c|c|}
\hline $\begin{array}{c}\text { Discovery Name } \\
\text { (1) }\end{array}$ & $\begin{array}{l}\text { Other Name } \\
\text { (2) }\end{array}$ & $\begin{array}{c}\text { Gliese Name } \\
\text { (3) }\end{array}$ & $\begin{array}{l}\pi_{t r i g}{ }^{a} \\
(\operatorname{mas}) \\
(4)\end{array}$ & $\begin{array}{l}\sigma_{\pi} \\
(\mathrm{mas})\end{array}$ & $\begin{array}{c}\pi \text { Ref. } \\
(6)\end{array}$ & $\begin{array}{l}\text { Spec. } \\
\text { Type } \\
(7)\end{array}$ & $\begin{array}{l}\text { Spec. } \\
\text { Ref. } \\
(8)\end{array}$ & $\begin{array}{l}\text { J2000 Coords. } \\
\text { (9) }\end{array}$ & $\begin{array}{l}\text { Sys. } \\
\text { Rank } \\
(10)\end{array}$ & $\begin{array}{l}\text { Indiv. } \\
\text { Rank } \\
(11)\end{array}$ \\
\hline WISEPA J174124.26+255319.5 & - & - & 176 & 22 & 2 & T9 & 3 & $1741+2553$ & 72 & 96 \\
\hline LP $816-60$ & - & - & 175.03 & 3.40 & 5 & M4 V & 50 & $2052-1658$ & 73 & 97 \\
\hline L $449-1$ & - & - & 175 & - & 14 & M4 V & 14 & $0517-3521$ & 74 & 98 \\
\hline L $668-21$ A & - & G1 $229 \mathrm{~A}$ & 173.81 & 0.99 & 5 & M1 V & 38 & $0610-2151$ & 75 & 99 \\
\hline L $668-21 \mathrm{~B}$ & - & G1 $229 \mathrm{~B}$ & - & - & - & $\mathrm{T} 7 \mathrm{pec}$ & 53 & $0610-2152$ & 75 & 99 \\
\hline$\sigma$ Dra & - & G1 764 & 173.77 & 0.18 & 5 & G9 V & 49 & $1932+6939$ & 76 & 101 \\
\hline Ross 47 & - & G1 213 & 171.55 & 3.99 & 5 & M4 V & 38 & $0542+1229$ & 77 & 102 \\
\hline Innes (unnumbered) ${ }^{g}$ & - & G1 693 & 171.48 & 2.31 & 5 & $\mathrm{M} 2 \mathrm{~V}$ & 44 & $1746-5719$ & 78 & 103 \\
\hline Lalande $27173 \mathrm{~A}$ & - & G1 $570 \mathrm{~A}$ & 171.22 & 0.94 & 5 & $\mathrm{~K} 4 \mathrm{~V}$ & 50 & $1457-2124$ & 79 & 104 \\
\hline Lalande 27173 B & - & G1 $570 \mathrm{~B}$ & - & - & - & $\mathrm{M} 11^{\mathrm{m}} \mathrm{V}$ & 21 & $1457-2124$ & 79 & 104 \\
\hline Lalande $27173 \mathrm{C}$ & - & G1 $570 \mathrm{C}$ & - & - & - & $\mathrm{M} 3:^{\mathrm{n}} \mathrm{V}$ & 57 & $1457-2124$ & 79 & 104 \\
\hline 2MASSW J1457150-212148 & - & G1 $570 \mathrm{D}$ & - & - & - & $\mathrm{T} 7.5$ & 53 & $1457-2121$ & 79 & 104 \\
\hline Wolf 1055 & - & G1 $752 \mathrm{~A}$ & 171.2 & 0.7 & 6 & M3 V & 38 & $1916+0510$ & 80 & 108 \\
\hline van Biesbroeck 10 & - & G1 752 B & - & - & - & M8 V & 41 & $1916+0509$ & 80 & 108 \\
\hline WISE J053516.80-750024.9 & - & - & 170 & 44 & 2 & $\mathrm{ZY} 1:$ & & $0535-7500$ & 81 & 110 \\
\hline L $347-14$ & - & G1 754 & 169.03 & 1.55 & 17 & $\mathrm{M} 3 \mathrm{~V}$ & 19 & $1920-4533$ & 82 & 111 \\
\hline Innes (unnumbered) ${ }^{g}$ & - & G1 588 & 168.66 & 1.30 & 5 & $\mathrm{M} 2.5 \mathrm{~V}$ & 44 & $1532-4116$ & 83 & 112 \\
\hline $36 \mathrm{Oph} A$ & - & Gl $663 \mathrm{~A}$ & 168.54 & 0.54 & 5 & KO V & 59 & $1715-2636$ & 84 & 113 \\
\hline 36 Oph B & - & G1 663 B & - & - & - & K1 V & 59 & $1715-2636$ & 84 & 113 \\
\hline $36 \mathrm{Oph} \mathrm{\textrm {C } ^ { \circ }}$ & - & G1 664 & - & - & - & K5 V (k) & 50 & $1716-2632$ & 84 & 113 \\
\hline$\eta$ Cas A & - & Gl $34 \mathrm{~A}$ & 167.98 & 0.48 & 5 & F9 V & 58 & $0049+5748$ & 85 & 116 \\
\hline$\eta$ Cas B & - & Gl $34 \mathrm{~B}$ & - & - & - & $\mathrm{K} 7 \mathrm{~V}$ & 21 & $0049+5749$ & 85 & 116 \\
\hline Ross 882 & - & Gl 285 & 167.88 & 2.31 & 5 & M4 V & 21 & $0744+0333$ & 86 & 118 \\
\hline Lalande 46650 & - & G1 908 & 167.29 & 1.23 & 5 & M1 V & 21 & $2349+0224$ & 87 & 119 \\
\hline 2MASS J05332802-4257205 & - & - & 167 & - & 32 & $\mathrm{M} 4.5 \mathrm{~V}$ & 32 & $0533-4257$ & 88 & 120 \\
\hline 2MASS J18450079-1409036 & - & - & 167 & - & 32 & M5 V & 32 & $1845-1409$ & 89 & 121 \\
\hline 2MASS J18450097-1409053 & - & - & 167 & - & 32 & M5 V & 32 & $1845-1409$ & 89 & 121 \\
\hline 2MASS J20360829-3607115 & - & - & 167 & - & 32 & $\mathrm{M} 4.5 \mathrm{~V}$ & 32 & $2036-3607$ & 90 & 123 \\
\hline L $722-22$ A & - & GJ $1005 \mathrm{~A}$ & 166.6 & $\sim 2$ & 24 & M3.5 V & 42 & $0015-1607$ & 91 & 124 \\
\hline L $722-22$ B & - & GJ 1005 B & - & - & - & $\mathrm{M} 4.5-6.5 \mathrm{~V}$ & 42 & $0015-1607$ & 91 & 124 \\
\hline LPM 728 A & - & Gl $783 \mathrm{~A}$ & 166.25 & 0.27 & 5 & $\mathrm{~K} 2.5 \mathrm{~V}$ & 50 & $2011-3606$ & 92 & 126 \\
\hline LPM 728 B & - & G1 $783 \mathrm{~B}$ & - & - & - & $\mathrm{M} 3.5 \mathrm{~V}^{\mathrm{q}}$ & 56 & $2011-3606$ & 92 & 126 \\
\hline WISEPA J025409.45+022359.1 & - & - & 166 & 26 & 2 & T8 & 3 & $0254+0223$ & 93 & 128 \\
\hline $82 \mathrm{Eri}$ & - & Gl 139 & 165.47 & 0.19 & 5 & $\mathrm{G} 8 \mathrm{~V}$ & 50 & $0319-4304$ & 94 & 129 \\
\hline Ross 986 A & - & G1 $268 \mathrm{~A}$ & 165.2 & 2.1 & 6 & $\mathrm{M} 4.5^{\mathrm{c}} \mathrm{V}$ & 38 & $0710+3831$ & 95 & 130 \\
\hline Ross 986 B & - & Gl $268 \mathrm{~B}$ & - & - & - & M5: V & 60 & $0710+3832$ & 95 & 130 \\
\hline WISEPA J041022.71+150248.5 & - & - & 164 & 24 & 2 & Yo & 4 & $0410+1502$ & 96 & 132 \\
\hline LP 44-113 & - & GJ 1221 & 164.7 & 2.4 & 6 & DXP9 & 52 & $1748+7052$ & 97 & 133 \\
\hline$\delta$ Pav & - & G1 780 & 163.71 & 0.17 & 5 & G8 IV & 50 & $2008-6610$ & 98 & 134 \\
\hline 2MASSI J0937347+293142 & - & - & 163.39 & 1.76 & 26 & T6 pec & 53 & $0937+2931$ & 99 & 135 \\
\hline $\mathrm{BD}+531320$ & LFT 634 & G1 $338 \mathrm{~A}$ & 162.8 & 2.9 & 6 & MO V & 38 & $0914+5241$ & 100 & 136 \\
\hline $\mathrm{BD}+531321$ & LFT 635 & G1 338 B & - & - & - & K7 V & 38 & $0914+5241$ & 100 & 136 \\
\hline $\mathrm{BD}+195116 \mathrm{~A}$ & LFT 1799 & Gl $896 \mathrm{~A}$ & 161.76 & 1.66 & 5 & $\mathrm{M} 3.5 \mathrm{~V}$ & 21 & $2331+1956$ & 101 & 138 \\
\hline $\mathrm{BD}+195116 \mathrm{~B}$ & LFT 1800 & G1 896 B & & - & - & $\mathrm{M} 4.5 \mathrm{~V}$ & 21 & $2331+1956$ & 101 & 138 \\
\hline LPM 730 & - & G1 784 & 161.34 & 1.00 & 5 & Mo V & 56 & $2013-4509$ & 102 & 140 \\
\hline WISE J014656.66+423410.0 & - & & 159 & - & 1 & Yo & & $0146+4234$ & 103 & 141 \\
\hline Wolf 1481 & - & G1 555 & 158.46 & 2.62 & 17 & $\mathrm{M} 3.5 \mathrm{~V}$ & 21 & $1434-1231$ & 104 & 142 \\
\hline 2MASS J15031961+2525196 & - & - & 157.2 & 2.2 & 76 & T5 & 53 & $1503+2525$ & 105 & 143 \\
\hline
\end{tabular}


Table 4-Continued

\begin{tabular}{|c|c|c|c|c|c|c|c|c|c|c|}
\hline $\begin{array}{c}\text { Discovery Name } \\
\text { (1) }\end{array}$ & $\begin{array}{c}\text { Other Name } \\
\text { (2) }\end{array}$ & $\begin{array}{c}\text { Gliese Name } \\
\text { (3) }\end{array}$ & $\begin{array}{l}\pi_{\text {trig }}{ }^{\mathrm{a}} \\
(\mathrm{mas}) \\
(4)\end{array}$ & $\begin{array}{c}\sigma_{\pi} \\
(\text { mas) } \\
(5)\end{array}$ & $\begin{array}{c}\pi \text { Ref. } \\
(6)\end{array}$ & $\begin{array}{l}\text { Spec. } \\
\text { Type } \\
(7)\end{array}$ & $\begin{array}{l}\text { Spec. } \\
\text { Ref. } \\
(8)\end{array}$ & $\begin{array}{c}\text { J2000 Coords. } \\
\text { (9) }\end{array}$ & $\begin{array}{l}\text { Sys. } \\
\text { Rank } \\
(10)\end{array}$ & $\begin{array}{l}\text { Indiv. } \\
\text { Rank } \\
(11)\end{array}$ \\
\hline LP $368-128$ & LHS 2090 & - & 156.87 & 2.67 & 12 & M6 V & 12 & $0900+2150$ & 106 & 144 \\
\hline L $471-42$ & LHS 337 & - & 156.78 & 1.99 & 12 & M4 V & 12 & $1238-3822$ & 107 & 145 \\
\hline G $99-44$ & - & Gl 223.2 & 156.13 & 0.84 & 8 & DZ9 & 52 & $0555-0410$ & 108 & 146 \\
\hline G $180-60$ & - & - & $156^{\mathrm{r}}$ & 4 & 25 & M5 V & 65 & $1631+4051$ & 109 & 147 \\
\hline SIMP J013656.5+093347 & - & - & 156 & - & 34 & $\mathrm{~T} 2.5$ & 34 & $0136+0933$ & 110 & 148 \\
\hline Wolf $630 \mathrm{~A}$ & - & G1 $644 \mathrm{~A}$ & 154.8 & 0.6 & 6 & $\mathrm{M} 2.5^{\mathrm{t}} \mathrm{V}$ & 21 & $1655-0819$ & 111 & 149 \\
\hline Wolf $630 \mathrm{Ba}$ & - & Gl $644 \mathrm{~B}$ & - & - & - & M3: ${ }^{\mathrm{u}} \mathrm{V}$ & 1 & $1655-0820$ & 111 & 149 \\
\hline van Biesbroeck 8 & - & Gl $644 \mathrm{C}$ & $-^{\mathrm{v}}$ & - & - & M7 V & 41 & $1655-0823$ & 111 & 149 \\
\hline Wolf $630 \mathrm{Bb}$ & - & G1 $644 \mathrm{D}$ & - & - & - & M3.5: ${ }^{\mathrm{u}} \mathrm{V}$ & 1 & $1655-0820$ & 111 & 149 \\
\hline Wolf 629 & - & G1 643 & $-^{\mathrm{w}}$ & - & - & M3.5 V & 38 & $1655-0819$ & 111 & 149 \\
\hline Wolf 562 & $\mathrm{BD}-74003$ & G1 581 & 154.66 & 2.62 & 17 & $\mathrm{M} 2.5 \mathrm{~V}$ & 21 & $1519-0743$ & 112 & 154 \\
\hline $\mathrm{BD}+452505 \mathrm{~A}$ & LFT 1326 & Gl $661 \mathrm{~A}$ & 154.0 & 3.6 & 6 & $\mathrm{M}^{\mathrm{c}} \mathrm{V}$ & 39 & $1710+4542$ & 113 & 155 \\
\hline $\mathrm{BD}+452505 \mathrm{~B}$ & LFT 1327 & Gl $661 \mathrm{~B}$ & - & - & - & M3: ${ }^{x} \mathrm{~V}$ & 64 & $1712+4539$ & 113 & 155 \\
\hline LP $71-82$ & - & - & $154^{\mathrm{f}}$ & - & 32,36 & M5 V & 32 & $1802+6415$ & 114 & 157 \\
\hline G 202-48 & - & G1 625 & 153.46 & 0.99 & 5 & M1.5 V & 39 & $1625+5418$ & 115 & 158 \\
\hline L $100-115$ & - & GJ 1128 & 153.05 & 2.41 & 17 & $\mathrm{M} 4.5 \mathrm{~V}$ & 18 & $0942-6853$ & 116 & 159 \\
\hline G $161-71$ & - & - & $153^{\mathrm{j}}$ & - & 35,72 & M5 V & 35 & $0944-1220$ & 117 & 160 \\
\hline L $1190-34$ & - & GJ 1156 & 152.9 & 3.0 & 6 & M5 V & 21 & $1218+1107$ & 118 & 161 \\
\hline $\mathrm{BD}+562966$ & LFT 1767 & Gl 892 & 152.76 & 0.29 & 5 & K3 V & 49 & $2313+5710$ & 119 & 162 \\
\hline 2MASS J11145133-2618235 & - & - & 152 & - & 1 & $\mathrm{~T} 7.5$ & 53 & $1114-2618$ & 120 & 163 \\
\hline Ross 104 & - & Gl 408 & 150.10 & 1.70 & 5 & $\mathrm{M} 2.5 \mathrm{~V}$ & 21 & $1100+2249$ & 121 & 164 \\
\hline Ross $775 \mathrm{~A}$ & - & G1 $829 \mathrm{~A}$ & 149.15 & 1.81 & 5 & $\mathrm{M} 3.5^{\mathrm{c}} \mathrm{V}$ & 39 & $2129+1738$ & 122 & 165 \\
\hline Ross 775 B & - & G1 829 B & - & - & - & M3.5: ${ }^{\mathrm{y}} \mathrm{V}$ & 1 & $2129+1738$ & 122 & 165 \\
\hline SCR J1546-5534 & - & - & 149 & - & 11 & late- $\mathrm{M}^{\mathrm{Z}} \mathrm{V}$ & 1 & $1546-5534$ & 123 & 167 \\
\hline$\xi$ Boo A & - & G1 $566 \mathrm{~A}$ & 148.98 & 0.48 & 5 & G7 V & 49 & $1451+1906$ & 124 & 168 \\
\hline$\xi$ Boo B & - & G1 566 B & - & - & - & $\mathrm{K} 4 \mathrm{~V}$ & 67 & $1451+1906$ & 124 & 168 \\
\hline Wolf 358 & - & Gl 402 & 147.92 & 3.52 & 5 & M4 V & 38 & $1050+0648$ & 125 & 170 \\
\hline G $41-14$ A & - & - & 147.66 & 1.98 & 12 & $\mathrm{M} 3.5^{\mathrm{c}} \mathrm{V}$ & 21 & $0858+0828$ & 126 & 171 \\
\hline G $41-14 \mathrm{~B}$ & - & - & - & - & - & M3.5: ${ }^{\mathrm{a} a} \mathrm{~V}$ & 1 & $0858+0828$ & 126 & 171 \\
\hline G $41-14 \mathrm{C}$ & - & - & - & - & - & M3.5: ${ }^{\mathrm{a} a} \mathrm{~V}$ & 1 & $0858+0828$ & 126 & 171 \\
\hline Ross 619 & - & G1 299 & 146.3 & 3.1 & 6 & M4 V & 21 & $0811+0846$ & 127 & 174 \\
\hline Ross 671 & - & G1 880 & 146.09 & 1.00 & 5 & $\mathrm{M} 1.5 \mathrm{~V}$ & 39 & $2256+1633$ & 128 & 175 \\
\hline LP 914-54 & LHS 3003 & - & 145.30 & 3.25 & 15 & M7 V & 66 & $1456-2809$ & 129 & 176 \\
\hline LP $771-95^{\mathrm{cc}}$ & - & - & 144.68 & 2.52 & 12 & $\mathrm{M} 2.5 \mathrm{~V}$ & 12 & $0301-1635$ & 130 & 177 \\
\hline LP 771-96 A & - & - & - & - & - & $\mathrm{M} 3^{\mathrm{d} d} \mathrm{~V}$ & 12 & $0301-1635$ & 130 & 177 \\
\hline LP 771-96 B & - & - & - & - & - & M3.5: ${ }^{\mathrm{ee}} \mathrm{V}$ & 1 & $0301-1635$ & 130 & 177 \\
\hline L $230-188$ & - & GJ 1068 & 143.42 & 1.92 & 17 & $\mathrm{M} 4.5^{\mathrm{ff} f} \mathrm{~V}$ & 44 & $0410-5336$ & 131 & 180 \\
\hline WISE J192841.35+235604.9 & - & - & 143 & - & 1 & T6 & 78 & $1928+2356$ & 132 & 181 \\
\hline L $369-44^{\mathrm{g} g}$ & LP 993-116 & - & 143 & - & 32,72 & $\mathrm{M} 4 \mathrm{~V}$ & 32 & $0245-4344$ & 133 & 182 \\
\hline L $369-45^{\mathrm{g} g}$ & LP 993-115 & - & 143 & - & 32,72 & $\mathrm{M} 5: \mathrm{h} h_{\mathrm{V}}$ & 1 & $0245-4344$ & 133 & 182 \\
\hline 2MASS J18522528-3730363 & - & - & 143 & - & 32 & $\mathrm{M} 4.5 \mathrm{~V}$ & 32 & $1852-3730$ & 134 & 184 \\
\hline G $161-7 \mathrm{~A}$ & - & - & $143^{\mathrm{k}}$ & - & 35,72 & $\mathrm{M} 5^{\mathrm{c}} \mathrm{V}$ & 35 & $0915-1035$ & 135 & 185 \\
\hline G 161-7 B & - & - & - & - & - & M5: ${ }^{n} n_{V}$ & 1 & $0915-1035$ & 135 & 185 \\
\hline $\mathrm{BD}+612068 \mathrm{~A}$ & LFT $1580 \mathrm{~A}$ & Gl $809 \mathrm{~A}$ & 141.87 & 0.64 & 5 & $\mathrm{MO}^{\mathrm{c}} \mathrm{V}$ & 39 & $2053+6209$ & 136 & 187 \\
\hline $\mathrm{BD}+612068 \mathrm{~B}$ & LFT $1580 \mathrm{~B}$ & G1 $809 \mathrm{~B}$ & - & - & - & $\mathrm{M} ?^{\mathrm{i} i} \mathrm{~V}$ & 1 & $2053+6209$ & 136 & 187 \\
\hline Ross 446 & - & G1 393 & 141.50 & 2.22 & 5 & $\mathrm{M} 2 \mathrm{~V}$ & 21 & $1028+0050$ & 137 & 189 \\
\hline WISE J071322.55-291751.9 & - & - & 141 & - & 1 & Yo & & $0713-2917$ & 138 & 190 \\
\hline WISE J000517.48+373720.5 & - & - & 141 & - & 1 & T9 & 78 & $0005+3737$ & 139 & 191 \\
\hline
\end{tabular}


Table 4-Continued

\begin{tabular}{|c|c|c|c|c|c|c|c|c|c|c|}
\hline $\begin{array}{c}\text { Discovery Name } \\
\text { (1) }\end{array}$ & $\begin{array}{l}\text { Other Name } \\
\text { (2) }\end{array}$ & $\begin{array}{c}\text { Gliese Name } \\
\text { (3) }\end{array}$ & $\begin{array}{l}\pi_{t r i g}{ }^{a} \\
\left(\operatorname{mas}^{2}\right)\end{array}$ & $\begin{array}{l}\sigma_{\pi} \\
(5)\end{array}$ & $\begin{array}{c}\pi \text { Ref. } \\
\text { (6) }\end{array}$ & $\begin{array}{l}\text { Spec. } \\
\text { Type } \\
(7)\end{array}$ & $\begin{array}{l}\text { Spec. } \\
\text { Ref. } \\
(8)\end{array}$ & $\begin{array}{c}\text { J2000 Coords. } \\
\text { (9) }\end{array}$ & $\begin{array}{l}\text { Sys. } \\
\text { Rank } \\
(10)\end{array}$ & $\begin{array}{l}\text { Indiv. } \\
\text { Rank } \\
(11)\end{array}$ \\
\hline WISEPC J121756.91+162640.2 & - & - & 141 & - & 1 & T9 & 3 & $1217+1626$ & 140 & 192 \\
\hline 2MASS J07290002-3954043 & - & - & 141 & - & 1 & $\mathrm{~T} 8 \mathrm{p}$ & 79 & $0729-3954$ & 141 & 193 \\
\hline HR 6426 A & LFT $1336 \mathrm{~A}$ & G1 $667 \mathrm{~A}$ & 139.5 & 6.3 & 6 & $\mathrm{~K} 4-\mathrm{V}$ & 50 & $1718-3459$ & 142 & 194 \\
\hline HR 6426 B & LFT 1336 B & G1 $667 \mathrm{~B}$ & - & - & - & $\mathrm{K} 4 \mathrm{~V}$ & 56 & $1718-3459$ & 142 & 194 \\
\hline HR $6426 \mathrm{C}$ & LFT 1337 & G1 $667 \mathrm{C}$ & - & - & - & M1.5 V & 44 & $1718-3459$ & 142 & 194 \\
\hline HR 753 A & LFT $217 \mathrm{~A}$ & G1 $105 \mathrm{~A}$ & 139.27 & 0.45 & 5 & K3 V & 49 & $0236+0653$ & 143 & 197 \\
\hline HR 753 B & LFT 218 & G1 $105 \mathrm{~B}$ & - & - & - & M3.5 V & 21 & $0236+0652$ & 143 & 197 \\
\hline HR $753 \mathrm{C}$ & LFT $217 \mathrm{~B}$ & G1 $105 \mathrm{C}$ & - & - & - & $M 7^{: j j} \mathrm{~V}$ & 68 & $0236+0653$ & 143 & 197 \\
\hline SCR J0740-4257 & LSR J07401-4257 & - & 139 & - & 7 & $\mathrm{M} 4.5 \mathrm{~V}$ & 7 & $0740-4257$ & 144 & 200 \\
\hline L $43-72$ & - & - & 139 & - & 14 & $\mathrm{M} 4.5 \mathrm{~V}$ & 14 & $1811-7859$ & 145 & 201 \\
\hline G $157-77$ & - & GJ 1286 & 138.3 & 3.5 & 6 & M5.5 V & 21 & $2335-0223$ & 146 & 202 \\
\hline LP $229-17^{1 l}$ & - & - & 138 & 40 & 37 & M3 V & 37 & $1834+4007$ & 147 & 203 \\
\hline LP $71-165$ & LHS 3376 & - & 137.5 & 5.3 & 6 & $\mathrm{M} 4.5 \mathrm{~V}$ & 21 & $1818+6611$ & 148 & 204 \\
\hline 2MASSW J1507476-162738 & - & - & 136.4 & 0.6 & 30 & L5 & 61 & $1507-1627$ & 149 & 205 \\
\hline WISE J004945.61+215120.0 & - & - & 135 & - & 1 & $\mathrm{~T} 8.5$ & 78 & $0049+2151$ & 150 & 206 \\
\hline L $788-34$ & LHS 3799 & - & 134.4 & 4.9 & 6 & $\mathrm{M} 4.5 \mathrm{~V}$ & 21 & $2223-1736$ & 151 & 207 \\
\hline G $203-47$ A & - & - & 134.31 & 1.99 & 5 & M3.5 $\mathrm{V}^{*}$ & 21 & $1709+4340$ & 152 & 208 \\
\hline G $203-47$ B & - & - & 134.31 & 1.99 & 5 & $\mathrm{D} ?^{\mathrm{P} p}$ & 43 & $1709+4340$ & 152 & 208 \\
\hline L $499-56$ & LHS 3746 & - & 134.29 & 1.31 & 12 & M3 V & 12 & $2202-3704$ & 153 & 210 \\
\hline Lalande 1299 & - & Gl 33 & 134.14 & 0.51 & 5 & $\mathrm{~K} 2 \mathrm{~V}$ & 49 & $0048+0516$ & 154 & 211 \\
\hline$\beta$ Нyi & - & Gl 19 & 134.07 & 0.11 & 5 & G0 $\mathrm{V}$ & 50 & $0025-7715$ & 155 & 212 \\
\hline Ross 556 & - & G1 109 & 133.16 & 2.26 & 5 & M3 $\mathrm{V}$ & 21 & $0244+2531$ & 156 & 213 \\
\hline 107 Psc & - & G1 68 & 132.76 & 0.50 & 5 & Ko V & 49 & $0142+2016$ & 157 & 214 \\
\hline G $154-44$ & - & GJ 1224 & 132.6 & 3.7 & 6 & $\mathrm{M} 4.5 \mathrm{~V}$ & 21 & $1807-1557$ & 158 & 215 \\
\hline$\mu$ Cas $\mathrm{A}$ & - & G1 $53 \mathrm{~A}$ & 132.38 & 0.82 & 5 & $\mathrm{~K} 1 \mathrm{~V}$ Fe-2 & 49 & $0108+5455$ & 159 & 216 \\
\hline$\mu$ Cas B & - & G1 $53 \mathrm{~B}$ & - & - & - & M3.5: ${ }^{\mathrm{q} q} \mathrm{~V}$ & 9 & $0108+5455$ & 159 & 216 \\
\hline LTT $9283^{\text {tt }}$ & - & Gl 879 & 131.42 & 0.62 & 5 & $\mathrm{~K} 4+\mathrm{V} \mathrm{k}$ & 50 & $2256-3133$ & 160 & 218 \\
\hline Ross 490 & - & G1 514 & 130.62 & 1.05 & 5 & M1 V & 21 & $1329+1022$ & 161 & 219 \\
\hline Vega & - & G1 721 & 130.23 & 0.36 & 5 & $\mathrm{~A} 0 \mathrm{Va}$ & 49 & $1836+3847$ & 162 & 220 \\
\hline Wolf 718 & - & Gl 673 & 129.86 & 0.73 & 5 & $\mathrm{~K} 7 \mathrm{~V}$ & 21 & $1725+0206$ & 163 & 221 \\
\hline Fomalhaut $^{\mathrm{r} r}$ & - & Gl 881 & 129.81 & 0.47 & 5 & $\mathrm{~A} 4 \mathrm{~V}$ & 50 & $2257-2937$ & 164 & 222 \\
\hline LP $881-64 \mathrm{~A}^{\circ o}$ & - & GJ $2005 \mathrm{~A}$ & 129.47 & 2.48 & 15 & M5. $5 \mathrm{~V}$ & 69 & $0024-2708$ & 165 & 223 \\
\hline LP 881-64 B & - & GJ 2005 B & - & - & - & $\mathrm{M} 8.5 \mathrm{~V}$ & 69 & $0024-2708$ & 165 & 223 \\
\hline LP $881-64 \mathrm{C}$ & - & GJ $2005 \mathrm{C}$ & - & - & - & M9 V & 69 & $0024-2708$ & 165 & 223 \\
\hline L $991-14$ & - & Gl 701 & 128.89 & 1.43 & 5 & Mo V & 21 & $1805-0301$ & 166 & 226 \\
\hline G $109-35$ & - & GJ 1093 & 128.8 & 3.5 & 6 & M5 V & 21 & $0659+1920$ & 167 & 227 \\
\hline Innes (unnumbered) ${ }^{g}$ & - & Gl 480.1 & 128.52 & 3.90 & 5 & M3 V & 44 & $1240-4333$ & 168 & 228 \\
\hline WISEP J060738.65+242953.4 & - & - & 128.2 & - & 71 & L9 & 1 & $0607+2429$ & 169 & 229 \\
\hline 2MASS J03480772-602227 & - & - & 128 & - & 1 & $\mathrm{~T} 7$ & 53 & $0348-6022$ & 170 & 230 \\
\hline p Eri A & - & Gl $66 \mathrm{~A}$ & 127.84 & 2.19 & 5 & $\mathrm{~K} 2 \mathrm{~V}$ & 50 & $0139-5611$ & 171 & 231 \\
\hline p Eri B & - & Gl $66 \mathrm{~B}$ & & - & - & $\mathrm{K} 2 \mathrm{~V}$ & 50 & $0139-5611$ & 171 & 231 \\
\hline $\mathrm{BD}-032870$ & LFT 3734 & G1 382 & 127.08 & 1.90 & 5 & $\mathrm{M} 2 \mathrm{~V}$ & 38 & $1012-0344$ & 172 & 233 \\
\hline L $97-12$ & & Gl 293 & 126.25 & 1.34 & 8 & DQ9 & 52 & $0753-6747$ & 173 & 234 \\
\hline L $1813-21$ & LHS 1805 & - & 126.1 & 3.3 & 75 & M3.5 V & 21 & $0601+5935$ & 174 & 235 \\
\hline LP $876-10$ & - & - & $126^{1}$ & - & 35,70 & M4 V & 70 & $2248-2422$ & 175 & 236 \\
\hline L $674-15$ & - & Gl 300 & 125.60 & 0.97 & 12 & $\mathrm{M} 3.5 \mathrm{~V}$ & 21 & $0812-2133$ & 176 & 237 \\
\hline $\mathrm{AC}+656955$ & LFT 1552 & G1 793 & 125.07 & 1.08 & 5 & $\mathrm{M} 2.5 \mathrm{~V}$ & 44 & $2030+6526$ & 177 & 238 \\
\hline G $99-47$ & - & GJ 1087 & 125.0 & 3.6 & 6 & DAP9 & 52 & $0556+0521$ & 178 & 239 \\
\hline
\end{tabular}


Table 4-Continued

\begin{tabular}{|c|c|c|c|c|c|c|c|c|c|c|}
\hline $\begin{array}{c}\text { Discovery Name } \\
\text { (1) }\end{array}$ & $\begin{array}{c}\text { Other Name } \\
\text { (2) }\end{array}$ & $\begin{array}{c}\text { Gliese Name } \\
\text { (3) }\end{array}$ & $\begin{array}{l}\pi_{\text {trig }}{ }^{\mathrm{a}} \\
(\mathrm{mas}) \\
(4)\end{array}$ & $\begin{array}{c}\sigma_{\pi} \\
(\mathrm{mas}) \\
(5)\end{array}$ & $\begin{array}{c}\pi \text { Ref. } \\
\text { (6) }\end{array}$ & $\begin{array}{l}\text { Spec. } \\
\text { Type } \\
(7)\end{array}$ & $\begin{array}{l}\text { Spec. } \\
\text { Ref. } \\
(8)\end{array}$ & $\begin{array}{c}\text { J2000 Coords. } \\
\text { (9) }\end{array}$ & $\begin{array}{l}\text { Sys. } \\
\text { Rank } \\
(10)\end{array}$ & $\begin{array}{c}\text { Indiv. } \\
\text { Rank } \\
(11)\end{array}$ \\
\hline LP $467-16$ & - & - & 125 & - & 32,73 & M5 V & 3 & $0111+1526$ & 179 & 240 \\
\hline 2MASS J12140866-2345172 & - & - & 125 & - & 32 & $\mathrm{M} 4.5 \mathrm{~V}$ & 32 & $1214-2345$ & 180 & 241 \\
\hline 2MASS J19513587-3510375 & - & - & 125 & - & 32 & M4 V & 32 & $1951-3510$ & 181 & 242 \\
\hline SCR J0838-5855 & - & - & 125 & - & 7 & M6 V & 7 & $0838-5855$ & 182 & 243 \\
\hline
\end{tabular}

Note. - Code for references: (1) this paper, (2) Marsh et al. (in prep.), (3) Kirkpatrick et al. (2011), (4) Cushing et al. (2011), (5) van Leeuwen, (2007), (6) van Altena et al. (1995), (7) Winters et al. (2011), (8) Subasavage et al. (2009), (9) Drummond et al. (1995), (10) Riedel et al. (2011), (11) Boyd et al. (2011), (12) Henry et al. (2006), (13) Costa et al. (2006), (14) Scholz et al. (2005a), (15) Costa et al. (2005), (16) Benedict et al. (1999), (17) Jao et al. (2005), (18) Henry et al. (2002), (19) Hawlev et al. (1996), (20) Henry et al. (2004), (21) Henry et al. (1994), (22) Lucas et al. (2010), (23) Artigau et al. (2010), (24) Hershey \& Taff (1998), (25) Ducourant et al. (1998), (26) Schilbach et al. 2009), (27) Tinney (1996), (28) Burgasser et al. (2008), (29) Vrba et al. (2004), (30) Dahn et al. (2002), (31) Reid et al. (2003), (32) Riaz et al. (2006), (33) Scholz (2010), (34) Artigau et al. (2006), (35) Scholz et al. (2005b), (36) Reid et al. (2007), (37) Jenkins et al. (2009), (38) Kirkpatrick et al., (1991), (39) Kirkpatrick (1992), (40) Henry et al. (1992), (41) Boeshaar \& Tyson (1985), (42) Kirkpatrick \& McCarthy (1994), (43) Delfosse et al. (1999), (44) Hawley et al. (1996), (45) Delfosse et al. (1999), (46) Torres et al. (1999), (47) McCarthy et al. (1988), (48) Kirkpatrick et al. (1999), (49) Gray et al. (2003), (50) Gray et al. (2006), (51) Garrison (1994), (52) McCook \& Sion (2006), (53) Burgasser et al. (2006a), (54) Kasper et al. (2007), (55) Kirkpatrick et al. (2008), (56) Bidelman (1985), (57) Forveille et al. (1999), (58) Grav et al. (2001), (59) Irwin et al. (1996), (60) Tomkin \& Pettersen (1986), (61) Kirkpatrick et al. (2000), (62) Burgasser et al. (2010), (63) Kirkpatrick et al. (2010), (64) Henry \& McCarthy (1993), (65) Reid et al. (1995), (66) Kirkpatrick et al. (1995), (67) Wilson \& Vainu Bappu (195), (68) Golimowski et al. (2000), (69) Reiners et al. (2007),

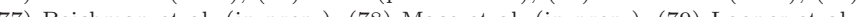
77) Beichman et al. (in prep.), (78) Mace et al, (in prep.), (79) Looper et al. (2007).

${ }^{a}$ Unless otherwise marked, the parallax for the primary (or for the combined system) is used for all components of the system.

${ }^{\mathrm{b}}$ Proxima Centauri is the third member of the $\alpha$ Centauri system. The error bar quoted here for the parallax is the updated one used in Jao et al. (2005).

${ }^{c}$ The spectral type is a joint type including all objects in the system.

d These spectral types are estimates based on the $M_{V}$ values for the B and C components as measured by Delfosse et al. (1999) and translated into spectral type using Table 3 of Kirkpatrick \& McCarthy (1994)

${ }^{e}$ This object is sometimes referred to as van Maanen 2, although in the original discovery paper van Maanen 1917) it is neither named or numbered.

${ }^{\mathrm{f}}$ We estimate a distance by averaging the $7.1 \mathrm{pc}$ estimate of Reid et al. (2007) and the 6 pc estimate of Riaz et al. (2006).

"According to Luvten (1979), Innes discovered this object. However, Innes didn't number his discoveries, so the discovery name is noted simply as "Innes (unnumbered)".

${ }^{\mathrm{h}}$ This is a composite type for G 208-44 AB (GJ $1245 \mathrm{AC}$ ).

McCarthy et al. (1988) give delta(V) $\approx 2.0$ mag for the G 208-44 AB pair, so we estimate the spectral type of G $208-44$ B to be approx one subclass later than G 208-44 A (assumed to have a type identical to the composite spectral type of M5.5 V).

${ }^{\mathrm{j}}$ We estimate a distance by averaging the $6.9 \mathrm{pc}$ estimate of Scholz et al. (2005b) and the $6.2 \mathrm{pc}$ estimate of Reid et al. (2002).

${ }^{k}$ We estimate a distance by averaging the 7.3 pc estimate of [Scholz et al. (2005b) and the 6.7 pc estimate of Reid et al. (2002).

We estimate a distance by averaging the $7.2 \mathrm{pc}$ estimate of Reid et al. (2004) and the $8.7 \mathrm{pc}$ estimate of Scholz et al. (2005b).

${ }^{m}$ This spectral type is a composite type for the Lalande $27173 \mathrm{BC}$ pair.

${ }^{\mathrm{n}}$ This spectral type is estimated from the absolute $J, H, K$ values measured by Forveille et al. (1999) and the absolute magnitudes versus spectral type relation given in Table 3 of Kirkpatrick \& McCarthy (1994)

The parallax for $36 \mathrm{Oph} \mathrm{A}$ is assumed for $36 \mathrm{Oph} \mathrm{C}$; an independent Hipparcos parallax for $36 \mathrm{Oph} \mathrm{C}$ is 167.49 \pm 0.60 mas van Leeuwen 2007).

P 2MASS J18450079-1409036 and 2MASS J18450097-1409053 appear to be a common proper motion pair based on Digitized Sky Survey $R$-band images taken in 1951 and again in 1988 (see http://irsa.ipac.caltech.edu/applications/FinderChart/). 
(Hawlev et al. (1997) say this is an M3 III but Luvten (1979) lists this pair as a common proper motion binary so the giant luminosity classification must be an error.

[Reid et al. (2004) state that they believe this parallax measurement may be in error because the result suggests a location on the H-R diagram significantly below the main sequence despite the fact that this M dwarf's spectrum shows no evidence of the low metallicity expected for such a location.

${ }^{\mathrm{s}}$ Identification taken from van de Kamp (1930).

${ }^{t}$ This spectral type is the composite type for Wolf $630 \mathrm{ABaBb}$

${ }^{4}$ The spectral type is estimated from the measured mass of this component (Ségransan et al. 2000) and a mapping of mass into spectral type using other objects in this table and their masses as measured in Henry \& McCarthy (1993)

Costa et al. (2005) measure an independent parallax of 155.43+/-1.33 mas for van Biesbroeck 8.

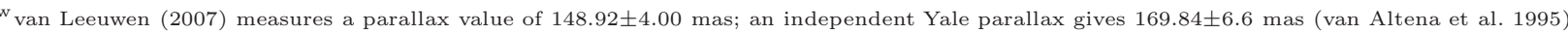

x This spectral type is based on delta $J, H, K$ values measured by Henry \& McCarthy (1993) and using the absolute magnitude versus spectral types tabulate in Table 3 of Kirkpatrick \& McCarthy (1994)

Given that the mass ratio is $\sim 1$ (Delfosse et al. 1999), we assume equal spectral types for both components.

${ }^{\mathrm{z}} \mathrm{SCR}$ J1546-5534 has $J-K s=1.10$ mag from 2MASS; WISE photometry is corrupted by a brighter source immediately to the northwest (the Galactic latitude of this source is -0.8 deg) so we can only say that $J$-W2 $<3.65$ and $H$-W $2<2.99$. The overall photometry from both 2MASS and WISE is consistent with a late-M dwarf.

${ }^{a} a$ Given that Delfosse et al. (1999) find nearly equal masses for components A and B and that component $\mathrm{C}$ is $\sim 0.5$ mag fainter than the joint AB pair in adaptive optics imaging, we assume all components are roughly the same spectral type.

${ }^{b b}$ Identification taken from Hertzsprung (1922); also referred to as Cordoba $5 \mathrm{~h} 243$ van Maanen 1943).

${ }^{{ }^{c} c}$ The object LP 771-95 shares common proper motion with LP 771-96 AB. The parallax measured for LP 771-95 is assumed for LP 771-96 AB.

$\mathrm{d} d$ This is the composite spectral type for LP 771-96 AB

${ }^{e}$ This spectral type is estimated from the delta $V, R, I$ values measured in Henry et al. (2006), the measured composite type for LP 771-96 AB, and spectral type versus absolute magnitude relations given in Table 3 of Kirkpatrick \& McCarthv (1994).

$\mathrm{f} f$ Rodgers \& Eggen (1974) note peculiarities in the blue spectrum of this object.

${ }^{g}$ These two objects share common proper motion.

hh Kuiper (1943) types this object one spectral subclass later than L 369-44; as Kuiper was probably typing on the Mt. Wilson system, we assign this object a type one subclass later than the type assigned for L $369-44$ by Riaz et al. (2006).

${ }^{i i} \mathrm{G}$ 231-19 is noted as a spectroscopic binary by Herbst \& Layden (1987) (see also Heintz 1981). No other info is given, so an M spectral type is assumed for the secondary.

${ }^{j}$ The spectral type is an estimate based on measured broad-band colors.

${ }^{1 l}$ The object LP 229-17 lies near on the sky and in distance to Vega.

$\mathrm{nn}$ Montagnier et al. (2006) show that this is a near equal-magnitude binary, so we estimate a spectral type identical to the joint spectrum.

${ }^{\circ} \mathrm{GJ} 2005 \mathrm{C}$, a fourth component reported elsewhere, may not exist according to Seifahrt et al. (2008).

${ }^{p}$ This object is believed to be a white dwarf.

${ }^{q} q$ This spectral type is an estimate based on the measured absolute $\mathrm{V}$ magnitude.

${ }^{r}$ Fomalhaut has common proper motion with LTT 9283 (Gl 879).

${ }^{t} t$ The object LTT 9283 has common proper motion with Fomalhaut but may lie slightly closer to the Sun than Fomalhaut itself.

${ }^{u} u$ Marsh et al. (in prep.) report a parallax of $0^{\prime \prime} 087 \pm 0$. ' 054 for this object, whereas Kirkpatrick et al. (2011) report $00^{\prime \prime} 351 \pm 0$.' 108 . Given the discrepancies in this measurements, we instead use our spectrophotometric distance estimate from Table 8 
Table 5. Objects Once Listed as Falling Within 8 pc But Now Believed To Lie More Distant

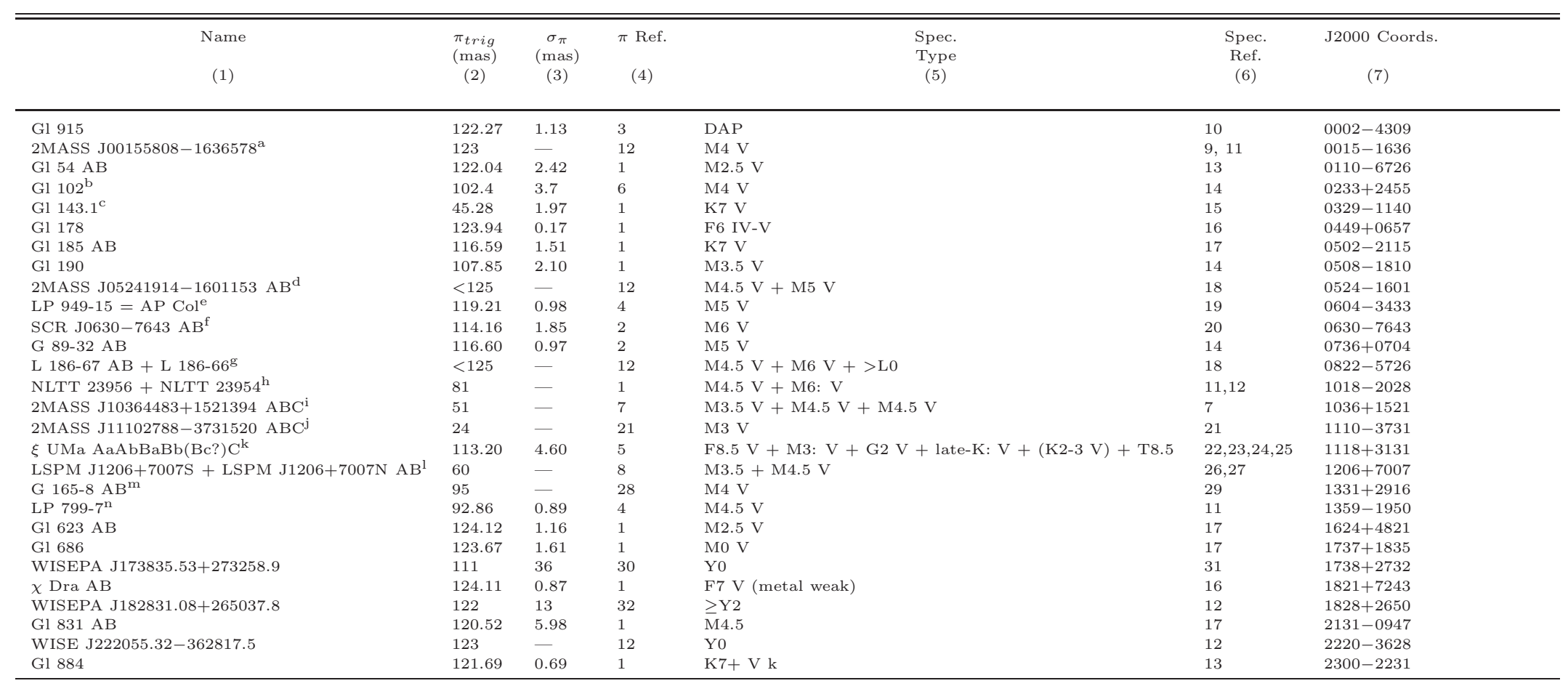

Note. - Code for references: (1) van Leeuwen (2007), (2) Henry et al. (2006), (3) Subasavage et al. (2009), (4) Riedel et al. (2011), (5) Perryman et al. (1997), (6) van Altena et al. (1995), (7) Daemgen et al. (2007), (8) Shkolnik et al. (2010), (9) Reid et al. (2007), (10) Koester et al. (2009), (11) Riaz et al. (2006), (12) this paper, (13) Grav et al. (2006), (14) Reid et al. (1995), (15) Hawley et al. 1997), (16) Grav et al. (2001), (17) Henry et al. (1994), (18) Bergfors et al. (2010), (19) Scholz et al. 2005a), (20) Henry et al. (2004), (21) Zuckerman \& Song (2004), (22) Kenan e Meid et al. 2004), (30) Marsh et al. (in prep.), (31) Cushing et al, (2011) (32) Beict (in prep.).

Reid et al. (2007) predicted a distance of $7.3 \mathrm{pc}$ and Riaz et al. (2006) predicted 9 pc. We list the average of these two as the distance estimate.

Riaz et al. (2006) predicted a distance of 8 pc.

(Riaz et al. (2006) predicted a distance of 4 pc based on what appears to be an erroneous measurement (M4.5 V) of this star's spectral type.

Riaz et al. (2006) predicted a distance of 8 pc assuming this is a single star, but Bergfors et al. (2010) have shown this to be a near equal-mag double so the distance must be greater.

Scholz et al. 2005a) predicted a distance of $6.1 \mathrm{pc}$.

Winters et al. (2011) predicted a distance of 5.5 pc.

$\sqrt[8]{\text { Riaz et al. (2006) }}$ predicted a distance of 8 pc to L 186-67 (LHS 2005) assuming it is single. Bergfors et al. (2010) find that L 186-67 is a close double (M4.5 V $+>$ L0) with a magnitude difference of $4.5 \mathrm{mag}$ at $z$ band. We assume that the binarity is enough to push this object outside of our 8 pc limit, but parallactic verification is warranted. Note that there is a common 
proper motion, third component (M6 V) named L 186-66 (LHS 2004).

Riaz et al. (2006) predict a distance of 8 pc. However, their spectral type of M4.5 V for NLTT 23956 along with its 2MASS values of $J=9.00, H=8.42$, and $K_{s}=8.15$ mag suggest, using Table 3 of Kirkpatrick \& McCarthy (1994), a distance of 12.4, 12.3, and $12.2 \mathrm{pc}$ in each band, respectively. Our estimate of $12.3 \mathrm{pc}$ is assumed instead. We estimate a spectral type of M6 V for the fainter common proper motion companion, NLTT 23954, based on the fact that is it 1.5 mag fainter than NLTT 23956 in all 2 MASS bands.

Reid et al. (2007) predict a distance of $7.1 \mathrm{pc}$ assuming it is single. Daemgen et al. (2007), however, have shown it to be a triple system.

This object is also known as TWA 3 ABC, a member of the TW Hydrae Association, and believed to lie at a distance of 42 pc (Zuckerman \& Song 2004), not 5 pc away as estimated by Riaz et al. (2006).

${ }^{k}$ An earlier parallax measurement, $127 \pm 6$ mas (Jenkins 1952), places this just inside the 8-pc volume. This is a remarkable quintuple or sextuple system. The speckle companion $\xi$ UMa Bc Mason et al. 1995) awaits independent verification, and a widely separated T8.5 companion ( $\xi$ UMa C) has recently been announced by Wright et al. (2012).

Reid et al. (2007) predicted a distance of 5.1 pc for LSPM J1206+7007S. Shkolnik et al. (2010) find LSPM J1206+7007N to be a double-lined spectroscopic binary

meid et al. (2004) predicted a distance of $7.9 \mathrm{pc}$ assuming the star is single, but Beuzit et al. (2004) find it to be binary and adjust the distance estimate accordingly.

niaz et al. (2006) predicted a distance of $7 \mathrm{pc}$ 
Table 6. Late-T and Y Dwarfs with Measured Trigonometric Parallaxes

\begin{tabular}{|c|c|c|c|c|c|c|c|c|c|}
\hline $\begin{array}{c}\text { Discovery Name } \\
\text { (1) }\end{array}$ & $\begin{array}{c}\text { Disc. Ref. } \\
\text { (2) }\end{array}$ & $\begin{array}{c}\text { Spec. Type } \\
\text { (3) }\end{array}$ & $\begin{array}{c}\text { Type Ref. } \\
\text { (4) }\end{array}$ & $\begin{array}{l}\text { W1 } \\
(\mathrm{mag}) \\
(5)\end{array}$ & $\begin{array}{l}\text { W2 } \\
(\mathrm{mag}) \\
(6)\end{array}$ & $\begin{array}{c}\mathrm{H} \\
(\mathrm{mag}) \\
(7)\end{array}$ & $\begin{array}{l}\text { H Ref. } \\
\text { (8) }\end{array}$ & $\begin{array}{l}\pi_{t r i g} \\
(\operatorname{arcsec}) \\
\quad(9)\end{array}$ & $\begin{array}{c}\pi_{\text {trig }} \text { Ref. } \\
\text { (10) }\end{array}$ \\
\hline SDSS J000013.54+255418.6 & 1 & $\mathrm{~T} 4.5$ & 52 & - & - & $14.73 \pm 0.07$ & 38 & $0.0708 \pm 0.0019$ & 39 \\
\hline ULAS J003402.77-005206.7 & 2 & $\mathrm{~T} 8.5$ & 32 & $17.47 \pm 0.29$ & $14.50 \pm 0.08$ & $18.49 \pm 0.04$ & 2 & $0.0780 \pm 0.0036$ & 40 \\
\hline HD 3651B (J0039+2115) & 3 & $\mathrm{~T} 7.5$ & 3 & & - & $16.68 \pm 0.04$ & 3 & $0.0904 \pm 0.0003$ & 41 \\
\hline CFBDS J005910.90-011401.3 & 4 & $\mathrm{~T} 8.5$ & 32 & $17.07 \pm 0.15$ & $13.68 \pm 0.04$ & $18.27 \pm 0.05$ & 4 & $0.1011 \pm 0.0025$ & 39 \\
\hline SDSS J020742.48+000056.2 & 5 & $\mathrm{~T} 4.5$ & 52 & $16.39 \pm 0.08$ & $15.07 \pm 0.08$ & - & - & $0.0293 \pm 0.0040$ & 40 \\
\hline 2MASSI J0243137-245329 & 6 & $\mathrm{~T} 6$ & 52 & $14.67 \pm 0.03$ & $12.92 \pm 0.03$ & $15.14 \pm 0.11$ & 38 & $0.0936 \pm 0.0036$ & 42 \\
\hline WISEPA J025409.45+022359.1 & 7,8 & $\mathrm{~T} 8$ & 7 & $15.76 \pm 0.05$ & $12.74 \pm 0.03$ & $16.30 \pm 0.01$ & 51 & $0.166 \pm 0.026$ & 43 \\
\hline WISE J035000.32-565830.2 & 51 & Y1 & 51 & $>18.90$ & $14.73 \pm 0.06$ & $>21.5$ & 51 & $0.238 \pm 0.038$ & 43 \\
\hline WISEP J041022.71+150248.5 & 32 & Y0 & 32 & $>18.33$ & $14.18 \pm 0.06$ & $19.05 \pm 0.09$ & 51 & $0.164 \pm 0.024$ & 43 \\
\hline 2MASSI J0415195-093506 & 6 & $\mathrm{~T} 8$ & 52 & $15.11 \pm 0.04$ & $12.26 \pm 0.03$ & $15.54 \pm 0.11$ & 38 & $0.1773 \pm 0.0022$ & 39 \\
\hline WISE J053516.80-750024.9 & 51 & $\geq \mathrm{Y} 1:$ & 51 & $>18.92$ & $15.06 \pm 0.07$ & $>21.6$ & 51 & $0.170 \pm 0.044$ & 43 \\
\hline 2MASS J05591914-1404488 & 9 & $\mathrm{~T} 4.5$ & 52 & $13.39 \pm 0.03$ & $11.90 \pm 0.02$ & $13.68 \pm 0.04$ & 38 & $0.0966 \pm 0.0010$ & 39 \\
\hline G1 229B (J0610-2152) & 10 & T7p & 52 & - & - & $14.35 \pm 0.05$ & 12 & $0.1738 \pm 0.0010$ & 41 \\
\hline UGPS J072227.51-054031.2 & 11 & T9 & 32 & $15.19 \pm 0.05$ & $12.21 \pm 0.03$ & $16.90 \pm 0.02$ & 11 & $0.246 \pm 0.033$ & 11 \\
\hline 2MASSI J0727182+171001 & 6 & $\mathrm{~T} 7$ & 52 & $15.24 \pm 0.05$ & $12.96 \pm 0.03$ & $15.76 \pm 0.17$ & 38 & $0.1101 \pm 0.0023$ & 42 \\
\hline HIP 38939B (J0758-2539) & 13 & $\mathrm{~T} 4.5$ & 13 & $15.92 \pm 0.08$ & $13.96 \pm 0.05$ & $15.80 \pm 0.12$ & 38 & $0.0540 \pm 0.0011$ & 41 \\
\hline DENIS J081730.0-615520 & 14 & $\mathrm{~T} 6$ & 14 & $12.96 \pm 0.02$ & $11.24 \pm 0.02$ & $13.53 \pm 0.03$ & 38 & $0.203 \pm 0.013$ & 14 \\
\hline ULAS J082707.67-020408.2 & 15 & $\mathrm{~T} 5.5$ & 15 & $>17.77$ & $15.26 \pm 0.12$ & $17.44 \pm 0.05$ & 15 & $0.0260 \pm 0.0031$ & 40 \\
\hline ULAS J090116.23-030635.0 & 15 & $\mathrm{~T} 7.5$ & 15 & $17.77 \pm 0.31$ & $14.60 \pm 0.07$ & $18.46 \pm 0.13$ & 15 & $0.0626 \pm 0.0026$ & 40 \\
\hline 2MASSI J0937347+293142 & 6 & $\mathrm{~T} 6 \mathrm{p}$ & 52 & $14.07 \pm 0.03$ & $11.66 \pm 0.02$ & $14.70 \pm 0.07$ & 38 & $0.1634 \pm 0.0018$ & 44 \\
\hline 2MASS J09393548-2448279 & 16 & T8 & 52 & $15.03 \pm 0.04$ & $11.64 \pm 0.02$ & $15.80 \pm 0.15$ & 38 & $0.1873 \pm 0.0046$ & 45 \\
\hline ULAS J094806.06+064805.0 & 15 & $\mathrm{~T} 7$ & 15 & - & - & $19.46 \pm 0.22$ & 15 & $0.0272 \pm 0.0042$ & 40 \\
\hline ULAS J101821.78+072547.1 & 15 & T5 & 15 & $17.78 \pm 0.28$ & $16.21 \pm 0.27$ & $17.87 \pm 0.07$ & 15 & $0.0250 \pm 0.0020$ & 40 \\
\hline SDSS J102109.69-030420.1 B & 17 & $\mathrm{~T} 5$ & 39 & - & - & $16.59 \pm 0.04$ & 39 & $0.0307 \pm 0.0013$ & 39 \\
\hline 2MASSI J1047538+212423 & 18 & T6.5 & 52 & $15.43 \pm 0.04$ & $12.97 \pm 0.03$ & $15.80 \pm 0.12$ & 38 & $0.0947 \pm 0.0038$ & 42 \\
\hline SDSSp J111010.01+011613.1 & 5 & T5.5 & 52 & $15.53 \pm 0.05$ & $13.92 \pm 0.05$ & $15.92 \pm 0.14$ & 38 & $0.0521 \pm 0.0012$ & 39 \\
\hline$\xi$ UMa C (J1118+3125) & 19 & $\mathrm{~T} 8.5$ & 19 & $16.16 \pm 0.07$ & $13.31 \pm 0.03$ & $18.15 \pm 0.06$ & 19 & $0.1132 \pm 0.0046$ & 57 \\
\hline ULAS J115038.79+094942.9 & 22 & T6.5p & 22 & - & - & $19.23 \pm 0.06$ & 22 & $0.0168 \pm 0.0075$ & 40 \\
\hline 2MASS $12095613-1004008$ B & 17 & T6.5: & 39 & - & - & $18.12 \pm 0.28$ & 39 & $0.0307 \pm 0.0013$ & 39 \\
\hline 2MASSI J1217110-031113 & 18 & $\mathrm{~T} 7.5$ & 52 & $15.29 \pm 0.05$ & $13.20 \pm 0.04$ & $15.75 \pm 0.12$ & 38 & $0.0908 \pm 0.0022$ & 47 \\
\hline 2MASS J12255432-2739466 A & 18 & $\mathrm{~T} 5.5$ & 39 & - & - & $15.42 \pm 0.03$ & 39 & $0.0751 \pm 0.0025$ & 47 \\
\hline 2MASS J12255432-2739466 B & 20 & T8 & 39 & - & - & $16.91 \pm 0.03$ & 39 & $0.0751 \pm 0.0025$ & 47 \\
\hline 2MASS J12373919+6526148 & 18 & $\mathrm{~T} 6.5$ & 52 & $15.48 \pm 0.05$ & $12.95 \pm 0.03$ & $15.74 \pm 0.15$ & 38 & $0.0961 \pm 0.0048$ & 42 \\
\hline Ross $458 \mathrm{C}(\mathrm{J} 1300+1221)$ & 21 & $\mathrm{~T} 8$ & 32 & $16.01 \pm 0.07$ & $13.74 \pm 0.04$ & $17.01 \pm 0.04$ & 21 & $0.0855 \pm 0.0015$ & 41 \\
\hline ULAS J131508.42+082627.4 & 22 & $\mathrm{~T} 7.5$ & 22 & - & - & $19.50 \pm 0.10$ & 22 & $0.0428 \pm 0.0077$ & 40 \\
\hline ULAS J133553.45+113005.2 & 23 & $\mathrm{~T} 8.5$ & 32 & $16.88 \pm 0.13$ & $13.87 \pm 0.04$ & $18.25 \pm 0.01$ & 23 & $0.0967 \pm 0.0032$ & 40 \\
\hline SDSSp J134646.45-003150.4 & 24 & $\mathrm{~T} 6.5$ & 52 & $15.49 \pm 0.05$ & $13.57 \pm 0.03$ & $15.46 \pm 0.12$ & 38 & $0.0683 \pm 0.0023$ & 47 \\
\hline WISEPC J140518.40+553421.5 & 32 & Yo & 32 & $>18.82$ & $14.10 \pm 0.04$ & $>20.5$ & 51 & $0.207 \pm 0.039$ & 43 \\
\hline ULAS J141623.94+134836.3 & 25 & $\mathrm{~T} 7.5$ & 53 & $16.12 \pm 0.20$ & $12.79 \pm 0.04$ & $17.58 \pm 0.03$ & 25 & $0.1099 \pm 0.0018$ & 39 \\
\hline G1 570D (J1457-2122) & 26 & $\mathrm{~T} 7.5$ & 52 & $14.82 \pm 0.03$ & $12.11 \pm 0.02$ & $15.27 \pm 0.09$ & 38 & $0.1712 \pm 0.0009$ & 41 \\
\hline CFBDSIR J145829+101343 A & 55 & $\mathrm{~T} 8.5^{\mathrm{d}}$ & 51 & - & - & $20.12 \pm 0.10$ & 56 & $0.0340 \pm 0.0026$ & 39 \\
\hline CFBDSIR J145829+101343 B & 56 & $\mathrm{~T} 9.5^{\mathrm{d}}$ & 51 & - & - & $22.51 \pm 0.16$ & 56 & $0.0340 \pm 0.0026$ & 39 \\
\hline 2MASS J15031961+2525196 & 27 & T5 & 52 & $13.51 \pm 0.02$ & $11.72 \pm 0.02$ & $13.86 \pm 0.03$ & 38 & $0.1572 \pm 0.0022$ & 39 \\
\hline SDSS J150411.63+102718.4 & 28 & $\mathrm{~T} 7$ & 28 & $16.39 \pm 0.07$ & $14.06 \pm 0.04$ & - & - & $0.0461 \pm 0.0015$ & 39 \\
\hline HIP 73786 B (1504+0538) & 54 & T6:p & 54 & $16.48 \pm 0.08$ & $14.23 \pm 0.04$ & $17.05 \pm 0.04$ & 54 & $0.0538 \pm 0.0028$ & 41 \\
\hline WISEPC J150649.97+702736.0 & 7 & $\mathrm{~T} 6$ & 7 & $13.39 \pm 0.02$ & $11.27 \pm 0.02$ & $13.91 \pm 0.04$ & 7 & $0.193 \pm 0.026$ & 43 \\
\hline SDSS J153417.05+161546.1 B & 29 & $\mathrm{~T} 5.5$ & 39 & - & - & $17.53 \pm 0.04$ & 39 & $0.0249 \pm 0.0011$ & 39 \\
\hline 2MASSI J1534498-295227 A & 6 & $\mathrm{~T} 4.5$ & 39 & - & - & $15.36 \pm 0.03$ & 39 & $0.0624 \pm 0.0013^{\mathrm{c}}$ & 39 \\
\hline
\end{tabular}


Table 6-Continued

\begin{tabular}{|c|c|c|c|c|c|c|c|c|c|}
\hline $\begin{array}{c}\text { Discovery Name } \\
\text { (1) }\end{array}$ & $\begin{array}{l}\text { Disc. Ref. } \\
\text { (2) }\end{array}$ & $\begin{array}{l}\text { Spec. Type } \\
\text { (3) }\end{array}$ & $\begin{array}{c}\text { Type Ref. } \\
\text { (4) }\end{array}$ & $\begin{array}{l}\mathrm{W} 1 \\
(\mathrm{mag}) \\
(5)\end{array}$ & $\begin{array}{l}\mathrm{W} 2 \\
(\mathrm{mag}) \\
(6)\end{array}$ & $\begin{array}{c}\mathrm{H} \\
(\mathrm{mag}) \\
(7)\end{array}$ & $\begin{array}{l}\text { H Ref. } \\
\text { (8) }\end{array}$ & $\begin{array}{l}\pi_{\text {trig }} \\
(\operatorname{arcsec}) \\
\quad(9)\end{array}$ & $\begin{array}{c}\pi_{\text {trig }} \text { Ref. } \\
\text { (10) }\end{array}$ \\
\hline 2MASSI J1534498-295227 B & 20 & T5 & 39 & - & - & $15.64 \pm 0.03$ & 39 & $0.0624 \pm 0.0013^{\mathrm{c}}$ & 39 \\
\hline 2MASSI J1546291-332511 & 6 & $\mathrm{~T} 5.5$ & 52 & $15.30 \pm 0.05$ & $13.45 \pm 0.04$ & $15.45 \pm 0.09$ & 38 & $0.0880 \pm 0.0019$ & 47 \\
\hline 2MASSI J1553022+153236 A & 6 & T6.5 & 39 & - & - & $16.34 \pm 0.03$ & 39 & $0.0751 \pm 0.0009$ & 39 \\
\hline 2MASSI J1553022+153236 B & 17 & $\mathrm{~T} 7.5$ & 39 & - & - & $16.72 \pm 0.03$ & 39 & $0.0751 \pm 0.0009$ & 39 \\
\hline SDSSp J162414.37+002915.6 & 30 & T6 & 52 & $15.12 \pm 0.04$ & $13.09 \pm 0.03$ & $15.52 \pm 0.10$ & 38 & $0.0909 \pm 0.0012$ & 47 \\
\hline SDSS J162838.77+230821.1 & 28 & $\mathrm{~T} 7$ & 28 & $16.43 \pm 0.09$ & $13.96 \pm 0.04$ & $16.11 \pm 0.15$ & 38 & $0.0751 \pm 0.0009$ & 39 \\
\hline WISE J173835.53+273259.0 & 32 & Y0 & 32 & $>18.41$ & $14.55 \pm 0.06$ & $20.39 \pm 0.33$ & 51 & $0.111 \pm 0.036$ & 43 \\
\hline WISEPA J174124.26+255319.5 & $31,7,8$ & T9 & 7 & $15.38 \pm 0.05$ & $12.33 \pm 0.03$ & $16.63 \pm 0.03$ & 7 & $0.176 \pm 0.022$ & 43 \\
\hline SDSS J175805.46+463311.9 & 1 & $\mathrm{~T} 6.5$ & 52 & $15.68 \pm 0.04$ & $13.82 \pm 0.03$ & $16.25 \pm 0.22$ & 38 & $0.0710 \pm 0.0019$ & 41 \\
\hline WISEPA J182831.08+265037.8 & 32 & $\geq \mathrm{Y} 2$ & 51 & $>18.47$ & $14.39 \pm 0.06$ & $22.85 \pm 0.24$ & 7,32 & $0.122 \pm 0.013$ & 50 \\
\hline SCR $1845-6357$ B & 33 & $\mathrm{~T} 6$ & 34 & - & - & $13.19 \pm 0.03$ & 34 & $0.2595 \pm 0.0011$ & 48 \\
\hline Wolf 940 B (J2146-0010) & 35 & $\mathrm{~T} 8.5$ & 32 & $16.72 \pm 0.12$ & $14.24 \pm 0.05$ & $18.77 \pm 0.03$ & 35 & $0.0835 \pm 0.0039$ & 49 \\
\hline eps Indi $\mathrm{Bb}(\mathrm{J} 2204-5646)$ & 36,37 & $\mathrm{~T} 6$ & 52 & - & - & $13.27 \pm 0.10^{\mathrm{a}}$ & 37 & $0.2761 \pm 0.0003$ & 41 \\
\hline 2MASSI J2356547-155310 & 6 & $\mathrm{~T} 5.5$ & 52 & $15.58 \pm 0.06$ & $13.71 \pm 0.04$ & $15.63 \pm 0.10$ & 38 & $0.0690 \pm 0.0034$ & 42 \\
\hline
\end{tabular}

Note. - References: (1) Knapp et al. (2004), (2) Warren et al. (2007), (3) Luhman et al. (2007), (4) Delorme et al. (2008), (5) Geballe et al. (2002), (6) Burgasser et al. (2002), (7) Kirkpatrick et al. (2011), (8) Scholz et al. (2011), (9) Burgasser et al. (2000a), (10) Nakajima et al. (1995), (11) Lucas et al. (2010), (12) Leggett et al. (1999), (13) Deacon et al, (2011), (14) Artigau et al. (2010), (15) Lodieu et al. (2007), (16) Tinney et al. (2005), (17) Burgasser et al., (2006a), (18) Burgasser et al. (1999), (19) Wright et al. (2012), (20)

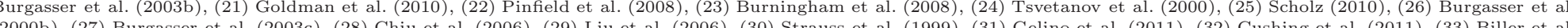

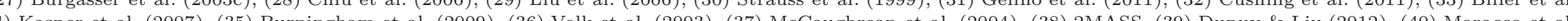
2010) (41) van Leeuwen (2007), (42) Vrba et al (2004), (43) Marsh et al. (in prep.), (44) Schilbach et al (2009), (45) Burgasser et al (2008), (46) Perryman et al (1997), (47) Tinney et al (2003), (48) Henry et al (2006), (49) van Altena et al 1995), (50) Beichman et al. (in prep.), (51) this paper, (52) Burgasser et al 2006al), (53) Burgasser et al (2010), (54) Murray et al. (2011), (55) Delorme et al. (2010), (56) Liu et al.' (2011a), (57) Karatas et al. (2004).

${ }^{\mathrm{a}} \mathrm{An} H$-band magnitude error of $0.1 \mathrm{mag}$ is assigned here since its value is not specified in McCaughrean et al. (2004).

b The association of SDSS J175805.46+463311.9 to G 204-39 - the latter of which has a measured parallax - was made by Faherty et al. (2010).

${ }^{\mathrm{c}}$ See discussion in Dupuy \& Liu (2012) regarding the discrepancy between the parallax value quoted here and that determined by (Tinney et al. (2003).

dCushing et al. 2011) assigns this binary a composite type of T9 on the revised late-T dwarf classification scheme. Given the Dupuy \& Liu (2012) measurements of the parallax and the Liu et al. (2011a) $H$-band measurements of the individual components, we infer spectral types of T8.5 and T9.5 based on absolute $H$ magnitudes of 17.8 and 20.2 mag. 
Table 7. Photometry for Objects in the All-sky Late-T and Y Dwarf Census

\begin{tabular}{|c|c|c|c|c|c|c|c|c|c|}
\hline $\begin{array}{c}\text { Discovery name } \\
\text { (1) }\end{array}$ & $\begin{array}{l}\text { Disc. } \\
\text { ref. } \\
(2)\end{array}$ & $\begin{array}{c}\text { WISE designation }^{\mathrm{a}} \\
\text { (3) }\end{array}$ & $\begin{array}{l}\text { Spec. } \\
\text { type } \\
(4)\end{array}$ & $\begin{array}{l}\text { Type } \\
\text { ref. } \\
(5)\end{array}$ & $\underset{(6)}{\mathrm{H}}$ & $\begin{array}{l}\mathrm{H} \\
\text { ref. } \\
(7)\end{array}$ & $\begin{array}{c}\mathrm{W} 2 \\
(\mathrm{mag}) \\
(8)\end{array}$ & $\begin{array}{c}\text { W1-W2 } \\
(\mathrm{mag}) \\
(9)\end{array}$ & $\begin{array}{c}\mathrm{H}-\mathrm{W} 2 \\
(\mathrm{mag}) \\
(10)\end{array}$ \\
\hline
\end{tabular}

T6-T6.5 Dwarfs

WISEPC J000849.76-173922.6 2MASS J00345157+0523050 WISE J003830.54+840517.

WISE J013525.64+171503.4

CFPA J022105.94+384202.9 CFBDS J022638-072831 2MASSI J0243137-245329 CFBDS J025401-182529 CFBDS J030130+490142.5 CFBDS J03020 - 144504

WISEPA J030533.54+395434.4 ISEPA J030724.57+290447.6 CFHT J0344+3206

WISEA J01054. WISEPA J052536.28+673952. SOri70

WISEPA J054231.26-162829.1 WISEPA J061213.93-303612.7 WISEPA J061407.49+391236.4 WISEPA J062542.21+564625.5 WISEPA J062720.07-111428.8 WISE J073347.94+754439.2 DENIS J081730.0-615520 ULAS J081948.09+073323.3 ULAS J085342.94+000651.8 ULAS J085715.96+091325.3 ISEPC J092906.77+040957.9 2MASSI J0937347+293142 WISEPA J101905.63+195295.3 ULAS J10305. $52-002954.2$ CFBDS J104209+58056 2MASSI J1047538+212423

WISEPC J112254.73+255021.5 ULAS J115038.79+094942.9 ULAS J115338.74-014724.1 NTTDF1205-0744

ULAS J120744.65+133902.7 2MASS J12095613-1004008 SDSS J121440.95+631643.4B WISE J122558.86-101345.0 2MASS J12373919+6526148 WISEPC J132004.16+603426.2
WISE J000849.75-173922.9 WISE J003452.02+052307.0 WISE J003830.54+840517. WISE J013525.64+171503. WISE J022105.97+384203.2 not detected

WISE J024313.47-245331.9 WISE J025401.70-182528. 8 WISE J030013.76+490141. J030130.46-104504
not detected

WISE J030533.51+395434. WISE J030724.59+290447.4 not detected

1131.

WISE J051317. $27+060814.7$ WISE J052536.35+673952. not detected

WISE J054231.27-162829.1 WISE J061213.85-303612.5 WISE J061407.49+391235.9 WISE J062542.22+564625.4 WISE J062720.07-111428. WISE J073347.94+754439.2 WISE J081729.74-615504.0 WISE J085342.83+000650. not detected

WISE J092906.77+040957.9 WISE J093735.61+293127.7 WISE J095259.29+195508. ISE J101905.62+652954.2 not detected

580856.0

WISE J104752.37+212417.5 WISE J112254.72+255022. not detected not detected

WISE J120744.63+133903.8 not detected

blended w/ component A WISE J122558.86-101345.0 WISE J123737.38+652608. WISE J132004.16+603426.3

T6
T6.5
T6
T6
T6.5
T6.5
T6
T6.5
T6.5
T6
T6
T6
T6.5
(T6:)
T6
T6.5
T6p
T6:
T6.5
T6
T6
T6
T6
T6
T6
T6p
T6p
T6
T6.5
T6p
T6
T6
T6.5p
T6.5
T6.5
T6
T6.5p
T6
T6:
T6
T6.5:
(T6)
T6
T6.5
T6.5

$\begin{array}{ccccc}17.77 \pm 0.13 & 1 & 14.56 \pm 0.07 & 1.86 \pm 0.12 & 3.22 \pm 0.15 \\ 15.44 \pm 0.08 & 57 & 12.52 \pm 0.03 & 2.54 \pm 0.05 & 2.92 \pm 0.09 \\ - & - & 15.34 \pm 0.09 & 2.19 \pm 0.20 & - \\ - & - & 14.74 \pm 0.07 & 2.45 \pm 0.17 & - \\ 17.45 \pm 0.15 & 1 & 14.70 \pm 0.08 & 2.18 \pm 0.17 & 2.76 \pm 0.17 \\ 18.65 \pm 0.02 & 56 & - & - & - \\ 15.14 \pm 0.11 & 57 & 12.92 \pm 0.03 & 1.75 \pm 0.04 & 2.21 \pm 0.11 \\ 18.49 \pm 0.05 & 56 & 15.72 \pm 0.12 & >2.64 & 2.77 \pm 0.13 \\ 18.37 \pm 0.16 & 58 & 15.91 \pm 0.20 & >2.28 & 2.46 \pm 0.26 \\ - & - & 15.32 \pm 0.11 & 1.72 \pm 0.16 & - \\ 17.97 \pm 0.04 & 56 & - & - & - \\ 17.08 \pm 0.12 & 1 & 14.69 \pm 0.07 & 2.69 \pm 0.25 & 2.39 \pm 0.14 \\ 17.70 \pm 0.14 & 1 & 14.99 \pm 0.11 & 2.23 \pm 0.24 & 2.71 \pm 0.18 \\ 21.65 \pm 0.08 & 55 & - & - & - \\ 17.26 \pm 0.12 & 1 & 15.08 \pm 0.10 & 1.95 \pm 0.19 & 2.19 \pm 0.16 \\ 16.13 \pm 0.08 & 1 & 13.86 \pm 0.05 & 1.97 \pm 0.08 & 2.28 \pm 0.09 \\ 17.87 \pm 0.05 & 1 & 15.04 \pm 0.09 & >2.94 & 2.83 \pm 0.11 \\ 20.42 \pm 0.11 & 8 & - & - & - \\ 16.57 \pm 0.10 & 1 & 13.98 \pm 0.05 & 2.41 \pm 0.10 & 2.59 \pm 0.11 \\ 17.06 \pm 0.11 & 1 & 14.03 \pm 0.04 & 2.32 \pm 0.08 & 3.03 \pm 0.12 \\ 16.36 \pm 0.25 & 1 & 13.65 \pm 0.04 & 2.83 \pm 0.12 & 2.71 \pm 0.25 \\ 16.90 \pm 0.10 & 1 & 14.38 \pm 0.06 & 1.95 \pm 0.10 & 2.52 \pm 0.12 \\ 15.44 \pm 0.08 & 1 & 13.26 \pm 0.03 & 1.74 \pm 0.05 & 2.18 \pm 0.08 \\ - & - & 14.77 \pm 0.07 & 2.39 \pm 0.16 & - \\ 13.53 \pm 0.03 & 57 & 11.24 \pm 0.02 & 1.72 \pm 0.03 & 2.29 \pm 0.04 \\ 18.36 \pm 0.03 & 10 & - & - & - \\ 19.21 \pm 0.06 & 58 & 15.79 \pm 0.19 & 2.23 \pm 0.42 & 3.42 \pm 0.20 \\ 18.89 \pm 0.10 & 10 & - & - & - \\ 17.37 \pm 0.07 & 1 & 14.23 \pm 0.06 & 2.40 \pm 0.13 & 3.14 \pm 0.09 \\ 14.70 \pm 0.07 & 57 & 11.66 \pm 0.02 & 2.42 \pm 0.04 & 3.05 \pm 0.07 \\ 17.22 \pm 0.10 & 1 & 14.34 \pm 0.06 & 2.53 \pm 0.17 & 2.88 \pm 0.12 \\ 16.52 \pm 0.12 & 1 & 14.03 \pm 0.04 & 2.39 \pm 0.08 & 2.49 \pm 0.12 \\ 19.00 \pm 0.03 & 10 & - & - & - \\ 18.21 \pm 0.05 & 56 & 15.30 \pm 0.10 & 1.95 \pm 0.18 & 2.91 \pm 0.11 \\ 15.80 \pm 0.12 & 57 & 12.97 \pm 0.03 & 2.46 \pm 0.05 & 2.83 \pm 0.12 \\ 16.64 \pm 0.11 & 1 & 13.97 \pm 0.05 & 2.22 \pm 0.10 & 2.67 \pm 0.12 \\ 19.23 \pm 0.06 & 12 & - & - & - \\ 17.97 \pm 0.02 & 10 & - & - & - \\ - & & - & - & - \\ 18.52 \pm 0.05 & 58 & 15.79 \pm 0.18 & >2.08 & 2.73 \pm 0.18 \\ 18.08 \pm 0.26 & 52 & - & - & - \\ - & - & - & - & - \\ 16.46 \pm 0.15 & 2 & 13.99 \pm 0.05 & 2.35 \pm 0.10 & - \\ 15.74 \pm 0.15 & 57 & 12.95 \pm 0.03 & 2.54 \pm 0.05 & 2.79 \pm 0.15 \\ 16.56 \pm 0.13 & 1 & 14.41 \pm 0.05 & 2.52 \pm 0.12 & 2.15 \pm 0.14 \\ & & & & \\ & & & \end{array}$


Table 7-Continued

\begin{tabular}{|c|c|c|c|c|c|c|c|c|c|}
\hline $\begin{array}{l}\text { Discovery name } \\
\text { (1) }\end{array}$ & $\begin{array}{l}\text { Disc. } \\
\text { ref. } \\
(2)\end{array}$ & $\begin{array}{c}\text { WISE designation }{ }^{\mathrm{a}} \\
\text { (3) }\end{array}$ & $\begin{array}{l}\text { Spec. } \\
\text { type } \\
(4)\end{array}$ & $\begin{array}{l}\text { Type } \\
\text { ref. } \\
(5)\end{array}$ & $\begin{array}{c}\mathrm{H} \\
(\mathrm{mag}) \\
(6)\end{array}$ & $\begin{array}{l}\mathrm{H} \\
\text { ref. } \\
(7)\end{array}$ & $\begin{array}{c}\text { W2 } \\
(\mathrm{mag}) \\
(8)\end{array}$ & $\begin{array}{l}\text { W1-W2 } \\
(\text { mag) } \\
(9)\end{array}$ & $\begin{array}{c}\mathrm{H}-\mathrm{W} 2 \\
(\mathrm{mag}) \\
(10)\end{array}$ \\
\hline ULAS J132605.18+120009.9 & 10 & WISE J132605.25+120010.2 & T6p & 10 & $17.93 \pm 0.09$ & 58 & $15.38 \pm 0.10$ & $1.59 \pm 0.16$ & $2.55 \pm 0.14$ \\
\hline SDSSp J134646.45-003150.4 & 14 & WISE J134646.07-003151.4 & $\mathrm{T} 6.5$ & 5 & $15.46 \pm 0.12$ & 57 & $13.57 \pm 0.03$ & $1.92 \pm 0.06$ & $1.89 \pm 0.12$ \\
\hline ULAS J144555.24+125735.1 & 10 & not detected & $\mathrm{T} 6.5$ & 10 & $19.10 \pm 0.05$ & 10 & - & & \\
\hline ULAS J150457.66+053800. $8^{\mathrm{c}}$ & 51 & WISE J150457.57+053759.8 & T6:p & 51 & $17.05 \pm 0.03$ & 51 & $14.23 \pm 0.04$ & $2.25 \pm 0.09$ & $2.32 \pm 0.05$ \\
\hline WISEPC J150649.97+702736.0 & 1 & WISE J150649.98+702736.1 & $\mathrm{T} 6$ & 1 & $13.91 \pm 0.04$ & 1 & $11.27 \pm 0.02$ & $2.12 \pm 0.03$ & $2.64 \pm 0.04$ \\
\hline ULAS J152526.25+095814.3 & 10 & WISE J152526.19+095814.9 & T6.5 & 10 & $19.17 \pm 0.03$ & 58 & $16.44 \pm 0.22$ & $>2.50$ & $2.73 \pm 0.22$ \\
\hline ULAS J152912.23+092228.5 & 10 & not detected & $\mathrm{T} 6$ & 10 & $19.13 \pm 0.05$ & 10 & & & - \\
\hline 2MASSI J1553022+153236A & 6 & WISE J155301.95+153238.8 & $\mathrm{T} 6.5$ & 65 & $16.34 \pm 0.03$ & 65 & $13.02 \pm 0.03^{\mathrm{g}}$ & $2.28 \pm 0.06^{\mathrm{g}}$ & \\
\hline WISEPA J161215.94-342027.1 & 1 & WISE J161215.93-342028.5 & $\mathrm{T} 6.5$ & 1 & $16.96 \pm 0.03$ & 45 & $13.96 \pm 0.05$ & $>4.23$ & $3.00 \pm 0.06$ \\
\hline 2MASS J16150413+1340079 & 15 & WISE J161504.36+134004.2 & $\mathrm{T} 6$ & 15 & $16.49 \pm 0.25$ & 57 & $14.19 \pm 0.05$ & $2.08 \pm 0.10$ & $2.30 \pm 0.26$ \\
\hline WISEPA J162208.94-095934.6 & 1 & WISE J162208.94-095934.4 & $\mathrm{T} 6$ & 1 & $16.05 \pm 0.05$ & 1 & $14.17 \pm 0.06$ & $2.34 \pm 0.15$ & $1.88 \pm 0.08$ \\
\hline SDSSp J162414.37+002915.6 & 16 & WISE J162414.09+002915.6 & $\mathrm{T} 6$ & 5 & $15.52 \pm 0.10$ & 57 & $13.09 \pm 0.03$ & $2.04 \pm 0.05$ & $2.44 \pm 0.11$ \\
\hline WISEPA J162725.64+325525.5 & 1,3 & WISE J162725.65+325524.6 & $\mathrm{T} 6$ & 1 & $16.40 \pm 0.05$ & 1 & $13.60 \pm 0.04$ & $2.65 \pm 0.08$ & $2.80 \pm 0.06$ \\
\hline WISE J172134.46+111739.4 & 2 & WISE J172134.46+111739.4 & $\mathrm{T} 6$ & 2 & - & - & $14.28 \pm 0.06$ & $1.62 \pm 0.09$ & - \\
\hline WISEPA J172844.93+571643.6 & 1 & WISE J172844.93+571642.7 & $\mathrm{T} 6$ & 1 & $17.88 \pm 0.07$ & 1 & $15.01 \pm 0.05$ & $2.94 \pm 0.19$ & $2.87 \pm 0.09$ \\
\hline WISE J174640.78-033818.0 & 2 & WISE J174640.78-033818.0 & T6 & 2 & $17.45 \pm 0.04$ & 2 & $14.79 \pm 0.10$ & $>3.23$ & $2.66 \pm 0.11$ \\
\hline SDSS J175805.46+463311.9 & 17 & WISE J175805.47+463316.8 & $\mathrm{T} 6.5$ & 5 & $16.25 \pm 0.22$ & 57 & $13.82 \pm 0.03$ & $1.86 \pm 0.05$ & $2.43 \pm 0.22$ \\
\hline SCR J1845-6357B & 18 & blended w/ SCR J1845-6357A & $\mathrm{T} 6$ & 19 & - & - & & - & - \\
\hline WISEPA J190624.75+450808.2 & 1 & WISE J190624.74+450807.1 & $\mathrm{T} 6$ & 1 & $16.32 \pm 0.09$ & 1 & $13.82 \pm 0.04$ & $2.27 \pm 0.07$ & $2.51 \pm 0.10$ \\
\hline WISE J192841.35+235604.9 & 2 & WISE J192841.35+235604.9 & $\mathrm{T} 6$ & 2 & - & - & $12.11 \pm 0.03$ & $1.83 \pm 0.04$ & - \\
\hline$\epsilon$ Ind $\mathrm{Bb}$ & 20 & blended $\mathrm{w} / \epsilon$ Ind $\mathrm{Ba}$ & $\mathrm{T} 6$ & 5 & - & - & - & - & - \\
\hline 2MASS J21543318+5942187 & 15 & WISE J215432.95+594213.8 & $\mathrm{T} 6$ & 15 & $15.77 \pm 0.17$ & 57 & $13.51 \pm 0.03$ & $2.14 \pm 0.08$ & $2.26 \pm 0.17$ \\
\hline UDXS J221903.10+002418.2 & 54 & not detected & (T6:) & 54 & - & - & - & - & - \\
\hline 2MASS J22282889-4310262 & 21 & WISE J222829.00-431029.5 & $\mathrm{T} 6$ & 5 & $15.36 \pm 0.12$ & 57 & $13.33 \pm 0.04$ & $1.94 \pm 0.06$ & $2.04 \pm 0.12$ \\
\hline WISE J223720.39+722833.8 & 2 & WISE J223720.39+722833.8 & T6 & 2 & - & - & $13.61 \pm 0.04$ & $2.06 \pm 0.07$ & - \\
\hline WISE J230133.32+021635.1 & 2 & WISE J230133.32+021635.1 & $\mathrm{T} 6.5$ & 2 & - & - & $14.34 \pm 0.06$ & $1.96 \pm 0.10$ & - \\
\hline ULAS J230601.02+130225.0 & 10 & WISE J230601.02+130225.3 & T6.5 & 10 & $18.00 \pm 0.02$ & 58 & $15.07 \pm 0.10$ & $3.33 \pm 0.51$ & $2.94 \pm 0.10$ \\
\hline ULAS J231557.61+132256.2 & 10 & WISE J231557.66+132255.5 & $\mathrm{T} 6.5$ & 10 & $18.16 \pm 0.05$ & 58 & $15.47 \pm 0.13$ & $2.36 \pm 0.34$ & $2.69 \pm 0.14$ \\
\hline CFBDS J232304-015232 & 56 & WISE J232304.41-015233.8 & $\mathrm{T} 6$ & 56 & $17.46 \pm 0.04$ & 56 & $15.07 \pm 0.11$ & $1.61 \pm 0.17$ & $2.39 \pm 0.12$ \\
\hline WISE J234228.98+085620.2 & 2 & WISE J234228.98+085620.2 & T6.5 & 2 & - & - & $13.97 \pm 0.05$ & $2.10 \pm 0.09$ & - \\
\hline WISEPA J234351.20-741847.0 & 1 & WISE J234351.20-741846.9 & $\mathrm{T} 6$ & 1 & $>16.14$ & 1 & $13.73 \pm 0.04$ & $2.01 \pm 0.06$ & $>2.41$ \\
\hline WISE J235716.49+122741.8 & 2 & WISE J235716.49+122741.8 & T6 & 2 & 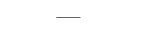 & - & $13.99 \pm 0.05$ & $1.85 \pm 0.08$ & 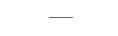 \\
\hline \multicolumn{10}{|c|}{ T7-T7.5 Dwarfs } \\
\hline HD $3651 \mathrm{~B}$ & 22,23 & blended w/ HD 3651A & $\mathrm{T} 7.5$ & 23 & $16.75 \pm 0.16$ & 22 & - & - & - \\
\hline WISE J004024.88+090054.8 & 2 & WISE J004024.88+090054.8 & $\mathrm{T} 7$ & 2 & - & - & $13.92 \pm 0.07$ & $2.14 \pm 0.11$ & - \\
\hline 2MASS J00501994-3322402 & 24 & WISE J005021.03-332228.9 & $\mathrm{T} 7$ & 5 & $15.84 \pm 0.19$ & 57 & $13.55 \pm 0.04$ & $1.99 \pm 0.06$ & $2.29 \pm 0.19$ \\
\hline WISEPA J012333.21+414203.9 & 1 & WISE J012333.23+414203.8 & $\mathrm{T} 7$ & 1 & $17.20 \pm 0.13$ & 1 & $15.03 \pm 0.07$ & $2.01 \pm 0.14$ & $2.18 \pm 0.15$ \\
\hline ULAS J013939.77+004813.8 & 25 & not detected & $\mathrm{T} 7.5$ & 25 & $19.12 \pm 0.05$ & 25 & - & - & - \\
\hline ULAS J015024.37+135924.0 & 10 & WISE J015024.39+135923.8 & $\mathrm{T} 7.5$ & 10 & $18.11 \pm 0.02$ & 58 & $15.24 \pm 0.09$ & $2.75 \pm 0.30$ & $2.87 \pm 0.10$ \\
\hline WISEPC J022322.39-293258.1 & 1 & WISE J022322.36-293257.1 & $\mathrm{T} 7.5$ & 1 & $17.30 \pm 0.11$ & 1 & $14.03 \pm 0.04$ & $3.11 \pm 0.13$ & $3.27 \pm 0.12$ \\
\hline WISEPA J022623.98-021142.8 & 1 & WISE J022623.98-021142.8 & $\mathrm{T} 7$ & 1 & $18.84 \pm 0.24$ & 45 & $14.59 \pm 0.06$ & $3.01 \pm 0.20$ & - \\
\hline WISE J024124.73-365328.0 & 2 & WISE J024124.73-365328.0 & $\mathrm{T} 7$ & 2 & $16.98 \pm 0.09$ & 45 & $14.34 \pm 0.04$ & $2.55 \pm 0.11$ & - \\
\hline CFBDS J030135-161418 & 56 & WISE J030135.16-161417.8 & $\mathrm{T} 7$ & 56 & $18.99 \pm 0.10$ & 56 & $15.59 \pm 0.10$ & $3.07 \pm 0.41$ & $3.40 \pm 0.14$ \\
\hline WISE J032547.72+083118.2 & 2 & WISE J032547.72+083118.2 & $\mathrm{T} 7$ & 2 & $16.15 \pm 0.08$ & 45 & $13.49 \pm 0.04$ & $1.95 \pm 0.07$ & $2.66 \pm 0.09$ \\
\hline 2MASS J03480772-602227 & 21 & WISE J034807.33-602234.9 & $\mathrm{T} 7$ & 5 & $15.56 \pm 0.14$ & 57 & $12.54 \pm 0.02$ & $2.47 \pm 0.04$ & $3.02 \pm 0.15$ \\
\hline WISEPA J052844.51-330823.9 & 1 & WISE J052844.51-330823.9 & $\mathrm{T} 7 \mathrm{p}$ & 1 & $16.97 \pm 0.14$ & 1 & $14.60 \pm 0.06$ & $3.13 \pm 0.24$ & $2.37 \pm 0.15$ \\
\hline
\end{tabular}


Table 7-Continued

\begin{tabular}{|c|c|c|c|c|c|c|c|c|c|}
\hline $\begin{array}{c}\text { Discovery name } \\
\text { (1) }\end{array}$ & $\begin{array}{l}\text { Disc. } \\
\text { ref. } \\
(2)\end{array}$ & $\begin{array}{l}\text { WISE designation }{ }^{\mathrm{a}} \\
\text { (3) }\end{array}$ & $\begin{array}{l}\text { Spec. } \\
\text { type } \\
(4)\end{array}$ & $\begin{array}{l}\text { Type } \\
\text { ref. } \\
(5)\end{array}$ & $\begin{array}{c}\mathrm{H} \\
(\mathrm{mag}) \\
(6)\end{array}$ & $\begin{array}{l}\mathrm{H} \\
\text { ref. } \\
(7)\end{array}$ & $\begin{array}{c}\mathrm{W} 2 \\
(\mathrm{mag}) \\
(8)\end{array}$ & $\begin{array}{l}\text { W1-W2 } \\
(\operatorname{mag}) \\
(9)\end{array}$ & $\begin{array}{c}\mathrm{H}-\mathrm{W} 2 \\
(\mathrm{mag}) \\
(10)\end{array}$ \\
\hline UGCS J053022.52-052447.4 & 7 & not detected & T7: & 7 & $19.13 \pm 0.29$ & 7 & - & - & - \\
\hline G1229B & 27 & blended w/ G1 229A & $\mathrm{T} 7 \mathrm{p}$ & 5 & $\sim 14.3$ & 63 & - & - & - \\
\hline WISE J061437.73+095135.0 & 2 & WISE J061437.73+095135.0 & $\mathrm{T} 7$ & 2 & $16.82 \pm 0.10$ & 45 & $14.29 \pm 0.07$ & $2.63 \pm 0.20$ & $2.53 \pm 0.12$ \\
\hline 2MASSI J0727182+171001 & 6 & WISE J072719.13+170951.6 & $\mathrm{T} 7$ & 5 & $15.76 \pm 0.17$ & 57 & $12.96 \pm 0.03$ & $2.28 \pm 0.06$ & $2.79 \pm 0.17$ \\
\hline ULAS J085910.69+101017.1 & 12 & WISE J085910.63+101014.8 & $\mathrm{T} 7$ & 12 & $18.58 \pm 0.06$ & 58 & $15.28 \pm 0.14$ & $2.44 \pm 0.35$ & $3.30 \pm 0.15$ \\
\hline ULAS J090116.23-030635.0 & 28 & WISE J090116.21-030636.0 & $\mathrm{T} 7.5$ & 28 & $18.46 \pm 0.13$ & 58 & $14.60 \pm 0.07$ & $3.17 \pm 0.32$ & $3.86 \pm 0.15$ \\
\hline CFBDS J092250+152741 & 46 & not detected & $\mathrm{T} 7$ & 46 & $18.81 \pm 0.10$ & 56 & - & - & - \\
\hline ULAS J094806.06+064805.0 & 28 & not detected & $\mathrm{T} 7$ & 28 & $19.46 \pm 0.22$ & 28 & - & - & - \\
\hline WISE J103907.73-160002.9 & 2 & WISE J103907.73-160002.9 & $\mathrm{T} 7.5$ & 2 & $17.19 \pm 0.20$ & 45 & $14.16 \pm 0.05$ & $2.49 \pm 0.12$ & $3.03 \pm 0.21$ \\
\hline 2MASS J11145133-2618235 & 24 & WISE J111448.79-261827.7 & $\mathrm{T} 7.5$ & 5 & $15.73 \pm 0.12$ & 57 & $12.24 \pm 0.03$ & $3.13 \pm 0.06$ & $3.50 \pm 0.13$ \\
\hline WISE J112438.12-042149.7 & 2 & WISE J112438.12-042149.7 & $\mathrm{T} 7$ & 2 & - & - & $14.05 \pm 0.05$ & $2.51 \pm 0.13$ & - \\
\hline 2MASSI J1217110-031113 & 11 & WISE J121710.30-031112.1 & $\mathrm{T} 7.5$ & 5 & $15.75 \pm 0.12$ & 57 & $13.20 \pm 0.04$ & $2.09 \pm 0.06$ & $2.55 \pm 0.12$ \\
\hline WISP J1232-0033 & 68 & not detected & $\mathrm{T} 7$ & 68 & - & - & - & - & - \\
\hline ULAS J124804.56+075904.0 & 10 & WISE J124804.49+075903.5 & $\mathrm{T} 7$ & 10 & $18.15 \pm 0.08$ & 58 & $15.37 \pm 0.13$ & $1.91 \pm 0.24$ & $2.78 \pm 0.15$ \\
\hline Kelu-1 $A b^{\mathrm{h}}$ & 60 & blended w/ components $\mathrm{Aa}, \mathrm{B}$ & $(\mathrm{T} 7.5)$ & 60 & - & - & - & - & - \\
\hline ULAS J131508.42+082627.4 & 12 & not detected & T7.5 & 12 & $19.50 \pm 0.10$ & 12 & - & - & - \\
\hline ULAS J134940.81+091833.3 & 10 & not detected & $\mathrm{T} 7$ & 10 & $19.43 \pm 0.03$ & 10 & - & - & - \\
\hline ULAS J141623.94+134836.3 & 29 & WISE J141623.94+134836.0 & $\mathrm{T} 7.5$ & 30 & $17.58 \pm 0.03$ & 58 & $12.79 \pm 0.04$ & $3.33 \pm 0.20$ & $4.79 \pm 0.05$ \\
\hline WISEPC J145715.03+581510.2 & 1 & WISE J145715.03+581510.2 & $\mathrm{T} 7$ & 1 & $16.64 \pm 0.29$ & 1 & $14.47 \pm 0.04$ & $2.36 \pm 0.09$ & $2.17 \pm 0.29$ \\
\hline G1570D & 31 & WISE J145715.84-212207.9 & $\mathrm{T} 7.5$ & 5 & $15.27 \pm 0.09$ & 57 & $12.11 \pm 0.02$ & $2.71 \pm 0.04$ & $3.15 \pm 0.09$ \\
\hline SDSS J150411.63+102718.4 & 32 & WISE J150411.80+102715.7 & $\mathrm{T} 7$ & 32 & - & - & $14.06 \pm 0.04$ & $2.33 \pm 0.08$ & - \\
\hline WISE J152305.10+312537.6 & 2 & WISE J152305.10+312537.6 & T7 p & 2 & $18.69 \pm 0.18$ & 45 & $14.39 \pm 0.04$ & $3.52 \pm 0.22$ & $4.30 \pm 0.18$ \\
\hline WISE J154459.27+584204.5 & 2 & WISE J154459.27+584204.5 & $\mathrm{T} 7.5$ & 2 & $18.34 \pm 0.29$ & 45 & $15.03 \pm 0.06$ & $3.36 \pm 0.31$ & $3.31 \pm 0.30$ \\
\hline 2MASSI J1553022+153236B & $5 ?$ & blended w/ component A & $\mathrm{T} 7.5$ & 65 & $16.72 \pm 0.03$ & 65 & - & - & - \\
\hline SDSS J162838.77+230821.1 & 32 & WISE J162839.00+230818.0 & $\mathrm{T} 7$ & 32 & $16.11 \pm 0.15$ & 57 & $13.96 \pm 0.04$ & $2.47 \pm 0.10$ & $2.15 \pm 0.16$ \\
\hline WISEPA J185215.78+353716.3 & 1 & WISE J185215.76+353716.7 & $\mathrm{T} 7$ & 1 & $>17.41$ & 1 & $14.24 \pm 0.05$ & $2.31 \pm 0.11$ & $>3.17$ \\
\hline WISE J201404.13+042408.5 & 2 & WISE J201404.13+042408.5 & T7 p & 2 & $18.66 \pm 0.30$ & 45 & $15.16 \pm 0.12$ & $>2.78$ & $3.50 \pm 0.32$ \\
\hline WISEPA J201824.96-742325.9 & 26 & WISE J201824.97-742327.5 & $\mathrm{T} 7$ & 26 & $17.17 \pm 0.04$ & - & $13.62 \pm 0.04$ & $2.92 \pm 0.11$ & $3.55 \pm 0.06$ \\
\hline WISEPC J215751.38+265931.4 & 1 & WISE J215751.35+265931.4 & $\mathrm{T} 7$ & 1 & $17.45 \pm 0.04$ & 1 & $14.48 \pm 0.06$ & $2.65 \pm 0.16$ & $2.97 \pm 0.07$ \\
\hline WISEPC J220922.10-273439.5 & 1 & WISE J220922.11-273439.6 & $\mathrm{T} 7$ & 1 & $>16.41$ & 1 & $13.84 \pm 0.05$ & $2.66 \pm 0.12$ & $>2.57$ \\
\hline WISEPC J221354.69+091139.4 & 1 & WISE J221354.68+091139.4 & $\mathrm{T} 7$ & 1 & $17.09 \pm 0.11$ & 1 & $14.60 \pm 0.07$ & $2.10 \pm 0.13$ & $2.49 \pm 0.13$ \\
\hline $\begin{array}{l}\text { UDXS J221611.51+003308.1 } \\
\text { Unt }\end{array}$ & 54 & not detected & (T7.5:) & 54 & $17.00+11$ & - & $1.00 \pm 0.01$ & $2.10+0.10$ & $2.40 \pm$ \\
\hline WISEPC J231939.13-184404.3 & 1 & WISE J231939.14-184404.4 & $\mathrm{T} 7.5$ & 1 & $17.95 \pm 0.05$ & 1 & $13.76 \pm 0.05$ & $3.39 \pm 0.17$ & $4.19 \pm 0.07$ \\
\hline ULAS J232123.79+135454.9 & 33 & WISE J232123.83+135453.5 & $\mathrm{T} 7.5$ & 10 & $17.15 \pm 0.03$ & 58 & $14.10 \pm 0.06$ & $3.21 \pm 0.22$ & $3.05 \pm 0.06$ \\
\hline ULAS J232802.03+134544.8 & 10 & WISE J232802.12+134545.3 & $\mathrm{T} 7$ & 10 & $18.17 \pm 0.02$ & 58 & $15.39 \pm 0.13$ & $2.61 \pm 0.40$ & $2.78 \pm 0.13$ \\
\hline WISE J233543.79+422255.2 & 2 & WISE J233543.79+422255.2 & T7 & 2 & $10.17 \pm .02$ & - & $15.31 \pm 0.10$ & $3.24 \pm 0.52$ & $2.10 \pm 0$ \\
\hline WISEPC J234026.62-074507.2 & 1 & WISE J234026.62-074508.1 & $\mathrm{T} 7$ & 1 & $16.19 \pm 0.06$ & 1 & $13.58 \pm 0.04$ & $2.37 \pm 0.09$ & $2.61 \pm 0.07$ \\
\hline WISEPC J234841.10-102844.4 & 1 & WISE J234841.10-102844.1 & $\mathrm{T} 7$ & 1 & $16.93 \pm 0.12$ & 1 & $14.40 \pm 0.06$ & $2.49 \pm 0.16$ & $2.53 \pm 0.14$ \\
\hline \multicolumn{10}{|c|}{ T8-T8.5 Dwarfs } \\
\hline WISE J001505.87-461517.6 & 2 & WISE J001505.87-461517.6 & $\mathrm{T} 8$ & 2 & - & - & $14.25 \pm 0.06$ & $2.77 \pm 0.16$ & - \\
\hline WISE J003231.09-494651.4 & 2 & WISE J003231.09-494651.4 & $\mathrm{T} 8.5$ & 2 & - & - & $15.07 \pm 0.09$ & $3.01 \pm 0.35$ & - \\
\hline ULAS J003402.77-005206.7 & 35 & WISE J003402.78-005208.1 & $\mathrm{T} 8.5$ & 36 & $18.49 \pm 0.04$ & 58 & $14.50 \pm 0.08$ & $2.97 \pm 0.30$ & $3.99 \pm 0.09$ \\
\hline WISE J004945.61+215120.0 & 2 & WISE J004945.61+215120.0 & $\mathrm{T} 8.5$ & 2 & - & - & $12.97 \pm 0.03$ & $2.50 \pm 0.05$ & - \\
\hline CFBDS J005910.90-011401.3 & 37 & WISE J005911.08-011400.9 & $\mathrm{T} 8.5$ & 36 & $18.27 \pm 0.05$ & 59 & $13.68 \pm 0.04$ & $3.39 \pm 0.16$ & $4.59 \pm 0.07$ \\
\hline CFBDS J013302+023128 & 56 & WISE J013302.44+023128.9 & $\mathrm{T} 8.5$ & 56 & $18.62 \pm 0.10$ & 56 & $15.10 \pm 0.09$ & $2.69 \pm 0.28$ & $3.52 \pm 0.14$ \\
\hline WISE J024512.62-345047.8 & 2 & WISE J024512.62-345047.8 & T8 & 2 & - & - & $14.56 \pm 0.06$ & $3.36 \pm 0.32$ & \\
\hline
\end{tabular}


Table 7-Continued

\begin{tabular}{|c|c|c|c|c|c|c|c|c|c|}
\hline $\begin{array}{l}\text { Discovery name } \\
\text { (1) }\end{array}$ & $\begin{array}{l}\text { Disc. } \\
\text { ref. } \\
(2)\end{array}$ & $\begin{array}{c}\text { WISE designation }{ }^{\mathrm{a}} \\
\text { (3) }\end{array}$ & $\begin{array}{l}\text { Spec. } \\
\text { type } \\
(4)\end{array}$ & $\begin{array}{l}\text { Type } \\
\text { ref. } \\
(5)\end{array}$ & $\begin{array}{c}\mathrm{H} \\
(\mathrm{mag}) \\
(6)\end{array}$ & $\begin{array}{l}\mathrm{H} \\
\text { ref. } \\
(7)\end{array}$ & $\begin{array}{l}\text { W2 } \\
(\mathrm{mag}) \\
(8)\end{array}$ & $\begin{array}{l}\text { W1-W2 } \\
(\mathrm{mag}) \\
(9)\end{array}$ & $\begin{array}{c}\mathrm{H}-\mathrm{W} 2 \\
(\mathrm{mag}) \\
(10)\end{array}$ \\
\hline WISE J024714.52+372523.5 & 2 & WISE J024714.52+372523.5 & $\mathrm{T} 8$ & 2 & $18.24 \pm 0.19$ & 2 & $14.70 \pm 0.08$ & $>3.67$ & $3.54 \pm 0.21$ \\
\hline WISEPA J025409.45+022359.1 & 1,42 & WISE J025409.51+022358.6 & $\mathrm{T} 8$ & 1 & $16.29 \pm 0.04$ & 1 & $12.74 \pm 0.03$ & $3.02 \pm 0.06$ & $3.55 \pm 0.05$ \\
\hline WISEPA J031325.96+780744.2 & 1 & WISE J031325.94+780744.3 & $\mathrm{T} 8.5$ & 1 & $17.63 \pm 0.06$ & 1 & $13.19 \pm 0.03$ & $2.69 \pm 0.07$ & $4.45 \pm 0.07$ \\
\hline WISE J031624.35+430709.1 & 2 & WISE J031624.35+430709.1 & T8 & 2 & $19.70 \pm 0.09$ & 45 & $14.56 \pm 0.07^{\mathrm{j}}$ & $>3.30^{\mathrm{j}}$ & $5.14 \pm 0.11^{\mathrm{j}}$ \\
\hline WISE J032120.91-734758.8 & 2 & WISE J032120.91-734758.8 & $\mathrm{T} 8$ & 2 & $19.06 \pm 0.12$ & 2 & $15.60 \pm 0.08$ & $>3.59$ & - \\
\hline WISEPC J032337.53-602554.9 & 1 & WISE J032337.53-602554.5 & $\mathrm{T} 8.5$ & 1 & $18.40 \pm 0.02$ & 1 & $14.50 \pm 0.05$ & $3.61 \pm 0.33$ & $3.90 \pm 0.06$ \\
\hline WISE J033605.05-014350.4 & 2 & WISE J033605.05-014350.4 & T8:: & 2 & - & - & $14.54 \pm 0.07$ & $>3.423$ & - \\
\hline 2MASSW J0337036-175807B & 53 & blended w/ component A & (T8??) & 53 & - & - & - & - & - \\
\hline 2MASSI J0415195-093506 & 6 & WISE J041521.21-093500.6 & $\mathrm{T} 8$ & 5 & $15.54 \pm 0.11$ & 57 & $12.26 \pm 0.03$ & $2.85 \pm 0.05$ & $3.28 \pm 0.12$ \\
\hline WISEPA J045853.89+643452.9A & 34 & WISE J045853.89+643452.5 & $\mathrm{T} 8.5$ & 61 & $17.41 \pm 0.06^{\mathrm{g}}$ & 1 & $13.03 \pm 0.03^{\mathrm{g}}$ & $3.64 \pm 0.12^{\mathrm{g}}$ & $4.38 \pm 0.07^{\mathrm{g}}$ \\
\hline WISEPA J050003.05-122343.2 & 1 & WISE J050003.04-122343.2 & T8 & 1 & $18.13 \pm 0.12$ & 1 & $13.94 \pm 0.04$ & $3.72 \pm 0.25$ & $4.19 \pm 0.13$ \\
\hline WISEPA J062309.94-045624.6 & 1 & WISE J062309.94-045624.6 & T8 & 1 & $17.31 \pm 0.11$ & 1 & $13.82 \pm 0.04$ & $3.52 \pm 0.20$ & $3.50 \pm 0.12$ \\
\hline 2MASS J07290002-3954043 & 15 & WISE J072859.49-395345.7 & $\mathrm{T} 8 \mathrm{p}$ & 15 & $15.98 \pm 0.18$ & 57 & $12.96 \pm 0.03$ & $2.29 \pm 0.05$ & $3.02 \pm 0.19$ \\
\hline WISEPA J074457.15+562821.8 & 1 & WISE J074457.25+562821.0 & $\mathrm{T} 8$ & 1 & $17.59 \pm 0.12$ & 1 & $14.49 \pm 0.06$ & $2.59 \pm 0.16$ & $3.10 \pm 0.13$ \\
\hline WISEPA J075003.84+272544.8 & 1 & WISE J075003.78+272545.4 & $\mathrm{T} 8.5$ & 1 & $19.00 \pm 0.06$ & 1 & $14.46 \pm 0.07$ & $>3.65$ & $4.54 \pm 0.09$ \\
\hline WISEPC J075946.98-490454.0 & 1 & WISE J075946.98-490454.0 & T8 & 1 & $17.41 \pm 0.04$ & 45 & $13.86 \pm 0.04$ & $>4.05$ & $3.55 \pm 0.05$ \\
\hline WISE J081220.04+402106.2 & 2 & WISE J081220.04+402106.2 & $\mathrm{T} 8$ & 2 & $18.30 \pm 0.20$ & 2 & $15.08 \pm 0.10$ & $3.16 \pm 0.48$ & $3.22 \pm 0.22$ \\
\hline WISEPC J083641.12-185947.2 & 1 & WISE J083641.10-185947.0 & $\mathrm{T} 8 \mathrm{p}$ & 1 & $18.79 \pm 0.26$ & 45 & $15.18 \pm 0.10$ & $>3.23$ & $3.61 \pm 0.28$ \\
\hline WISEPA J085716.25+560407.6 & 1 & WISE J085716.24+560407.6 & T8 & 1 & $17.49 \pm 0.14$ & 1 & $14.11 \pm 0.05$ & $3.37 \pm 0.22$ & $3.38 \pm 0.15$ \\
\hline WISEPA J090649.36+473538.6 & 1 & WISE J090649.35+473538.5 & $\mathrm{T} 8$ & 1 & $17.81 \pm 0.16$ & 1 & $14.65 \pm 0.08$ & $2.83 \pm 0.24$ & $3.17 \pm 0.18$ \\
\hline 2MASS J09393548-2448279 & 24 & WISE J093935.91-244838.5 & $\mathrm{T} 8$ & 5 & $15.78 \pm 0.15$ & 57 & $11.64 \pm 0.02$ & $3.39 \pm 0.05$ & $4.16 \pm 0.15$ \\
\hline ULAS J101721.40+011817.9 & 38 & not detected & $\mathrm{T} 8 \mathrm{p}$ & 38 & $19.07 \pm 0.02$ & 38 & - & - & - \\
\hline WISEPC J101808.05-244557.7 & 1 & WISE J101808.04-244557.9 & T8 & 1 & $18.00 \pm 0.23$ & 45 & $14.17 \pm 0.05$ & $3.21 \pm 0.22$ & $3.83 \pm 0.24$ \\
\hline CFBDS J102841+565401 $\mathrm{e}^{\mathrm{e}}$ & 46 & not detected & $\mathrm{T} 8$ & 46 & $18.38 \pm 0.08$ & 56 & - & - & - \\
\hline WISEPC J104245.23-384238.3 & 1 & WISE J104245.23-384238.3 & $\mathrm{T} 8.5$ & 1 & $19.08 \pm 0.11$ & 45 & $14.52 \pm 0.06$ & $>4.06$ & $4.56 \pm 0.13$ \\
\hline$\xi$ UMa C & 67 & WISE J111838.70+312537.9 & $\mathrm{T} 8.5$ & 67 & $18.15 \pm 0.06$ & 67 & $13.31 \pm 0.03$ & $2.85 \pm 0.08$ & $2.28 \pm 0.06$ \\
\hline WISEPC J115013.88+630240.7 & 1 & WISE J115013.85+630241.5 & T8 & 1 & $>18.01$ & 1 & $13.40 \pm 0.03$ & $3.59 \pm 0.11$ & $>4.61$ \\
\hline 2MASS J12255432-2739466B & 64 & blended w/ component A & $\mathrm{T} 8$ & 64,65 & $16.91 \pm 0.03$ & 65 & & - & - \\
\hline ULAS J123828.51+095351.3 & 38 & WISE J123828.38+095352.1 & $\mathrm{T} 8$ & 36 & $19.20 \pm 0.02$ & 58 & $15.22 \pm 0.12$ & $>2.79$ & $3.98 \pm 0.12$ \\
\hline Ross $458 \mathrm{C}$ & 39 & WISE J130041.65+122114.6 & $\mathrm{T} 8$ & 36 & $17.01 \pm 0.04$ & 58 & $13.74 \pm 0.04$ & $2.27 \pm 0.08$ & $3.27 \pm 0.06$ \\
\hline ULAS J130217.21+130851.2 & 10 & WISE J130217.08+130851.0 & $\mathrm{T} 8$ & 36 & $18.60 \pm 0.06$ & 58 & $14.95 \pm 0.10$ & $>2.66$ & $3.65 \pm 0.11$ \\
\hline WISEPA J132233.66-234017.1 & 1,3 & WISE J132233.64-234016.8 & T8 & 1 & $16.61 \pm 0.14$ & 1 & $13.93 \pm 0.04$ & $3.06 \pm 0.14$ & $2.68 \pm 0.15$ \\
\hline ULAS J133553.45+113005.2 & 38 & WISE J133553.41+113004.8 & $\mathrm{T} 8.5$ & 36 & $18.25 \pm 0.01$ & 58 & $13.87 \pm 0.04$ & $3.01 \pm 0.13$ & $4.39 \pm 0.04$ \\
\hline WISE J143311.42-083736.4 & 2 & WISE J143311.42-083736.4 & T8:: & 2 & $19.42 \pm 0.21$ & 2 & $15.23 \pm 0.10$ & $>3.51$ & $4.19 \pm 0.23$ \\
\hline WISEPA J143602.19-181421.8 & 1 & WISE J143602.20-181421.9 & $\mathrm{T} 8 \mathrm{p}$ & 1 & $>17.62$ & 45 & $14.71 \pm 0.06$ & $2.50 \pm 0.16$ & $>2.91$ \\
\hline CFBDSIR J145829+101343A & 44 & WISE J145829.40+101341.7 & $\mathrm{T} 8.5$ & $45^{\mathrm{i}}$ & $20.18 \pm 0.10$ & 66 & $15.66 \pm 0.12^{\mathrm{g}}$ & $>3.15^{\mathrm{g}}$ & - \\
\hline WISE J151721.13+052929.3 & 2 & WISE J151721.13+052929.3 & $\mathrm{T} 8$ & 2 & $18.85 \pm 0.15$ & 58 & $15.13 \pm 0.08$ & $>2.97$ & $3.72 \pm 0.17$ \\
\hline WISEPC J151906.64+700931.5 & 1 & WISE J151906.63+700931.4 & $\mathrm{T} 8$ & 1 & $18.28 \pm 0.07$ & 1 & $14.14 \pm 0.03$ & $3.03 \pm 0.10$ & $4.15 \pm 0.08$ \\
\hline WISEPA J161705.75+180714.3 & 26 & WISE J161705.74+180714.2 & $\mathrm{T} 8$ & 26 & $18.23 \pm 0.08$ & 1 & $14.16 \pm 0.05$ & $2.96 \pm 0.16$ & $4.08 \pm 0.09$ \\
\hline WISEPA J165311.05+444423.9 & 1,3 & WISE J165311.05+444422.8 & $\mathrm{T} 8$ & 1 & $17.53 \pm 0.05$ & 1 & $13.81 \pm 0.04$ & $2.68 \pm 0.08$ & $3.72 \pm 0.06$ \\
\hline WISEPA J171104.60+350036.8 & 1 & WISE J171104.60+350036.8 & $\mathrm{T} 8$ & 1 & $>18.12$ & 1 & $14.72 \pm 0.06$ & $>3.43$ & $>3.40$ \\
\hline WISEPA J171717.02+612859.3 & 1 & WISE J171717.02+612859.3 & $\mathrm{T} 8$ & 1 & $18.91 \pm 0.09$ & 1 & $15.09 \pm 0.05$ & $3.33 \pm 0.20$ & $3.83 \pm 0.10$ \\
\hline WISE J180901.07+383805.4 & 2 & WISE J180901.07+383805.4 & $\mathrm{T} 8$ & 2 & & - & $15.18 \pm 0.08$ & $>2.84$ & . \\
\hline WISEPA J181210.85+272144.3 & 26 & WISE J181210.85+272144.3 & T8.5: & 1 & $18.83 \pm 0.16$ & 1 & $14.23 \pm 0.05$ & $3.58 \pm 0.29$ & $4.60 \pm 0.17$ \\
\hline WISEPA J195905.66-333833.7 & 1 & WISE J195905.65-333833.5 & $\mathrm{T} 8$ & 1 & $17.18 \pm 0.05$ & 1 & $13.88 \pm 0.05$ & $2.56 \pm 0.11$ & $3.30 \pm 0.07$ \\
\hline WISEPC J201546.27+664645.1 $1^{\mathrm{f}}$ & 2 & blended w/ nearby source & $\mathrm{T} 8$ & 2 & $17.66 \pm 0.23$ & 2 & $14.68 \pm 0.06$ & $2.02 \pm 0.11$ & $1.82 \pm 0.11$ \\
\hline WISE J201920.76-114807.6 & 2 & WISE J201920.76-114807.6 & T8.5: & 2 & $18.32 \pm 0.11$ & 2 & $14.32 \pm 0.06$ & $2.96 \pm 0.22$ & $3.89 \pm 0.11$ \\
\hline Wolf $940 \mathrm{~B}$ & 40 & WISE J214638.99-001039.6 & $\mathrm{T} 8.5$ & 36 & $18.77 \pm 0.03$ & 58 & $14.24 \pm 0.05$ & $2.49 \pm 0.13$ & $4.53 \pm 0.06$ \\
\hline
\end{tabular}


Table 7-Continued

\begin{tabular}{|c|c|c|c|c|c|c|c|c|c|}
\hline $\begin{array}{c}\text { Discovery name } \\
\text { (1) }\end{array}$ & $\begin{array}{l}\text { Disc. } \\
\text { ref. } \\
(2)\end{array}$ & $\begin{array}{c}\text { WISE designation } \\
\text { (3) }\end{array}$ & $\begin{array}{l}\text { Spec. } \\
\text { type } \\
(4)\end{array}$ & $\begin{array}{l}\text { Type } \\
\text { ref. } \\
(5)\end{array}$ & $\begin{array}{c}\mathrm{H} \\
(\mathrm{mag}) \\
(6)\end{array}$ & $\begin{array}{l}\mathrm{H} \\
\text { ref. } \\
(7)\end{array}$ & $\begin{array}{l}\text { W2 } \\
(\mathrm{mag}) \\
(8)\end{array}$ & $\begin{array}{l}\text { W1-W2 } \\
(\mathrm{mag}) \\
(9)\end{array}$ & $\begin{array}{c}\mathrm{H}-\mathrm{W} 2 \\
(\mathrm{mag}) \\
(10)\end{array}$ \\
\hline WISEPC J222623.05+044003.9 & 1 & WISE J222623.05+044004.0 & $\mathrm{T} 8$ & 1 & $16.95 \pm 0.17$ & 45 & $14.71 \pm 0.09$ & $2.58 \pm 0.24$ & $2.24 \pm 0.19$ \\
\hline WISEPC J225540.74-311841.8 & 1 & WISE J225540.75-311842.0 & $\begin{array}{l}10 \\
\mathrm{~T} 8\end{array}$ & 1 & $17.70 \pm 0.11$ & 1 & $14.18 \pm 0.06$ & $2.74 \pm 0.18$ & $3.52 \pm 0.12$ \\
\hline WISEPA J231336.40-803700.3 & 26 & WISE J231336.38-803700.2 & $\mathrm{T} 8$ & 26 & $17.28 \pm 0.14$ & 45 & $13.64 \pm 0.03$ & $2.65 \pm 0.07$ & $3.64 \pm 0.14$ \\
\hline \multicolumn{10}{|c|}{ T9-T9.5 Dwarfs } \\
\hline WISE J000517.48+373720.5 & 2 & WISE J000517.48+373720.5 & T9 & 2 & - & - & $13.27 \pm 0.03$ & $3.61 \pm 0.12$ & - \\
\hline WISE J003829.05+275852.1 & 2 & WISE J003829.05+275852.1 & T9 & 2 & $18.92 \pm 0.04$ & 45 & $14.38 \pm 0.05$ & $3.67 \pm 0.29$ & $4.54 \pm 0.06$ \\
\hline WISEPC J014807.25-720258.7 & 1,36 & WISE J014807.25-720258.7 & T9.5 & 1 & $19.22 \pm 0.04$ & 1 & $14.69 \pm 0.05$ & $>4.25$ & $\begin{array}{l}4.54 \pm 0.07 \\
4.53 \pm 0.07\end{array}$ \\
\hline WISE J032517.69-385454.1 & 2 & WISE J032517.69-385454.1 & T9 & 2 & - & - & $14.98 \pm 0.06$ & $3.47 \pm 0.31$ & - \\
\hline WISE J033515.01+431045.1 & 2 & WISE J033515.01+431045.1 & T9:: & 2 & $19.60 \pm 0.26$ & 45 & $14.60 \pm 0.08$ & $>3.55$ & $5.00 \pm 0.27$ \\
\hline WISE J041358.14-475039.3 & 2 & WISE J041358.14-475039.3 & T9 & 2 & $20.12 \pm 0.20$ & 2 & $15.69 \pm 0.08$ & $>3.70$ & - \\
\hline WISEPA J045853.89+643452.9B & 3 & blended $\mathrm{w} /$ component $\mathrm{A}$ & T9.5 & 61 & - & - & - & - & - \\
\hline UGPS J072227.51-054031.2 & 43 & WISE J072227.27-054029.9 & T9 & 36 & $16.15 \pm 0.21$ & 57 & $12.21 \pm 0.03$ & $2.97 \pm 0.06$ & $3.93 \pm 0.21$ \\
\hline WISE J072312.44+340313.5 & 2 & WISE J072312.44+340313.5 & T9: & 2 & $>18.47$ & 2 & $14.73 \pm 0.08$ & $>3.54$ & $>3.74$ \\
\hline WISEPA J075108.79-763449.6 & 1 & WISE J075108.80-763449.5 & T9 & 1 & $>19.02$ & 1 & $14.52 \pm 0.04$ & $3.01 \pm 0.14$ & $>4.50$ \\
\hline WISE J081117.81-805141.3 & 2 & WISE J081117.81-805141.3 & T9.5: & 2 & $19.88 \pm 0.21$ & 2 & $14.38 \pm 0.04$ & $2.91 \pm 0.13$ & - \\
\hline WISE J105130.01-213859.7 & 2 & WISE J105130.01-213859.7 & T9: & 2 & $19.19 \pm 0.39$ & 2 & $14.54 \pm 0.06$ & $2.63 \pm 0.18$ & - \\
\hline WISEPC J121756.91+162640.2 & 1 & WISE J121756.90+162640.8 & T9 & 1 & $18.18 \pm 0.05$ & 1 & $13.09 \pm 0.03$ & $3.71 \pm 0.14$ & $5.09 \pm 0.06$ \\
\hline WISP J1305-2538 & 68 & $\begin{array}{l}\text { not detected } \\
\text { nof }\end{array}$ & T9.5+ & 68 & $10.10+0.00$ & - & 10.090 .00 & $0.11 \pm 0.14$ & $0.09 \pm 0.00$ \\
\hline WISEPC J131106.24+012252.4 & 1 & WISE J131106.20+012254.3 & T9: & 1 & $19.32 \pm 0.23$ & 45 & $14.76 \pm 0.09$ & $3.50 \pm 0.47$ & $4.56 \pm 0.25$ \\
\hline WISE J131833.98-175826.5 & 2 & WISE J131833.98-175826.5 & T9: & 2 & $17.71 \pm 0.23$ & 2 & $14.75 \pm 0.07$ & $3.35 \pm 0.35$ & $2.96 \pm 0.24$ \\
\hline CFBDSIR J145829+101343B & 50 & blended w/ A component & T9.5 & 45 & $22.51 \pm 0.16$ & 66 & - & - & - \\
\hline WISE J154214.00+223005.2 & 2 & WISE J154214.00+223005.2 & T9.5 & 2 & $21.80 \pm 0.80$ & 2 & $15.02 \pm 0.06$ & $>3.86$ & $6.78 \pm 0.80$ \\
\hline WISEPA J161441.45+173936.7 & 1,3 & WISE J161441.46+173935.5 & T9 & 1 & $18.47 \pm 0.22$ & 1 & $14.25 \pm 0.05$ & $>3.65$ & $4.22 \pm 0.22$ \\
\hline WISEPA J174124.26+255319.5 & $1,3,42$ & WISE J174124.25+255319.6 & T9 & 1,42 & $16.63 \pm 0.03$ & 1 & $12.33 \pm 0.03$ & $3.05 \pm 0.06$ & $4.30 \pm 0.04$ \\
\hline WISEPA J180435.40+311706.1 & 1 & WISE J180435.37+311706.4 & T9.5: & 1 & $19.21 \pm 0.11$ & 1 & $14.70 \pm 0.06$ & $>3.94$ & $4.51 \pm 0.13$ \\
\hline WISE J210200.15-442919.5 & 41 & WISE J210200.15-442919.5 & $\begin{array}{c}\text { T9 } \\
\text { T9 }\end{array}$ & 41 & $18.39 \pm 0.12$ & 45 & $14.12 \pm 0.05$ & $2.83 \pm 0.18$ & $4.26 \pm 0.09$ \\
\hline WISEPA J213456.73-713743.6 & 1 & WISE J213456.73-713744.5 & T9p & 1 & $19.81 \pm 0.12$ & 45 & $13.99 \pm 0.05$ & $\begin{array}{l}2.0010 .10 \\
>3.75\end{array}$ & $5.82 \pm 0.13$ \\
\hline WISEPC J232519.54-410534.9 & 1 & WISE J232519.53-410535.0 & T9p & 1 & $19.22 \pm 0.11$ & 1 & $14.13 \pm 0.05$ & $3.47 \pm 0.25$ & $5.09 \pm 0.12$ \\
\hline $\begin{array}{l}\text { WISE J233226.49-432510.6 } \\
\text { W } 326.9\end{array}$ & 41 & WISE J233226.49-432510.6 & T9: & 41 & $19.40 \pm 0.18$ & $\begin{array}{c}1 \\
45\end{array}$ & $\begin{array}{l}14.15 \pm 0.00 \\
14.99 \pm 0.09\end{array}$ & $\begin{array}{l}.41 \pm 0.20 \\
>2.89\end{array}$ & $4.41 \pm 0.20$ \\
\hline WISEPC J234446.25+103415.8 & 1 & WISE J234446.23+103415.6 & T9 & 1 & $19.07 \pm 0.11$ & 1 & $15.11 \pm 0.12$ & $>2.92$ & $3.96 \pm 0.16$ \\
\hline \multicolumn{10}{|c|}{ Y Dwarfs } \\
\hline WISE J014656.66+423410.0 & 2 & WISE J014656.66+423410.0 & Y0 & 2 & $18.71 \pm 0.24$ & 45 & $15.08 \pm 0.07$ & $>3.91$ & $3.63 \pm 0.25$ \\
\hline WISE J035000.32-565830.2 & 45 & WISE J035000.32-565830.2 & Y1 & 45 & $>21.5$ & 45 & $14.73 \pm 0.06$ & $>4.17$ & $>6.8$ \\
\hline WISE J035934.06-540154.6 & 45 & WISE J035934.06-540154.6 & Yo & 45 & $22.20 \pm 0.43$ & 45 & $15.42 \pm 0.07$ & $>3.87$ & $6.78 \pm 0.44$ \\
\hline WISEPA J041022.71+150248.5 & 1,36 & WISE J041022.71+150248.4 & Yo & 36 & $19.05 \pm 0.09$ & 1 & $14.18 \pm 0.06$ & $>4.15$ & $4.87 \pm 0.11$ \\
\hline WISE J053516.80-750024.9 & 45 & WISE J053516.80-750024.9 & $\geq \mathrm{Y} 1$ & 45 & $>21.6$ & 45 & $15.06 \pm 0.07$ & $>3.86$ & $>6.5$ \\
\hline WISE J071322.55-291751.9 & 45 & WISE J071322.55-291751.9 & Yo & 45 & $>19.3$ & - & $14.48 \pm 0.06$ & $>3.87$ & $>4.8$ \\
\hline WISE J073444.02-715744.0 & 45 & WISE J073444.02-715744.0 & Y0 & 45 & - & - & $15.36 \pm 0.06$ & $>4.06$ & - \\
\hline WD $0806-661 \mathrm{~B}$ & 48 & not detected & (Y?) & 49 & - & - & - & - & - \\
\hline WISEPC J140518.40+553421.4 & 1,36 & WISE J140518.39+553421.3 & YOp & 36 & $>20.5$ & 1 & $14.10 \pm 0.04$ & $>4.72$ & $>6.4$ \\
\hline WISEPA J154151.66-225025.2 & 1,36 & WISE J154151.65-225024.9 & Y0.5 & 36 & $>20.2$ & 1 & $14.25 \pm 0.06$ & $2.49 \pm 0.18$ & $>6.0$ \\
\hline WISEPA J173835.53+273258.9 & 1,36 & WISE J173835.53+273259.0 & Yo & 36 & $20.39 \pm 0.33$ & 1 & $14.55 \pm 0.06$ & $>3.86$ & $5.84 \pm 0.34$ \\
\hline WISEPA J182831.08+265037.8 & 1,36 & WISE J182831.08+265037.7 & $>\mathrm{Y} 2$ & 45 & $22.85 \pm 0.24$ & 1 & $14.39 \pm 0.06$ & $>4.08$ & $\begin{array}{l}0.04 \pm 0.04 \\
8.46 \pm 0.25\end{array}$ \\
\hline WISEPC J205628.90+145953.3 & 1,36 & WISE J205628.91+145953.2 & Yo & 36 & $19.62 \pm 0.31$ & 1 & $13.93 \pm 0.05$ & $>4.33$ & $5.69 \pm 0.31$ \\
\hline
\end{tabular}


Table 7 -Continued

\begin{tabular}{|c|c|c|c|c|c|c|c|c|c|}
\hline $\begin{array}{l}\text { Discovery name } \\
\text { (1) }\end{array}$ & $\begin{array}{l}\text { Disc. } \\
\text { ref. } \\
(2)\end{array}$ & $\begin{array}{c}\text { WISE designation }{ }^{\mathrm{a}} \\
\text { (3) }\end{array}$ & $\begin{array}{l}\text { Spec. } \\
\text { type } \\
(4)\end{array}$ & $\begin{array}{l}\text { Type } \\
\text { ref. } \\
(5)\end{array}$ & $\begin{array}{c}\mathrm{H} \\
(\mathrm{mag}) \\
(6)\end{array}$ & $\begin{array}{l}\mathrm{H} \\
\text { ref. } \\
(7)\end{array}$ & $\begin{array}{l}\text { W2 } \\
(\mathrm{mag}) \\
(8)\end{array}$ & $\begin{array}{l}\text { W1-W2 } \\
(\mathrm{mag}) \\
(9)\end{array}$ & $\begin{array}{l}\mathrm{H}-\mathrm{W} 2 \\
(\mathrm{mag}) \\
(10)\end{array}$ \\
\hline WISE J222055.31-362817.4 & 45 & WISE J222055.31-362817.4 & Y0 & 45 & $20.81 \pm 0.30$ & 45 & $14.66 \pm 0.06$ & $>3.99$ & - \\
\hline
\end{tabular}

Note. - Code for references: (1) Kirkpatrick et al. (2011), (2) Mace et al, in prep., (3) Gelino et al. 2011), (4) Burgasser et al. (2004), (5) Burgasser et al. (2006a), (6) Burgasser et al. (2002), (7) Lodieu et al. (2009), (8) Zapatero Osorio et al. (2002), (9) Artigau et al. (2010), (10) Burningham et al. (2010), (11) Burgasser et al. (1999), (12) (19) Kasper et al (2007), (20) Scholz et al. (2003). (21) Burgasser et al. (2003a). (22) Mugrauer et al. (2006). (23) Luhman et al. (2007). (24) Tinnev et al. (2005). (25) Chiu et al (2008), (26) Burgasser et al. (2011). (27) Nakaiima et al. (1995), (28) Lodieu et al. (2007), (29) Scholz (2010), (30) Burgasser et al. (2010), (31) Burgasser et al. (2000b), (32) Chiu et al. (2006), (33) Scholz (2010), (34) Mainzer et al. (2011), (35) Warren et al. (2007), (36) Cushing et al. (2011), (37) Delorme et al. (2008), (38) Burningham et al. (2008), (39) Goldman et al. (2010), (40) Burningham et al. (2009), (41) Tinney et al, in prep., (42) Scholz et al. (2011), (43) Lucas et al. (2010), (44) Delorme et al. (2010), (45) This paper, (46) Revlé et al. (2010), (47) Geißler et al. (2011), (48) Luhman et al. (2011), (49) Luhman et al. (2012), (50) Liu et al. (2011a), (51) Murrav et al. (2011), (52) Liu et al. (2010), (53) Stumpf et al. (2010), (54) Lodieu et al. (2009), (55) Burgess et al. (2009), (56) Albert et al. (2011), (57) 2MASS, (58) UKIDSS, (59) CFBDS, (60) Stumpf et al. (2008), (61) Burgasser et al. (2012), (62) Burgasser et al. (2006b), (63) Matthews et al. (1996), (64) Burgasser et al. (2003b), (65) Dupuv \& Liu (2012), (66) Liu et al. (2011a), (67) Wright et al. (2012), (68) Masters et al. (2012).

"WISE sources are given designations as follows. The prefix is "WISE" for sources taken from the four-band cryogenic atlas source catalog, "WISER" for sources taken from the four-band cryogenic atlas source reject table, "WISEPC" for sources taken from the first-pass precessing operations coadd Source Working Database, or "WISEPA" for objects drawn from the preliminary release Atlas Tile Source Working Database. The suffix is the J2000 position of the source in the format Jhhmmss.ss \pm ddmmss.s.

b Also known as IC348 CH4 $2034449.52+320635.4$

${ }^{\mathrm{c}}$ Also known as HIP $73786 \mathrm{~B}$ or Gl 576B.

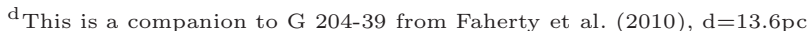

${ }^{e}$ Object is seen on the WISE images but is not successfully extracted.

f Object is not detected by WISE Pass2 processing, so the quoted WISE photometry comes from Pass1.

${ }^{\mathrm{g}}$ These values represent the combined light of the composite system.

${ }^{\mathrm{h}}$ This object may not exist.

Cushing et al 2011) assigns this binary a composite type of T9 on the revised late-T dwarf classification scheme. Given the Dupuy \& Lill 2012 ) measurements of the parallax and the Liu et al. (2011a) $H$-band measurements of the individual components, we infer spectral types of T8.5 and T9.5 based on absolute $H$ magnitudes of 17.8 and 20.2 mag.

${ }^{\mathrm{j}}$ Spitzer/IRAC follow-up of this sources shows that the WISE photometry is blended with a bright, nearby source. 
Table 8. Distances for Objects in Table 7

\begin{tabular}{cccccccc}
\hline \hline Discovery name & Spec. & $\pi_{\text {trig }}$ & $\pi_{\text {trig }}$ & $\mathrm{D}_{H}$ & $\mathrm{D}_{W 2}$ & $\mathrm{D}_{\text {adopted }}$ & $\frac{V}{V_{\max }}$ \\
& type & $(\mathrm{pc})$ & ref. & $(\mathrm{pc})$ & $\begin{array}{c}(\mathrm{pc}) \\
(\mathrm{pc})\end{array}$ & $\begin{array}{c}(7) \\
(1)\end{array}$ \\
& $(2)$ & $(3)$ & $(4)$ & $(5)$ & $(6)$ & $(8)$ & \\
\hline
\end{tabular}

T6-T6.5 Dwarfs (distance limit $\left.=20 \mathrm{pc},\left\langle\frac{V}{\nabla_{\max }}\right\rangle=0.41\right)$

\begin{tabular}{|c|c|c|c|c|c|c|c|}
\hline WISEPC J000849.76-173922.6 & T6 & - & - & 31.5 & 21.8 & 26.7 & - \\
\hline 2MASS J00345157+0523050 & T6.5 & - & - & 9.1 & 8.1 & 8.6 & 0.08 \\
\hline WISE J003830.54+840517.7 & T6 & - & - & - & 31.2 & 31.2 & - \\
\hline WISE J013525.64+171503.4 & T6 & - & - & - & 23.7 & 23.7 & - \\
\hline WISEPA J022105.94+384202.9 & T6.5 & - & - & 22.9 & 22.0 & 22.4 & - \\
\hline CFBDS J022638-072831 & T6.5 & - & - & 39.8 & - & 39.8 & - \\
\hline 2MASSI J0243137-245329 & T6 & $0.0936 \pm 0.0036$ & 5 & 9.4 & 10.2 & 10.7 & 0.15 \\
\hline CFBDS J025401-182529 & T6.5 & - & - & 37.0 & 35.2 & 36.1 & - \\
\hline UGCS J030013.86+490142.5 & T6.5 & - & - & 35.0 & 38.4 & 36.7 & - \\
\hline CFBDS J030130-104504 & T6 & - & - & - & 30.9 & 30.9 & - \\
\hline CFBDS J030225-144125 & T6 & - & - & 34.6 & - & 34.6 & - \\
\hline WISEPA J030533.54+395434.4 & T6 & - & - & 22.9 & 23.1 & 23.0 & - \\
\hline WISEPA J030724.57+290447.6 & T6.5 & - & - & 25.7 & 25.1 & 25.4 & - \\
\hline CFHT J0344+3206 & (T6:) & - & - & 188.3 & - & 188.3 & - \\
\hline WISEPA J041054.48+141131.6 & T6 & - & - & 24.9 & 27.7 & 26.3 & - \\
\hline WISEPA J051317.28+060814.7 & T6.5 & - & - & 12.5 & 14.9 & 13.7 & 0.32 \\
\hline WISEPA J052536.33+673952.3 & T6p & - & - & 33.0 & 27.2 & 30.1 & - \\
\hline SOri70 & T6: & - & - & 106.8 & - & 106.8 & - \\
\hline WISEPA J054231.26-162829.1 & T6.5 & - & - & 15.3 & 15.8 & 15.5 & 0.47 \\
\hline WISEPA J061213.93-303612.7 & T6 & - & - & 22.7 & 17.1 & 19.9 & 0.99 \\
\hline WISEPA J061407.49+391236.4 & T6 & - & - & 16.5 & 14.3 & 15.4 & 0.46 \\
\hline WISEPA J062542.21+564625.5 & T6 & - & - & 21.1 & 20.0 & 20.6 & - \\
\hline WISEPA J062720.07-111428.8 & T6 & - & - & 10.8 & 12.0 & 11.4 & 0.19 \\
\hline WISE J073347.94+754439.2 & T6 & - & - & - & 24.0 & 24.0 & - \\
\hline DENIS J081730.0-615520 & $\mathrm{T} 6$ & $0.203 \pm 0.013$ & 3 & 4.5 & 4.7 & 4.9 & 0.01 \\
\hline ULAS J081948.09+073323.3 & T6p & - & - & 41.4 & - & 41.4 & - \\
\hline ULAS J085342.94+000651.8 & T6p & - & - & 61.2 & 38.4 & 49.8 & - \\
\hline ULAS J085715.96+091325.3 & T6 & - & - & 52.8 & - & 52.8 & - \\
\hline WISEPC J092906.77+040957.9 & T6.5 & - & - & 22.1 & 17.7 & 19.9 & 0.99 \\
\hline 2MASSI J0937347+293142 & T6p & $0.1634 \pm 0.0018$ & 7 & 7.7 & 5.7 & 6.1 & 0.03 \\
\hline WISEPC J095259.29+195507.3 & T6 & - & - & 24.5 & 19.7 & 22.1 & - \\
\hline WISEPA J101905.63+652954.2 & T6 & - & - & 17.7 & 17.1 & 17.4 & 0.66 \\
\hline ULAS J103434.52-001553.0 & T6.5p & - & - & 46.8 & - & 46.8 & - \\
\hline CFBDS J104209+580856 & T6.5 & - & - & 32.5 & 29.0 & 30.7 & - \\
\hline 2MASSI J1047538+212423 & T6.5 & $0.0947 \pm 0.0038$ & 5 & 10.7 & 9.9 & 10.6 & 0.15 \\
\hline WISEPC J112254.73+255021.5 & T6 & - & - & 18.7 & 16.6 & 17.7 & 0.69 \\
\hline ULAS J115038.79+094942.9 & T6.5p & $0.0168 \pm 0.0075$ & 1 & 52.0 & - & 59.5 & - \\
\hline ULAS J115338.74-014724.1 & T6 & - & - & 34.6 & - & 34.6 & - \\
\hline NTTDF $1205-0744$ & T6: & - & - & - & - & $\sim 90^{\mathrm{b}}$ & - \\
\hline ULAS J120744.65+133902.7 & $\mathrm{T} 6$ & - & - & 44.5 & 38.4 & 41.5 & - \\
\hline 2MASS J12095613-1004008B & T6.5: & $0.0307 \pm 0.013$ & 11 & 30.6 & - & 32.6 & - \\
\hline SDSS J121440.95+631643.4B & (T6) & - & - & - & - & $>20.9^{c}$ & - \\
\hline WISE J122558.86-101345.0 & T6 & - & - & 17.2 & 16.8 & 17.0 & 0.61 \\
\hline 2MASS J12373919+6526148 & T6.5 & $0.0961 \pm 0.0048$ & 5 & 10.4 & 9.8 & 10.4 & 0.14 \\
\hline WISEPC J132004.16+603426.2 & T6.5 & - & - & 15.1 & 19.2 & 17.2 & 0.64 \\
\hline ULAS J132605.18+120009.9 & T6p & - & - & 33.9 & 31.8 & 32.9 & - \\
\hline SDSSp J134646.45-003150.4 & T6.5 & $0.0683 \pm 0.0023$ & 9 & 9.2 & 13.1 & 14.6 & 0.39 \\
\hline ULAS J144555.24+125735.1 & T6.5 & - & - & 49.0 & - & 49.0 & - \\
\hline HIP 73786B (J1504+0538) & T6:p & $0.0538 \pm 0.0028$ & 6 & 22.6 & 18.7 & 18.6 & 0.80 \\
\hline WISEPC J150649.97+702736.0 & T6 & $0.193 \pm 0.026$ & 14 & 5.3 & 4.8 & 5.2 & 0.02 \\
\hline ULAS J152526.25+095814.3 & T6.5 & - & - & 50.6 & 49.0 & 49.8 & - \\
\hline ULAS J152912.23+092228.5 & T6 & - & - & 59.0 & - & 59.0 & - \\
\hline 2MASSI J1553022+153236A & T6.5 & $0.0751 \pm 0.0009$ & 11 & 13.7 & $>10.1$ & 13.3 & 0.29 \\
\hline WISEPA J161215.94-342027.1 & T6.5 & - & - & 18.3 & 15.6 & 17.0 & 0.61 \\
\hline 2MASS J16150413+1340079 & T6 & - & - & 17.5 & 18.4 & 17.9 & 0.72 \\
\hline WISEPA J162208.94-095934.6 & T6 & - & - & 14.3 & 18.2 & 16.2 & 0.53 \\
\hline SDSSp J162414.37+002915.6 & T6 & $0.0909 \pm 0.0012$ & 9 & 11.2 & 11.1 & 11.0 & 0.17 \\
\hline WISEPA J162725.64+325525.5 & $\mathrm{T} 6$ & - & - & 16.8 & 14.0 & 15.4 & 0.46 \\
\hline WISE J172134.46+111739.4 & T6 & - & - & - & 19.1 & 19.1 & 0.87 \\
\hline WISEPA J172844.93+571643.6 & T6 & - & - & 33.2 & 26.8 & 30.0 & - \\
\hline WISE J174640.78-033818.0 & T6 & - & - & 27.2 & 24.2 & 25.7 & - \\
\hline
\end{tabular}


Table 8-Continued

\begin{tabular}{|c|c|c|c|c|c|c|c|}
\hline $\begin{array}{c}\text { Discovery name } \\
\text { (1) }\end{array}$ & $\begin{array}{l}\text { Spec. } \\
\text { type } \\
(2)\end{array}$ & $\begin{array}{l}\pi_{\text {trig }} \\
(\mathrm{pc}) \\
(3)\end{array}$ & $\begin{array}{l}\pi_{t r i g} \\
\text { ref. } \\
(4)\end{array}$ & $\begin{array}{l}\mathrm{D}_{H} \\
\text { (pc) } \\
(5)\end{array}$ & $\begin{array}{c}\mathrm{D}_{W 2} \\
(\mathrm{pc}) \\
(6)\end{array}$ & $\begin{array}{l}\text { Dadopted } \\
\text { (pc) } \\
(7)\end{array}$ & $\begin{array}{c}\frac{V}{V_{\max }} \\
(8)\end{array}$ \\
\hline SDSS J175805.46+463311.9 & T6.5 & $0.0710 \pm 0.0019$ & 6 & 13.2 & 14.7 & 13.9 & 0.34 \\
\hline SCR J1845-6357B & T6 & $0.2595 \pm 0.0011$ & 10 & - & - & 3.9 & 0.01 \\
\hline WISEPA J190624.75+450808.2 & $\mathrm{T} 6$ & - & - & 16.2 & 15.5 & 15.8 & 0.49 \\
\hline WISE J192841.35+235604.9 & $\mathrm{T} 6$ & - & - & - & 7.0 & 7.0 & 0.04 \\
\hline$\epsilon$ Ind $\mathrm{Bb}$ & $\mathrm{T} 6$ & $0.2761 \pm 0.0003$ & 6 & - & - & 3.6 & 0.01 \\
\hline 2MASS J21543318+5942187 & T6 & - & - & 12.6 & 13.4 & 13.0 & 0.27 \\
\hline UDXS J221903.10+002418.2 & $(\mathrm{T} 6:)$ & - & - & - & - & $\sim 60^{\mathrm{d}}$ & - \\
\hline 2MASS J22282889-4310262 & T6 & - & - & 10.4 & 12.4 & 11.4 & 0.19 \\
\hline WISE J223720.39+722833.8 & $\mathrm{T} 6$ & - & - & - & 14.1 & 14.1 & 0.35 \\
\hline WISE J230133.32+021635.1 & $\mathrm{T} 6$ & - & - & - & 18.6 & 18.6 & 0.80 \\
\hline ULAS J230601.02+130225.0 & T6.5 & - & - & 29.5 & 26.1 & 27.8 & - \\
\hline ULAS J231557.61+132256.2 & $\mathrm{T} 6.5$ & - & - & 31.8 & 31.3 & 31.6 & - \\
\hline CFBDS J232304-015232 & T6 & - & - & 27.3 & 27.5 & 27.4 & - \\
\hline WISE J234228.98+085620.2 & $\mathrm{T} 6.5$ & - & - & - & 15.7 & 15.7 & 0.48 \\
\hline WISEPA J234351.20-741847.0 & T6 & - & - & $>14.9$ & 14.9 & 14.9 & 0.41 \\
\hline WISE J235716.49+122741.8 & $\mathrm{T} 6$ & - & - & - & 16.8 & 16.8 & 0.59 \\
\hline \multicolumn{8}{|c|}{$\mathrm{T} 7-\mathrm{T} 7.5$ Dwarfs $\left(\right.$ distance limit $\left.=20 \mathrm{pc},\left\langle\frac{V}{V_{\max }}\right\rangle=0.37\right)$} \\
\hline HD 3651B & $\mathrm{T} 7.5$ & $0.0904 \pm 0.0003$ & 6 & 11.0 & - & 11.1 & 0.17 \\
\hline WISE J004024.88+090054.8 & $\mathrm{T} 7$ & - & - & - & 14.6 & 14.6 & 0.39 \\
\hline 2MASS J00501994-3322402 & $\mathrm{T} 7$ & - & - & 9.0 & 12.3 & 10.7 & 0.15 \\
\hline WISEPA J012333.21+414203.9 & $\mathrm{T} 7$ & - & - & 16.9 & 24.4 & 20.6 & - \\
\hline ULAS J013939.77+004813.8 & $\mathrm{T} 7.5$ & - & - & 32.7 & - & 32.7 & - \\
\hline ULAS J015024.37+135924.0 & $\mathrm{T} 7.5$ & - & - & 20.6 & 25.4 & 23.0 & - \\
\hline WISEPC J022322.39-293258.1 & $\mathrm{T} 7.5$ & - & - & 14.2 & 14.6 & 14.4 & 0.37 \\
\hline WISEPA J022623.98-021142.8 & T7 & - & - & 36.0 & 19.9 & 27.9 & - \\
\hline WISE J024124.73-365328.0 & $\mathrm{T} 7$ & - & - & 15.3 & 17.7 & 16.5 & 0.56 \\
\hline CFBDS J030135-161418 & $\mathrm{T} 7$ & - & - & 38.5 & 31.6 & 35.0 & - \\
\hline WISE J032547.72+083118.2 & $\mathrm{T} 7$ & - & - & 10.4 & 12.0 & 11.2 & 0.18 \\
\hline 2MASS J03480772-602227 & $\mathrm{T} 7$ & - & - & 7.9 & 7.7 & 7.8 & 0.06 \\
\hline WISEPA J052844.51-330823.9 & $\mathrm{T} 7 \mathrm{p}$ & - & - & 15.2 & 20.0 & 17.6 & 0.68 \\
\hline UGCS J053022.52-052447.4 & T7: & - & - & 41.1 & - & 41.1 & - \\
\hline Gl229B & $\mathrm{T} 7 \mathrm{p}$ & $0.1738 \pm 0.0010$ & 6 & 4.4 & - & 5.8 & 0.02 \\
\hline WISE J061437.73+095135.0 & $\mathrm{T} 7$ & - & - & 14.2 & 17.3 & 15.8 & 0.49 \\
\hline 2MASSI J0727182+171001 & $\mathrm{T} 7$ & $0.1101 \pm 0.0023$ & 5 & 8.7 & 9.4 & 9.1 & 0.09 \\
\hline ULAS J085910.69+101017.1 & $\mathrm{T} 7$ & - & - & 31.9 & 27.4 & 29.6 & - \\
\hline ULAS J090116.23-030635.0 & $\mathrm{T} 7.5$ & $0.0626 \pm 0.0026$ & 1 & 24.2 & 18.9 & 16.0 & 0.51 \\
\hline CFBDS J092250+152741 & $\mathrm{T} 7$ & - & - & 35.5 & - & 35.5 & - \\
\hline ULAS J094806.06+064805.0 & $\mathrm{T} 7$ & $0.0272 \pm 0.0042$ & 1 & 47.8 & - & 36.8 & - \\
\hline WISE J103907.73-160002.9 & $\mathrm{T} 7.5$ & - & - & 13.5 & 15.5 & 14.5 & 0.38 \\
\hline 2MASS J11145133-2618235 & $\mathrm{T} 7.5$ & - & - & 6.9 & 6.4 & 6.6 & 0.04 \\
\hline WISE J112438.12-042149.7 & $\mathrm{T} 7$ & - & - & - & 15.5 & 15.5 & 0.47 \\
\hline 2MASSI J1217110-031113 & $\mathrm{T} 7.5$ & $0.0908 \pm 0.0022$ & 9 & 6.9 & 9.9 & 11.0 & 0.17 \\
\hline WISP J1232-0033 & $\mathrm{T} 7$ & - & - & - & - & $\sim 270^{\mathrm{i}}$ & - \\
\hline ULAS J124804.56+075904.0 & $\mathrm{T} 7$ & - & - & 26.2 & 28.5 & 27.3 & - \\
\hline Kelu-1 $\mathrm{Ab}^{\mathrm{a}}$ & $(\mathrm{T} 7.5)$ & $0.0536 \pm 0.0020$ & 8 & - & - & 18.7 & 0.82 \\
\hline ULAS J131508.42+082627.4 & $\mathrm{T} 7.5$ & $0.0428 \pm 0.0077$ & 1 & 39.0 & - & 23.4 & - \\
\hline ULAS J134940.81+091833.3 & $\mathrm{T} 7$ & - & - & 47.2 & - & 47.2 & - \\
\hline ULAS J141623.94+134836.3 & $\mathrm{T} 7.5$ & $0.1099 \pm 0.0018$ & 11 & 16.1 & 8.2 & 9.1 & 0.09 \\
\hline WISEPC J145715.03+581510.2 & $\mathrm{T} 7$ & - & - & 13.1 & 18.8 & 16.0 & 0.51 \\
\hline G1570D & $\mathrm{T} 7.5$ & $0.1712 \pm 0.0009$ & 6 & 5.6 & 6.0 & 5.8 & 0.02 \\
\hline SDSS J150411.63+102718.4 & $\mathrm{T} 7$ & $0.0461 \pm 0.0015$ & 11 & - & 15.6 & 21.7 & - \\
\hline WISE J152305.10+312537.6 & $\mathrm{T} 7 \mathrm{p}$ & - & - & 33.6 & 18.2 & 25.9 & - \\
\hline WISE J154459.27+584204.5 & $\mathrm{T} 7.5$ & - & - & 22.9 & 23.1 & 23.0 & - \\
\hline 2MASSI J1553022+153236B & $\mathrm{T} 7.5$ & $0.0751 \pm 0.0009$ & 11 & 10.8 & - & 13.3 & 0.29 \\
\hline SDSS J162838.77+230821.1 & $\mathrm{T} 7$ & $0.0751 \pm 0.0009$ & 11 & 10.2 & 14.9 & 13.3 & 0.29 \\
\hline WISEPA J185215.78+353716.3 & $\mathrm{T} 7$ & - & - & $>18.6$ & 17.0 & 17.0 & 0.61 \\
\hline WISE J201404.13+042408.5 & $\mathrm{T} 7 \mathrm{p}$ & - & - & 33.1 & 25.9 & 29.5 & - \\
\hline WISEPA J201824.96-742325.9 & $\mathrm{T} 7$ & - & - & 16.7 & 12.7 & 14.7 & 0.40 \\
\hline WISEPC J215751.38+265931.4 & $\mathrm{T} 7$ & - & - & 19.0 & 18.9 & 18.9 & 0.84 \\
\hline WISEPC J220922.10-273439.5 & $\mathrm{T} 7$ & - & - & $>11.7$ & 14.1 & 14.1 & 0.35 \\
\hline WISEPC J221354.69+091139.4 & $\mathrm{T} 7$ & - & - & 16.1 & 20.1 & 18.0 & 0.73 \\
\hline UDXS J221611.51+003308.1 & (T7.5:) & - & - & - & - & $\sim 81^{\mathrm{d}}$ & - \\
\hline
\end{tabular}


Table 8-Continued

\begin{tabular}{|c|c|c|c|c|c|c|c|}
\hline $\begin{array}{c}\text { Discovery name } \\
\text { (1) }\end{array}$ & $\begin{array}{l}\text { Spec. } \\
\text { type } \\
(2)\end{array}$ & $\begin{array}{l}\pi_{\text {trig }} \\
\text { (pc) } \\
(3)\end{array}$ & $\begin{array}{l}\pi_{\text {trig }} \\
\text { ref. } \\
(4)\end{array}$ & $\begin{array}{l}\mathrm{D}_{H} \\
(\mathrm{pc}) \\
(5)\end{array}$ & $\begin{array}{c}\mathrm{D}_{W 2} \\
(\mathrm{pc}) \\
(6)\end{array}$ & $\begin{array}{l}\text { Dadopted } \\
\quad(\mathrm{pc}) \\
\quad(7)\end{array}$ & $\begin{array}{c}\frac{V}{\nabla_{\max }} \\
(8)\end{array}$ \\
\hline WISEPC J231939.13-184404.3 & $\mathrm{T} 7.5$ & - & - & 19.1 & 12.9 & 16.0 & 0.51 \\
\hline ULAS J232123.79+135454.9 & $\mathrm{T} 7.5$ & - & - & 13.2 & 15.0 & 14.1 & 0.35 \\
\hline ULAS J232802.03+134544.8 & $\mathrm{T} 7$ & - & - & 26.4 & 28.8 & 27.6 & - \\
\hline WISE J233543.79+422255.2 & $\mathrm{T} 7$ & - & - & - & 27.7 & 27.7 & - \\
\hline WISEPC J234026.62-074507.2 & $\mathrm{T} 7$ & - & - & 10.6 & 12.5 & 11.6 & 0.20 \\
\hline WISEPC J234841.10-102844.4 & $\mathrm{T} 7$ & - & - & 14.9 & 18.2 & 16.6 & 0.57 \\
\hline \multicolumn{8}{|c|}{ T8-T8.5 Dwarfs (distance limit $\left.=20 \mathrm{pc},\left\langle\frac{V}{V \max }\right\rangle=0.35\right)$} \\
\hline WISE J001505.87-461517.6 & T8 & - & - & - & 14.9 & 14.9 & 0.41 \\
\hline WISE J003231.09-494651.4 & $\mathrm{T} 8.5$ & - & - & - & 19.3 & 19.3 & 0.90 \\
\hline ULAS J003402.77-005206.7 & $\mathrm{T} 8.5$ & $0.0780 \pm 0.0036$ & 1 & 13.4 & 14.9 & 12.8 & 0.26 \\
\hline WISE J004945.61+215120.0 & $\mathrm{T} 8.5$ & - & - & - & 7.4 & 7.4 & 0.05 \\
\hline CFBDS J005910.90-011401.3 & $\mathrm{T} 8.5$ & $0.1011 \pm 0.0025$ & 11 & 12.1 & 10.2 & 9.9 & 0.12 \\
\hline CFBDS J013302+023128 & $\mathrm{T} 8.5$ & - & - & 14.2 & 19.6 & 16.9 & 0.60 \\
\hline WISE J024512.62-345047.8 & T8 & - & - & - & 17.2 & 17.2 & 0.64 \\
\hline WISE J024714.52+372523.5 & $\mathrm{T} 8$ & - & - & 16.7 & 18.3 & 17.5 & 0.67 \\
\hline WISEPA J025409.45+022359.1 & $\mathrm{T} 8$ & $0.166 \pm 0.026$ & 14 & 6.8 & 7.4 & 6.0 & 0.03 \\
\hline WISEPA J031325.96+780744.2 & $\mathrm{T} 8.5$ & - & - & 9.0 & 8.1 & 8.6 & 0.08 \\
\hline WISE J031624.35+430709.1 & T8 & - & - & 32.6 & 17.2 & $32.6^{\mathrm{h}}$ & - \\
\hline WISE J032120.91-734758.8 & $\mathrm{T} 8$ & - & - & 24.3 & 27.7 & 26.0 & - \\
\hline WISEPC J032337.53-602554.9 & $\mathrm{T} 8.5$ & - & - & 12.8 & 14.9 & 13.9 & 0.34 \\
\hline WISE J033605.05-014350.4 & T8:: & - & - & - & 17.0 & 17.0 & 0.61 \\
\hline 2MASSW J0337036-175807B & (T8??) & - & - & - & - & $\sim 29^{\mathrm{e}}$ & - \\
\hline 2MASSI J0415195-093506 & T8 & $0.1773 \pm 0.0022$ & 11 & 4.8 & 6.0 & 5.6 & 0.02 \\
\hline WISEPA J045853.89+643452.9A & $\mathrm{T} 8.5$ & - & - & $>8.1$ & $>7.6$ & $\sim 11^{\mathrm{f}}$ & 0.17 \\
\hline WISEPA J050003.05-122343.2 & T8 & - & - & 15.8 & 12.9 & 14.4 & 0.37 \\
\hline WISEPA J062309.94-045624.6 & $\mathrm{T} 8$ & - & - & 10.9 & 12.2 & 11.5 & 0.19 \\
\hline 2MASS J07290002-3954043 & $\mathrm{T} 8 \mathrm{p}$ & - & - & 5.9 & 8.2 & 7.1 & 0.04 \\
\hline WISEPA J074457.15+562821.8 & T8 & - & - & 12.3 & 16.6 & 14.5 & 0.38 \\
\hline WISEPA J075003.84+272544.8 & $\mathrm{T} 8.5$ & - & - & 16.9 & 14.6 & 15.8 & 0.49 \\
\hline WISEPC J075946.98-490454.0 & T8 & - & - & 11.4 & 12.4 & 11.9 & 0.21 \\
\hline WISE J081220.04+402106.2 & $\mathrm{T} 8$ & - & - & 17.1 & 21.8 & 19.5 & 0.93 \\
\hline WISEPC J083641.12-185947.2 & $\mathrm{T} 8 \mathrm{p}$ & - & - & 21.5 & 22.8 & 22.2 & - \\
\hline WISEPA J085716.25+560407.6 & $\mathrm{T} 8$ & - & - & 11.8 & 14.0 & 12.9 & 0.27 \\
\hline WISEPA J090649.36+473538.6 & $\mathrm{T} 8$ & - & - & 13.7 & 17.9 & 15.8 & 0.49 \\
\hline 2MASS J09393548-2448279 & $\mathrm{T} 8$ & $0.1873 \pm 0.0046$ & 15 & 5.4 & 4.5 & 5.3 & 0.02 \\
\hline ULAS J101721.40+011817.9 & $\mathrm{T} 8 \mathrm{p}$ & - & - & 24.4 & - & 24.4 & - \\
\hline WISEPC J101808.05-244557.7 & $\mathrm{T} 8$ & - & - & 14.9 & 14.4 & 14.6 & 0.39 \\
\hline CFBDS J102841+565401 & T8 & - & - & 17.8 & - & 17.8 & 0.70 \\
\hline WISEPC J104245.23-384238.3 & $\mathrm{T} 8.5$ & - & - & 17.6 & 15.0 & 16.3 & 0.54 \\
\hline$\xi \mathrm{UMaC}$ & $\mathrm{T} 8.5$ & $0.1132 \pm 0.0046$ & 18 & 11.4 & 8.6 & 8.8 & 0.09 \\
\hline WISEPC J115013.88+630240.7 & T8 & - & - & $>15.0$ & 10.1 & 10.1 & 0.13 \\
\hline 2MASS J12255432-2739466B & $\mathrm{T} 8$ & $0.0751 \pm 0.0025$ & 9 & 9.0 & - & 13.3 & 0.29 \\
\hline ULAS J123828.51+095351.3 & $\mathrm{T} 8$ & - & - & 25.9 & 23.3 & 24.6 & - \\
\hline Ross $458 \mathrm{C}$ & $\mathrm{T} 8$ & $0.0855 \pm 0.0015$ & 6 & 9.5 & 11.8 & 11.7 & 0.20 \\
\hline ULAS J130217.21+130851.2 & T8 & - & - & 19.7 & 20.6 & 20.1 & - \\
\hline WISEPA J132233.66-234017.1 & $\mathrm{T} 8$ & - & - & 7.9 & 12.8 & 10.4 & 0.14 \\
\hline ULAS J133553.45+113005.2 & $\mathrm{T} 8.5$ & $0.0967 \pm 0.0032$ & 1 & 12.0 & 11.1 & 10.3 & 0.14 \\
\hline WISE J143311.42-083736.4 & T8:: & - & - & 28.7 & 23.4 & 26.0 & - \\
\hline WISEPA J143602.19-181421.8 & $\mathrm{T} 8 \mathrm{p}$ & - & - & $>12.5$ & 18.4 & 18.4 & 0.78 \\
\hline CFBDSIR J145829+101343A & $\mathrm{T} 8.5$ & $0.0340 \pm 0.0026$ & 11 & 29.2 & $>25.4$ & 29.4 & - \\
\hline WISE J151721.13+052929.3 & T8 & - & - & 22.1 & 22.3 & 22.2 & - \\
\hline WISEPC J151906.64+700931.5 & $\mathrm{T} 8$ & - & - & 17.0 & 14.2 & 15.6 & 0.47 \\
\hline WISEPA J161705.75+180714.3 & $\mathrm{T} 8$ & - & - & 16.6 & 14.3 & 15.4 & 0.46 \\
\hline WISEPA J165311.05+444423.9 & $\mathrm{T} 8$ & - & - & 12.0 & 12.2 & 12.1 & 0.22 \\
\hline WISEPA J171104.60+350036.8 & $\mathrm{T} 8$ & - & - & $>15.8$ & 18.5 & 18.5 & 0.79 \\
\hline WISEPA J171717.02+612859.3 & $\mathrm{T} 8$ & - & - & 22.7 & 21.9 & 22.3 & - \\
\hline WISE J180901.07+383805.4 & T8 & - & - & - & 22.8 & 22.8 & - \\
\hline WISEPA J181210.85+272144.3 & $\mathrm{T} 8.5$ : & - & - & 15.7 & 13.1 & 14.4 & 0.37 \\
\hline WISEPA J195905.66-333833.7 & T8 & - & - & 10.2 & 12.6 & 11.4 & 0.19 \\
\hline WISEPC J201546.27+664645.1 & $\mathrm{T} 8$ & - & - & 12.8 & 18.1 & 15.5 & 0.47 \\
\hline WISE J201920.76-114807.6 & $\mathrm{T} 8.5$ : & - & - & 12.4 & 13.7 & 13.0 & 0.27 \\
\hline Wolf $940 \mathrm{~B}$ & $\mathrm{~T} 8.5$ & $0.0835 \pm 0.0039$ & 17 & 15.2 & 13.2 & 12.0 & 0.22 \\
\hline
\end{tabular}


Table 8-Continued

\begin{tabular}{|c|c|c|c|c|c|c|c|}
\hline $\begin{array}{c}\text { Discovery name } \\
\text { (1) }\end{array}$ & $\begin{array}{l}\text { Spec. } \\
\text { type } \\
(2)\end{array}$ & $\begin{array}{l}\pi_{\text {trig }} \\
(\mathrm{pc}) \\
(3)\end{array}$ & $\begin{array}{l}\pi_{\text {trig }} \\
\text { ref. } \\
(4)\end{array}$ & $\begin{array}{l}\mathrm{D}_{H} \\
\text { (pc) } \\
\text { (5) }\end{array}$ & $\begin{array}{l}\mathrm{D}_{W 2} \\
(\mathrm{pc}) \\
(6)\end{array}$ & $\begin{array}{l}\mathrm{D}_{\text {adopted }} \\
\text { (pc) } \\
\text { (7) }\end{array}$ & $\begin{array}{c}\frac{V}{\max } \\
(8)\end{array}$ \\
\hline WISEPC J222623.05+044003.9 & $\mathrm{T} 8$ & - & - & 9.2 & 18.4 & 13.8 & 0.33 \\
\hline WISEPC J225540.74-311841.8 & T8 & - & - & 13.0 & 14.4 & 13.7 & 0.32 \\
\hline WISEPA J231336.40-803700.3 & $\mathrm{T} 8$ & - & - & 10.7 & 11.2 & 11.0 & 0.17 \\
\hline \multicolumn{8}{|c|}{ T9-T9.5 Dwarfs (distance limit $\left.=15 \mathrm{pc},\left\langle\frac{V}{\frac{\max }{2}}\right\rangle=0.44\right)$} \\
\hline WISE J000517.48+373720.5 & T9 & - & - & - & 7.1 & 7.1 & 0.11 \\
\hline WISE J003829.05+275852.1 & T9 & - & - & 10.8 & 11.9 & 11.3 & 0.43 \\
\hline WISEPC J014807.25-720258.7 & T9.5 & - & - & 7.5 & 10.8 & 9.2 & 0.23 \\
\hline WISE J032517.69-385454.1 & T9 & - & - & - & 15.7 & 15.7 & - \\
\hline WISE J033515.01+431045.1 & T9:: & - & - & 14.8 & 13.2 & 14.0 & 0.81 \\
\hline WISE J041358.14-475039.3 & T9 & - & - & 18.8 & 21.7 & 20.3 & - \\
\hline WISEPA J045853.89+643452.9B & T9.5 & - & - & - & - & $\sim 11^{\mathrm{f}}$ & 0.39 \\
\hline UGPS J072227.51-054031.2 & T9 & $0.246 \pm 0.033$ & 2 & 3.0 & 4.4 & 4.1 & 0.02 \\
\hline WISE J072312.44+340313.5 & T9: & - & - & $>8.8$ & 14.0 & 14.0 & 0.81 \\
\hline WISEPA J075108.79-763449.6 & T9 & - & - & $>11.3$ & 12.7 & 12.0 & 0.51 \\
\hline WISE J081117.81-805141.3 & T9.5: & - & - & 10.2 & 9.4 & 9.8 & 0.28 \\
\hline WISE J105130.01-213859.7 & T9: & - & - & 12.2 & 12.8 & 12.5 & 0.58 \\
\hline WISEPC J121756.91+162640.2 & T9 & - & - & 7.7 & 6.6 & 7.1 & 0.11 \\
\hline WISP J1305-2538 & $\mathrm{T} 9.5+$ & - & - & - & - & $\sim 50^{\mathrm{i}}$ & - \\
\hline WISEPC J131106.24+012252.4 & T9: & - & - & 13.0 & 14.2 & 13.6 & 0.75 \\
\hline WISE J131833.98-175826.5 & T9: & - & - & 6.2 & 14.1 & 10.1 & 0.31 \\
\hline CFBDSIR J145829+101343B & T9.5 & $0.0340 \pm 0.0026$ & 11 & 34.1 & - & 29.4 & - \\
\hline WISE J154214.00+223005.2 & T9.5 & - & - & 24.6: & 12.6 & $12.6^{\mathrm{g}}$ & 0.59 \\
\hline WISEPA J161441.45+173936.7 & T9 & - & - & 8.8 & 11.2 & 10.0 & 0.30 \\
\hline WISEPA J174124.26+255319.5 & T9 & $0.176 \pm 0.022$ & 14 & 3.8 & 4.6 & 5.7 & 0.05 \\
\hline WISEPA J180435.40+311706.1 & T9.5: & - & - & 7.5 & 10.9 & 9.2 & 0.23 \\
\hline WISE J210200.15-442919.5 & T9 & - & - & 8.5 & 10.5 & 9.5 & 0.25 \\
\hline WISEPA J213456.73-713743.6 & T9p & - & - & 16.3 & 9.9 & 13.1 & 0.67 \\
\hline WISEPC J232519.54-410534.9 & T9p & - & - & 12.4 & 10.6 & 11.5 & 0.45 \\
\hline WISE J233226.49-432510.6 & T9: & - & - & 13.5 & 15.7 & 14.6 & 0.92 \\
\hline WISEPC J234446.25+103415.8 & T9 & - & - & 11.6 & 16.6 & 14.1 & 0.83 \\
\hline \multicolumn{8}{|c|}{ Y0-0.5 Dwarfs (distance limit $\left.=10 \mathrm{pc},\left\langle\frac{V}{\nabla_{\max }}\right\rangle=0.30\right)$} \\
\hline WISE J014656.66+423410.0 & Y0 & - & _- & 3.2 & 9.4 & 6.3 & 0.25 \\
\hline WISE J035934.06-540154.6 & Yo & - & - & 16.0 & 11.0 & 13.5 & - \\
\hline WISEPA J041022.71+150248.5 & Y0 & $0.164 \pm 0.024$ & 14 & 3.8 & 6.2 & 6.1 & 0.23 \\
\hline WISE J071322.55-291751.9 & Y0 & - & - & $>4.2$ & 7.1 & 7.1 & 0.36 \\
\hline WISE J073444.02-715744.0 & Yo & - & - & - & 10.7 & 10.7 & - \\
\hline WISEPC J140518.40+553421.4 & Yop? & $0.207 \pm 0.039$ & 14 & $>7.3$ & 6.0 & 4.8 & 0.11 \\
\hline WISEPA J154151.66-225025.2 & Y0.5 & - & - & $>3.0$ & 4.2 & 4.2 & 0.07 \\
\hline WISEPA J173835.53+273258.9 & Yo & $0.111 \pm 0.036$ & 14 & 7.0 & 7.4 & 9.0 & 0.73 \\
\hline WISEPC J205628.90+145953.3 & Yo & - & - & 4.9 & 5.5 & 5.2 & 0.14 \\
\hline WISE J222055.32-362817.5 & Y0 & - & - & 8.4 & 7.8 & 8.1 & 0.53 \\
\hline \multicolumn{8}{|c|}{ Y1-1.5 Dwarfs (distance limit $\left.=10 \mathrm{pc},\left\langle\frac{V}{V_{\max }}\right\rangle=0.14\right)$} \\
\hline WISE J035000.32-565830.2 & Y1 & $0.238 \pm 0.038$ & 14 & 2.3 & 3.1 & 4.2 & 0.07 \\
\hline WISE J053516.80-750024.9 & $\geq \mathrm{Y} 1:$ & $0.170 \pm 0.044$ & 14 & 2.4 & 3.6 & 5.9 & 0.21 \\
\hline \multicolumn{8}{|c|}{ Y2-2.5 Dwarfs (distance limit $\left.=10 \mathrm{pc},\left\langle\frac{V}{V \max }\right\rangle=0.55\right)$} \\
\hline WD $0806-661 \mathrm{~B}$ & $(\mathrm{Y} ?)$ & $0.0522 \pm 0.0017$ & 16 & - & - & 19.2 & - \\
\hline WISEPA J182831.08+265037.8 & $\geq \mathrm{Y} 2$ & $0.122 \pm 0.013$ & 13 & - & - & 8.2 & 0.55 \\
\hline
\end{tabular}

Note. - Parallax references: (1) Marocco et al. (2010), (2) Lucas et al. (2010), (3) Artigau et al. (2010), (4) Perryman et al. (1997), (5) Vrba et al. (2004), (6) van Leeuwen (2007), (7) Schilbach et al. (2009), (8) Dahn et al. (2002), (9) Tinney et al. (2003), (10) Henry et al. (2006), (11) Dupuy \& Liu (2012), (12) Kirkpatrick et al. (2011), (13) Beichman et al. (in prep.), (14) Marsh et al. (in prep.), (15) Burgasser et al. (2008), (16) Subasavage et al. (2009) (17) van Altena et al. (1995), (18) Karatas et al. (2004).

${ }^{\mathrm{a}}$ This object may not exist.

${ }^{\mathrm{b}}$ Distance estimate from Cuby et al. (1999). 
'Geißler et al. (2011) estimates that the primary (if this is a true binary) has a type of T2. The $J$-band combined light of the system is $16.05 \mathrm{mag}$ from Chiu et al. (2006). The $J$-band absolute magnitude relation from Looper et al. (2008) gives $M_{J}=14.45 \mathrm{mag}$ for a T2 dwarf, meaning that this system is no closer than 20.9 pc.

${ }^{\mathrm{d}}$ Distance estimate from Lodieu et al. (2009).

${ }^{\mathrm{e}}$ Distance estimate from Stumpf et al. (2010).

${ }^{\mathrm{f}}$ Distance estimate from Burgasser et al. (2012).

${ }^{\mathrm{g}}$ Assumed distance is that derived from the W2 estimate only because the $H$-band measurement is very uncertain.

${ }^{\mathrm{h}}$ Assumed distance is that derived from the $H$ estimate only because the W2 measurement is blended with a nearby source.

${ }^{\mathrm{i}}$ Distance estimate from Masters et al. (2012). 
Table 9. $\quad$ Updated Space Densities for the All-sky, Volume-limited Sample of Late-T and Y Dwarfs

\begin{tabular}{|c|c|c|c|c|c|c|}
\hline $\begin{array}{l}\text { Spectral Type } \\
\text { Range } \\
\text { (1) }\end{array}$ & $\begin{array}{c}\text { Approx. } T_{e f f} \\
\text { Range }(\mathrm{K}) \\
(2)\end{array}$ & $\begin{array}{c}d_{\max } \\
(\mathrm{pc}) \\
(3)\end{array}$ & $\begin{array}{l}\text { Total \# } \\
\text { objects } \\
(4)\end{array}$ & $\begin{array}{l}\text { Obs. Space Density } \\
\left(\# / p c^{3}\right) \\
(5)\end{array}$ & $\begin{array}{c}<V / V_{\max }> \\
(6)\end{array}$ & $\begin{array}{l}\text { \# per } 100 \mathrm{~K} \text { Bin } \\
\text { w/in } 10 \mathrm{pc} \\
(7)\end{array}$ \\
\hline Т6-Т6.5 & $900-1050$ & 20 & 38 & $1.1 \mathrm{e}-3$ & 0.41 & 3.1 \\
\hline T7-T7.5 & $750-900$ & 20 & 31 & $9.3 e-4$ & 0.37 & 2.6 \\
\hline T8-T8.5 & $600-750$ & 20 & 46 & $1.4 \mathrm{e}-3$ & 0.35 & 3.9 \\
\hline Т9-Т9.5 & $450-600$ & 15 & $22^{\mathrm{a}}$ & $1.6 \mathrm{e}-3^{\mathrm{a}}$ & 0.44 & $4.5^{\mathrm{a}}$ \\
\hline Y0-Y0.5 & $300-450$ & 10 & $8^{a}$ & $1.9 \mathrm{e}-3^{\mathrm{a}}$ & 0.30 & $5.3^{\mathrm{a}}$ \\
\hline$\geq \mathrm{Y} 1$ & $150-300$ & 10 & $3^{\mathrm{a}}$ & $7.2 \mathrm{e}-4^{\mathrm{a}}$ & 0.28 & $2.0^{\mathrm{a}}$ \\
\hline
\end{tabular}

${ }^{a}$ These values should be taken as lower limits only, given that follow-up of $\geq \mathrm{T} 9$ dwarf discoveries within this volume is still incomplete. 


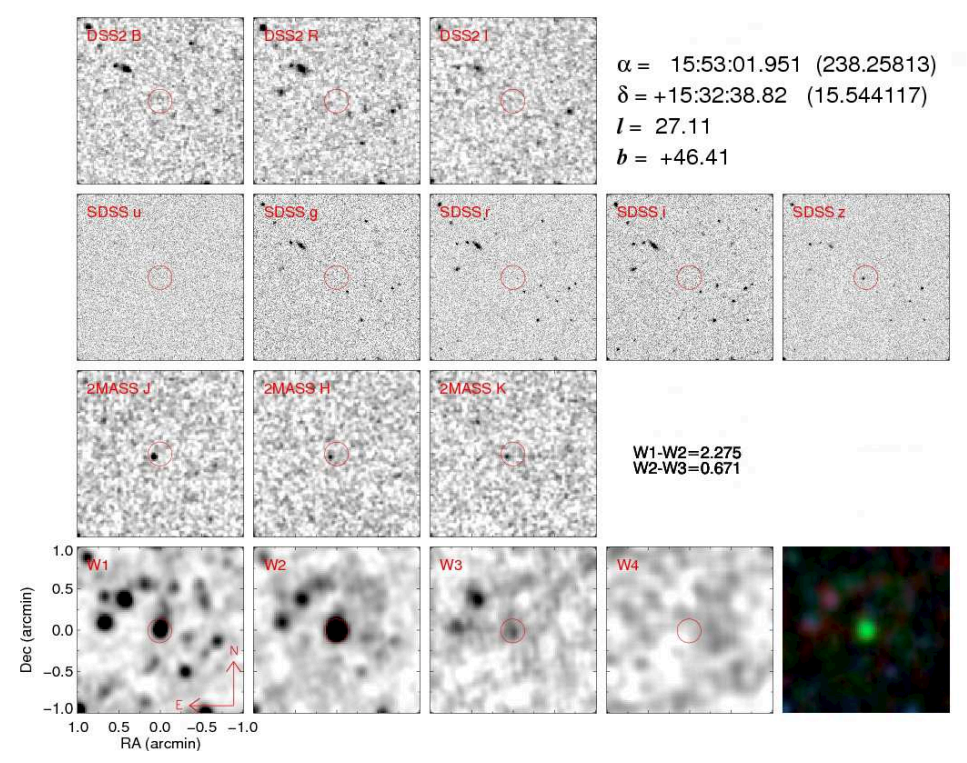

Fig. 1. - Images of one of our brown dwarf candidates, the previously known T7 dwarf 2MASSI J1553022+153236 from Burgasser et al. (2002). Each row in this image represents a different survey and each image shows a $2 \times 2$ arcminute region at a different wavelength. The top row shows $B, R$, and $I$ images from the Digitized Sky Survey 2 (DSS2). The second row shows $u, g, r, i$, and $z$ images from the Sloan Digital Sky Survey (SDSS; York et al. 2000). The third row shows $J, H$, and $K_{s}$ images from the Two-Micron All-Sky Survey (2MASS; Skrutskie et al. 2006). The last row shows the W1, W2, W3, and W4 images from WISE along with a three-color image comprised of W1 (blue), W2 (green), and W3 (red). Sexagesimal and decimal RA $(\alpha)$ and Dec $(\delta)$ coordinates of the source are given in the upper right, along with the Galactic longitude $(l)$ and latitude $(b)$ in decimal degrees. Also shown are the W1-W2 and W2-W3 colors from WISE. A red circle is shown at the location of the candidate identified in WISE. For this object, the source is detected only at SDSS $z$, all three 2MASS bands, and the three shortest wavelength bands of WISE; detections at these wavelengths are common for nearby, bright T dwarfs. Motion is seen from the earliest epochs (2MASS and SDSS) to the latest (WISE), further confirming this as a good candidate. 


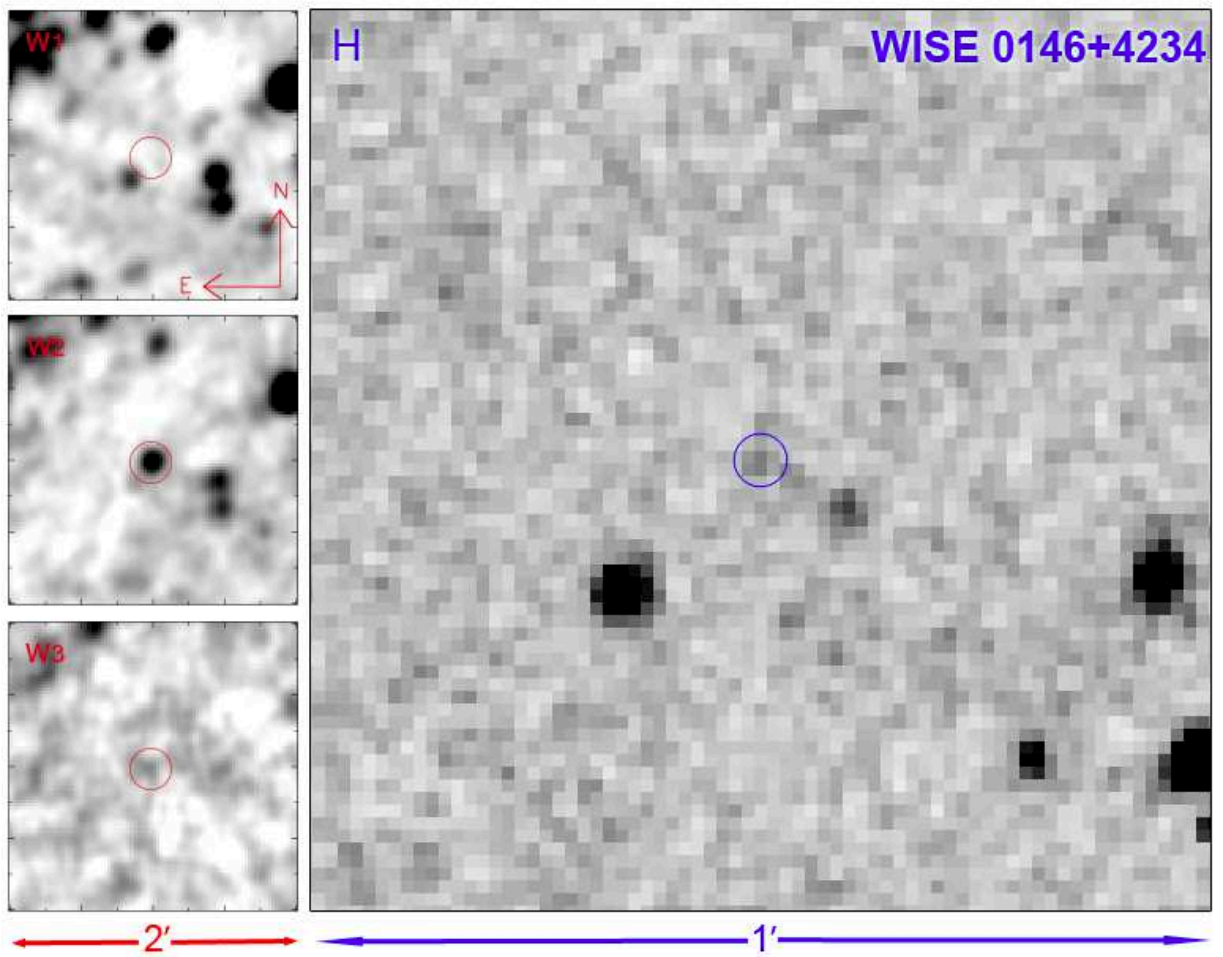

Fig. 2.- Finder charts for all thirteen known WISE Y dwarf discoveries. Along the left are $2 \times 2$ arcminute images from WISE in bands W1, W2, and W3 with the location of the $\mathrm{Y}$ dwarf shown by the red circle. The large image on the right is a $1 \times 1$ arcminute zoom taken in the the near-infrared; the $\mathrm{Y}$ dwarf is circled in blue. In all images, north is up and east is to the left. Note that the field for WISE $0535-7500$ is located toward the outskirts of the Large Magellanic Cloud, which is the reason for the high source density. For WISE 0713-2917, our near-infrared image on the right does not completely fill the $1 \times 1$ arcminute field allotted for it. For WISE 1541-2250, the prominent white ring on the large image is a latent artifact caused by a bright star during the dithering process. 


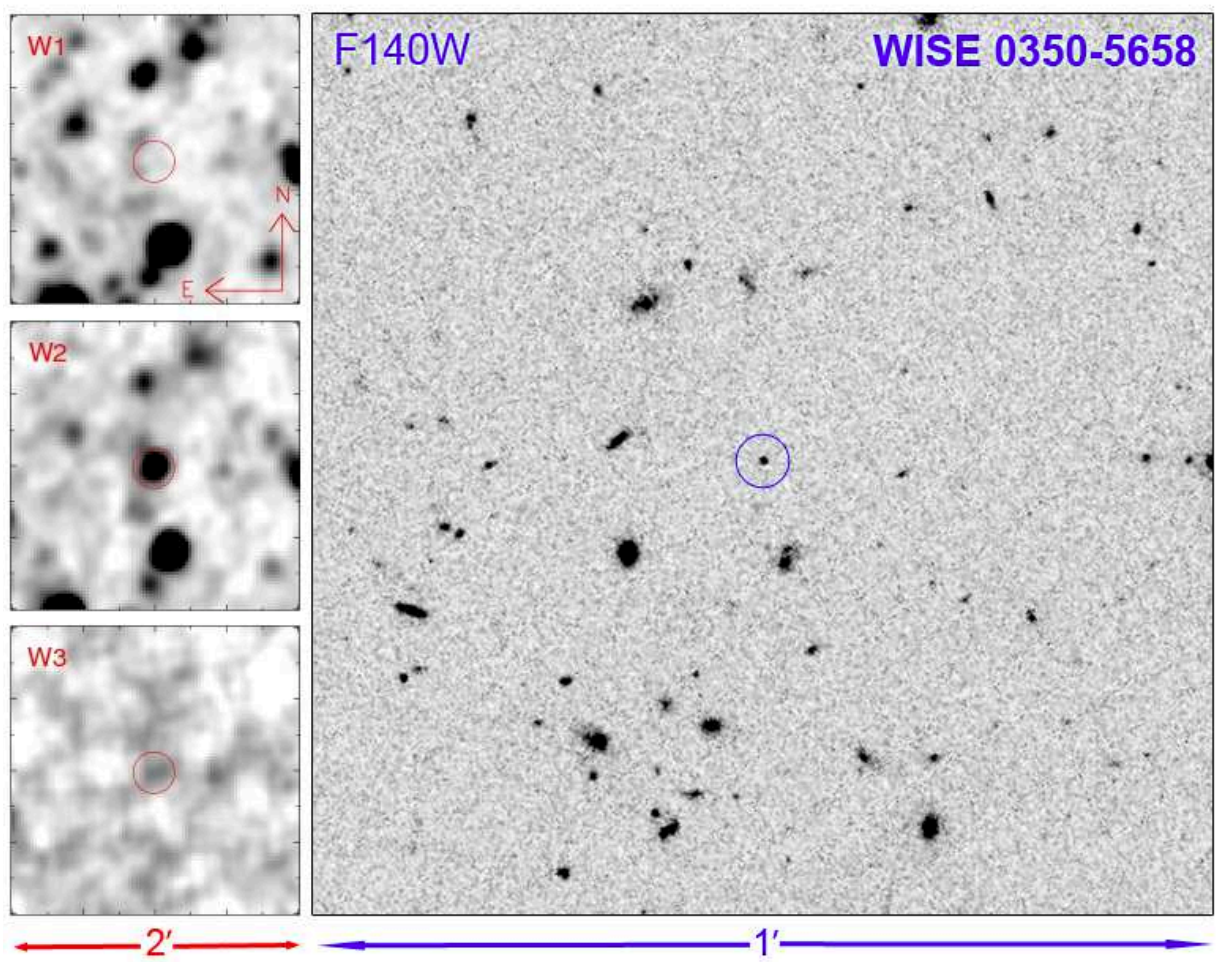

Fig. 2.- Continued. 

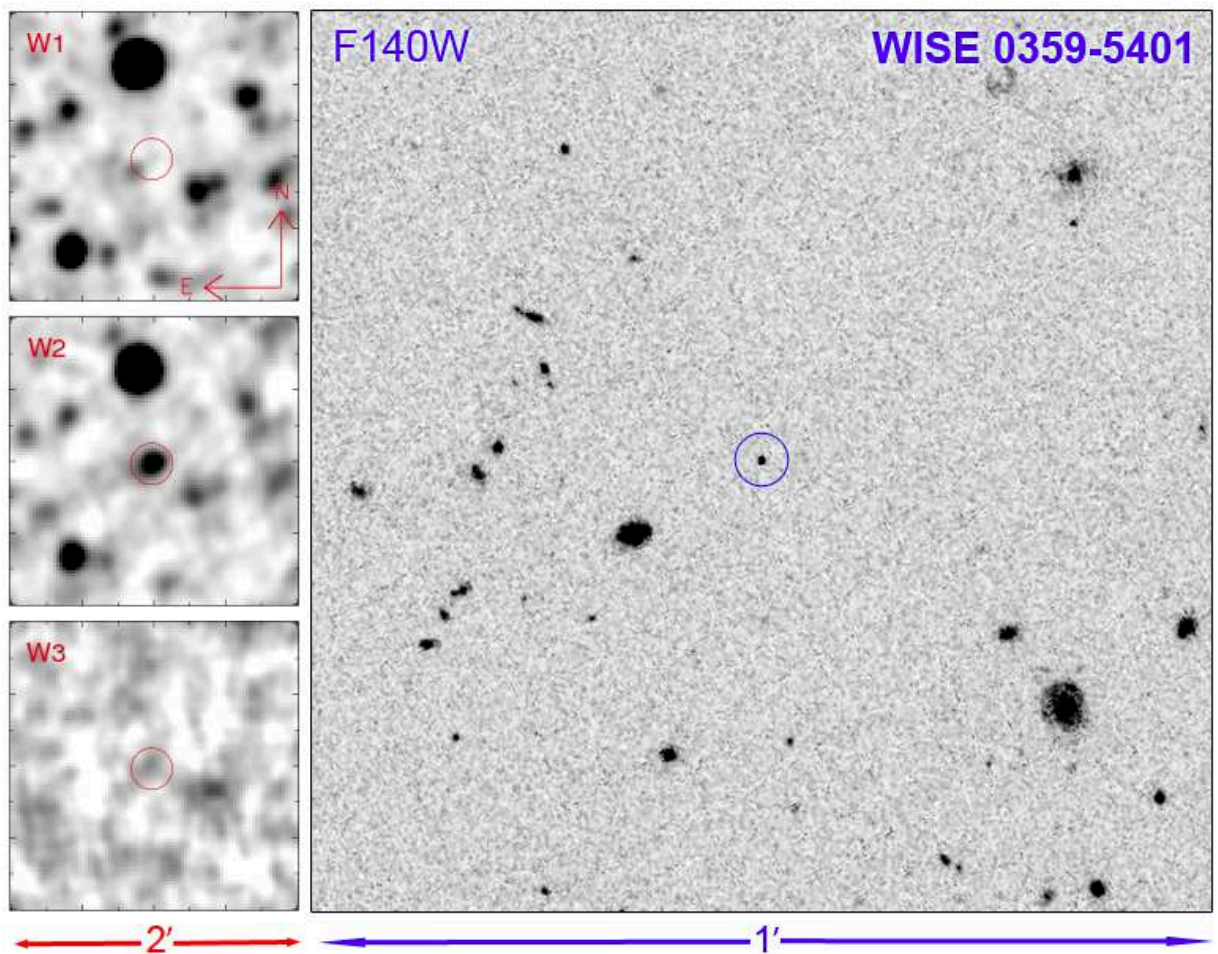

Fig. 2.- Continued. 


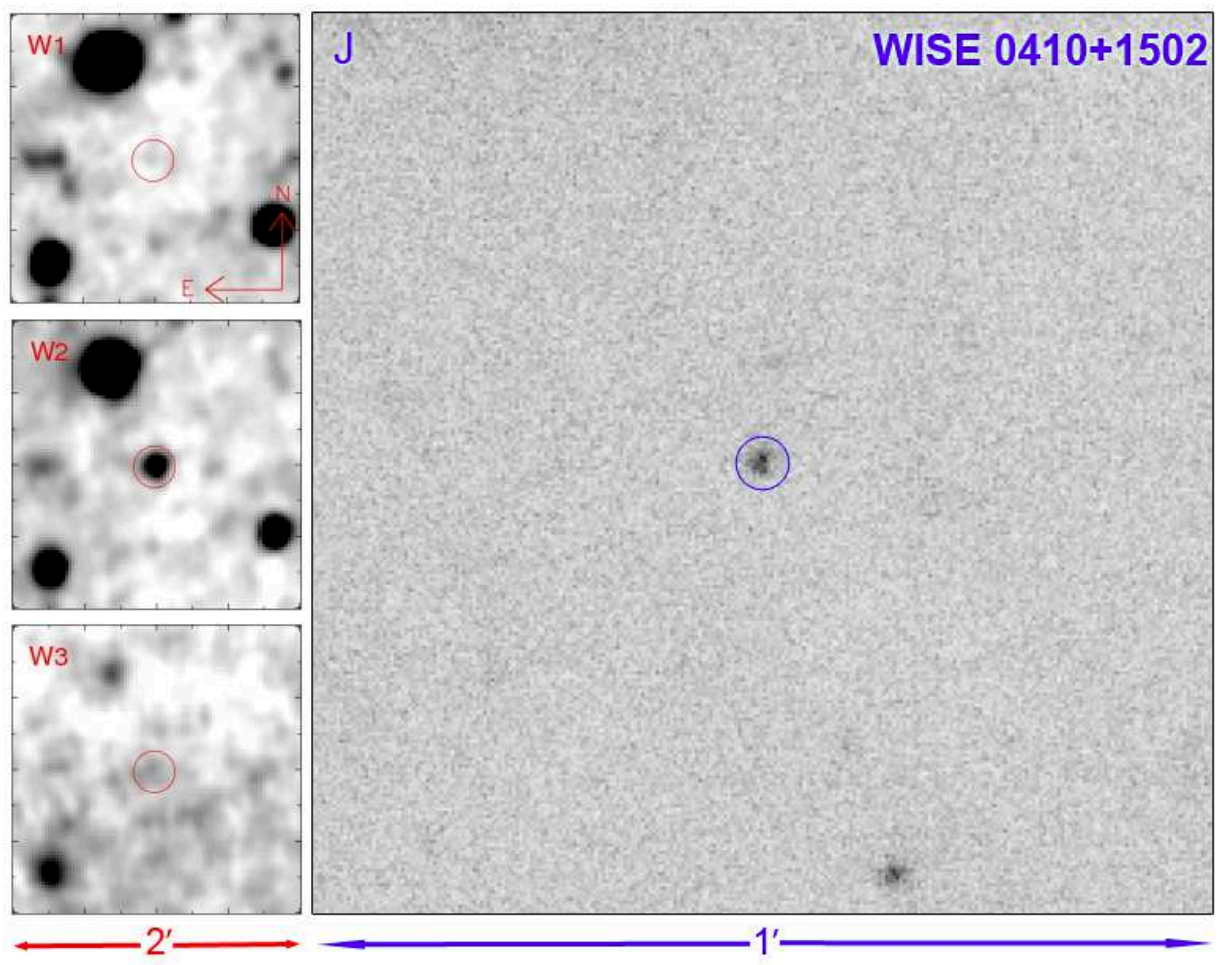

Fig. 2.- Continued. 


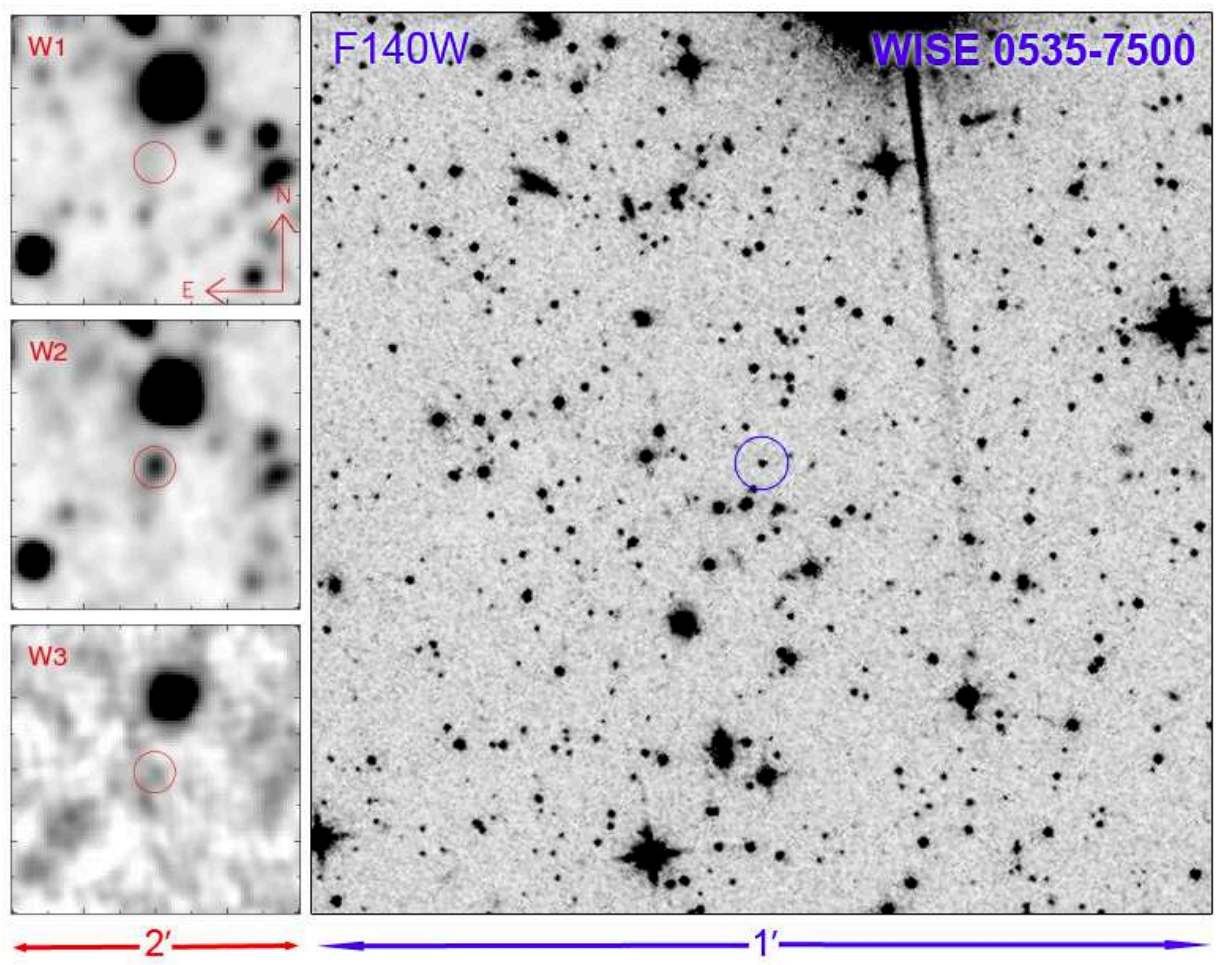

Fig. 2.- Continued. 


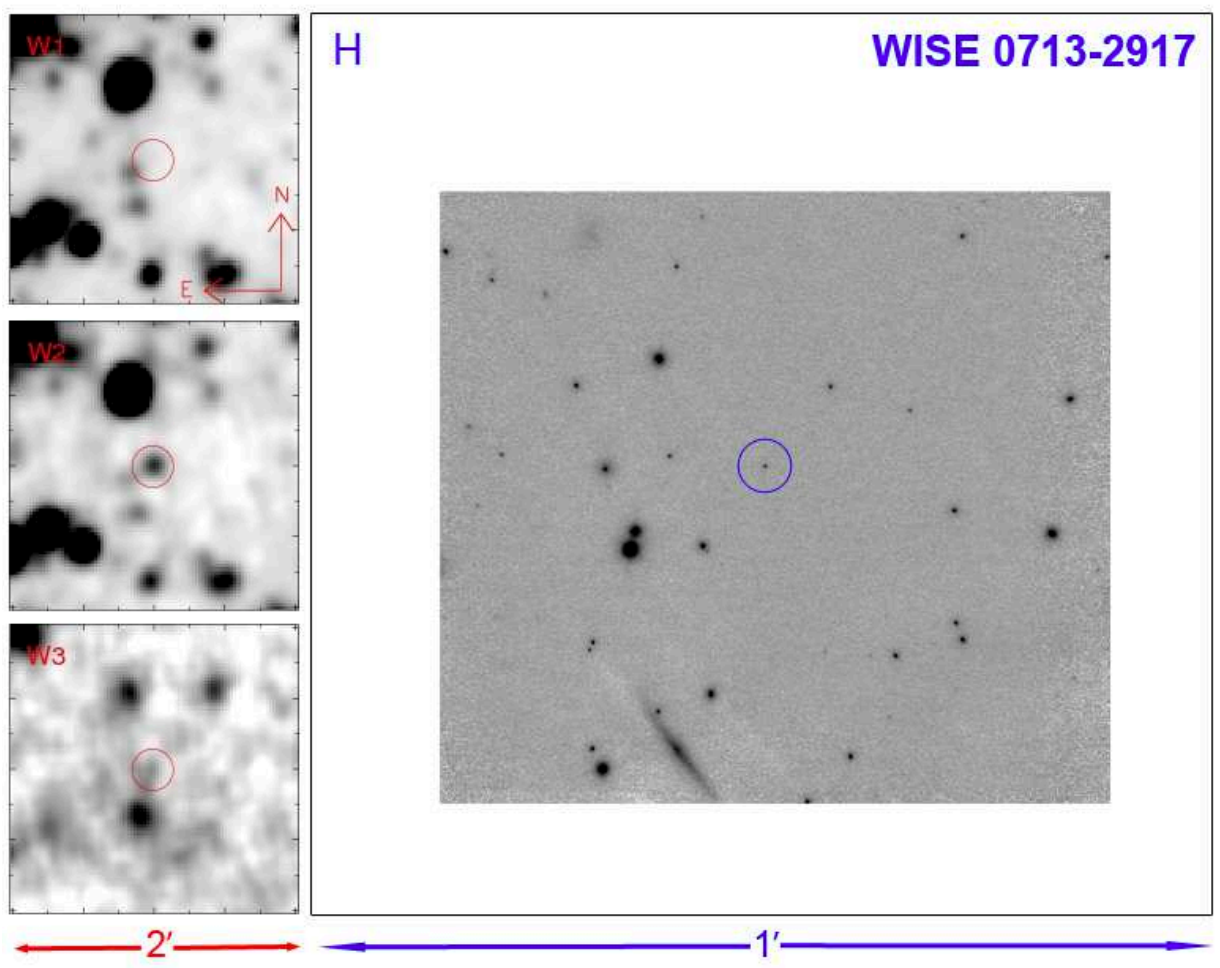

Fig. 2.- Continued. 


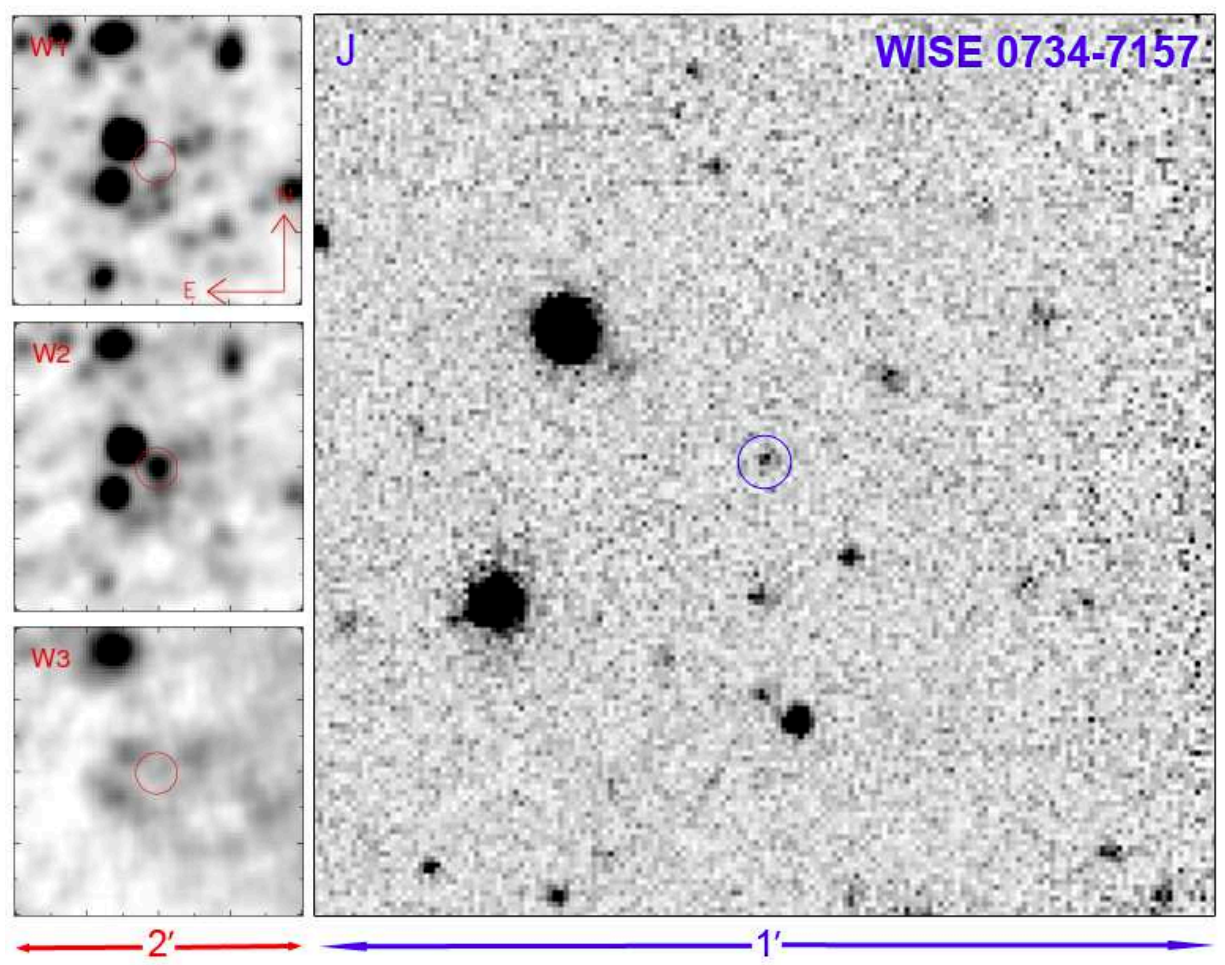

Fig. 2.- Continued. 


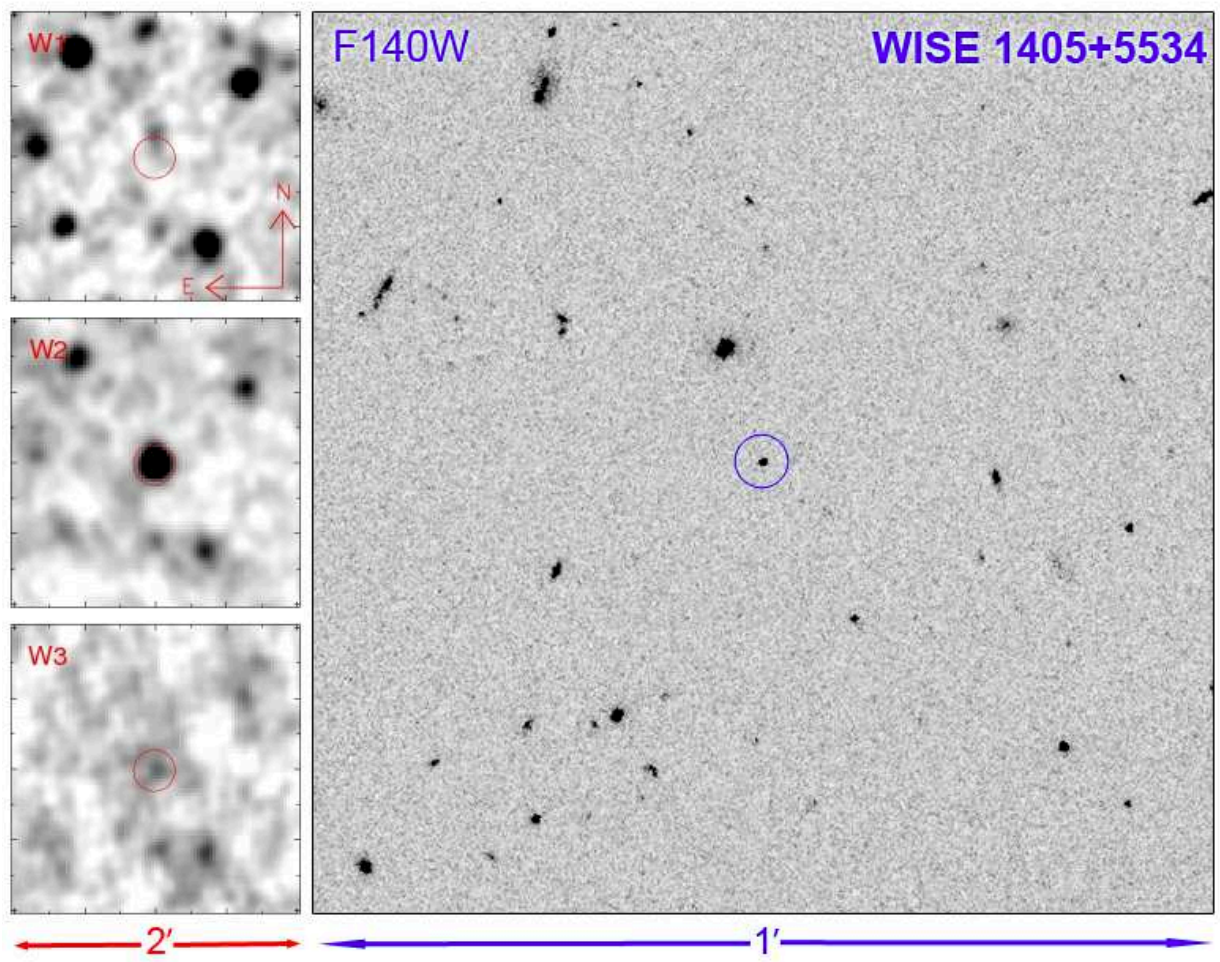

Fig. 2.- Continued. 


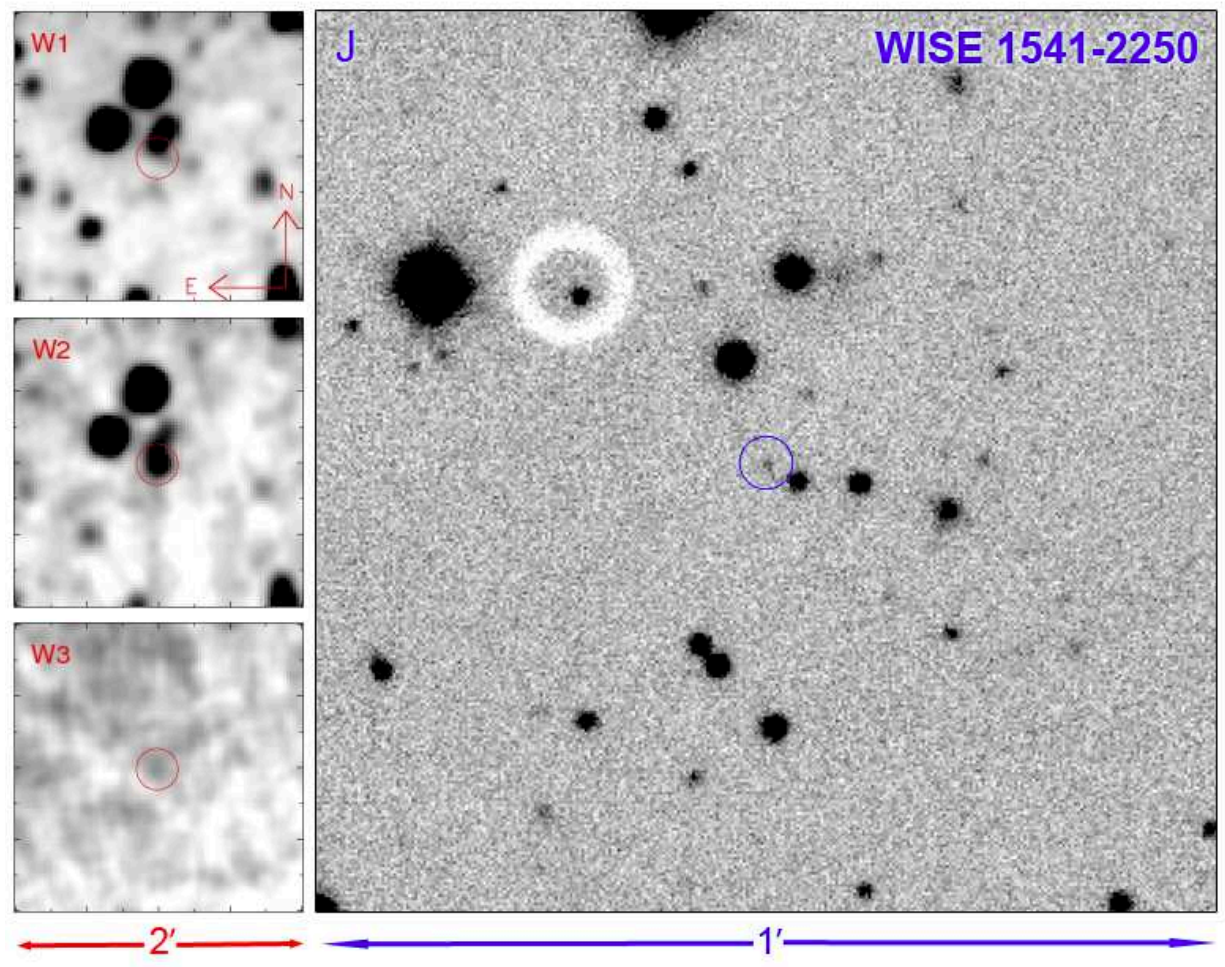

Fig. 2.- Continued. 


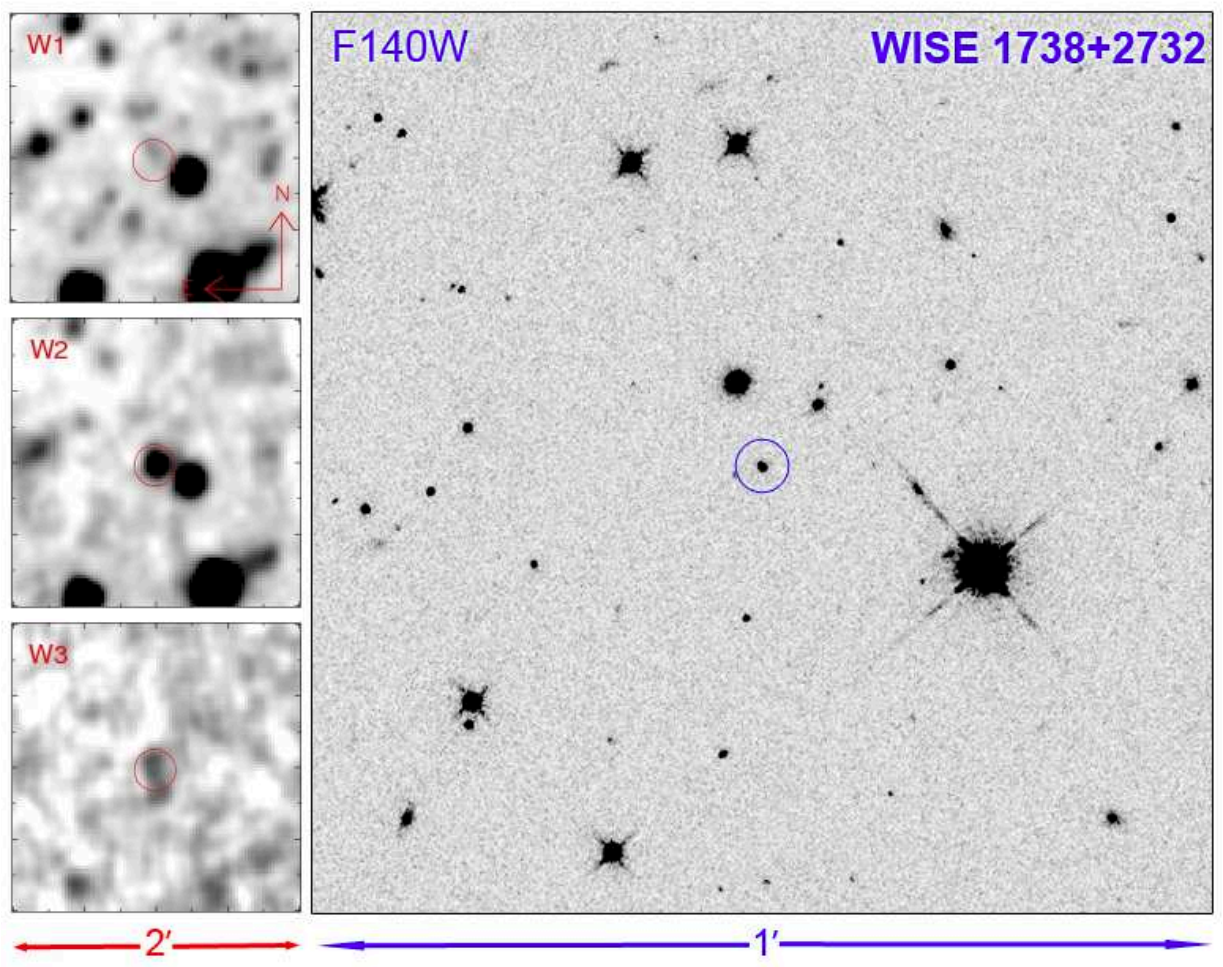

Fig. 2.- Continued. 


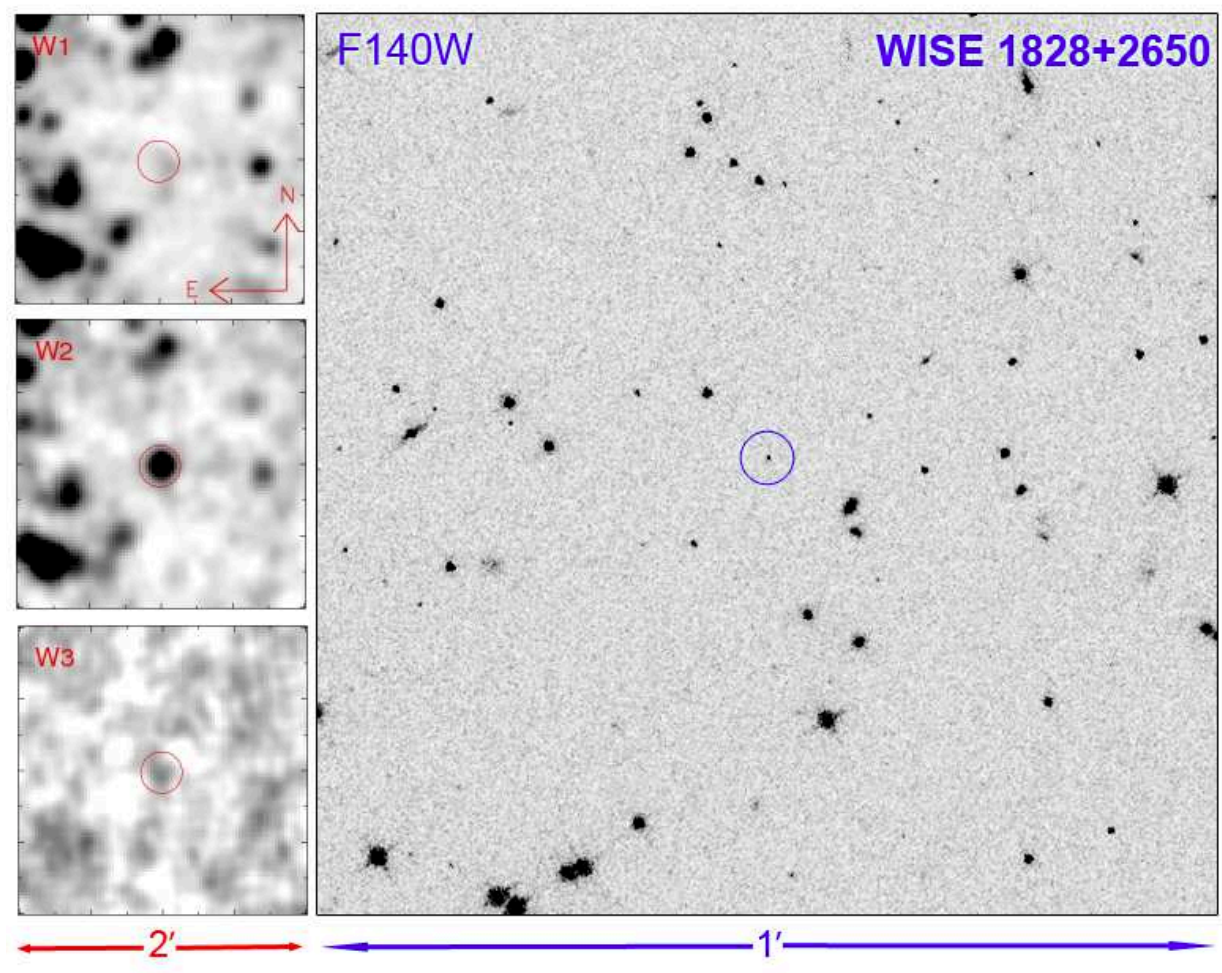

Fig. 2.- Continued. 


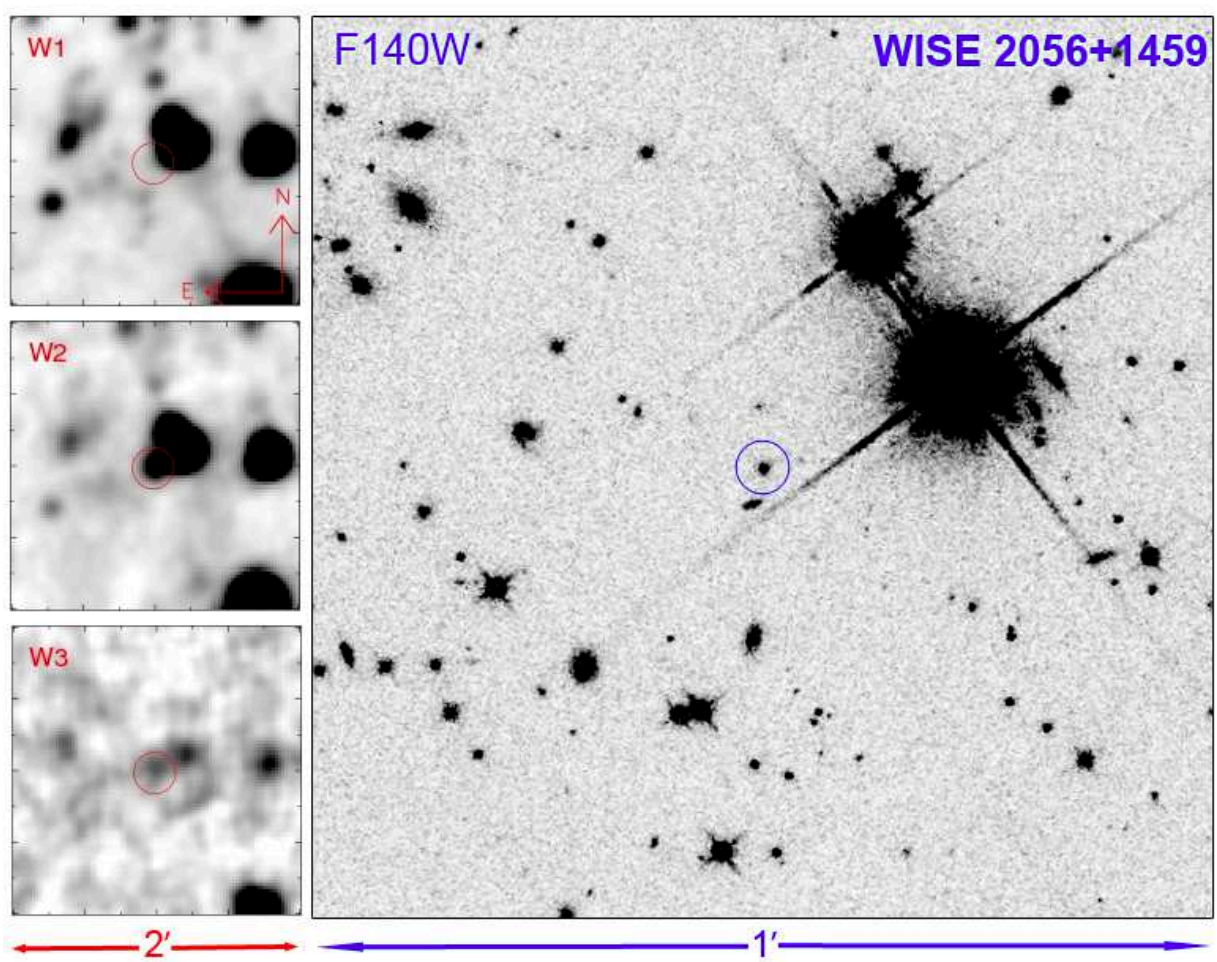

Fig. 2.- Continued. 


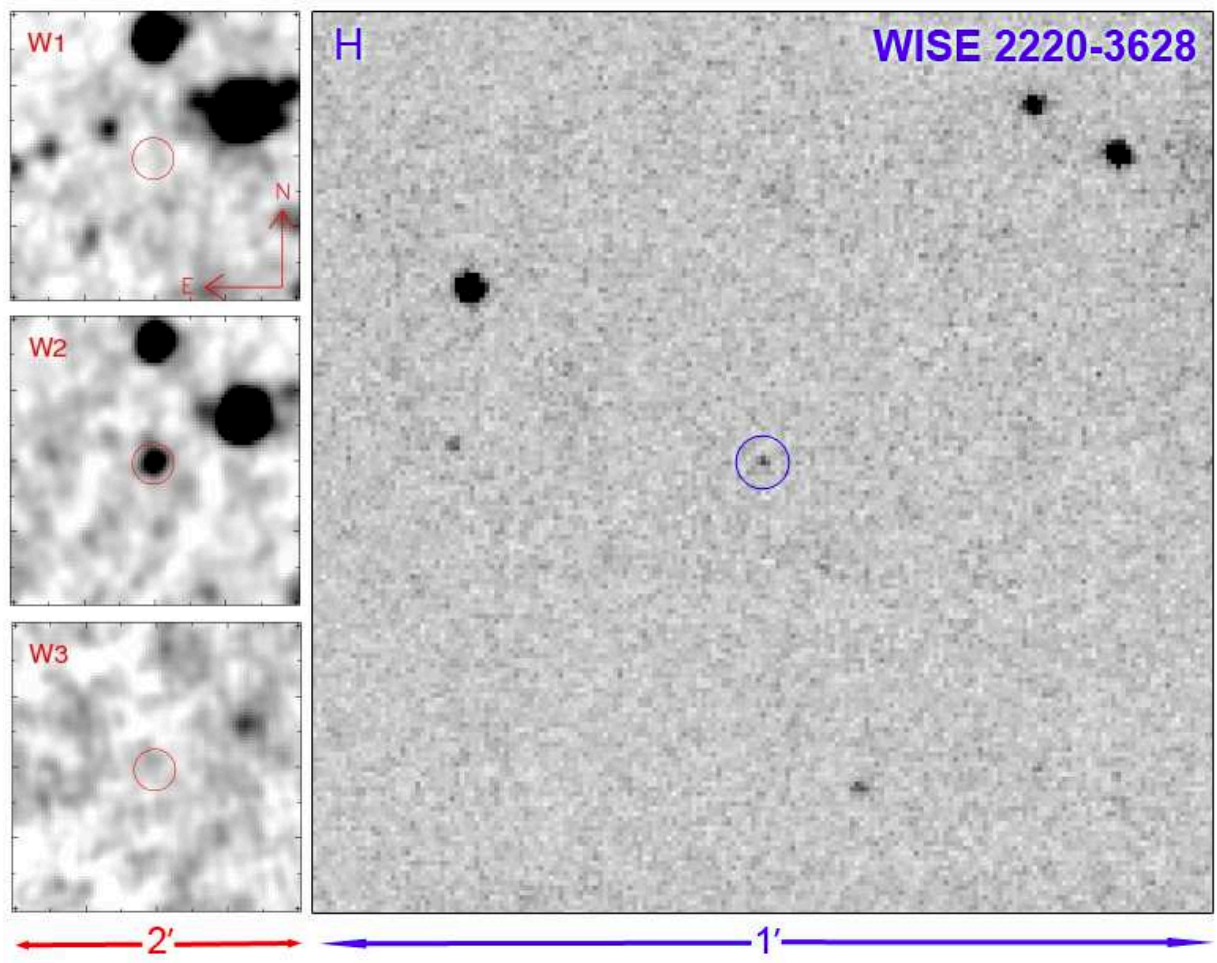

Fig. 2.- Continued. 


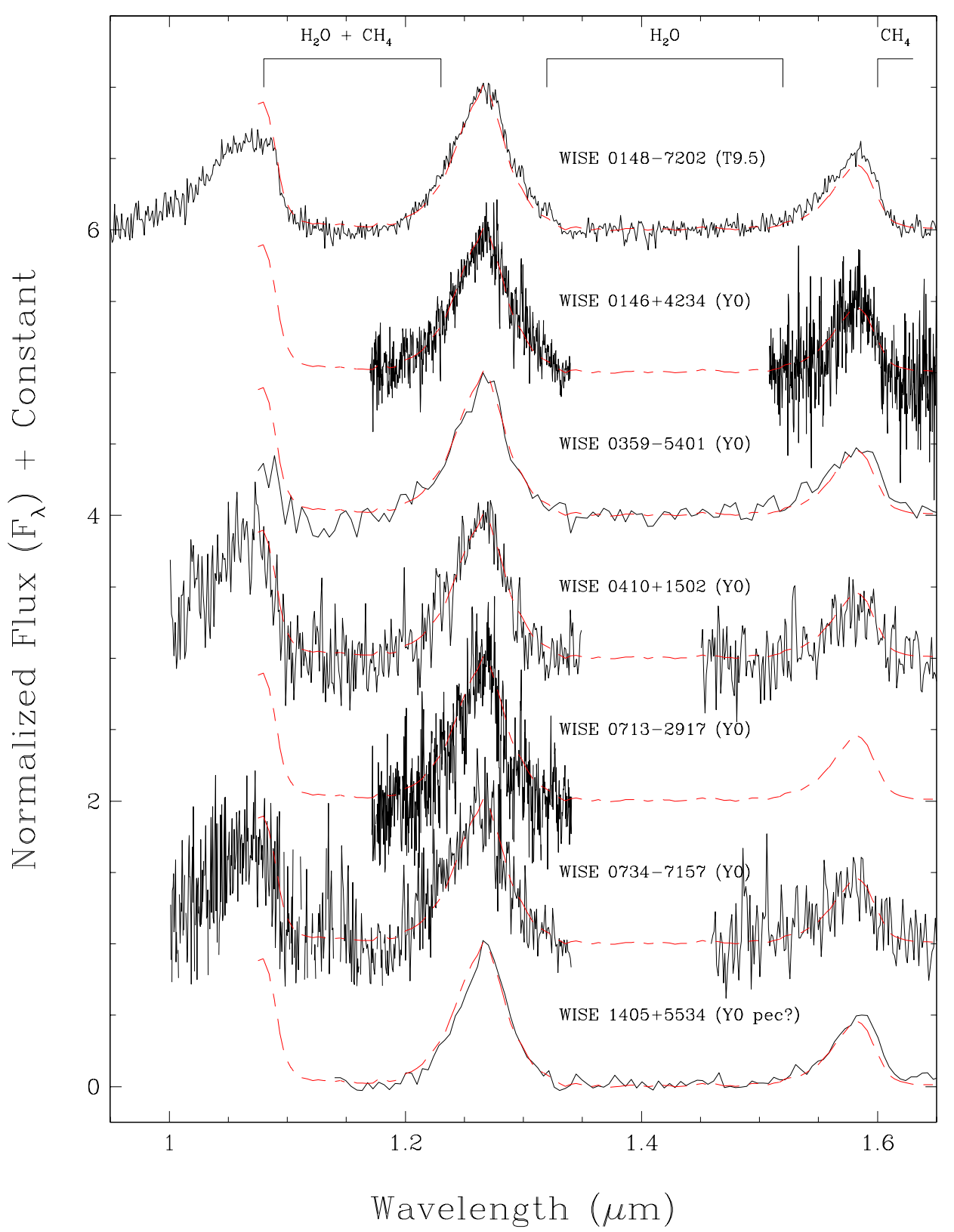

Fig. 3.- Along with Fig 4 and Fig 5 , the spectra of all known Y dwarfs together with a comparison spectrum of the T9.5 dwarf WISE 1048-7202. Each spectrum is normalized to one at its peak in the $J$-band and integral offsets have been added to separate the spectra vertically. Overplotted on each spectrum is the Y0 dwarf spectral standard WISE 1738+2732 (dashed red curve). For NIRSPEC data taken in the N5 configuration ( $H$-band), the normalization has been set so that the $H$-band peak of the $\mathrm{Y}$ dwarf matches the $H$-band peak of the spectral standard. 


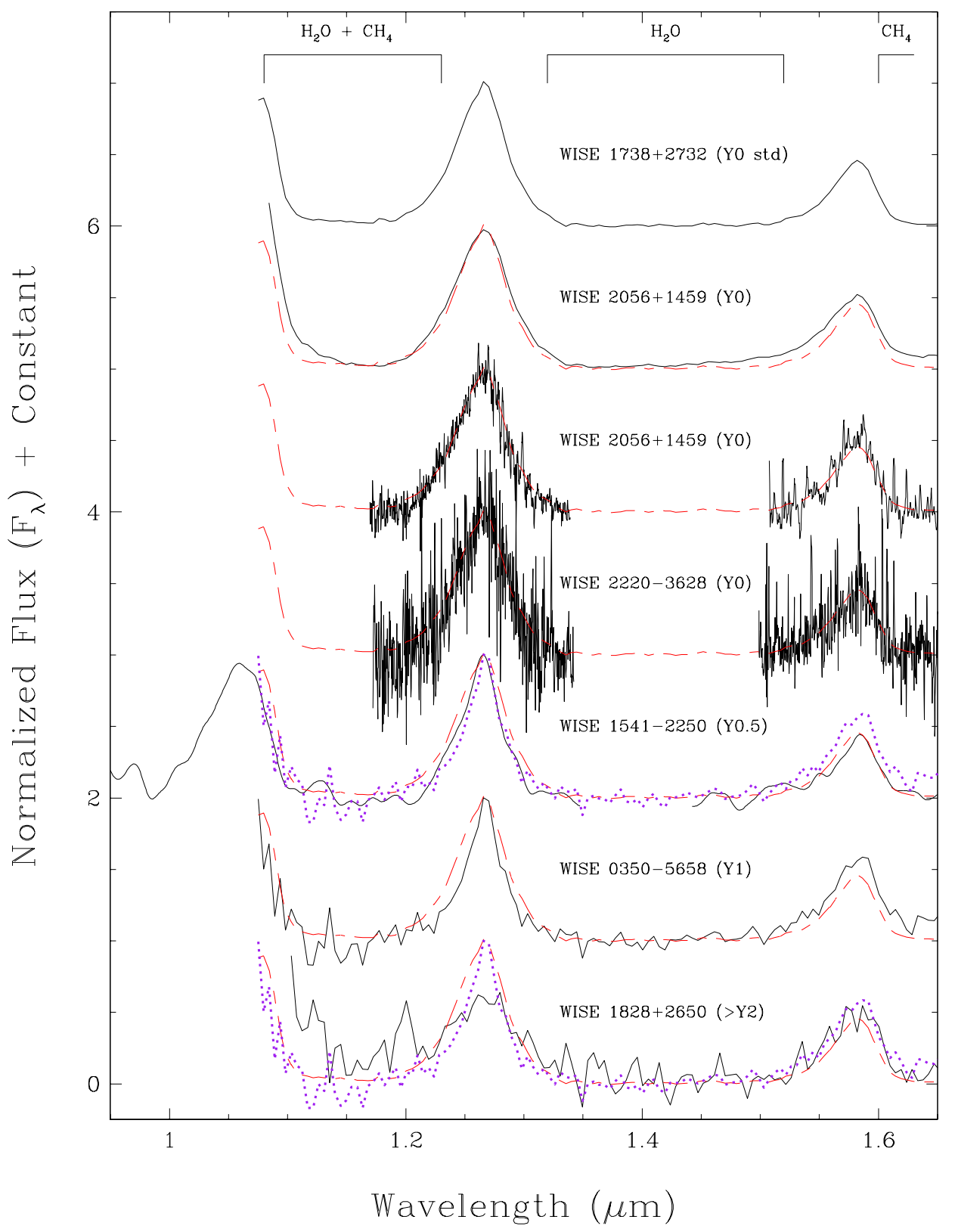

Fig. 4.- Spectra of Y dwarfs (continued). The spectrum of the Y1 standard, WISE 03505658 , is shown by the purple dotted line and is normalized to one at the $J$-band peak. See caption to Fig 3 for other details. 


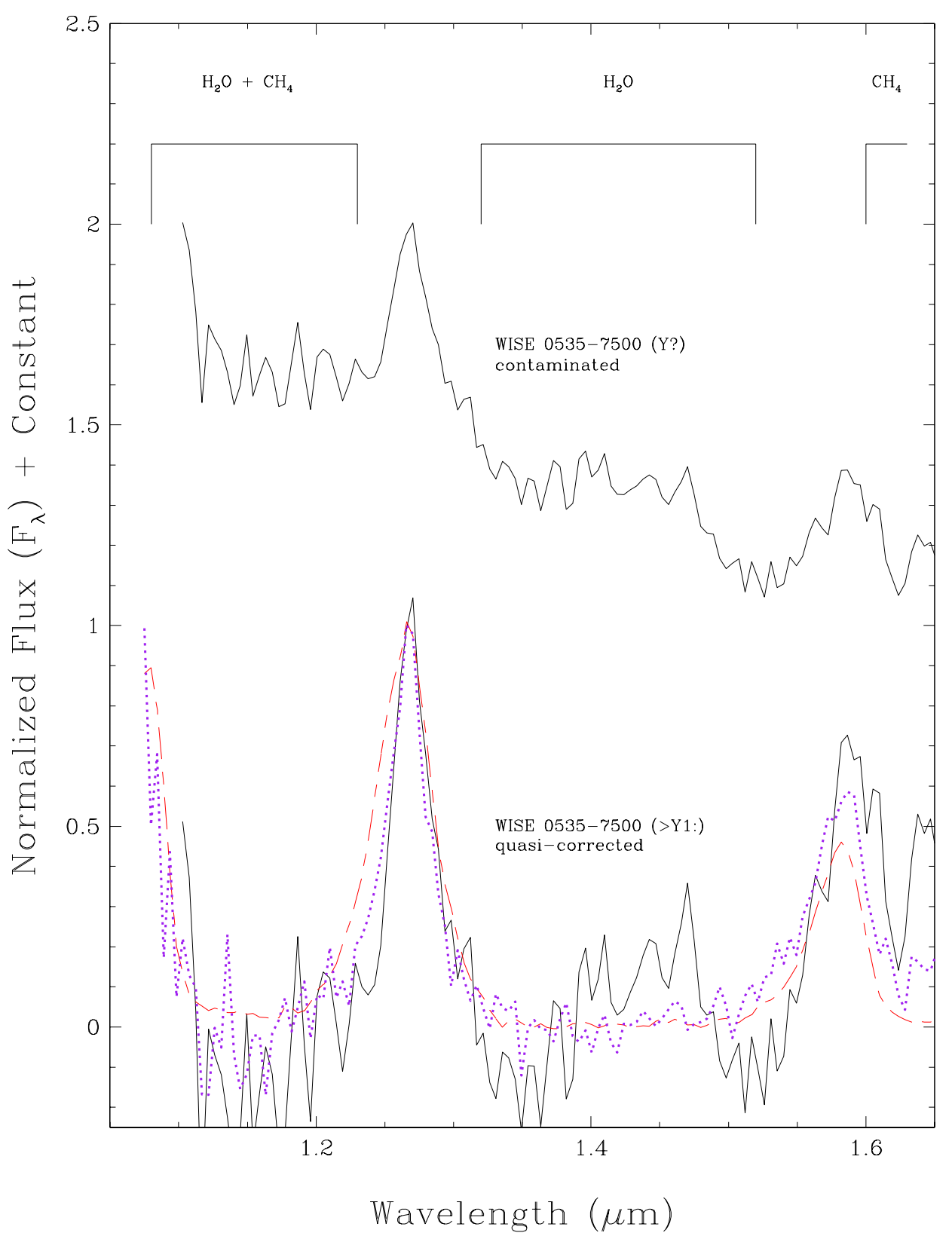

Fig. 5. - Spectra of Y dwarfs (continued). The spectrum of WISE 0535-7500 is shown in black both before (above) and after (below) subtraction of the overlapping field spectrum. Both spectra are normalized to one at the peak of $J$-band, and the top spectrum is offset by one in the vertical direction to separate it from the lower spectrum. See caption to Fig 3 and Fig 4 for other details, and see the text for a description of the correction applied. 


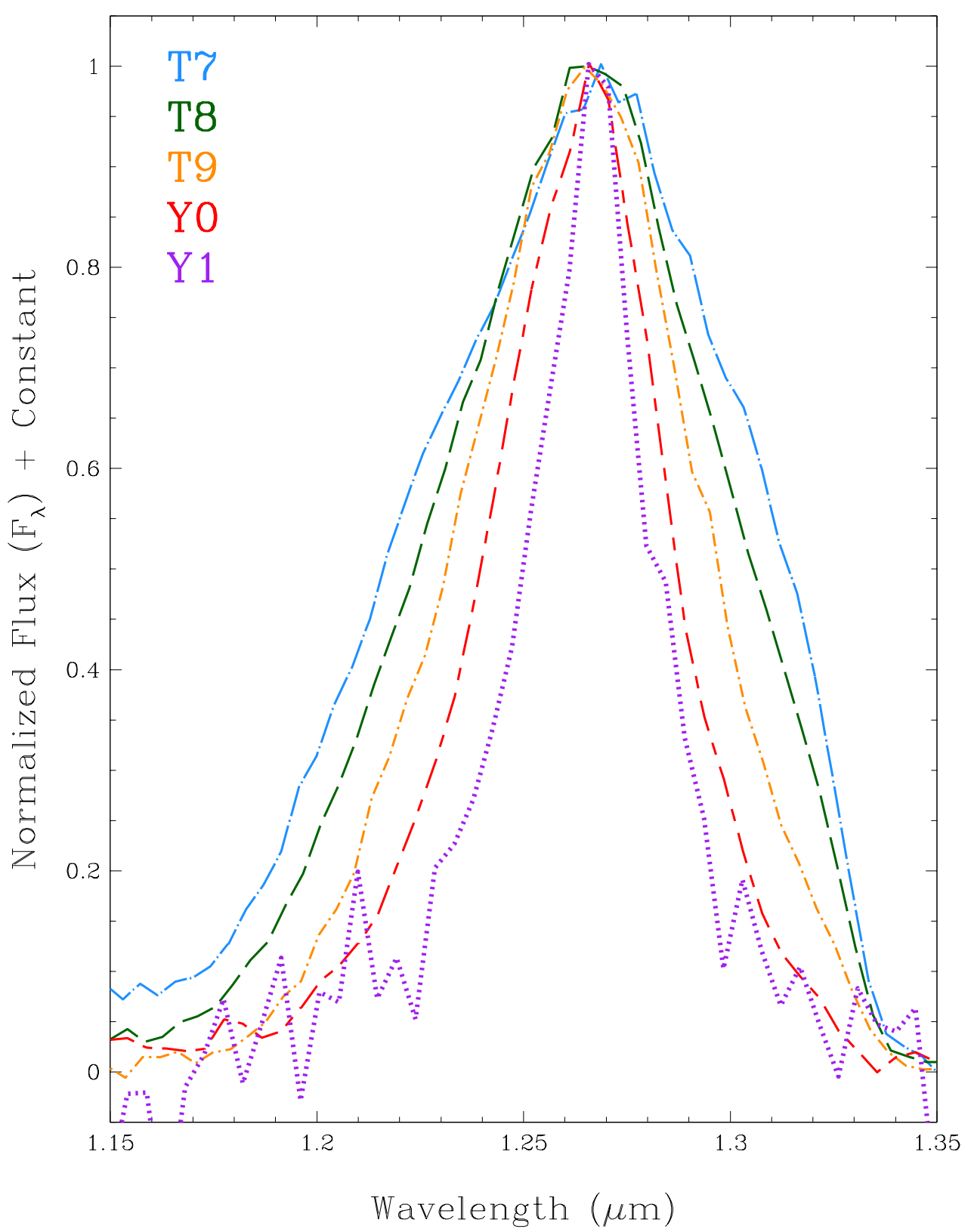

Fig. 6.- Overplots of the $J$-band spectra of the T7, T8, T9, and Y0 standards 2MASS 0727+1710 (light blue, dot/long-dash; Burgasser et al. 2002), 2MASS 0415-0935 (green, dash; Burgasser et al. 2002), UGPS 0722-0540 (gold, dot/short-dash; Lucas et al. 2010), and WISE 1732+2732 (red, long-dash/short-dash; Cushing et al. 2011), respectively, along with our proposed Y1 standard, WISE 0350-5658 (purple, dot). All spectra are normalized to one at their peak flux. 


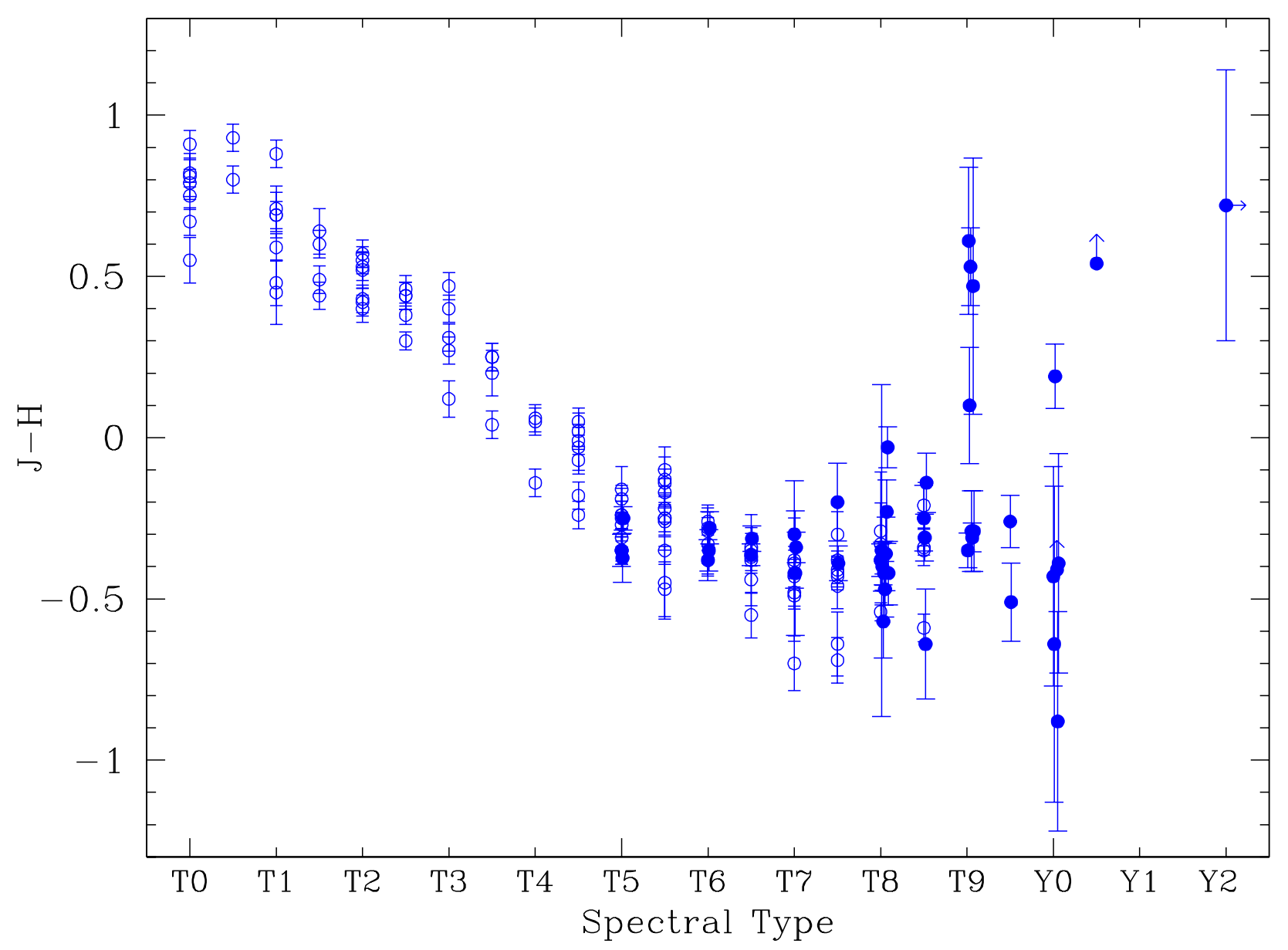

Fig. 7.- A plot of the $J-H$ color (on the MKO-NIR filter system) as a function of spectral type from T0 through Y2. Small offsets have been added to some of the spectral type values to ease visualization of individual data points. Colors for $\mathrm{Y}$ dwarfs from Table 2 and $\mathrm{T}$ dwarfs from Kirkpatrick et al. (2011) and Mace et al. (in prep.) are shown by solid blue points. Colors of other T dwarfs from Leggett et al. (2010) are shown by open blue points. 

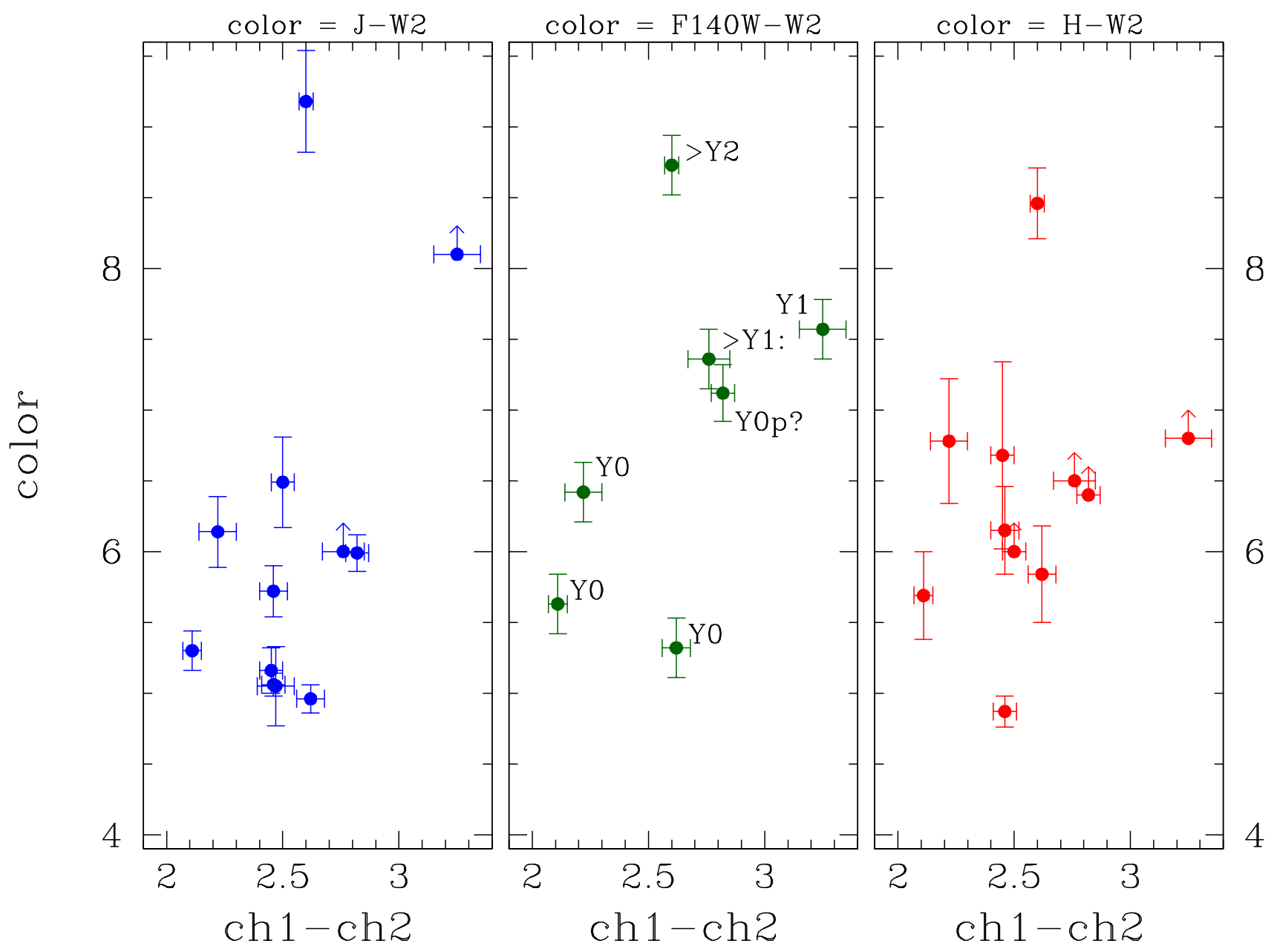

Fig. 8.- Various near-infrared/WISE colors plotted as a function of the Spitzer ch1-ch2 color. From left to right are shown the $J-W 2$ color (blue), F140W-W2 color (green), and $H-\mathrm{W} 2$ color (red). All panels are shown with the same scales on the $x$ and $y$ axes. In the F140W-W2 panel, spectral types are shown next to each of the points. 


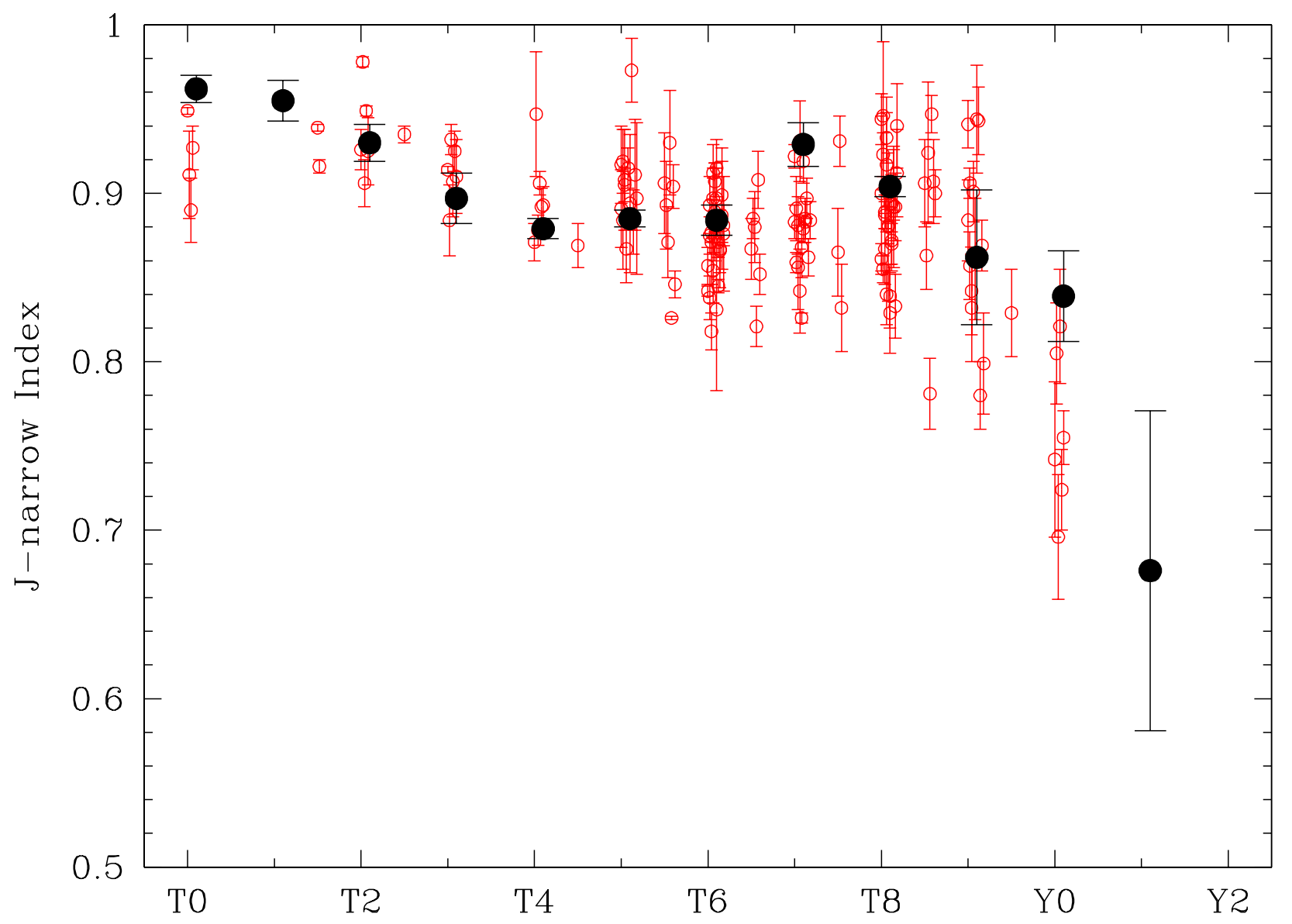

Fig. 9.- Values of our newly defined J-narrow index as a function of spectral type. Objects are taken from this paper, Mace et al. (in prep.), Cushing et al. (2011), and Kirkpatrick et al. (2011). See Mace et al. for details concerning these computations. Spectral standards are shown by solid black points and other objects are shown by open red points. Small toggles have been added to the spectral type values to reduce the amount of overlap in each spectral bin. With the exception of the Y1 standard, only those objects with $J$-narrow errors of less than 0.05 are plotted. 


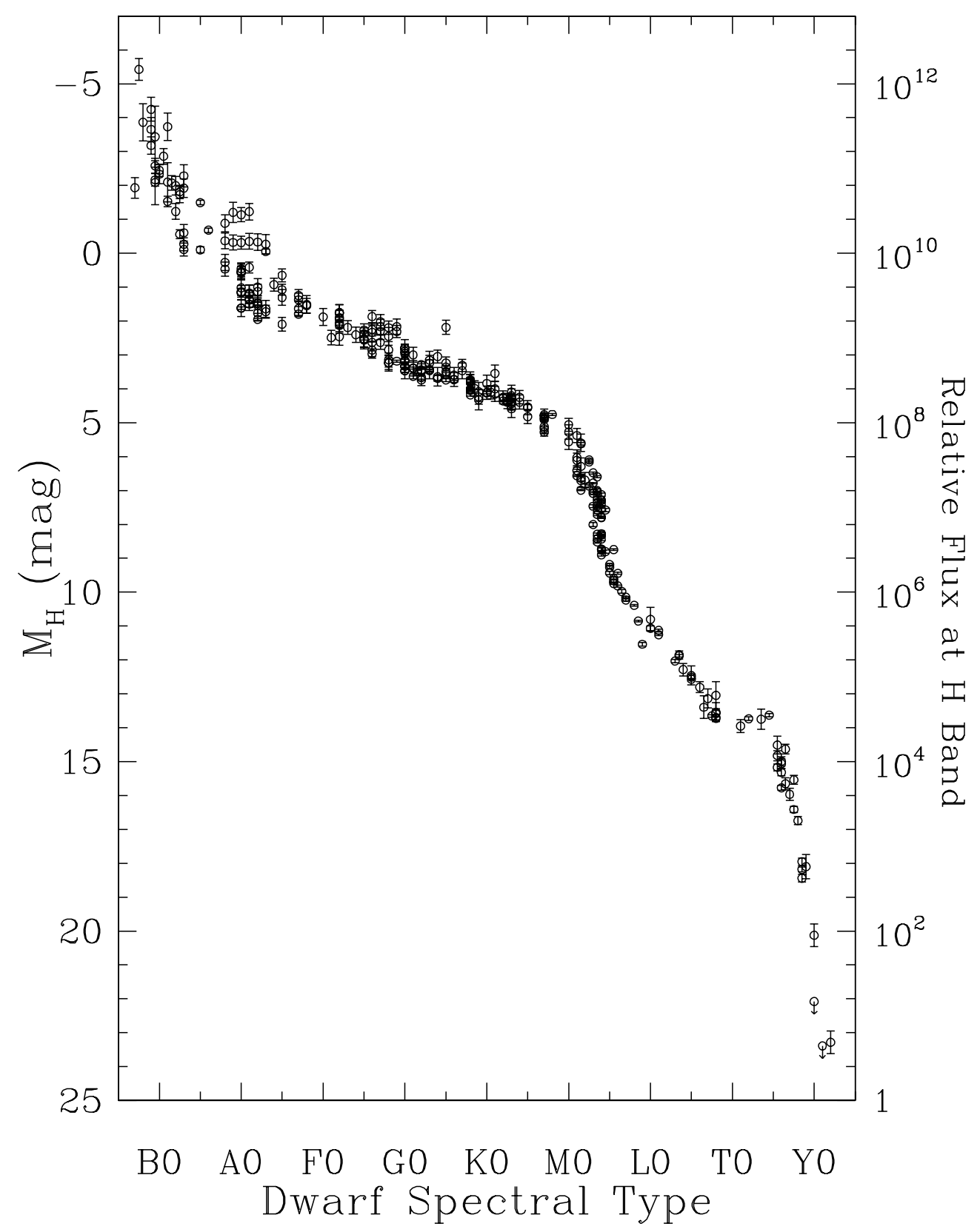

Fig. 10.- A Hertzsprung-Russell diagram at $H$-band showing the $\mathrm{Y}$ dwarfs in context with other field brown dwarfs and main sequence stars. The intrinsically faintest Y dwarf so far recognized is roughly twelve orders of magnitude fainter than an O-type main sequence star at this wavelength. See Section 4.1 for details about the sample plotted. 


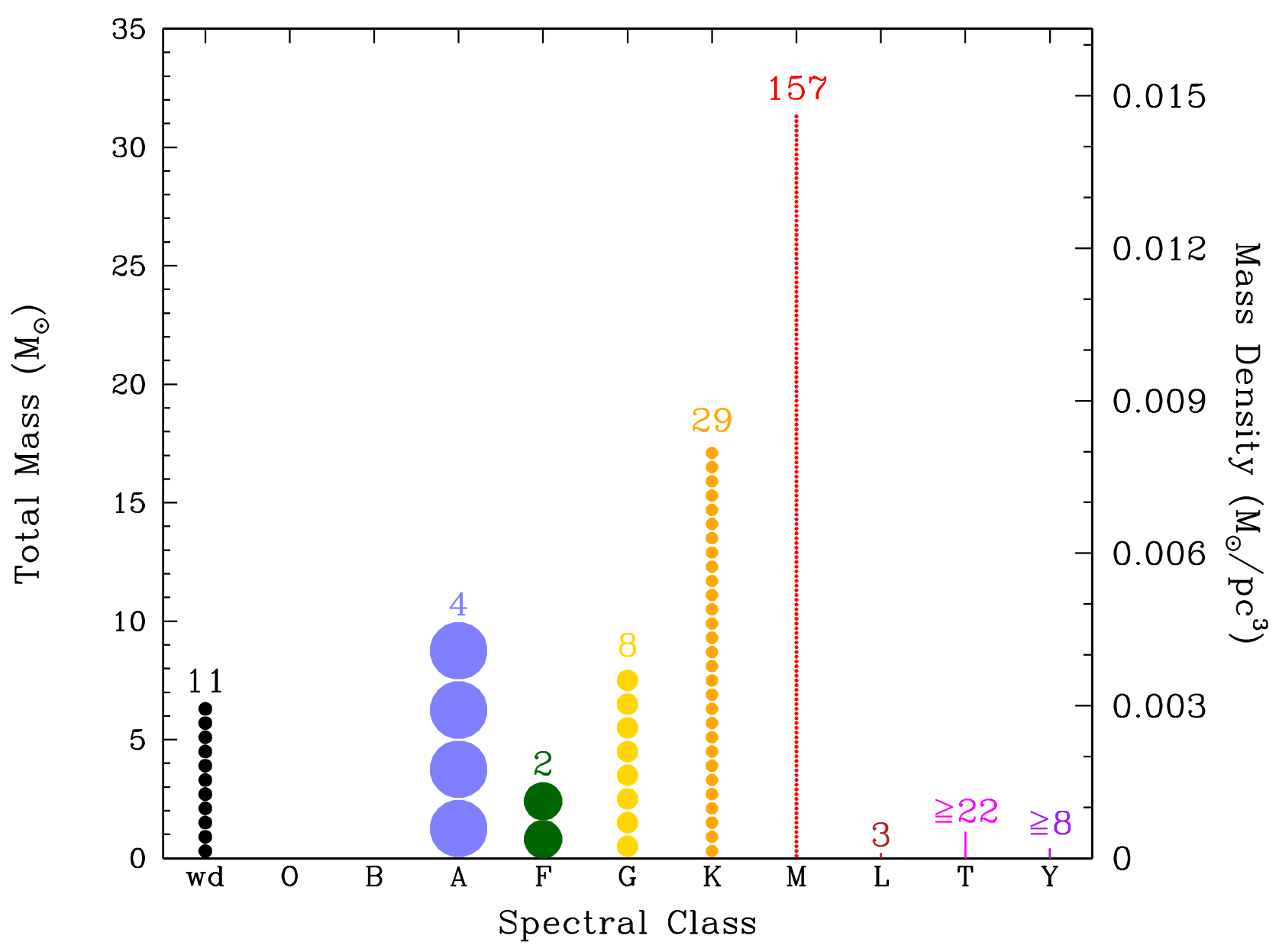

Fig. 11. - The 8pc sample as a function of spectral type plotted in three ways - as total mass (left axis), mass density (right axis), and histogram (numbers above each star stack). White dwarfs are shown in black, A stars in light blue, F stars in green, G stars in yellow, K stars in orange, $\mathrm{M}$ dwarfs in red, $\mathrm{L}$ dwarfs in firebrick, $\mathrm{T}$ dwarfs in magenta, and $\mathrm{Y}$ dwarfs in purple. The only bins believed to suffer from significant incompleteness are those of the $\mathrm{T}$ and $\mathrm{Y}$ dwarfs; it is likely that a small number of solivagant $\mathrm{T}$ dwarfs, and a larger number of $\mathrm{Y}$ dwarfs, have yet to be identified along with $\mathrm{T}$ and $\mathrm{Y}$ companions to higher-mass objects already known within the 8 pc volume. 


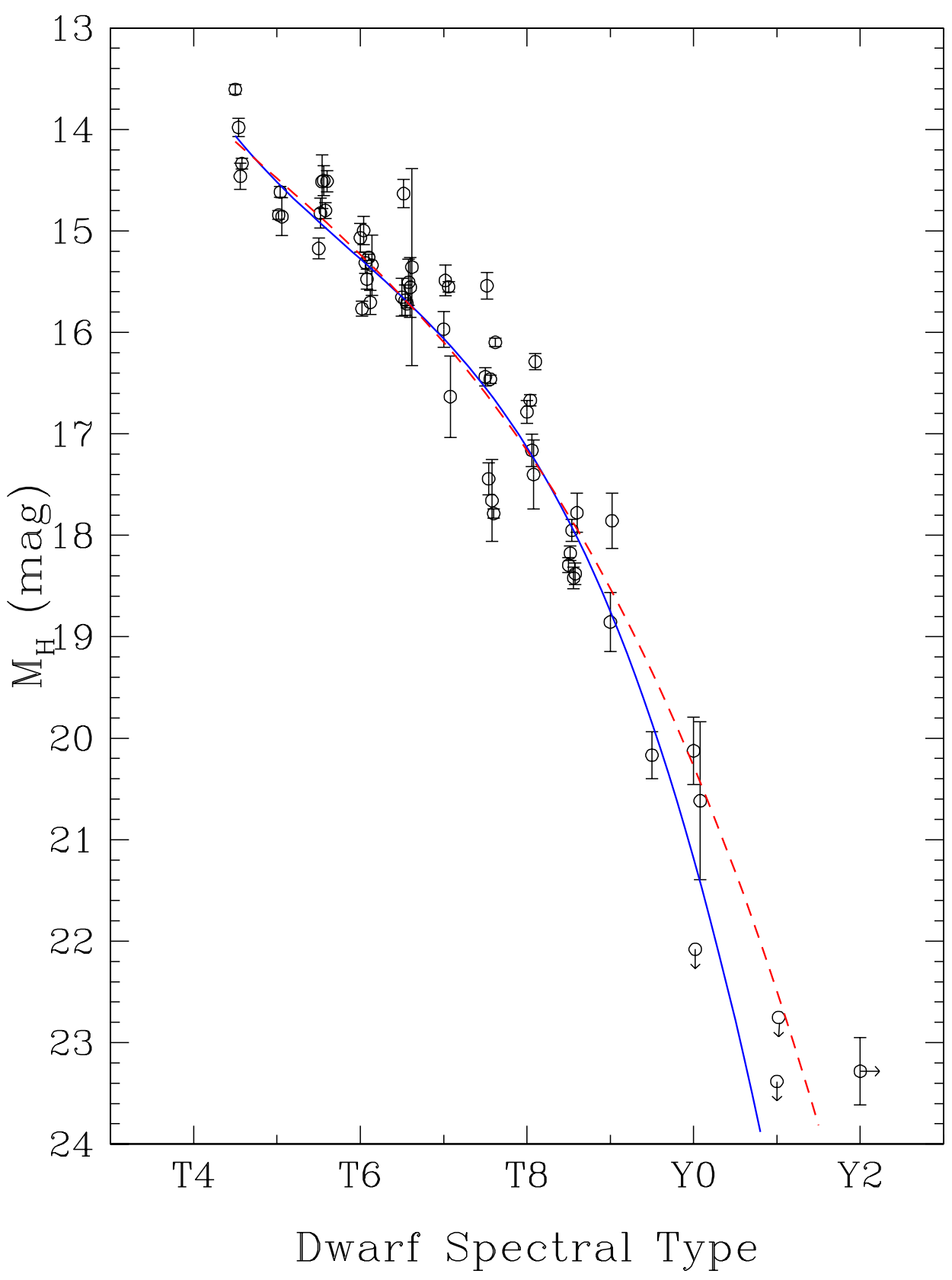

Fig. 12. - A plot of the absolute $H$-band magnitude of dwarfs with spectral types $>$ T4 and measured trigonometric parallaxes, from Table 6. Third-order fits to the data are shown by the dashed red curve (which includes the point for WISE 1828+2650 at lower right) and the solid blue curve (which excludes WISE 1828+2650). See Section 4.3 for details. 


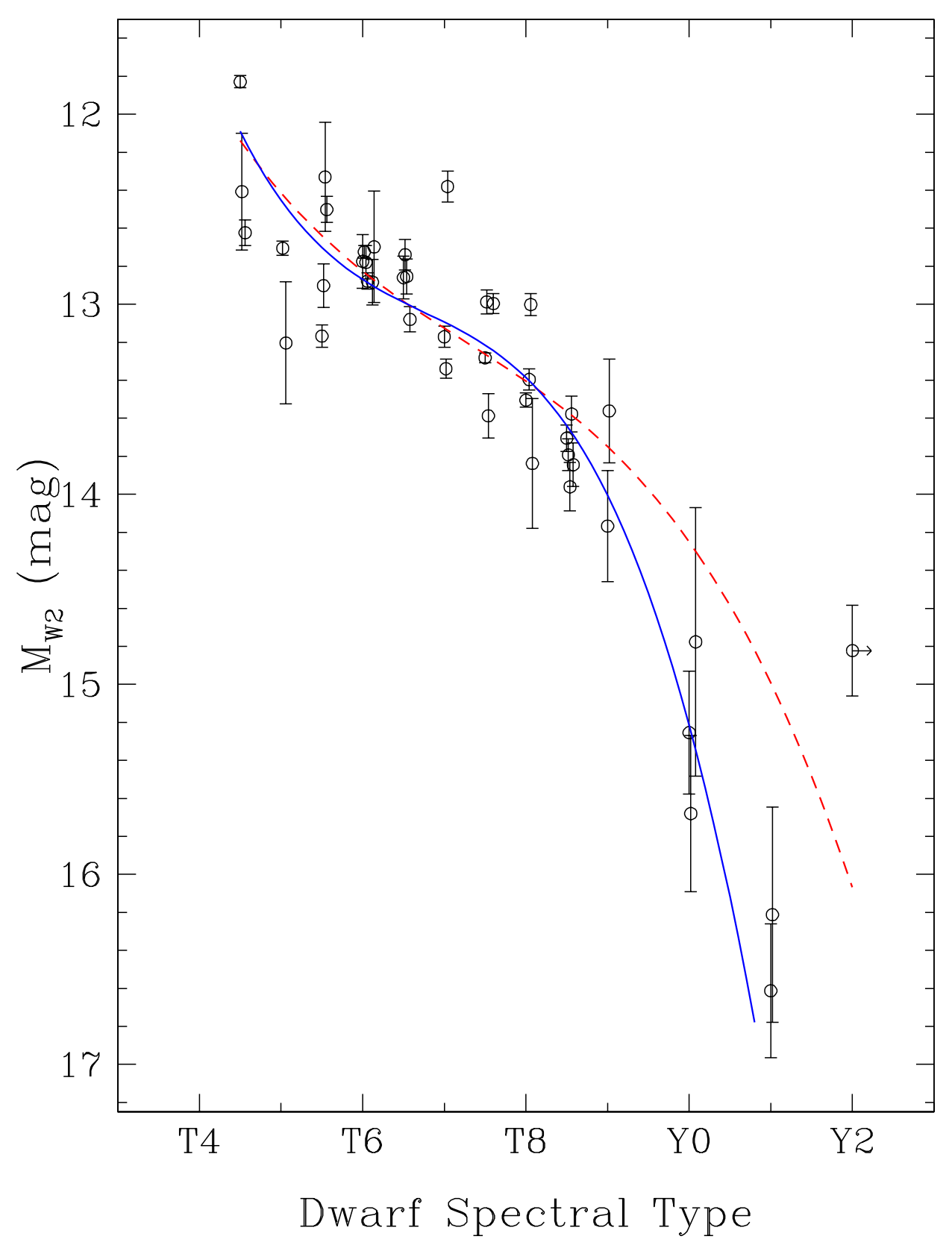

Fig. 13. - A plot of the absolute W2-band magnitude of dwarfs with spectral types $>$ T4 and measured trigonometric parallaxes, from Table 6. Third-order fits to the data are shown by the dashed red curve (which includes the point for WISE 1828+2650 near the right edge) and the solid blue curve (which excludes WISE 1828+2650). See Section 4.3 for details. 


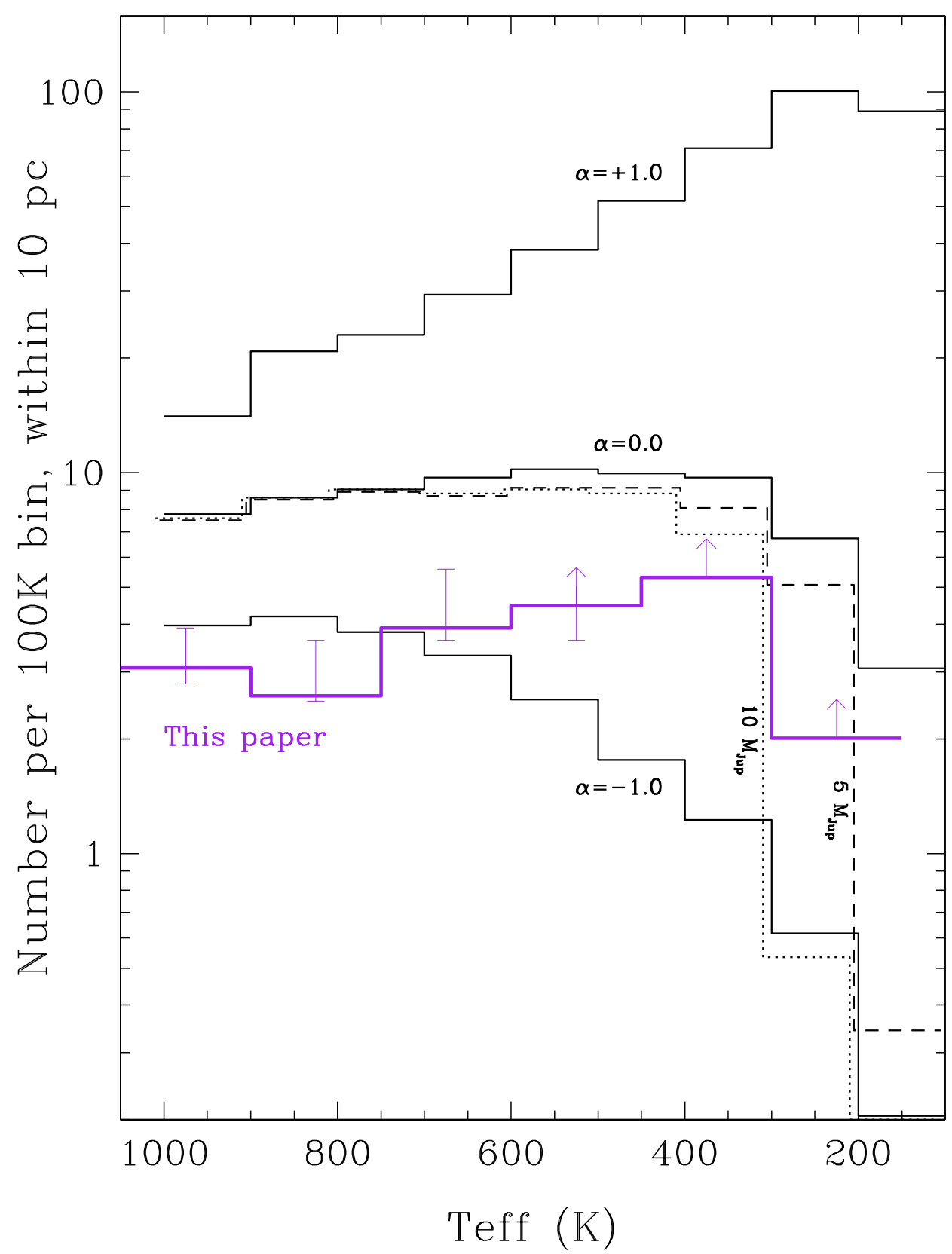

Fig. 14. - The predicted number of brown dwarfs within $10 \mathrm{pc}$ for three different power-law mass functions $\left(d N / d M \propto M^{-\alpha}\right)$ with $\alpha=-1,0$, and +1 (solid black) having a minimum formation mass of $1 M_{J u p}$. Also shown for the $\alpha=0$ model is the predicted number of brown dwarfs if a minimum formation mass of $5 M_{\text {Jup }}$ (dashed black) or $10 M_{\text {Jup }}$ (dotted black) is assumed. These simulations are from Burgasser et al. (2004, 2007). Space densities using our full accounting of objects in the immediate Solar Neighborhood (Tables 8 and 9) are shown by the heavy purple line. 


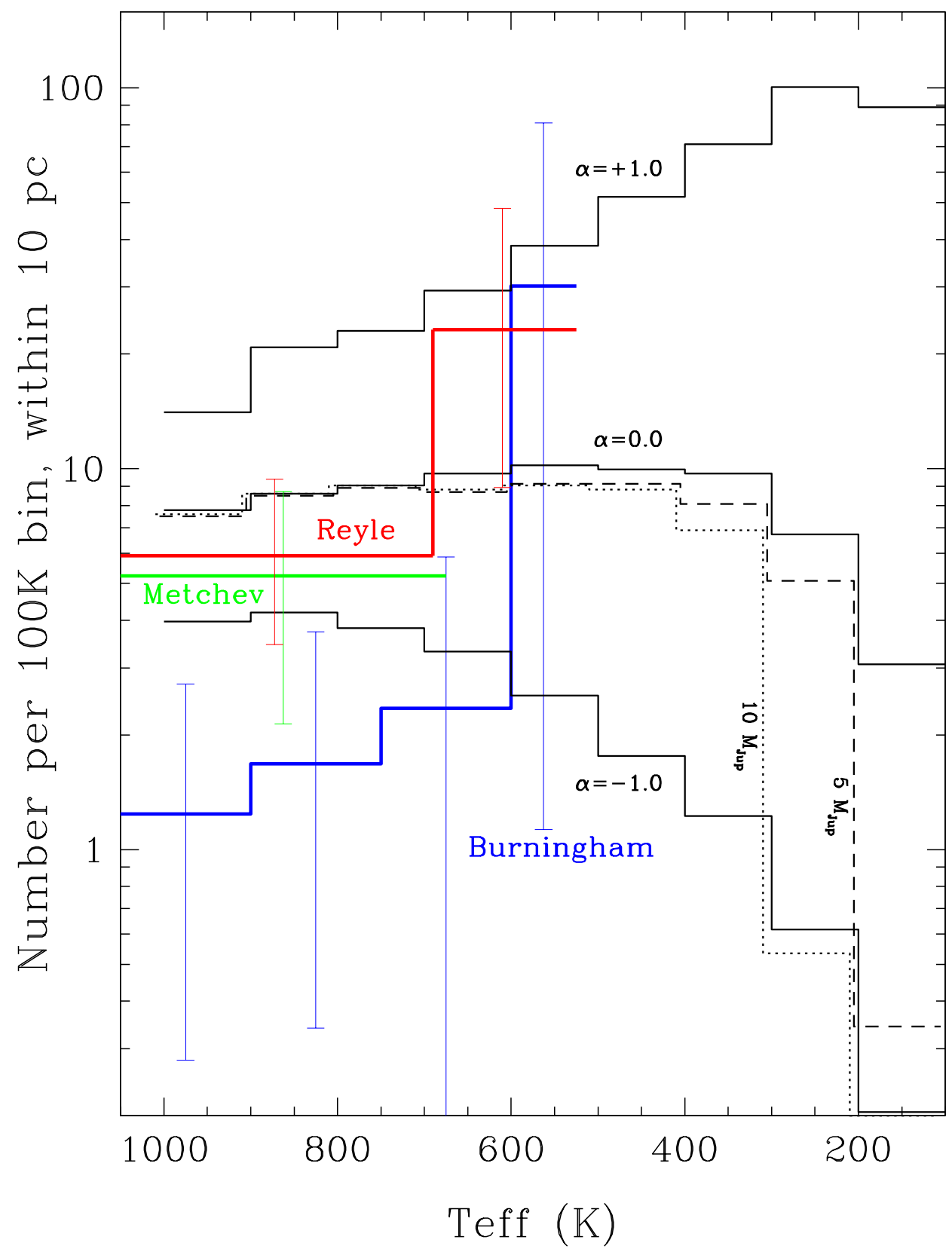

Fig. 15.- Previous measurements of the space density of mid- to late-T dwarfs from Metchev et al. (2008) (green), Reylé et al. (2010) (red), and Burningham et al. (2010) (blue) overplotted on the same simulations from Figure 14. 